\title{
Spectropolarimetry of Sunspot Penumbrae
}

\author{
Dissertation \\ zur Erlangung des Doktorgrades \\ der Mathematisch-Naturwissenschaftlichen Fakultäten \\ der Georg-August-Universität zu Göttingen
}

vorgelegt von
Nazaret Bello González
aus Granadilla de Abona / Santa Cruz de Tenerife / Spanien

Göttingen 2006 


\section{Bibliografische Information Der Deutschen Bibliothek}

Die Deutsche Bibliothek verzeichnet diese Publikation in der Deutschen Nationalbibliografie; detaillierte bibliografische Daten sind im Internet über http: / / dnb. ddb. de abrufbar.

D7

Referent: Prof. Dr. F. Kneer

Korreferent: Prof. Dr. M. Schüssler

Tag der mündlichen Prüfung: 15.06.2006

Copyright (C) Copernicus GmbH 2006

ISBN

Copernicus GmbH, Katlenburg-Lindau

Druck: Schaltungsdienst Lange, Berlin

Printed in Germany 
Dedicated to my parents 



\section{Contents}

$\begin{array}{ll}\text { Summary } & 7\end{array}$

1 Introduction 9

1.1 Sunspots . . . . . . . . . . . . . . . . . . . . 9

1.2 Sunspots: General properties . . . . . . . . . . . . . . . . 10

1.3 Penumbral fine-structure $\ldots \ldots \ldots \ldots \ldots$

1.4 Penumbral dvnamics: The Evershed effect . . . . . . . . . . . . . . . 12

1.5 The penumbral magnetic fine structure . . . . . . . . . . . . . . . . 13

1.6 Penumbral atmospheric models . . . . . . . . . . . . . . . . . . . . 14

1.7 Aim and outline of this work . . . . . . . . . . . . . . 15

\begin{tabular}{|lll}
2 & Introduction to spectropolarimetry & 17
\end{tabular}

2.1 Zeeman effect . . . . . . . . . . . . . . . . . . . . . 17

2.2 Stokes parameters . . . . . . . . . . . . . . . . . . . . . 21

2.2 .1 Measurements of Stokes parameters . . . . . . . . . . . 21

2.3 The radiative transfer equation . . . . . . . . . . . . . . . . . 23

2.3 .1 The propagation matrix . . . . . . . . . . . . . . . . . . 24

2.3 .2 Emission processes . . . . . . . . . . . . . . . . . 27

2.3 .3 The RTE for a magnetised atmosphere . . . . . . . . . . . 28

2.4 Solutions of the RTE and magnetic field measurements . . . . . . . . . 29

2.4 .1 Integration of the RTE: the DELO method . . . . . . . . . . . 29

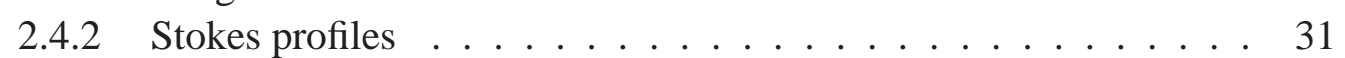

2.4.3 Stokes $I$ and $V$ profiles with varying magnetic field strength . . . 31

2.4 .4 Weak field approximation . . . . . . . . . . . . . 33

2.4 .5 Strong field regime . . . . . . . . . . . . . . . . . . . 34

2.4 .6 Centre of gravity method . . . . . . . . . . . . . . . . 35

3 Observations and data reduction 39

3.1 'Göttingen' FPI data . . . . . . . . . . . . . . . . . . . . . . . . 39

3.1.1 Description of the observations . . . . . . . . . . . 42

3.2 Data reduction and image restoration . . . . . . . . . . . . . 43

3.2 .1 Broad-band channel . . . . . . . . . . . . . . . . . . . 44

3.2 .2 Narrow-band channel . . . . . . . . . . . . . . . . . . . . . 47

3.2 .3 Deconvolution with a PSE $\ldots \ldots \ldots \ldots$

3.2 .4 Instrumental polarisation . . . . . . . . . . . . . . 50

3.2 .5 Scattered light . . . . . . . . . . . . . . . . . 52 
4 Penumbral fine-structure: data analvsis $\quad 53$

4.1 Intensity maps . . . . . . . . . . . . . . . . . . . . . 53

4.1 .1 Photometric properties of penumbrae . . . . . . . . . . . 55

4.1 .2 Spectroscopic properties of penumbral intensity . . . . . . . . . 59

4.1 .3 Temporal evolution . . . . . . . . . . . . . . . . . . 60

4.1 .4 Intensity fluctuations . . . . . . . . . . . . . . . . . 61

4.2 Magnetograms . . . . . . . . . . . . . . . 66

4.2 .1 Magnetic field maps from Fe I 6302.5 . . . . . . . . . . . . . 68

4.3 Velocitv maps . . . . . . . . . . . . . . . . . . . . . 70

4.3 .1 Bisectors and line minimum . . . . . . . . . . . . . 70

4.3 .2 Stokes $V$ zero crossing . . . . . . . . . . . . . . . . . . 72

4.3 .3 Centre of gravitv method . . . . . . . . . . . . . . . . 73

4.4 Comparison of intensitv. magnetic field and velocity maps . . . . . . 75

$4.4 .1 \quad$ Fe II 6149.3 observations at $\theta=23^{\circ} \ldots \ldots \ldots \ldots \ldots \ldots$. . . . . . 76

4.4.2 Fe I 6302 line pair observations at $\theta=20^{\circ} \ldots \ldots \ldots$. . . . 79

4.5 Intensity. LOS velocity and LOS magnetic field correlations . . . . . . 81

4.5 .1 Intensity vs. velocitv field . . . . . . . . . . . . . . . . . 82

4.5 .2 Intensitv vs. magnetic field . . . . . . . . . . . . . . 83

4.5 .3 Magnetic field vs. velocity field . . . . . . . . . . . . . . 85

5 Numerical modelling $\quad 89$

5.1 Motivation . . . . . . . . . . . . . . . . . . . . . . . . . 89

5.1 .1 Asvmmetries in the observed Stokes profiles . . . . . . . . 89

5.2 Two-component penumbral model . . . . . . . . . . . . . . 95

5.2 .1 Geometrv of the model . . . . . . . . . . . . . . . . 95

5.2.2 Atmospheric parameters for the one-component model . . . . . . 96

5.3 Svnthesis of Stokes profiles . . . . . . . . . . . . . . . . . . 101

5.3 .1 Reproduction of the observed asvmmetries . . . . . . . . . 101

5.3.2 Summary on the synthesis of Stokes profiles . . . . . . . . . . 107

6 Conclusions and outlook 109

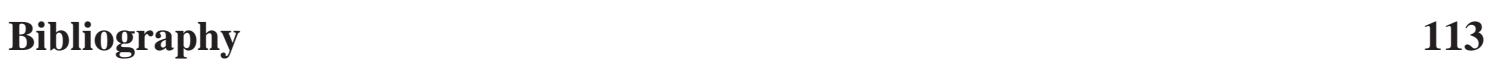

$\begin{array}{ll}\text { Acknowledgements } & 119\end{array}$

$\begin{array}{ll}\text { Publications } & 121\end{array}$

\begin{tabular}{ll}
\hline Lebenslauf & 123
\end{tabular} 


\section{Summary}

"No es perfecta, mas se acerca a lo que yo... simplemente soñé"

(El Breve Espacio) Pablo Milanés

Sunspots, the most prominent manifestation of the solar magnetism, are fascinating research subjects. Especially sunspot penumbrae, with their high structuring, have attracted much attention from which ample observational and theoretical scientific work has originated. Recent observations with improved solar instrumentation have revealed that sunspot penumbrae possess much finer structures than was thought a few decades ago. Thus, we are still at the beginning of understanding penumbrae with their still enigmatic intensity pattern, their magnetic field structures, and the basic processes which cause the structuring and the Evershed flow (Evershed 1909).

This Thesis work is based on two-dimensional high-spatial-resolution spectropolarimetric observations of sunspot penumbrae. They were obtained in April 2002 and May 2003 with the "Göttingen" Fabry-Pérot spectrometer at the Vacuum Tower Telescope at the Observatorio del Teide (Tenerife). Speckle methods were applied for image reconstruction which resulted in a spatial resolution of 0.5 in the magnetograms of the penumbrae. Stokes $I$ and $V$ profiles of the Fe II $6149 \AA$ line, which exhibits no instrumental Stokes $Q / U \rightarrow V$ crosstalk, and of the Fe I line pair at $6302 \AA$, have been analysed. From the analysis of intensity maps corresponding to different atmospheric layers it has been found that, on scales larger than 0.5 , the intensity pattern of penumbrae stays the same in the continuum and core images of the $6301.5 \AA$ line, which stem from $0 \mathrm{~km}$ and $300 \mathrm{~km}$ (above $\tau_{5}=1$ ), respectively. Yet at scales of 0.5 and smaller the pattern in the two spectral features it is found to be different.

From the comparison of velocity and intensity maps, one can see that in the limb side of sunspots the Evershed flow is carried by dark filaments and on the centre side by bright features and their somewhat weakened tails. This can be explain in terms of the picture in which the velocity of hot rising gas is best seen on the centre side, while on the limb side the horizontal outward and possibly downward flows are seen when the gas has cooled down.

Strong line-of-sight components of the magnetic fields are not found in bright filaments but in dark structures, somewhat displaced from the darkest parts. Their positions do not coincide with those of the strongest velocity fields. In general, these results are compatible with the picture of low lying flow channels coincident with the horizontal magnetic field, or possibly emerging and diving down into sub-photospheric layers, like a "sea serpent". 
To complete the analysis on the penumbral fine-structure, forward modelling has been performed. The aim has been to synthesise Stokes profiles which account for the asymmetries of the observed profiles. For that purpose, a numerical code was developed from existing ones to solve the one-dimensional radiative transfer problem. The assumed penumbral model contains two atmospheric components coexisting in the same resolution element. It has been possible to rule out "cool upflows" as an appropriate scenario of the penumbral structure. Moreover, synthetic profiles generated from the assumption of hot upflows have lead to the simulation of observed profiles from prenumbral grains. This would mean that the grains do indeed carry hot material to the photosphere in upflows. However, strongly asymmetric profiles are not easily reproducible with the considered model.

Some further dynamic phenomena are discussed along these pages, which demonstrate the richness of processes in penumbrae, and reveal unexpected properties. 


\section{Introduction}

\subsection{Sunspots}

Sunspots are known since ancient times (Wittman \& Xu 1987). They are observable with naked eye e.g. during the dawn and the sunset or through a hazy atmosphere. They appear as dark spots on the solar surface. It was not until the use of the first telescopes by Galileo Galilei and others in the early 17th century that it was discovered that sunspots indeed represented solar structures and that the Sun rotates. In sunspots a dark umbra and a less dark halo, the penumbra, could be distinguished. More than one century after, in 1769, Alexander Wilson observed on sunspots near the solar limb that the side of the sunspot closer to the disc centre decreased in size until disappearing while the sunspot was approaching the limb. Nowadays it is known that the so-called Wilson depression is due to decreased densities caused by the magnetic pressure and to the lower opacities in the sunspot photosphere which makes it appearing deeper with respect to the quiet-Sun photosphere by some 400-800 km (Gokhale \& Zwaan 1972).

During the 19th century works by Schwabe, Carrington and Wolf, among others, demonstrated the existence of the solar cycle of about 11 years, during which the heliographic latitude of the appearance of sunspots decreases monotonically, while the number of sunspots increases reaching its maximum at the middle of the cycle before decreasing until the minimum of activity at the end of the cycle, when the solar disc can be observed without any single sunspot. Sunspots are mainly restricted to the activity belts extended to heliographic latitudes of about $40^{\circ}$ on each side of the equator. This behaviour can be explained by models in which active regions are the emergence of large magnetic flux tubes through the solar surface where they are split into many smaller tubes, the larger of which constitute sunspots (e.g. Zwaan 1978), the most prominent example of solar flux tubes on the Sun. Today it is widely accepted that the magnetic fields are generated in the solar interior by a dynamo mechanism driven by differential rotation and convection (Charbonneau, 2005).

The first step to know about the physical nature of sunspots was made by G. E. Hale who, in 1908, measured magnetic fields from spectroscopic observations of the Zeeman effect in spectral lines. Since then, magnetic fields are known to be the main cause of sunspots and the key for the development of physical models attempting to explain the structure of sunspots and their evolution. The main generally accepted assumption was that magnetic fields inhibit, at least partially, the convective transport of energy and therefore sunspots appear cool and dark (Biermann 1941). 


\subsection{Sunspots: General properties}

Sunspots, as seen in white light, show a central dark umbra which radiates about $20 \%$ of the energy flux of the quiet Sun, surrounded by the penumbra radiating $80 \%$ of the energy flux of the quiet Sun. In terms of temperature, the umbra has an effective temperature of $T_{\text {eff }} \approx 3900 \mathrm{~K}$ and the penumbra $T_{\text {eff }} \approx 5400 \mathrm{~K}$ (the effective temperature of the quite Sun photosphere is $5780 \mathrm{~K}$ ). The lower temperatures are interpreted to be caused by the inhibition of convective energy transport by the magnetic fields. Average magnetic field strengths in a sunspot at photospheric level are found to vary from umbral values of 1800-3700 Gauss (Livingston 2002) to 700-1000 Gauss at the outer penumbra. The strongest field associated with the darkest part of the umbra is also close to vertical while at the outer boundary of the sunspot is inclined by $70-80^{\circ}$. The magnetic field strength also decreases with height. Chromospheric observations show that the magnetic field extends beyond the sunspot limit, as outlined in white-light images, forming a "canopy" (Giovanelli \& Jones 1982). The base of this canopy is almost horizontal and is located in high photospheric layers. It has been detected in observations with photospheric spectral lines (Solanki et al. 1992, Lites et al. 1993). Observations also suggest that in the upper chromospheric to lower coronal layers, the maximum field strength at the centre of regular sunspots still has values of 1000-1800 Gauss. Moreover, sunspots appear to be the photospheric counterpart of the footpoints of magnetic coronal loops which, when disturbed and interacting with other coronal loops, generate flares and coronal mass ejections. These observed properties of sunspots support the theoretical concept that sunspots as seen in white light are the intersections of the solar surface with large magnetic flux tubes emerging from the solar interior into the atmosphere as a manifestation of the solar dynamo. The structure of sunspots beneath the photosphere is still unclear in detail, mainly because of our lack in understanding the relevant magneto-convective processes (see the reviews of Weiss 1997, Weiss 2002, Jahn 1997, and also the recent work by Schüssler \& Rempel, 2005). The sub-photospheric structure is accessible observationally by local (time-distance) helioseismology (e.g., Lindsey \& Braun 1998).

The size of sunspots varies from $3500 \mathrm{~km}$ of diameter, for the smallest sunspots (Bray \& Loughhead 1964), to $60000 \mathrm{~km}$ for large sunspots. About lifetimes, the time scale of formation of a sunspot is from a few hours to several days, and they can live from some hours to months.

We refer to reviews by Wiehr (1999), Schlichenmaier (2003), Solanki (2003) and Bellot Rubio (2004) which contain many relevant references.

\subsection{Penumbral fine-structure}

Penumbrae, as seen in continuum images, are formed by a radial filamentary structure of alternating bright and dark fibrils with the bright fibrils showing point-like heads or 
penumbral grains (see Fig.1.11. In any case, 'dark' and 'bright' only has local significance, since bright features in one part of a penumbra may be darker than the dark structures in another part. Scharmer et al. (2002) and Rouppe van der Voort et al. (2004) have presented finest broad-band observations from the new Swedish $1 \mathrm{~m}$ solar telescope in La Palma. After image reconstruction, penumbral filaments at the diffraction limit of 0'.1 show the newly detected "dark cores". Sütterlin (2001) concludes from G-band speckle-reconstructed images that $250 \mathrm{~km}$ should be a typical size for the penumbral fine structure. Scharmer et al. (2002) measure a width of the penumbral filaments of 150$180 \mathrm{~km}$ claiming that the penumbral filaments have thus been resolved.

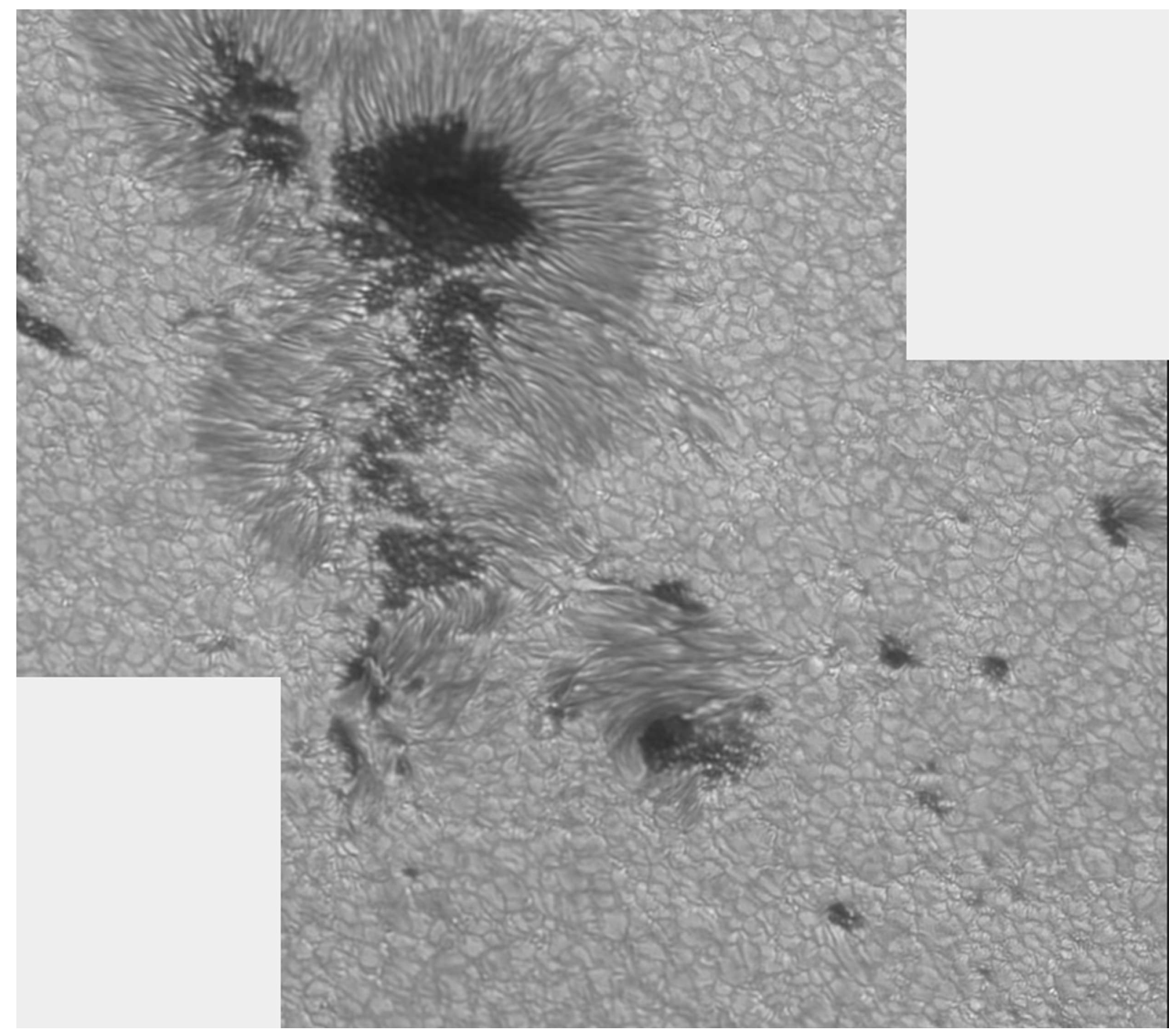

Figure 1.1: Broad-band image of sunspots NOAA 0875 at heliocentric angle $42^{\circ}$. Observation with the Vacuum Tower Telescope at Observatorio del Teide (Tenerife) on April 26, 2006, image size $94^{\prime \prime} \times 83^{\prime \prime}$ (courtesy of B. Sánchez-Andrade Nuño, K. G. Puschmann \& J. Blanco Rodríguez).

Penumbral filaments live from $10 \mathrm{~min}$ to 4 hours, and penumbral grains from $20 \mathrm{~min}$ to one hour. Following the temporal evolution of penumbrae, one can observe that the penumbral grains move radially inward in the inner penumbra, while outward motions of structures are seen in the outer penumbra (Denker 1998, Sobotka \& Sütterlin 2001). 
The observed inward motions of intensity structures in the inner penumbra must not be associated with mass motions since flow velocities are always directed outward in the penumbra, as will be described below.

Small-scale structures are also found at chromospheric heights, although larger scales tend to dominate. These structures often appear in the super-penumbra visible in $\mathrm{H} \alpha$ line core observations. The fibrils seen in $\mathrm{H} \alpha$ are associated with inflow material, the inverse Evershed effect (see below, Moore \& Rabin 1985, Bray \& Loughhead 1964).

\subsection{Penumbral dynamics: The Evershed effect}

The strong dynamics shown by sunspot penumbrae at photospheric level is known as the Evershed effect (Evershed 1909). It is characterised by a blueshift of photospheric spectral lines in the centre side penumbra (part of the penumbra closer to the solar disc centre) and a corresponding redshift in the limb side penumbra (part of the penumbra farer from the solar disc centre). Often, the shifts in wavelength are accompanied by line asymmetries. These line shifts and asymmetries in the lines increase from the inner to the outer penumbra. The Evershed effect is dependent on height in the penumbral atmosphere. In the photosphere the line shifts decrease rapidly with height (Börner \& Kneer 1992, Rouppe van der Voort 2002). Above the temperature minimum, the line shifts change sign, i.e. redshits are observed in the centre-side penumbra and blueshifts in the limb-side penumbra. This is the so-called inverse Evershed effect. Also the Evershed effect is better seen in sunspots off disc centre. Thus, the Evershed effect is interpreted as a radial outflow of material from the inner penumbra to the outer penumbra at photospheric level, and an inflow with larger velocity in the chromosphere. Yet the mass flux associated with the inverse Evershed effect is only a few percent of the photospheric Evershed flow, due to the lower gas density in the chromosphere.

Attempts to find a correlation of the Evershed effect with the penumbral structures at small scales has lead to controversial results. Some authors find that the Evershed flows correlate with dark filaments (e.g. Schröter 1965, Beckers 1968, Stellmacher \& Wiehr 1971, Wiehr et al. 1984, Title et al. 1993, Rimmele 1995, Rouppe van der Voort 2002). Other authors do not find a significant spatial correlation (Lites et al. 1990, Hirzberger \& Kneer 2001). Degenhardt \& Wiehr (1994) and Rimmele (1995) find a good anti-correlation between velocity and brightness when they compare these magnitudes at similar atmospheric levels. Similarly, no obvious correlation between brightness and magnetic field strength and field inclination can be established.

When the Evershed effect is considered to be caused by the flow of material, one possible explanation of the driving mechanism is given by the siphon flow models originally proposed by Meyer \& Schmidt (1968) and developed by Montesinos \& Thomas (1993, 1997). The siphon flow assumes that the mass flow is driven in flux tubes along the magnetic field lines, by the difference in gas pressure of their footpoints. Such pressure imbalance is caused by the difference in magnetic field strength at the footpoints at 
the same geometric height. Thus, if one of the footpoints is located in the penumbra with some field strength of 1000 Gauss and the second in a small magnetic element outside the sunspot with concentrated magnetic flux with field strength of 1100-1500 Gauss, the material would be driven by the higher pressure in the penumbra outwards to the external magnetic element. In the case that the footpoint were located not in the penumbra but in the umbra with a magnetic field strength of 2500 Gauss, then the material would be driven inwards from the external magnetic flux concentration (inverse Evershed flow). This model can explain the wavelength shifts of the spectral lines but it has some difficulties to explain the observed asymmetries. In addition, it is not yet established from observations that, at the same geometrical height, the field strength in the inner penumbra is lower than in the outside magnetic flux elements.

Studying the dynamical properties of thin flux tubes within the penumbra, Schlichenmaier et al. (1998) presented numerical simulations of the evolution of a thin flux tube embedded in a two-dimensional background model. The tube is initially lying along the sub-atmospheric magnetopause, which separates the penumbra from the non-magnetic quiet Sun. The tube is radiatively heated by the underlying hotter quiet Sun, it becomes buoyant, and due to the high inclination of the magnetopause with respect to the vertical, the buoyancy forces overcome the stabilising magnetic forces and the tube starts to rise from sub-photospheric layers. In the photosphere, the flow bends into horizontal direction and continues radially outwards. Since the radiative heat exchange is very efficient in the photosphere, the plasma flow cools down. The effect of this cooling means a reduction of the gas pressure in the tube and hence a gas pressure gradient along the tube which accelerates the plasma outwards (the syphon mechanism). During the emergence of the tube through the sub-photospheric penumbra, the footpoint of the tube migrates towards the umbra reproducing the observed inward motions of the penumbral grains. Schlichenmaier (2003) has presented new simulation results in which the tube is considered to be not horizontal but a wave along the photospheric part of the tube, resembling a "sea serpent" which merges and submerges in the photosphere, as represented in a snapshot in Fig.1.2 Thus, the model does also account for the observed outward motion of the penumbral structures in the outer penumbra.

\subsection{The penumbral magnetic fine structure}

Penumbrae show also a filamentary structure when seen in polarised light, from visible and infrared lines (see Bellot Rubio 2004). With increasing resolution such filamentary structure becomes narrower, indicating that magnetic fine structure has not yet been resolved. To characterise the sunspot magnetism, many authors study the net circular polarisation, $\mathrm{NCP} \equiv \int V(\lambda) \mathrm{d} \lambda$, which accounts for the degree of asymmetry of the circularpolarisation profiles. Sánchez Almeida \& Lites (1992) found that the amount of NCP of visible lines emerging from the penumbra is so large, that gradients in velocity as well as gradients in magnetic field inclination have to be present in the penumbral structure to account for it. 

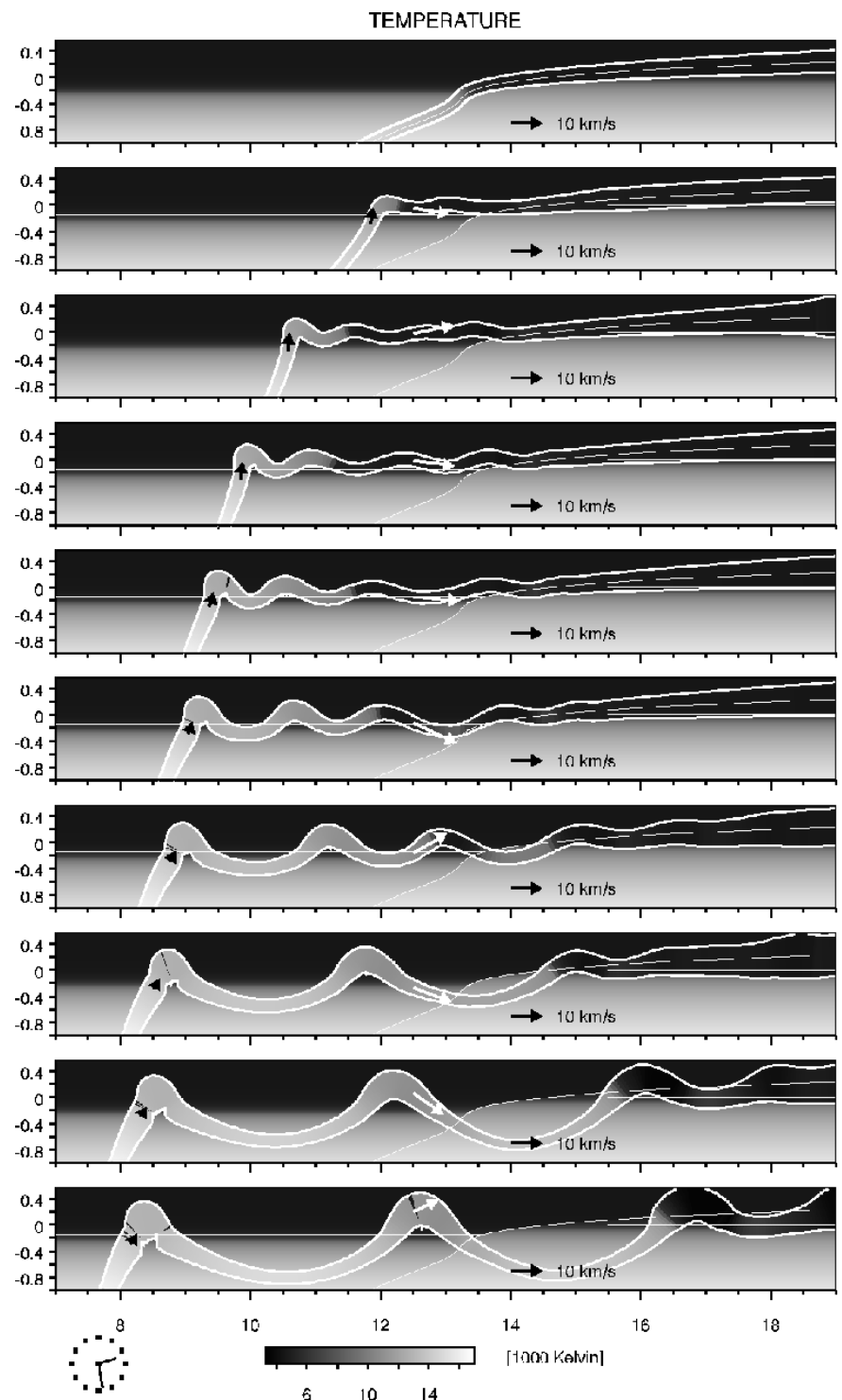

Figure 1.2: The evolution of a thin magnetic flux tube. Snapshots of the tube, with its diameter magnified by a factor of 4, are shown every $1000 \mathrm{~s}$. Initially, the tube lies along the magnetopause, is heated by the hotter quiet Sun, and rises through the penumbra such that its footpoint migrates inwards. The gray coding represents temperature. The black and white arrows correspond to the flow velocity along the tube. The $\tau=1$ level of the quiet Sun and the penumbra with a Wilson depression of $150 \mathrm{~km}$ are marked with horizontal lines. From Schlichenmaier (2003).

\subsection{Penumbral atmospheric models}

Several penumbral models have been proposed and developed aiming to account for the penumbral fine-structure. Del Toro Iniesta et al. (1994) have inverted 801 profiles of the Fe I $5576 \AA$ line to derive the temperature stratification at each spatial location. They have created a model of the average penumbral temperature stratification by combining the temperatures of 411 of their models. Rouppe van der Voort (2002) constructed three 
models to represent cool, hot and intermediate structures in the penumbra. He discusses the difference in stratification between the disc- and limb-side penumbra, with the former being slightly hotter.

Solanki \& Montavon (1993) proposed the uncombed penumbral model, i.e. a three layered model consisting of horizontal flow channels (central layer) embedded in an inclined magnetic field (lower and upper layers). Forward modelling has been used to successfully reproduce the center-to-limb variation of the NCP in penumbrae (Solanki \& Montavon 1993, Martínez Pillet 2000), and to explain the radial dependence of the amplitude asymmetry of Stokes $V$ profiles (Schlichenmaier \& Collados 2002) and the azimuthal variation of NCP within the penumbra (Schlichenmaier et al. 2002, Müller et al. 2002).

Recently, Spruit \& Scharmer (2006) proposed a new model for the penumbral fine structure as an extension of models proposed for umbral dots. This model, the "gappy penumbra", possesses field-free convective, radial gaps in the sub-atmospheric layers with their cusps intruding into the visible layers. The model accounts for the energy supply needed to balance the radiative losses from penumbrae. It also aims at explaining the origin of the dark cores as the top of the cusps where the high opacity makes them to appear dark between the bright filaments, i.e. the hot walls of the convective layers. This model does not deal with dynamics of sunspot penumbrae.

\subsection{Aim and outline of this work}

After one century of investigations since the first observational evidences of their magnetic and dynamic nature were found, sunspot penumbrae remain still enigmatic. We still do not understand in detail the origin of their formation in pores and their stable structuring at small-scales where magnetic fields and mass flow seem to coexist. As pointed out by Bellot Rubio (2004), the penumbra can be understood as a collection of small flux tubes embedded in a magnetic background atmosphere and there is growing evidence that, even at high spatial resolution of current spectroscopic observations, the tubes are still unresolved horizontally.

Therefore, the key for the study of the penumbral dynamics and magnetic fields consists in spectropolarimetry at high spatial resolution. We need to obtain and to analyse the information carried by suitable spectral lines, we need to measure and interpret the polarised radiation from the penumbra. In this respect, this work aims at contributing to the understanding of the penumbra at small-scales from the analysis of spectropolarimetric observations with spatial resolution better than 0.5 .

In the following pages, the concepts and mathematical formulations commonly used in spectropolarimetry as well as an introduction to the radiative transfer in magnetised atmospheres are reviewed along Chapter 2. Chapter 3 is devoted to the description of two-dimensional spectropolarimetric observations, the data reduction and the reconstruc- 
tion of the two-dimensional filtergrams. The reconstruction corrects the images for the degradation caused by the terrestrial atmosphere leading to the achievement of the abovementioned high angular resolution. Next, in Chapter 4 the analysis of the data from the calculation of magnetograms and velocity maps from different methods and the results extracted from them are described. Chapter[5 contains the consequential step following the data analysis, the synthesis of spectral lines by solving the radiative transfer equation for a penumbral atmospheric model containing a background and embedded inhomogeneities. The final Chapter 6 concludes this Thesis and gives an outlook. 


\section{Introduction to spectropolarimetry}

\subsection{Zeeman effect}

P. Zeeman discovered in 1896 that, in the presence of a magnetic field, spectral lines are split into several polarised components. This Zeeman effect provides also information about the magnetic fields on the Sun. It was used by G.E. Hale in 1908 to detect strong magnetic fields in sunspot. 1

The origin of the phenomenon can be explained when analysing the "reaction" of atoms exposed to a magnetic field. For that purpose it is sufficient to consider the RussellSaunders or LS coupling approximation (which only takes into account the electrostatic interaction of the electrons). In this case, the quantum numbers $L, S, J, M$ define the state of an atom. $L$ is the quantum number for the total orbital angular momentum of the electrons, $S$ for the total spin angular momentum, $J$ for the total angular momentum $(\mathbf{J}=\mathbf{L}+\mathbf{S})$ and $M$ is the magnetic quantum number taking values of $-J,-J+1, \ldots, J-1, J$. The state of an atom is usually written as ${ }^{2 S+1} L_{J}$ and for given $L$ and $S$ there are $(2 L+1) \times(2 S+1)$ possible states.

In the absence of magnetic field, the energy of the state does not depend on $M$. In the presence of a weak magnetic field (weak in the sense that changes which it produces in the energy of the state are small compared to the energy difference of the levels), the increment of energy of the state is given by

$$
\delta E=\frac{e h B}{4 \pi m_{e} c} g M
$$

where $m_{e}$ and $e$ are the mass and charge of the electron, $h$ the Planck constant, $c$ the speed of light, $B$ the magnetic field strength and $g$ the Landé factor defined by

$$
g \equiv \frac{3}{2}+\frac{S(S+1)-L(L+1)}{2 J(J+1)} .
$$

When a transition occurs from a higher, or upper, energy level (from now on denoted by subindex $u$ ) to a lower energy level (denoted by subindex $l$ ), a photon is emitted, and a photon is absorbed when the transition goes from the lower energy level to the upper energy level. In both cases the energy of the emitted or absorbed photon is

\footnotetext{
${ }^{1}$ In the astrophysical literature, the Gauss system of units is widely in use. Besides, in the solar atmosphere, $H=B$, and throughout this thesis $B$ is measured in Gauss, where $10^{4}$ Gauss $=1 \mathrm{~T}$ (Tesla).
} 


$$
\Delta E=\Delta E_{0}-\frac{e h B}{4 \pi m_{e} c}\left(g_{l} M_{l}-g_{u} M_{u}\right)
$$

where $\Delta E_{0}$ is the energy difference between the upper and the lower level in the absence of magnetic field, and $g_{l}, g_{u}$ and $M_{l}, M_{u}$ are the Landé factors and the magnetic numbers of the lower and upper states, respectively. The wavelength shift of the photon with respect to the un-shifted $\lambda_{0}$ (not affected by $B$ ) is given by

$$
\lambda-\lambda_{0}=\frac{\lambda_{0}^{2} e B}{4 \pi m_{e} c^{2}}\left(g_{l} M_{l}-g_{u} M_{u}\right)=\lambda_{B}\left(g_{l} M_{l}-g_{u} M_{u}\right) .
$$

We denote $\lambda_{B}=\lambda_{0}^{2} e B / 4 \pi m_{e} c^{2}$ as the Zeeman splitting and inserting the values for the constants, with $\lambda_{B}$ and $\lambda$ in $\AA$ and $B$ in Gauss we have

$$
\lambda_{B}=4.67 \times 10^{-13} \lambda_{0}^{2} B .
$$

The selection rules for electric dipole transitions which apply for the "allowed" spectral lines are $\Delta L=0, \pm 1, \Delta S=0, \Delta J=0, \pm 1$ (except for $J_{u}=J_{l}=0$ ) and $\Delta M=0, \pm 1$. It is then possible to distinguish three components of a spectral line in the presence of a magnetic field according to the values of $\Delta M$ :

- Transitions with $\Delta M=0$ produce the $\pi$-components or linearly polarised components of the light.

- Transitions with $\Delta M=+1$, i.e. $M_{u}=M_{l}+1$, produce the $+\sigma$-components or circularly polarised components in the plane perpendicular to the direction of the linear polarisation of the $\pi$-components.

- Transitions with $\Delta M=-1$, i.e. $M_{u}=M_{l}-1$, produce the $-\sigma$-components or circularly polarised components opposite to the $+\sigma$-components.

Summarising, in the absence of magnetic field, all components described above are at the same wavelength $\left(\lambda_{0}\right)$, and if all the atomic levels $M$ are equally populated (as in Local Thermodynamical Equilibrium, LTE), the different states of polarisation are compensated and there is no polarisation. However, in the presence of a magnetic field, the degeneracy of the states with different quantum number $M$ is broken, and the $\pi$ and $\sigma$ components occur at different wavelengths, as depicted schematically in Fig.2.1 It is then possible to observe the states of polarisation of the various components.

Denoting the $+\sigma$-component by $\sigma_{b}$ (for blue) and the $-\sigma$-component by $\sigma_{r}$ (for red), the displacement of each component is given by:

$$
\begin{aligned}
\Delta \lambda_{\pi} & =M_{l}\left(g_{l}-g_{u}\right) \lambda_{B} \\
\Delta \lambda_{\sigma_{b}} & =\left[M_{l}\left(g_{l}-g_{u}\right)-g_{u}\right] \lambda_{B} \\
\Delta \lambda_{\sigma_{r}} & =\left[M_{l}\left(g_{l}-g_{u}\right)+g_{u}\right] \lambda_{B} .
\end{aligned}
$$

The Zeeman effect was discovered before the development of the quantum theory and, therefore, its origin was initially explained through classic electrodynamics which only could account for the Zeeman patterns with one central $\pi$-component and two displaced 


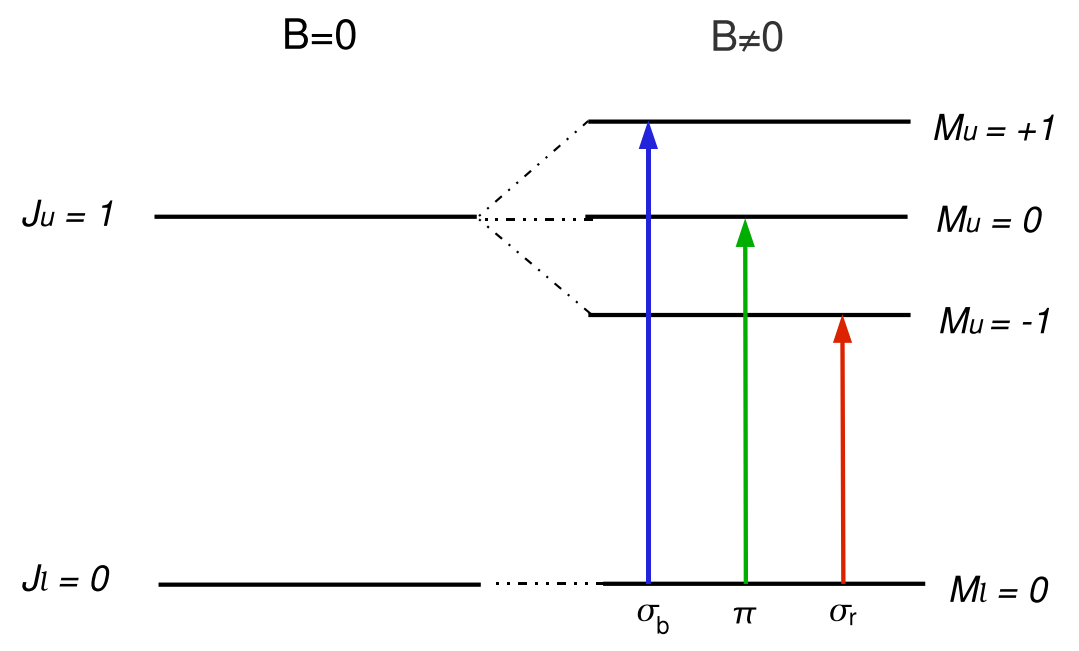

Figure 2.1: Schema of energy levels for a Zeeman triplet: one un-shifted $\pi$-component (green) and two shifted $\sigma$-components (blue and red).

$\sigma$-components, today still denoted by "normal" Zeeman effect. Occasionally, it is useful to approximate the pattern of a Zeeman multiplet by a "normal" Zeeman triplet. For that purpose, the $\sigma_{r}, \sigma_{b}$ and $\pi$ components are substituted by averages in intensity and displacements of the individual components. The average displacements are zero for the $\pi$-components due to the symmetry of the Zeeman pattern and for the $\pm \sigma$-components it follows

$$
\Delta \lambda_{\langle \pm \sigma\rangle}=\mp g_{\mathrm{eff}} \lambda_{B}
$$

or in absolute value and substituting $\lambda_{B}$,

$$
\Delta \lambda_{B}=4.67 \times 10^{-13} \lambda_{0}^{2} B g_{\text {eff }}
$$

where the effective Landé factor $g_{\mathrm{eff}}$ is introduced as

$$
g_{\mathrm{eff}}=\frac{1}{2}\left(g_{u}-g_{l}\right)+\frac{1}{4}\left(g_{u}-g_{l}\right)\left[J_{u}\left(J_{u}+1\right)-J_{l}\left(J_{l}+1\right)\right] .
$$

It accounts for the magnetic sensitivity of a spectral line: the higher the value of $g_{\text {eff }}$, the higher the response of the line to the magnetic field effects. This expression is also valid beyond the approximation of $L S$ coupling, but then empirical values for $g_{\text {eff }}$ are needed.

Longitudinal Zeeman effect. When the magnetic field $\boldsymbol{B}$ is parallel to the line of sight (LOS), the observer sees only the $\sigma$-components which are circularly polarised in both, the clockwise and counterclockwise sense. In the case of emission, the light travelling into the direction of $\boldsymbol{B}$ is left-circularly polarised (seen from the observer) for the blue $\sigma$-component and right-circularly polarised for the red $\sigma$-component, as sketched in the left part of Fig.2.2 In the case of absorption the polarisation states are reversed. The right part of Fig.2.2 depicts the Stokes $I$ and $V$ profiles for the case of absorption. By convention, positive $V$ means right-circularly polarised light (see Sec.2.2 for the Stokes parameters). 
Longitudinal Zeeman effect

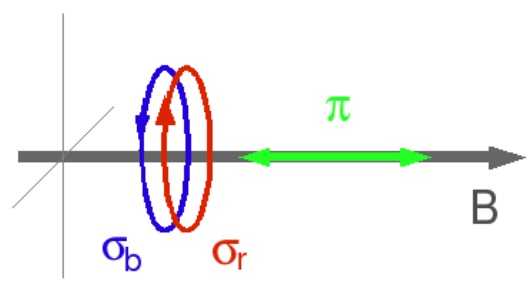

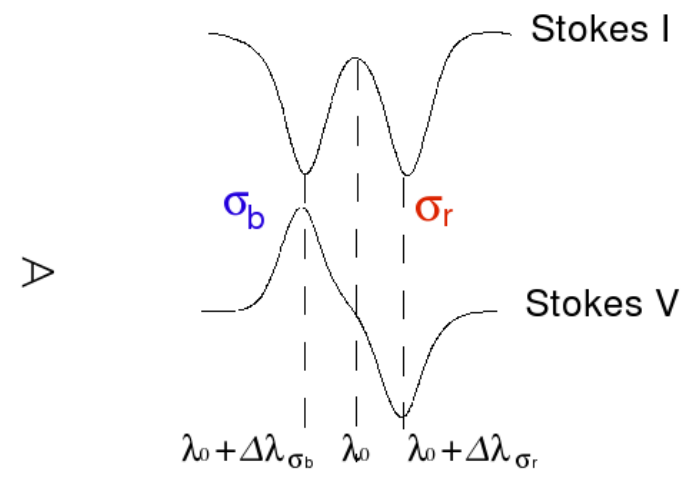

Figure 2.2: Schema of the longitudinal Zeeman effect.

Transverse Zeeman effect. In the case when $\boldsymbol{B}$ points in the direction perpendicular to the line of sight, the observer sees the $\pi$-components and the $\sigma$-components (Fig.2.3). In emission, the $\pi$-component(s) is (are) linearly polarised parallel to the direction of $\boldsymbol{B}$ and the $\sigma$-components show light linearly polarised perpendicular to $\boldsymbol{B}$, i.e. the projection of the circularly polarised light into the direction of the observer. In absorption, the oscillating electrons reduce, i.e. take out linearly polarised light. Thus, the (partial) polarisation is reversed compared to the case of emission as sketched on the right part of Fig. 2.3. Again by convention, positive $Q$ means horizontal linearly polarised light.
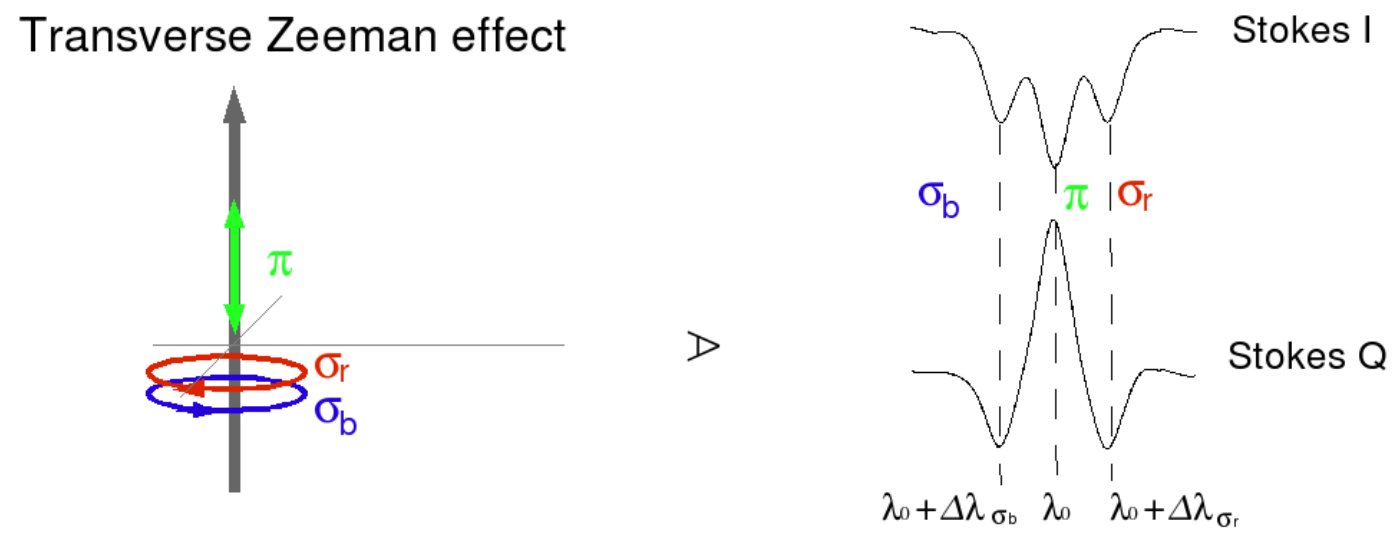

Figure 2.3: Schema of transverse Zeeman effect.

When, in the most general case, $\boldsymbol{B}$ is pointing in some arbitrary direction, the projection along the line of sight of the $\pi$ and $\sigma$ components will be seen. This effects will be treated again in the next section when introducing the Stokes parameters to understand the magnetic field information which can be inferred from the polarimetric observational data. 


\subsection{Stokes parameters}

Let us consider the representation of electromagnetic radiation in terms of a monochromatic plane wave propagating in the $z$ direction. The electric vector can be then characterised by

$$
\mathbf{E}_{x}=E_{x} \cos (\omega t+k z), \quad \mathbf{E}_{y}=E_{y} \cos (\omega t+k z+\phi), \quad \mathbf{E}_{z}=0,
$$

where $E_{x}$ and $E_{y}$ are the amplitudes, $\phi$ the difference of phase between the two components of the electric field. The Stokes parameters introduced by Sir George Stokes in 1852 , are defined by

$$
\begin{array}{ll}
I=E_{x}^{2}+E_{y}^{2}, & U=2 E_{x} E_{y} \cos \phi, \\
Q=E_{x}^{2}-E_{y}^{2}, \quad V=2 E_{x} E_{y} \sin \phi .
\end{array}
$$

In practice, natural light is never perfectly monochromatic. If we consider now a beam of small enough bandwidth it can be treated as a wave packet. In this case, the Stokes parameters are defined as averages of the superposition of various independent plane waves forming the wave packet. Hence, we can write

$$
\begin{array}{ll}
I=\left\langle E_{x}^{2}+E_{y}^{2}\right\rangle, & U=2\left\langle E_{x} E_{y} \cos \phi\right\rangle, \\
Q=\left\langle E_{x}^{2}-E_{y}^{2}\right\rangle, & V=2\left\langle E_{x} E_{y} \sin \phi\right\rangle .
\end{array}
$$

The Stokes vector $\mathbf{I}=(I, Q, U, V)^{T}$ characterises completely the polarisation state of the radiation:

- I represents the total intensity,

- $Q$ and $U$ describe the linearly polarised light of the beam and

- $V$ the circularly polarised radiation.

Therefore, when the light is unpolarised $Q=U=V=0$. At the other extreme, when the light is completely polarised we have $I^{2}=Q^{2}+U^{2}+V^{2}$ and, in the general case of partial polarisation, the degree of polarisation is given by

$$
P=\left(\frac{Q^{2}+U^{2}+V^{2}}{I^{2}}\right)^{1 / 2} .
$$

\subsubsection{Measurements of Stokes parameters}

The polarisation of the radiation field under study is caused by magnetic field in the regions from where the radiation originates. Therefore, measurements of polarised light through measurements of the Stokes parameters are the key to extract information about the orientation and strength of the magnetic fields housed in the magnetic solar structures under observation. 


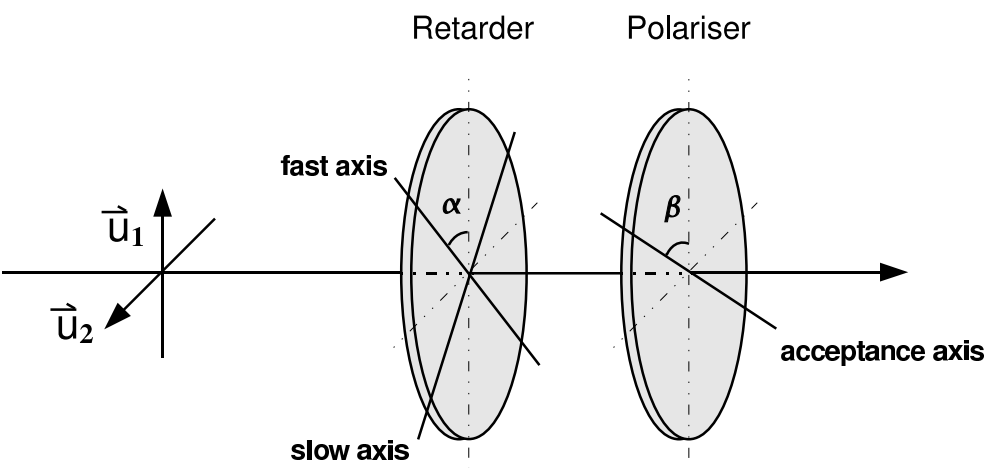

Figure 2.4: Scheme of a polarimeter composed by a retarder and a linear polariser. The $\mathbf{u}_{1}$ and $\mathbf{u}_{2}$ vectors define the plane of the electric field of the incident radiation. The retarder is characterised by a fast and a slow axis and the angle $\alpha$. The polariser is characterised by the acceptance axis and the angle $\beta$.

The Stokes vector can be measured in various manners. Following Landi degl'Innocenti (1992), we can consider a prototype polarimeter by means of two optical devices: a retarder and a polariser.

An ideal polariser is totally transparent to a radiation whose electric field vector vibrates along a given axis, the acceptance axis of the polariser, and opaque to electric fields vibrating in perpendicular direction to this axis. An ideal retarder is characterised by a fast and a slow axis and divides the incident ray in two linearly polarised components along the two axes, dephases one component with respect to the other and then reunites back the two components. Thus, the electric field of the two, fast and slow, components inside the retarder (along $z$ ) is described by

$$
E_{f}(z, t)=\varepsilon_{f} e^{\left(k_{f} z-\omega t\right)}, \quad E_{s}(z, t)=\varepsilon_{s} e^{\left(k_{s} z-\omega t\right)}
$$

where $k_{f}=n_{f} \frac{2 \pi}{\lambda}$ and $k_{s}=n_{s} \frac{2 \pi}{\lambda}$ and $n_{s}>n_{f}$ the refractive indices along the axis and $\lambda$ the wavelength in vacuum. Therefore, if the electric field components of the incident radiation is given by $E_{1}=\varepsilon_{1} e^{-i \omega t}$ and $E_{2}=\varepsilon_{2} e^{-i \omega t}$ then, at the entrance of the retarder

$$
E_{f}=\left(\varepsilon_{1} \cos \alpha+\varepsilon_{2} \sin \alpha\right) e^{-i \omega t}, \quad E_{s}=\left(-\varepsilon_{1} \sin \alpha+\varepsilon_{2} \cos \alpha\right) e^{-i \omega t}
$$

and at the exit of the retarder

$$
E_{f}=\left(\varepsilon_{1} \cos \alpha+\varepsilon_{2} \sin \alpha\right) e^{-i \omega t}, \quad E_{s}=\left(-\varepsilon_{1} \sin \alpha+\varepsilon_{2} \cos \alpha\right) e^{i \delta} e^{-i \omega t}
$$

where

$$
\delta=\left(n_{s}-n_{f}\right) \frac{2 \pi l}{\lambda}
$$

is the dephasing term between both components and $l$ is the length of the retarder. A retarder such that $\delta=\pi / 2$ is called a quarter-wave plate, and one such that $\delta=\pi$ is a half-wave plate. 
When the ray crosses the polariser, only the component of the electric vector along the acceptance axis is transmitted, that is

$$
E_{a}=\left\{\cos (\beta-\alpha)\left[\varepsilon_{1} \cos \alpha+\varepsilon_{2} \sin \alpha\right]+\sin (\beta-\alpha)\left[-\varepsilon_{1} \sin \alpha+\varepsilon_{2} \cos \alpha\right] e^{i \delta}\right\} e^{-i \omega t} .
$$

The intensity of the output beam measured by the detector is

$$
I_{\text {meas }}(\alpha, \beta, \delta)=\left\langle E_{a}^{*}(\alpha, \beta, t) E_{a}(\alpha, \beta, t)\right\rangle
$$

or introducing the definition of the Stokes parameters

$$
\begin{array}{r}
I_{\text {meas }}(\alpha, \beta, \delta)=\frac{1}{2}\{I+(Q \cos 2 \alpha+U \sin 2 \alpha) \cos [2(\beta-\alpha)]- \\
(Q \sin 2 \alpha+U \cos 2 \alpha) \sin [2(\beta-\alpha)] \cos \delta+ \\
V \sin [2(\beta-\alpha)] \sin \delta\} .
\end{array}
$$

The measured intensity is thus a linear combination of the four Stokes parameters of the input beam. Adequate values of $\alpha, \beta$ and $\delta$ allow to determine $I, Q, U$ and $V$.

Measurement of circular polarisation. For measurements of the Stokes $V$ parameter, a quarter-wave plate $\left(\delta=90^{\circ}\right)$ is needed. And, for example, keeping the polariser fixed at $\beta=0^{\circ}$ yields:

$$
I_{\text {meas }}(\alpha)=\frac{1}{2}\{I+Q \cos 2 \alpha+U \sin 2 \alpha \cos 2 \alpha-V \sin 2 \alpha\}
$$

and thus, $I$ and $V$ can be measured as

$$
\begin{aligned}
I & =I_{\text {meas }}\left(-45^{\circ}\right)+I_{\text {meas }}\left(45^{\circ}\right), \\
V & =I_{\text {meas }}\left(-45^{\circ}\right)-I_{\text {meas }}\left(45^{\circ}\right) .
\end{aligned}
$$

\subsection{The radiative transfer equation}

The study of the radiative transfer in stellar atmospheres is, basically, the study of the interaction between radiation and matter. Following the context representative of penumbral atmospheres, let us concentrate in the description of the equations and concepts for the case of a radiative medium (atmosphere) in the presence of magnetic fields, and let us refer to the extensive literature, e.g. Landi degl'Innocenti (1992) and del Toro Iniesta (2003), for a detailed and complete development of the theory of transfer of radiation.

If we initially consider the case of isotropic radiation, the amount of energy $\mathrm{d} E_{\nu}$ transported through an area $\mathrm{d} A$, at the location $r$ in a time interval $\mathrm{d} t$, in the frequency band $\mathrm{d} \nu$, into the solid angle $\mathrm{d} \Omega$ around the direction $l$ is given by

$$
\mathrm{d} E_{\nu}=I_{\nu}(\boldsymbol{r}, \boldsymbol{l}, t)(\boldsymbol{l} \cdot \boldsymbol{n}) \mathrm{d} A \mathrm{~d} t \mathrm{~d} \nu \mathrm{d} \Omega=I_{\nu}(x, y, z, \theta, \varphi, t) \cos \theta \mathrm{d} A \mathrm{~d} t \mathrm{~d} \nu \mathrm{d} \Omega .
$$

Here, $\boldsymbol{n}$ is the normal to $\mathrm{d} A$ and $\boldsymbol{l}$ is described by polar coordinates $(\theta, \varphi)$ with respect to $\boldsymbol{n} . I_{\nu}$ is the specific intensity or surface brightness and represents the macroscopic 
counterpart specifying the energy carried by a bunch of identical photons along the beam. Thus, it is the quantity to use in the formulation of the radiative transfer.

We can extend the concept of specific intensity to the concept of polarised radiation (anisotropic case) by means of the Stokes vector I defined in the previous section. Then, the radiative transfer equation (RTE) describes the variation of the radiation field I along the path through the atmosphere, $s$, as a sum of the various effects produced in the interaction between radiation and matter and it can be written as follows

$$
\frac{\mathrm{d} \mathbf{I}}{\mathrm{d} s}=-\mathbf{K I}+\mathbf{j}
$$

where $\mathbf{K}$ represents the propagation or absorption matrix and the vector $\mathbf{j}$ characterises the emissivity processes.

\subsubsection{The propagation matrix}

The propagation matrix $\mathbf{K}$ accounts for the removal of energy from the electromagnetic field (propagating light) by matter (atmosphere) by supplying heat (kinetic energy) to the material particles. To account quantitatively for this phenomenon one can start analysing the changes in the refractive index produced by a plane electromagnetic wave propagating within a stellar atmosphere. From this analysis, one finds that $\mathbf{K}$ can be expressed as sum of three matrices:

$$
\mathbf{K}=\left(\begin{array}{cccc}
\eta_{I} & 0 & 0 & 0 \\
0 & \eta_{I} & 0 & 0 \\
0 & 0 & \eta_{I} & 0 \\
0 & 0 & 0 & \eta_{I}
\end{array}\right)+\left(\begin{array}{cccc}
0 & \eta_{Q} & \eta_{U} & \eta_{V} \\
\eta_{Q} & 0 & 0 & 0 \\
\eta_{U} & 0 & 0 & 0 \\
\eta_{V} & 0 & 0 & 0
\end{array}\right)+\left(\begin{array}{cccc}
0 & 0 & 0 & 0 \\
0 & 0 & \rho_{V} & -\rho_{U} \\
0 & -\rho_{V} & 0 & 0 \\
0 & \rho_{U} & -\rho_{Q} & 0
\end{array}\right)
$$

The diagonal matrix correspond to the absorption matrix. It accounts for absorption phenomena: the Stokes parameters evolve the same way without changes in the polarisation state of the light. The second is known as dichroism matrix. It accounts for the different degrees of absorption suffered by the different polarised components of the beam. The third corresponds to the dispersion matrix: phase shifts during the propagation or magneto-optical effects transform different states of linear polarisation among themselves and into circular polarisation. Examples of magneto-optical effects are shown in Fig. 2.5 below and shortly described in Sec.2.4.3.

Initially, the expressions for the propagation matrix elements in a magnetised atmosphere can be derived from the equation of motion of a bound electron in the presence of a magnetic field under the classical assumption: the electron (with charge $-e_{0}$ and mass $m_{e}$, neglecting relativistic effects) is bound to oscillate around the nucleus with frequency $\nu_{0}$. In the case of a gas consisting of $N$ atoms per unit volume, the matrix elements read 


$$
\begin{array}{ll}
\eta_{I}=\frac{\pi e_{0}^{2} N}{2 m_{e} c}\left[\phi_{p} \sin ^{2} \theta+\frac{1}{2}\left(\phi_{b}+\phi_{r}\right)\left(1+\cos ^{2} \theta\right)\right] & \\
\eta_{Q}=\frac{\pi e_{0}^{2} N}{2 m_{e} c}\left[\phi_{p}-\frac{1}{2}\left(\phi_{b}+\phi_{r}\right)\right] \sin ^{2} \theta \cos \varphi & \rho_{Q}=\frac{\pi e_{0}^{2} N}{2 m_{e} c}\left[\psi_{p}-\frac{1}{2}\left(\psi_{b}+\psi_{r}\right)\right] \sin ^{2} \theta \cos \varphi \\
\eta_{U}=\frac{\pi e_{0}^{2} N}{2 m_{e} c}\left[\phi_{p}-\frac{1}{2}\left(\phi_{b}+\phi_{r}\right)\right] \sin ^{2} \theta \sin \varphi & \rho_{U}=\frac{\pi e_{0}^{2} N}{2 m_{e} c}\left[\psi_{p}-\frac{1}{2}\left(\psi_{b}+\psi_{r}\right)\right] \sin ^{2} \theta \sin \varphi \\
\eta_{V}=\frac{\pi e_{0}^{2} N}{2 m_{e} c}\left[\phi_{r}-\phi_{b}\right] \cos \theta & \rho_{V}=\frac{\pi e_{0}^{2} N}{2 m_{e} c}\left[\psi_{r}-\psi_{b}\right] \cos \theta
\end{array}
$$

The matrix elements are made up of the absorption $(\phi)$ and dispersion $(\psi)$ profiles characteristic of the medium and of the geometry of the problem in terms of $\theta$ and $\varphi$, i.e. the inclination and azimuth angles of the magnetic field with respect to the direction of propagation of the light.

\section{The absorption and dispersion profiles}

For the case of a simple Zeeman triplet, the absorption $\phi$ and dispersion $\psi$ profiles in terms of frequency read

$$
\begin{aligned}
\phi_{p}(\nu) & =\frac{1}{\pi} \frac{\Gamma}{\left(\nu_{0}-\nu\right)^{2}+\Gamma^{2}} & \psi_{p}(\nu) & =\frac{1}{\pi} \frac{\nu_{0}-\nu}{\left(\nu_{0}-\nu\right)^{2}+\Gamma^{2}} \\
\phi_{r}(\nu) & =\frac{1}{\pi} \frac{\Gamma}{\left(\nu_{0}-\nu_{L}-\nu\right)^{2}+\Gamma^{2}} & \psi_{r}(\nu) & =\frac{1}{\pi} \frac{\nu_{0}-\nu_{L}-\nu}{\left(\nu_{0}-\nu_{L}-\nu\right)^{2}+\Gamma^{2}} \\
\phi_{b}(\nu) & =\frac{1}{\pi} \frac{\Gamma}{\left(\nu_{0}+\nu_{L}-\nu\right)^{2}+\Gamma^{2}} & \psi_{b}(\nu) & =\frac{1}{\pi} \frac{\nu_{0}+\nu_{L}-\nu}{\left(\nu_{0}+\nu_{L}-\nu\right)^{2}+\Gamma^{2}}
\end{aligned}
$$

where $\Gamma$ is known as the damping constant and $\nu_{L}$ is the Larmor frequency

$$
\nu_{L} \equiv \frac{e_{0} B}{4 \pi m_{e} c} .
$$

The indices $b$ (blue) and $r$ (red) refer to the profiles displaced to larger $\left(\phi_{b}, \psi_{b}\right)$ and smaller $\left(\phi_{r}, \psi_{r}\right)$ frequencies by $\Delta \nu=\nu_{L}$ with respect to the un-shifted $\left(\phi_{p}, \psi_{p}\right)$ profiles centred at $\nu_{0}$. Note that the use of the $b, p, r$ terms is equivalent to applying the selection rules for electric dipole transitions given in Sec.2.1. $\Delta M_{J}=0$ characterised the un-shifted $\pi$-component $(p)$ and the $\Delta M_{J}=+1,-1$ cases the shifted components $\sigma(b)$ and $\sigma(r)$, respectively. The absorption profiles $\phi$ are Lorentzian profiles and the dispersion profiles $\psi$ are antisymmetric.

The given profiles as they have been introduced do not account for thermal motions of the atoms and ions of the medium. The effect of such thermal agitation is introduced by convolving the $\phi(\nu)$ and the $\psi(\nu)$ profiles with a Maxwellian distribution function whose typical width or Doppler width, $v_{D}$, is related to the temperature of the medium $T$ by

$$
v_{D} \equiv \sqrt{\frac{2 k T}{m_{A}}+\xi_{m i c}^{2}}
$$


where $k$ is the Boltzmann constant, $m_{A}$ the rest mass of the atom and $\xi_{m i c}$, or microturbulence velocity accounts for those motions on scales smaller than the mean free path of photons. In terms of frequency shifts we have

$$
v_{D} \approx \frac{\Delta \nu_{D} c}{\nu_{0}} .
$$

Another effect which influences the shape of the $\phi$ and $\psi$ profiles is the mean macroscopic velocity of the medium in the direction of the line of sight. Such general motion of the medium shifts the frequency of the photons (Doppler effect) by

$$
\Delta \nu_{\mathrm{LOS}} \equiv \nu_{0, \alpha} \frac{v_{\mathrm{LOS}}}{c}
$$

Taking both effects into account, we can express the absorption and dispersion profiles for a normal Zeeman triplet in the compact form as

$$
\phi_{\alpha}\left(u_{\alpha}, a\right)=\frac{1}{\sqrt{\pi}} H\left(u_{\alpha}-u_{\mathrm{LOS}}, a\right)
$$

and

$$
\psi_{\alpha}\left(u_{\alpha}, a\right)=\frac{1}{\sqrt{\pi}} F\left(u_{\alpha}-u_{\mathrm{LOS}}, a\right)
$$

where the reduced variables for the frequency shifts and the damping constant are

$u_{\alpha}=\frac{\nu_{0, \alpha}-\nu}{\Delta \nu_{\mathrm{D}}}=\frac{\lambda-\lambda_{0, \alpha}}{\Delta \lambda_{\mathrm{D}}}, \quad u_{\mathrm{LOS}}=\frac{\Delta \nu_{\mathrm{LOS}}}{\Delta \nu_{\mathrm{D}}}=-\frac{\Delta \lambda_{\mathrm{LOS}}}{\Delta \lambda_{\mathrm{D}}}, \quad a=\frac{\Gamma}{\Delta \nu_{\mathrm{D}}}=\frac{\Gamma \lambda_{0}^{2}}{c \Delta \lambda_{\mathrm{D}}}$,

with $\nu_{0, \alpha}=\nu_{0}+\alpha \nu_{L}$ and $\alpha=-1,0,+1$ for $r, p, b$. The Voigt and Faraday-Voigt functions are defined by

$$
\begin{aligned}
& H \equiv \frac{a}{\sqrt{\pi}} \int_{-\infty}^{+\infty} e^{-y^{2}} \frac{1}{(u-y)^{2}+a^{2}} \mathrm{~d} y \\
& F \equiv \frac{a}{\sqrt{\pi}} \int_{-\infty}^{+\infty} e^{-y^{2}} \frac{u-y}{(u-y)^{2}+a^{2}} \mathrm{~d} y .
\end{aligned}
$$

In the more general case of operating with a spectral line characterised by a Zeeman multiplet formed in a magnetic field $\boldsymbol{B}$, we must use the correct quantum mechanical treatment. One has to superpose, i.e. to sum up, the $p, r, b$ absorption processes of all $\Delta M=0, \pm 1$ transitions with their statistical weights and with their displacements. Let us present here only the representation of profiles approximated by those of an effective triplet whose splitting is that of a normal triplet times the effective Landé factor $g_{\text {eff }}$ (Eq. 2.9)

$$
\phi_{\alpha}\left(u_{\alpha}, a\right) \simeq \frac{1}{\sqrt{\pi}} H\left(u+\alpha g_{\mathrm{eff}} u_{B}-u_{\mathrm{LOS}}, a\right)
$$

and

$$
\psi_{\alpha}\left(u_{\alpha}, a\right) \simeq \frac{1}{\sqrt{\pi}} F\left(u+\alpha g_{\mathrm{eff}} u_{B}-u_{\mathrm{LOS}}, a\right)
$$

where 


$$
u=\frac{\nu_{0}-\nu}{\Delta \nu_{\mathrm{D}}} \quad \text { and } \quad u_{\mathrm{B}}=\frac{\nu_{L}}{\Delta \nu_{\mathrm{D}}}=-\frac{\lambda_{\mathrm{B}}}{\Delta \lambda_{\mathrm{D}}}
$$

where $\lambda_{\mathrm{B}}$, the Zeeman splitting, was defined by Eq.2.5.

\subsubsection{Emission processes}

We now introduce the $\mathbf{j}$ term and with it the emissive properties of the medium which can be expressed as a Stokes vector:

$$
\mathbf{j}=\left(j_{I}, j_{Q}, j_{U}, j_{V}\right)^{T}
$$

Since the formation of a large number of spectral lines in the solar photosphere is adequately described in conditions of local thermodynamic equilibrium (LTE), departures from such equilibrium will be neglected and thus, strong simplifications can be applied to the general radiative transfer equation.

\section{Local thermodynamic equilibrium}

Under the LTE hypothesis it is assumed that only radiation, - and not matter -, is allowed to deviate from the thermodynamic equilibrium situation because of transport. Thus, the thermodynamic properties of matter are governed by the thermodynamic equations with local values of the temperature and density. Some consequences of the LTE approximation are then

a) local Maxwellian distribution of the velocities: the number of ions with velocities in the velocity interval $\mathrm{d} v$ around $v$ are given by

$$
N(v) \mathrm{d} v=\frac{N}{\sqrt{\pi} v_{D}} e^{-\left(v^{2} / v_{D}^{2}\right)} \mathrm{d} v,
$$

b) the local number of absorbers and emitters in the different quantum states are given by the Boltzmann equation for level population densities, i.e. the ratio of the atom densities $n$ in excitation levels $u$ and $l$

$$
\left\{\frac{n_{u}}{n_{l}}\right\}_{\mathrm{LTE}}=\frac{g_{u}}{g_{l}} e^{-\left(\chi_{u}-\chi_{l}\right) / k T}
$$

and by the Saha equation for the population ratio between two successive ionisation stages $r$ and $r+1$

$$
\left\{\frac{N_{r+1}}{N_{r}}\right\}_{\mathrm{LTE}}=\frac{1}{N_{e}} \frac{U_{r+1}}{U_{r}}\left(\frac{2 \pi m_{e} k T}{h^{2}}\right)^{3 / 2} e^{-\chi_{r} / k T}
$$

where $m_{e}$ is the electron mass, $N_{e}$ the electron density, $h$ the Planck constant, $k$ the Boltzmann constant and $T=T_{e}$ the local temperature. The terms $g_{l}$ and $g_{u}$ are the statistical weights for both levels, and $U_{r}$ the partition function of the stage $r$, $U_{r} \equiv \sum g_{r} e^{-\chi_{r} / k T}$ and $\chi_{u}, \chi_{l}$ and $\chi_{r}$ the excitation energy of levels $u$ and $l$ and ionisation energy of stage $r$, respectively. 
c) Kirchhoff's principle allows to write the emission coefficient in the form

$$
\mathbf{j}^{T}=B_{\nu}(T)\left(\eta_{I}, \eta_{Q}, \eta_{U}, \eta_{V}\right)
$$

where $B_{\nu}(T)$ is the Planck function

$$
B_{\nu}(T)=\frac{2 h \nu^{3}}{c^{2}} \frac{1}{e^{h \nu / k T}-1} .
$$

Hence, the emission is considered to have the isotropic black-body form. The emissivity vector $\mathbf{j}$ includes only the $\eta$ terms which affect the modification of the total intensity $I$ of the light beam.

Collisions between the material particles are an efficient mechanism to impose the LTE conditions and therefore it is expected that the higher the density of the medium the closer is the medium to the LTE conditions. The solar photosphere can be studied, to a wide extent, under the LTE approximation which is not the case for the chromosphere of the Sun whose density is not high enough.

\subsubsection{The RTE for a magnetised atmosphere}

The radiative transfer equation including the emissivity term can now be written in matrix form as

$$
\frac{d}{d s}\left(\begin{array}{c}
I \\
Q \\
U \\
V
\end{array}\right)=-\left(\begin{array}{cccc}
\eta_{I} & \eta_{Q} & \eta_{U} & \eta_{V} \\
\eta_{Q} & \eta_{I} & \rho_{V} & -\rho_{U} \\
\eta_{U} & -\rho_{V} & \eta_{I} & \rho_{Q} \\
\eta_{V} & \rho_{U} & -\rho_{Q} & \eta_{I}
\end{array}\right)\left(\begin{array}{c}
I-B_{\nu}(T) \\
Q \\
U \\
V
\end{array}\right)
$$

To cast the RTE into a more useful form for the analysis of the spectral line formation, let us introduce some concepts and manipulations. Contributions from both continuumforming processes and pure line-forming processes have to be added in the propagation matrix, and thus, $\mathbf{K}=\mathbf{K}_{\text {cont }}+\mathbf{K}_{\text {line }}$ where

$$
\mathbf{K}_{\text {cont }}=\kappa_{\text {cont }} \mathbf{1} \quad \text { and } \quad \mathbf{K}_{\text {line }} \equiv \frac{N \pi e_{0}^{2} f}{m_{e} c} \boldsymbol{\Phi},
$$

where $\kappa_{\text {cont }}$ is the frequency-independent absorption coefficient for the continuum and 1 the unity matrix. $N$ is the total population of absorbers, $e_{0}$ the charge of the electron in absolute value, $m_{e}$ the electron mass, $f$ the oscillator strength, i.e. a quantummechanical correction factor, and $\Phi$ contains the normalised absorption and dispersion profiles $\phi\left(u_{\alpha}, a\right)$ and $\psi\left(u_{\alpha}, a\right)$. Therefore, the total propagation matrix turns out to be

$$
\mathbf{K}_{\text {cont }}=\kappa_{\text {cont }}\left(\mathbf{1}+\eta_{0} \mathbf{\Phi}\right)
$$

where, by definition, $\eta_{0}$ is the line-to-continuum absorption coefficient ratio,

$$
\eta_{0} \equiv \frac{\kappa_{\text {lin }}}{\kappa_{\text {cont }}}=\frac{N \pi e_{0}^{2} f}{m_{e} c \kappa_{\text {cont }}} .
$$


The continuum optical depth is then defined

$$
\tau_{c} \equiv \int_{s}^{s_{0}} \kappa_{\text {cont }} \mathrm{d} s
$$

where the origin, $\tau_{c}=0$ at $s=s_{0}$, corresponds to the location of the observer.

If we also introduce the source function vector

$$
\mathbf{S} \equiv\left(B_{\nu}(T), 0,0,0\right)^{T},
$$

the RTE can be written in terms of the new variables as

$$
\frac{\mathrm{d} \mathbf{I}}{\mathrm{d} \tau_{c}}=\mathbf{K}(\mathbf{I}-\mathbf{S})
$$

where the symbol $\mathbf{K}$ has been kept for the propagation matrix. Thus, the matrix $\mathbf{K}$ elements follow now the form

$$
\begin{aligned}
\eta_{I} & =1+\frac{\eta_{0}}{2}\left\{\phi_{p} \sin ^{2} \theta+\frac{1}{2}\left[\phi_{b}+\phi_{r}\right]\left(1+\cos ^{2} \theta\right)\right\}, \\
\eta_{Q} & =\frac{\eta_{0}}{2}\left\{\phi_{p}-\frac{1}{2}\left[\phi_{b}+\phi_{r}\right]\right\} \sin ^{2} \theta \cos \varphi, \\
\eta_{U} & =\frac{\eta_{0}}{2}\left\{\phi_{p}-\frac{1}{2}\left[\phi_{b}+\phi_{r}\right]\right\} \sin ^{2} \theta \sin \varphi, \\
\eta_{V} & =\frac{\eta_{0}}{2}\left[\phi_{r}-\phi_{b}\right] \cos \theta
\end{aligned}
$$

and

$$
\begin{aligned}
& \rho_{Q}=\frac{\eta_{0}}{2}\left\{\psi_{p}-\frac{1}{2}\left[\psi_{b}+\psi_{r}\right]\right\} \sin ^{2} \theta \cos \varphi, \\
& \rho_{U}=\frac{\eta_{0}}{2}\left\{\psi_{p}-\frac{1}{2}\left[\psi_{b}+\psi_{r}\right]\right\} \sin ^{2} \theta \sin \varphi, \\
& \rho_{V}=\frac{\eta_{0}}{2}\left[\psi_{r}-\psi_{b}\right] \cos \theta .
\end{aligned}
$$

\subsection{Solutions of the RTE and magnetic field measure- ments}

\subsubsection{Integration of the RTE: the DELO method}

For the numerical integration of the radiative transfer equation (Eq.2.35) the DELO (Diagonal Element Lambda Operator) method by Rees et al. (1989) is used. It consists in introducing a modified propagation matrix $\mathbf{K}^{\prime}=\mathbf{K} / \eta_{I}-\mathbf{1}$ with zeros on its diagonal and a modified source vector $\mathbf{S}^{\prime}=\mathbf{j} / \eta_{I}$ and, thus, for $\tau \equiv \tau_{c}$ the RTE reads as

$$
\frac{\mathrm{d} \mathbf{I}}{\mathrm{d} \tau}=\mathbf{I}-\mathcal{S}
$$


where $\mathcal{S}=\mathbf{S}^{\prime}-\mathbf{K}^{\prime} \mathbf{I}$. We use $N$ grid points through the atmosphere with point number 1 at the top. The solution of this equation between two consecutive points of the grid, $\tau_{k}$ and $\tau_{k+1}$ for $k=1, \ldots, N-1$, is

$$
\mathbf{I}\left(\tau_{k}\right)=e^{-\left(\tau_{k+1}-\tau_{k}\right)} \mathbf{I}\left(\tau_{k+1}\right)+\int_{\tau_{k}}^{\tau_{k+1}} e^{-\left(\tau-\tau_{k}\right)} \mathcal{S}(\tau) \mathrm{d} \tau
$$

Here, a linear approximation for $\mathcal{S}$ it is introduced

$$
\mathcal{S}(\tau)=\left[\left(\tau_{k+1}-\tau\right) \mathcal{S}_{k}+\left(\tau-\tau_{k}\right) \mathcal{S}_{k+1}\right] /\left(\tau_{k+1}-\tau_{k}\right) .
$$

The emergent Stokes vector for every grid point can be expressed, after some algebra, as

$$
\mathbf{I}\left(\tau_{k}\right)=\mathcal{P}_{k}+\mathcal{D}_{k} \mathbf{I}\left(\tau_{k+1}\right)
$$

where

$$
\begin{aligned}
& \mathcal{P}_{k}=\left[\mathbf{1}+\left(F_{k}-G_{k}\right) \mathbf{K}_{k}^{\prime}\right]^{-1}\left[\left(F_{k}-G_{k}\right) \mathbf{S}_{k}^{\prime}+G_{k} \mathbf{S}_{k+1}^{\prime}\right] \\
& \mathcal{D}_{k}=\left[\mathbf{1}+\left(F_{k}-G_{k}\right) \mathbf{K}_{k}^{\prime}\right]^{-1}\left[e^{-\left(\tau_{k+1}-\tau_{k}\right)} \mathbf{1}-G_{k} \mathbf{K}_{k+1}^{\prime}\right]
\end{aligned}
$$

and

$$
\begin{aligned}
F_{k} & =1-e^{-\left(\tau_{k+1}-\tau_{k}\right)}, \\
G_{k} & =\left[1-\left(1+\tau_{k+1}-\tau_{k}\right) e^{-\left(\tau_{k+1}-\tau_{k}\right)}\right] /\left(\tau_{k+1}-\tau_{k}\right) .
\end{aligned}
$$

We assume no inward radiation at the surface or $\mathbf{I}^{-}(0)=\mathbf{0}$, and deep in the atmosphere the asymptotic (diffusion) approximation

$$
\mathbf{I}^{+}\left(\tau_{N}\right)=\left(B_{\nu}-\mathbf{K}^{-1} \frac{d B_{\nu}}{d \tau}\right) \mathbf{e}_{0}
$$

as boundary condition, with $\mathbf{e}_{0}=(1,0,0,0)^{T}$. Now, equation (2.44) can be calculated recursively to obtain the emergent Stokes vector at the surface or $\mathbf{I}(0)=\mathbf{I}\left(\tau_{1}\right)$.

\section{Macroturbulence velocity}

The observed Stokes profiles often appear to be wider than their equivalent synthetic profiles. This effect is due to the presence of macroscopic motions on scales larger than the mean free path of photons but unresolved in the spatial resolution element. In the astrophysical literature it is often called macroturbulence, and it is often assumed to be constant with depth. For analytical simplicity the macroscopic motions are assumed to have a Gaussian distribution of velocities with the parameter $\xi_{m a c}$. Their effect is then introduced as a convolution of the Stokes profiles with this Gaussian in terms of wavelength displacements:

$$
G_{m a c}=\frac{1}{\sqrt{\pi} \sigma_{m a c}} e^{-\left(\left(\lambda-\lambda_{0}\right)^{2} / \sigma_{m a c}^{2}\right)}
$$

where

$$
\sigma_{m a c}=\frac{\lambda_{0} \xi_{m a c}}{c} .
$$




\subsubsection{Stokes profiles}

Once the RTE is integrated and the macroturbulence effects have been taken into account, we dispose of the synthetic Stokes profiles of the emergent radiation that characterise the modelled atmosphere. The Stokes profiles show many interesting properties which are very helpful to extract information about the magnetic fields which cause the polarisation of the radiation field:

- Stokes $I$ and $V$ do not depend on the azimuthal angle $\varphi$ while Stokes $Q$ and $U$ do (Eqs.2.39]. Hence, the linearly polarised components are needed to fully characterise $B$.

- The Stokes $V$ parameter changes its sign if the component along the line of sight of the magnetic field changes in polarity. The dependence of $V$ with $\cos \theta$ (Eqs. 2.39. where $\theta$ is the angle between the LOS and $\boldsymbol{B}$ ) is the reason. This reversal of the $V$ lobes will be a diagnostic for the changes in polarity of $B_{\mathrm{LOS}}$ in the observed structures.

- In the presence of gradients of velocity along the atmosphere, the Stokes $V$ looses its anti-symmetry, i.e. the blue and red lobes are different in amplitude. These asymmetries may also be caused by a mixture of changes of velocity and $\boldsymbol{B}$ along the LOS. The origin of such asymmetries in penumbrae, as will be treated in detail in Chapter 5, lies in the dynamic and magnetic nature of the structures at small scales. Therefore, the synthesis or forward modelling, of Stokes profiles including such gradients will be a powerful tool to understand the observed Stokes profiles.

\subsubsection{Stokes $I$ and $V$ profiles with varying magnetic field strength}

The radiation that reaches the observer is the result of photons that are emitted, absorbed and re-emitted or scattered while travelling along and interacting with the medium. Thus, it is conceivable that different photons at various wavelengths are "formed" in different parts of the medium. For the solar atmosphere, it is useful then to perform observations in different spectral lines in order to learn about the physical properties at different depths of the medium. As an example, Stokes $I$ and $V$ for three different spectral lines synthesised for the same atmosphere model assumptions are shown next. They possess different characteristics in line depression and width. The response of such lines to variations of the magnetic field strength can also be seen in this figure.

Figure 2.5 depicts, for three spectral absorption lines, the behaviour of the Stokes $I$ and $V$ profiles with increasing magnetic field strength from 0 Gauss (dark blue) up to 3200 Gauss (orange). The LOS is parallel to the vertical in the atmosphere and an inclination angle $\gamma=45^{\circ}$ with respect to the vertical is considered for the magnetic field. Thus, a case intermediate to the longitudinal and the transverse Zeeman effect is treated. The synthetic profiles for the different spectral lines under study are calculated from a mean penumbral model by del Toro Iniesta et al. (1994). The left panels depict the profiles of the Fe I $6301.5 \AA$ line. The strongest magnetic fields cause a broadening of the intensity profile of this line which is not strongly sensitive to magnetic fields ( $\left.g_{\text {eff }}=1.67\right)$ while the Stokes $V$ profiles increase in amplitude, become saturated at about 1900 Gauss and the 

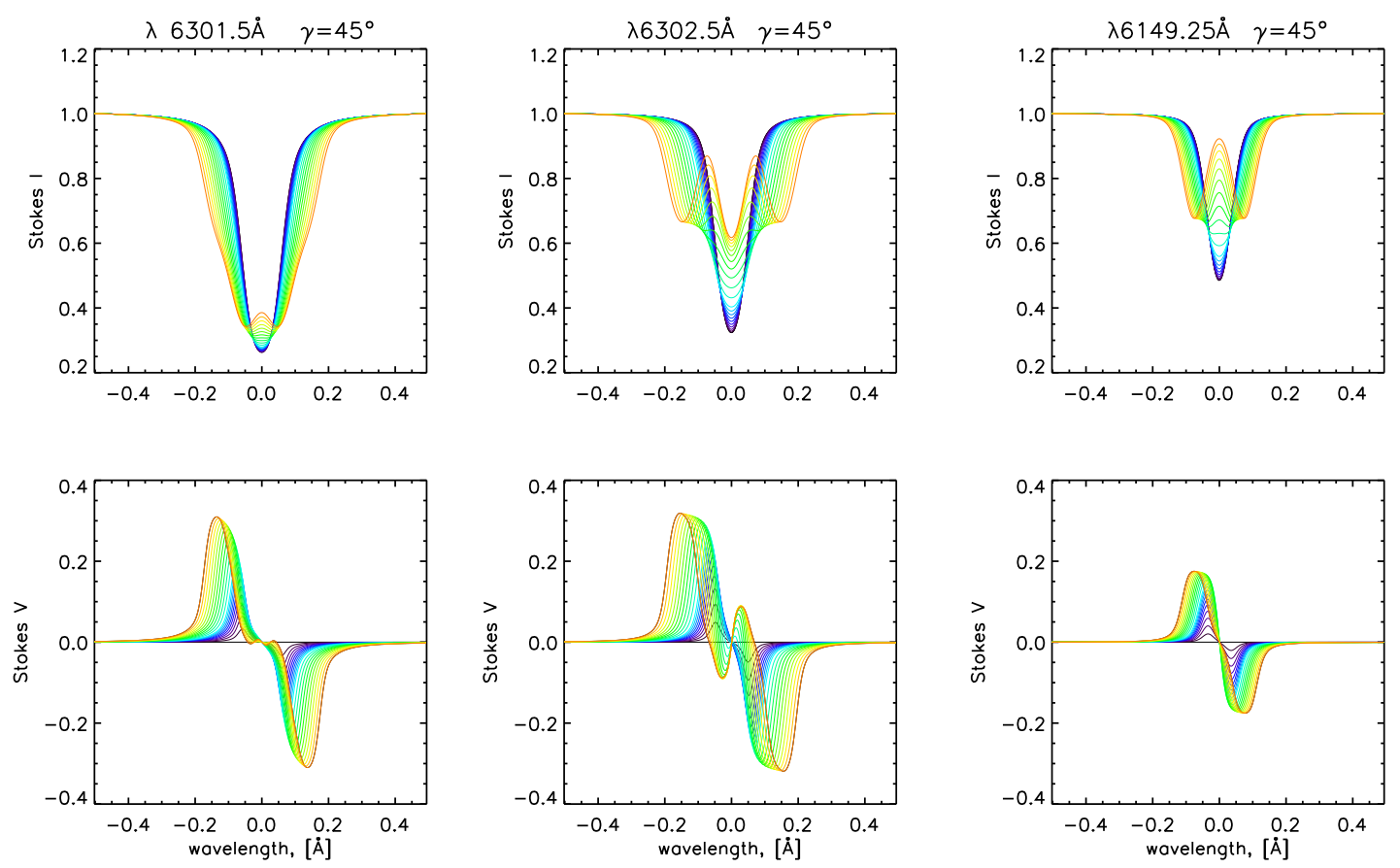

Figure 2.5: Synthetic Stokes $I$ and $V$ profiles for the lines discussed above and used in the study. The change of colours from dark blue to orange indicates an increase of the magnetic field strength from 0 to 3200 Gauss. The $I$ and $V$ values are relative to the continuum intensity, $I_{c}$. $\gamma$ is the inclination angle of $\mathbf{B}$ with respect to the vertical direction in the model atmosphere.

extrema separate up to $280 \mathrm{~m} \AA$ at 3200 Gauss. For the Fe I $6302.5 \AA$ line (central panels of Fig.2.5, the Zeeman triplet can be clearly seen at field strengths of 2100 Gauss and larger. The Stokes $V$ profiles reach the saturation at approximately 1500 Gauss, then the separation of the $V$ extrema becomes the dominant effect with increasing field strength and reaches $325 \mathrm{m \AA}$ at 3200 Gauss. The $V$ profiles show small lobes with opposite sign close to the central wavelength. They are associated with magneto-optical effects introduced through the anti-symmetrical terms of the propagation matrix (Sec.2.3.1). The magneto-optical effects are important for a correct interpretation of spectropolarimetric data from sunspots, as pointed out by Wittmann (1971). The Fe II $6149.3 \AA$ line (right panels in Fig.2.5) exhibits a separation of its $\sigma$ components in the intensity (Stokes $I$ ) profiles, starting at field strengths of 1600 Gauss and the amplitudes of the Stokes $V$ extrema reach 0.18 of the continuum intensity $I_{c}$, in this case. The values of the magnetic field strengths at which the $\sigma$-components become visible in $I$ and the $V$ extrema start separating depends on the width of the spectral line, most importantly on the Doppler broadening by thermal and non-thermal motions. The above modelling of synthetic profiles demonstrates the behaviour of the Stokes parameters in a penumbral atmosphere which was assumed static (velocities set to zero) and with constant magnetic fields.

The measurements of the magnetic fields from observations are based on calibrations using the solutions of the radiative transfer equations under different approximations. The various considerations and methods of calibration of the magnetic field are next introduced. 


\subsubsection{Weak field approximation}

The weak field approximation (WFA) considers that broadening caused by the magnetic field $\left(\Delta \lambda_{B}\right)$ is much smaller than the Doppler broadening $\left(\Delta \lambda_{D}\right)$,

$$
\Delta \lambda_{\mathrm{B}} \ll \Delta \lambda_{D}=\sqrt{\frac{2 k T}{m_{A}}} \frac{\lambda}{c}
$$

with $k$ the Boltzmann constant, $T$ the gas temperature and $m_{A}$ the atomic mass. In this case it is not possible to detect from the splitting of the intensity profiles the effect caused by the magnetic field. Under this assumption and following Landi degl'Innocenti (1992) we can expand the absorption and dispersion profile equations in series up to first order in $\Delta \lambda_{B}$ :

$$
\begin{array}{ll}
\phi_{p}\left(\lambda-\lambda_{0}\right)=\phi_{0}\left(\lambda-\lambda_{0}\right), & \phi_{r, b}\left(\lambda-\lambda_{0}\right)=\phi_{0}\left(\lambda-\lambda_{0}\right) \mp \Delta \lambda_{B} \frac{\partial \phi_{0}\left(\lambda-\lambda_{0}\right)}{\partial \lambda}, \\
\psi_{p}\left(\lambda-\lambda_{0}\right)=\psi_{0}\left(\lambda-\lambda_{0}\right), & \psi_{r, b}\left(\lambda-\lambda_{0}\right)=\psi_{0}\left(\lambda-\lambda_{0}\right) \mp \Delta \lambda_{B} \frac{\partial \psi_{0}\left(\lambda-\lambda_{0}\right)}{\partial \lambda},
\end{array}
$$

where $\phi_{0}$ and $\psi_{0}$ are the profiles relative to the line centre $\lambda_{0}$. The propagation matrix elements read then

$$
\begin{array}{cc}
\eta_{I}=1+\eta_{0} \phi_{0}\left(\lambda-\lambda_{0}\right), & \eta_{Q}=\eta_{U}=\rho_{Q}=\rho_{U}=0 \\
\eta_{V}=-\eta_{0} \Delta \lambda_{\mathrm{B}} \cos \theta \frac{\partial \phi_{0}\left(\lambda-\lambda_{0}\right)}{\partial \lambda}, & \rho_{V}=-\eta_{0} \Delta \lambda_{\mathrm{B}} \cos \theta \frac{\partial \psi_{0}\left(\lambda-\lambda_{0}\right)}{\partial \lambda}
\end{array}
$$

Substituting these in the radiative transfer equations (Eq.2.35) for the Stokes parameters we have

$$
\frac{d I}{d \tau_{c}}=\eta_{I}\left(I-B_{T}\right)+\eta_{V} V, \quad \frac{d V}{d \tau_{c}}=\eta_{I} V+\eta_{V}\left(I-B_{T}\right) .
$$

The equations for $Q$ and $U$ have no source term. Since, deep in the atmosphere, the radiation is unpolarised then $Q=U=0$ through the whole atmosphere. Also, the $\eta_{V} V$ term is of the order of $\left(\Delta \lambda_{B}\right)^{2}$ and therefore negligible. Thus, the equation for the intensity $I$

$$
\frac{d I}{d \tau_{c}}=\left(1+\eta_{I}\right)\left(I-B_{T}\right) \equiv \frac{d I_{0}}{d \tau_{c}}
$$

and for the Stokes $V$ parameter

$$
\frac{d V}{d \tau_{c}}=\left(1+\eta_{I}\right) V-\eta_{0} \Delta \lambda_{B} \cos \theta \frac{\partial \phi_{0}\left(\lambda-\lambda_{0}\right)}{\partial \lambda}\left(I_{0}-B_{T}\right)
$$

Equation 2.46tells us that the intensity of a line formed in the presence of a weak magnetic field can be approximated by the intensity of the same line formed in the same atmosphere but in the absence of magnetic field, $I_{0}$. It is now straight forward to see that if we take the spectral derivative of the intensity, equivalent to that of $I_{0}$, and multiply by a $-\Delta \lambda_{B} \cos \theta$ term we obtain the equation 
$\frac{d}{d \tau_{c}}\left(-\Delta \lambda_{B} \cos \theta \frac{d I_{0}}{d \lambda}\right)=\left(1+\eta_{I}\right)\left(-\Delta \lambda_{B} \cos \theta \frac{d I_{0}}{d \lambda}\right)-\eta_{0} \Delta \lambda_{B} \cos \theta \frac{\partial \phi_{0}\left(\lambda-\lambda_{0}\right)}{\partial \lambda}\left(I_{0}-B_{T}\right)$

If we now compare equations (2.47) and (2.48) we find identical differential equations for $V$ and for $-\Delta \lambda_{B} \cos \theta \frac{d I_{0}}{d \lambda}$. Therefore we conclude that

$$
V=-\Delta \lambda_{B} \cos \theta \frac{\mathrm{d} I_{0}(\lambda)}{\mathrm{d} \lambda}
$$

or writing $\Delta \lambda_{B}$ explicitely (Eq.2.8), we arrive at the well known formula

$$
V(\lambda)=-4.67 \times 10^{-13} g_{\text {eff }} \lambda_{0}^{2} B_{\text {eff }} \frac{\mathrm{d} I_{0}(\lambda)}{\mathrm{d} \lambda},
$$

when $B_{\text {eff }}$ is measured in Gauss and wavelength $\lambda_{0}$ in $\AA$. $I_{0}(\lambda)$ is the un-split, i.e. to first order magnetically unaffected, Stokes $I$ profile in the observed magnetic structure on the Sun. The effective magnetic field strength is given by

$$
B_{\text {eff }}=\alpha \cos \theta B \text {, }
$$

with a factor $\alpha<1$ accounting for parasitic light and $\theta$ the angle between the magnetic field and the LOS. The parasitic light may be stray light caused by scattering in Earth's atmosphere and telescope optics and it may stem from non-magnetic solar structures contained in the spatial resolution element during observation.

The WFA applies only for lines with moderate to small Landé factors, $g_{\text {eff }}$, like the Fe II $6149.3 \AA$ and the Fe I $6301.5 \AA$ Alines, and in weak to moderate magnetic fields of strength of $B<1000 \mathrm{G}$ for the two above lines. For stronger fields the strengths determined with the WFA are systematically underestimated.

\subsubsection{Strong field regime}

Opposite to the WFA described above, in the presence of strong magnetic fields or when spectral lines strongly sensitive to the magnetic field are used, the strong field regime (SFR) is considered. In this case, the spectral lines undergo strong Zeeman splitting and the $V$ profiles have reached the point of saturation. According to Fig.2.5, the $V$ amplitudes first increase with the magnetic field strength until some value of $B$ is reached at which the $V$ amplitudes cease increasing and the extrema of the $V$ lobes start to separate. Then, it is possible to make estimates of the total magnetic field strength from the separation of the lobes or Zeeman splitting.

The formula for the Zeeman splitting $\left(\Delta \lambda_{\mathrm{B}}\right)$ gives

$$
\Delta \lambda_{\mathrm{B}}=4.67 \times 10^{-13} g_{\mathrm{eff}} \lambda_{0}^{2} B,
$$

$B$ can be measured from the separation $2 \Delta \lambda_{\mathrm{B}}$ of the $V$ extrema. It depends neither on the stray light nor on the angle $\theta$. In the case of the FeI $6302.5 \AA$ line, with $g=2.5$, 

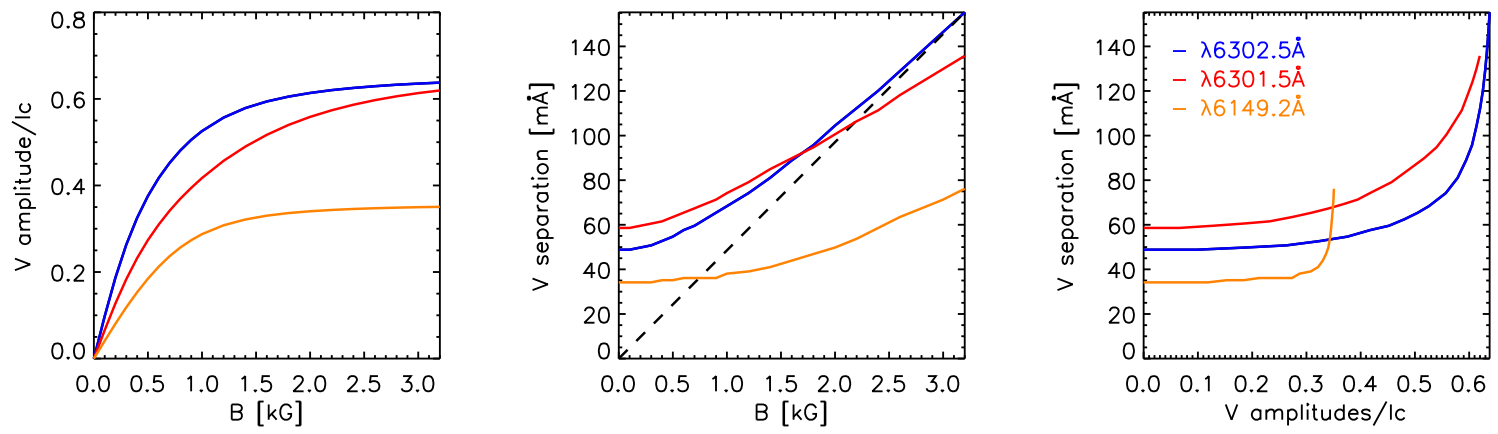

Figure 2.6: Left: Stokes $V$ amplitudes (from peak to peak, relative to continuum intensity $I_{c}$ ) vs. magnetic field strength. Middle: Stokes $V$ separation vs. B. Right: $V$ separations vs. $V$ amplitudes. All three panels correspond to values of $B$ from 0 to 3200 Gauss and for three different spectral lines. For more information see the text.

for sufficiently strong magnetic fields $\left(\Delta \lambda_{\mathrm{B}}>\Delta \lambda_{\mathrm{D}}\right.$, or approximately $B>1500$ Gauss) the separation of the $V$ extrema starts to increase linearly with $B$ while the amplitude of Stokes $V$ becomes independent of $B$.

The left panel of Fig.2.6 shows the $V$ amplitudes vs. the magnetic field strengths considered in the model atmosphere of a sunspot penumbra by del Toro Iniesta et al. (1994) already used above. The inclination angle $\gamma$ is assumed to be $45^{\circ}$ again. The amplitudes of $V$ increase with $B$ until some value where they become independent of the magnetic field strength. This effect is most clearly seen in the spectral line at $6149.3 \AA$ (orange). The middle panel shows the separation of the $V$ extrema vs. $B$. For all lines, an increase of the $V$ separation with $B$ can be seen. However, the separation for the Fe II line does not show a linear dependence below 1800 Gauss which tells us that the SFR cannot be used in this regime for the estimations of magnetic fields from observations in this line. For field strengths below approximately 800 Gauss we can apply the WFA. For field strengths between 800 and 1800 one needs calibration curves like the ones in Fig.2.6, For strong $B$, as expected in sunspot penumbrae, the separation of the $V$ extrema of the $6302.5 \AA$ line increases almost linearly with $B$. For measurements of the magnetic fields from observations in this line, the SFR will be applied. For the case of the $6301.5 \AA$ line we will apply the WFA, although, as it will be seen later, observations in this line will be mostly used for the measurement of intensities and of LOS velocities. On the right panel of Fig.2.6 the separations of the $V$ extrema vs. their amplitudes show how both quantities depend on $B$ in different regimes. The highest values of separation of $V$ extrema and saturation of $V$ extrema are reached by the magnetically sensitive $\lambda 6302.5 \AA$ line. The lowest values stem from $\lambda 6149.3 \AA$ with $g_{\text {eff }}=1.33$.

\subsubsection{Centre of gravity method}

An efficient method for determining the longitudinal component of the magnetic field is the centre of gravity (COG) method. Semel (1967) and Rees \& Semel (1979) have shown that the wavelength separation of the COG of the profiles $\frac{1}{2}\left(I_{\lambda}+V_{\lambda}\right)$ and $\frac{1}{2}\left(I_{\lambda}-V_{\lambda}\right)$ gives the average field component along the LOS. Here, the average means a mean value 
over the height of the line formation in the atmosphere and over the area of the spatial resolution element.

The COG of each of the two profile components is defined as the centroid of the respective intensity profiles:

$$
\lambda_{\mathrm{COG}, \pm}=\frac{\int \lambda\left[I_{c}-\left(I_{\lambda} \pm V_{\lambda}\right)\right] \mathrm{d} \lambda}{\int\left[I_{c}-\left(I_{\lambda} \pm V_{\lambda}\right)\right] \mathrm{d} \lambda}
$$

where $I_{c}$ is the continuum intensity. The determination of the centroids is completely unaffected by spectral broadening. The longitudinal component of the magnetic field (in Gauss) is then determined through the relation

$$
\frac{\lambda_{\mathrm{COG},+}-\lambda_{\mathrm{COG},-}}{2}=4.67 \times 10^{-13} g_{\mathrm{eff}} \lambda_{0}^{2} B_{\mathrm{LOS}}
$$

where, again, $\lambda_{0}$ is the central wavelength of the line in $\AA, g_{\text {eff }}$ the effective Lande factor and $B_{\mathrm{LOS}}=B \cos \theta$, with $\theta$ the angle between the LOS and $\boldsymbol{B}$.
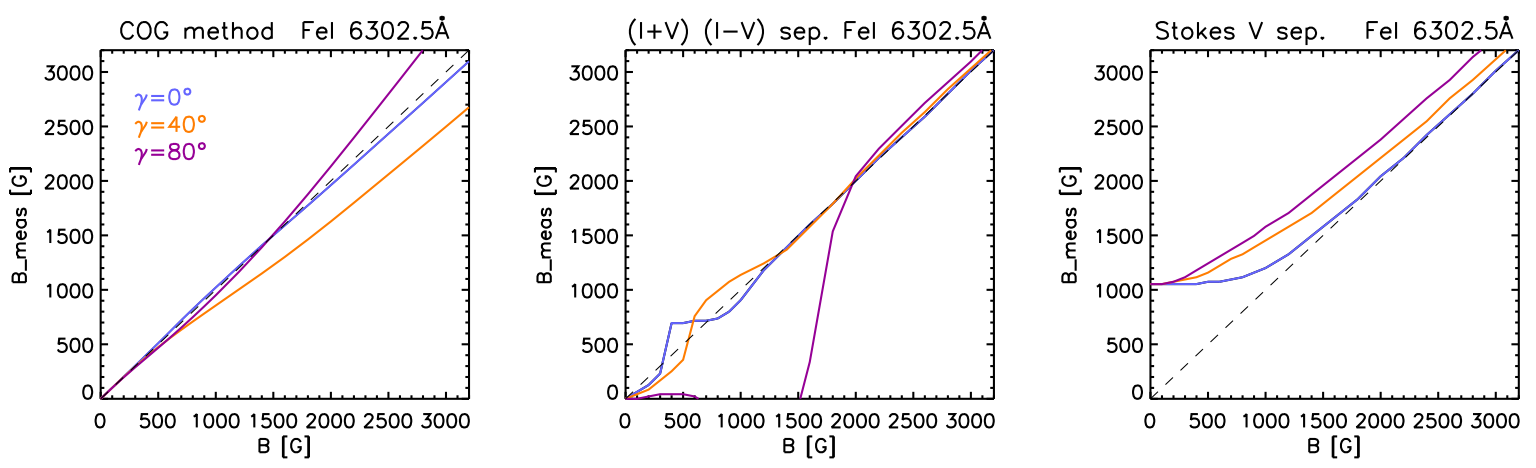

Figure 2.7: 'Measured' magnetic field strength $B_{\text {meas }}$ vs. input values of magnetic field strength $B$, numerical simulations for Fe I 6302.5. Left: from the COG method. Middle: from separation of minima of circularly polarised profiles, $1 / 2\left(I_{\lambda} \pm V_{\lambda}\right)$. Right: from separation of Stokes $V$ extrema. Dashed line shows a one-to-one correspondence.

Some calculations with the COG method have been performed to test the accuracy of the method applied to synthetic profiles of the line at $6302.5 \AA$ : For vertical LOS and for different inclination angles, the magnetic field strength has been estimated using Eq. 2.53, Once more, the profiles used for that purpose have been synthesised from a mean penumbral atmosphere. The results for three different angles are shown in Fig.2.7(left). There, 'measured' magnetic field strengths $B_{\text {meas }}$ vs. (real) magnetic field strengths $B$ used as input for the modelling are plotted. The factor $\cos \gamma$ was assumed to be known in the 'measurements'. Therefore, total strengths are represented. The dashed straight line gives a one-to-one correspondence. For longitudinal magnetic fields $\left(\gamma=0^{\circ}\right)$, the estimation is almost exact, slightly underestimating the highest values. For angles around $40^{\circ}$ and within the considered range of field strengths, the underestimation is of the order of 400 Gauss. And for $\gamma$ around $80^{\circ}$ and for $B>1500$ Gauss the field strength is overestimated 
gradually up to $\sim 400$ Gauss.

For comparison, tests have been made for measurements from the separation of the minima of both polarised components as well as from the separation of the Stokes $V$ extrema (Fig.2.7 middle and right panels ). For the former, there is a good correspondence for inclination angles up to $60^{\circ}$ from 1300 Gauss on. For more transverse fields, the determinations only fit from 2000 Gauss on. From the separation of the $V$ extrema, for values smaller than 1000 Gauss, $B_{\text {meas }}$ come out too large. For $B$ larger than 1000 Gauss, measured magnetic fields are also overestimated following a nearly linear behaviour with an offset increasing with $\gamma$, up to 500 Gauss for $\gamma=80^{\circ}$. 



\section{Observations and data reduction}

This chapter deals with the description of the observations realized during the different campaigns and the processing of the data which are the basis of this thesis work.

The German Vacuum Tower Telescope (VTT) located in the Obervatorio del Teide (2400 $\mathrm{m}, 16^{\circ} 30^{\prime}$ West, $28^{\circ} 18^{\prime}$ North) in Tenerife, is equipped with a coelostat which directs the Sun's light to the main mirror $(70 \mathrm{~cm})$ in the interior of the evacuated telescope tank. The evacuation down to 1 mbar avoids turbulences from warmed-up air. A series of flat mirrors redirects the light achieving an effective focal length of $46 \mathrm{~m}$, an image scale of $4.6 / \mathrm{mm}$ and a diffraction limited spatial resolution at $6000 \AA$ of $\lambda / D=0^{\prime \prime} 177$.

\section{1 'Göttingen' FPI data}

The 'Göttingen' Fabry-Pérot interferometer (FPI) is a suitable optical setup to perform two-dimensional (2D) speckle spectroscopy and polarimetry. It was built by the Universitäts-Sternwarte in Göttingen (Germany) in the early 1990s (Bendlin et al. 1992, Bendlin \& Volkmer 1995) and it is mounted at the VTT. A general schema of the spectrometer is shown in Fig. 3.1.

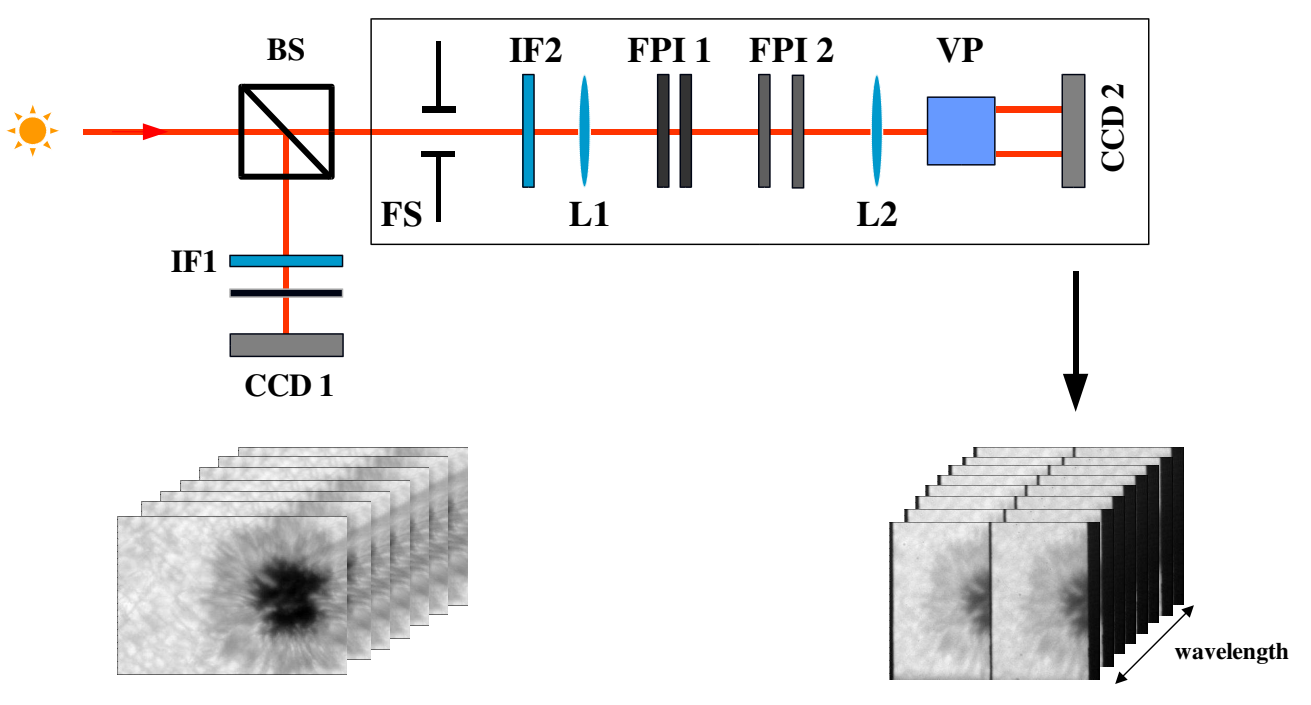

Figure 3.1: Basic schema of optical setup of the 'Göttingen' FPI: $B S$, beam splitter; $N F$, neutral density filter; $I F 1$ and $I F 2$, interference filters; $F S$, field stop; $L 1$, collimator lens; $L 2$, camera lens; FPI 1 and FPI 2, Fabry-Pérot etalons; VP, Stokes $V$ polarimeter; $C C D 1$ and $C C D 2$, detectors for the broad-band and narrow-band channel, respectively. 
The Sun's light coming from the telescope is directed to the spectrometer. A beam splitter (BS) takes out about $5 \%$ of the incoming light to the broad-band channel. The broad-band (BB) channel consists of an interference filter (IF1) with typical FWHM (Full Width at Half Maximum) of 50-100 $\AA$ and a proper neutral density glass $(N F)$ to filter out an excess of photons which would saturate the $\mathrm{CCD}$ detector. The $\mathrm{BB}$ channel provides us with integrated-light images of the solar surface, at the photosphere, needed for the restoration of the images distorted by the turbulence of the terrestrial atmosphere or 'seeing' as will be explained in Sec.3.2

The Fabry-Pérot interferometer. For spectroscopy and polarimetry, the narrow-band (NB) channel is equipped with two Fabry-Pérot interferometers which scan in wavelength. A Fabry-Pérot interferometer (FPI) consists of two parallel plates with reflecting surfaces of reflectance $R$ and transmittance $T$. A beam incident to the parallel plates, will be reflected several times. Upon each reflexion, part of the intensity will be transmitted to some fraction and all these transmitted fractions interfere in the outgoing beam giving rise to a final intensity distribution, the Airy function (see e.g. Born \& Wolf)

$$
I=I_{\max } \frac{1}{1+\frac{4 R}{(1-R)^{2}} \sin ^{2} \frac{\delta}{2}}
$$

where, neglecting absorption, $I_{\max }=T^{2} /(1-R)^{2}$, and the phase

$$
\delta=\frac{4 \pi}{\lambda} n d \cos \Theta \text {. }
$$

The varying transmission function of the FPI is caused by interference of the light rays, or waves, multiply reflected at the high-reflectivity surfaces. Constructive interference occurs if the transmitted beams are in phase, corresponding to a high-transmission peak. If the transmitted beams are out-of-phase, destructive interference occurs corresponding to a transmission minimum. Whether the multiply-reflected beams are in-phase or not depends on the wavelength $\lambda$ of the light, the angle $\Theta$ of the direction of the light with respect to the normal of the etalon, the spacing $d$ of the etalon plates and the refractive index $n$ of the material between the reflecting surfaces.

A second, broader (with larger free spectral range) FPI etalon is used to filter the transmission function of the narrower one in order to isolate one single transmission peak. Thus, the two tunable FPIs allow 2D imaging of the Sun at different positions of a given spectral line, preselected by a narrow interference filter (IF2) with typical FWHM of 3$10 \AA$.

Spectral resolution: The free spectral range (FSR) of the FPI gives the distance (in wavelength) between adjacent transmission peaks, which in terms of the observed wavelength $\lambda$ and the distance of the FPI plates $d$ is given by

$$
\mathrm{FSR}=\lambda^{2} / 2 n d
$$

with $n \approx 1$ the refraction index of air and $d_{\mathrm{FPI} 2}=1.5 \mathrm{~mm}$ and $d_{\mathrm{FPI} 1}=125 \mu \mathrm{m}$. After the multiplication of the three transmission functions - IF transmission and both FPI 1 and 
FPI 2 Airy functions - the resulting function will be characterised by the FWHM of the narrowest one as seen in Fig. 3.2

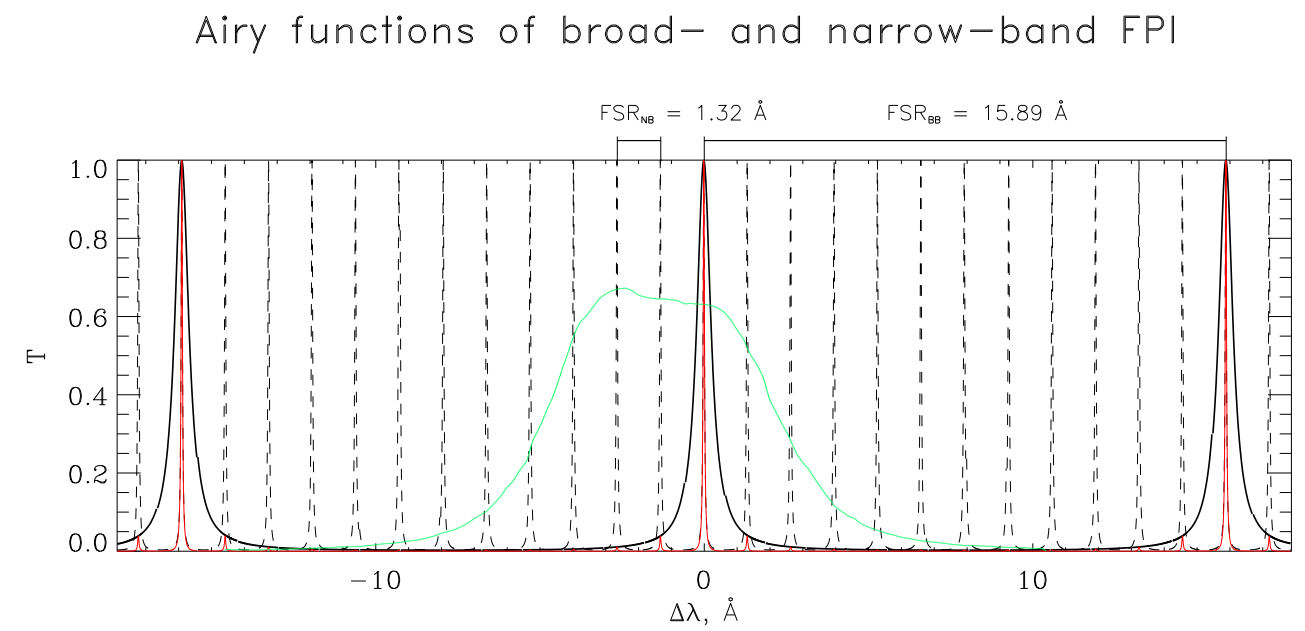

Figure 3.2: Transmission of the used interference filter (green curve), Airy function of the broadband FPI (black solid line), Airy function of the narrow-band FPI (dashed line). FSR and FWHM denote the Free Spectral Range and the Full Width at Half Maximum, respectively (from Okunev 2004).

The FWHM $(\Delta \lambda)$ is calculated from the ratio,

$$
\Delta \lambda=\mathrm{FSR} / \mathcal{F}
$$

where $\mathcal{F}$ is the finesse of the FPI, i.e. a figure of merit for the transmission bandwidth of the FPI. Imperfections in the reflecting surfaces, errors in parallelism, and imperfections in the material between the reflective surfaces will degrade the finesse.

The measured (and derived) values of these parameters for FPI 2 at the different spectral lines we use are

\begin{tabular}{cccc}
$\lambda(\AA)$ & FSR $(\AA)$ & $\mathcal{F}_{\text {eff }}$ & $\Delta \lambda(\mathrm{m \AA})$ \\
\hline 6149.3 & 1.26 & 30 & 42 \\
6301.5 & 1.32 & 30 & 44 \\
6302.5 & 1.33 & 30 & 44
\end{tabular}

Hence, the spectral resolution characteristic of our optical setup is $\lambda / \Delta \lambda \sim 140000$.

The choice of the sampling along a given spectral line, to reach the best compromise for a given observational purpose, will depend on various factors to take into account:

a) the narrow transmission of the spectrometer needed to perform spectroscopy and the short exposure time $(\sim 20-30 \mathrm{~ms})$ required for speckle imaging and speckle re- 
construction (see below), make it necessary to take more than one frame per spectral position in order to improve the signal to noise ratio,

b) for proper sampling of the line and to avoid strong oversampling, the step width of the sampling must be $\sim \Delta \lambda / 2$, and

c) the number of frames which can be taken in one scan (150) is restricted due to limitation in memory of the hardware in use 1 .

d) A data set or scan, consists, then, in narrow-band images from several wavelength positions in a spectral line with some 5-10 frames per position. Strictly simultaneously with the narrow-band images, broad-band images are taken. The lower part of Fig. 3.1 shows the type of data one obtains. The $V$ polarimeter in the narrow-band channel separates the images in left and right circularly polarised light.

In the next section the selected parameters for the observations of the data used in this work will be described.

\subsubsection{Description of the observations}

The observations were performed during different campaigns in April 2002 and May 2003. The seeing conditions were excellent for the first and moderate for the second ones. They correspond both to polarimetric data with the characteristics shown in Table 3.1. The full size of the field of view (FOV) ( $384 \times 256$ pixels) was used in both detectors, CCD 1 and CCD 2. The pixel size corresponds to $0 ! 1 \times 0 . \prime 1$, or approximately $76 \mathrm{~km} \times 76 \mathrm{~km}$ on the solar surface.

\begin{tabular}{ccccccc} 
Date & Spectral line $(\AA)$ & Exp. time & Step width & No. $\lambda_{\text {posit }}$ & No. im/posit. & Spectral range \\
\hline 29.04 .02 & Fe I $6301.5+6302.5$ & $30 \mathrm{~ms}$ & $31.8 \mathrm{~m} \AA$ & 33 & 5 & $410+410 \mathrm{~m} \AA$ \\
02.05 .03 & Fe II 6149.3 & $20 \mathrm{~ms}$ & $27.8 \mathrm{~m} \AA$ & 18 & 8 & $472 \mathrm{~m} \AA$
\end{tabular}

Table 3.1: FPI spectrometer settings with dates of observations, spectral lines, exposure times, scanning step widths, number of spectral positions in one scan, number of frames taken at each wavelength position and the total sampled spectral range. The telluric $\mathrm{O}_{2}$ line at $6302.8 \AA$ has been also scanned with five spectral positions for calibration purposes.

As it was mentioned in the previous section, the data of the BB and the NB channel are taken simultaneously, with the same exposure time and number of frames per scan. The cadence of each scan is about 50 seconds, including storage of the data which is shorter than the time scale of variation of the solar structures under analysis. In the case of the data obtained at $\lambda 6149.3 \AA$, several scans were taken and thus, a study of the time evolution of the penumbral structure during the observing time can be performed.

\footnotetext{
${ }^{1}$ The hardware and software have been recently upgraded to the new "Göttingen" Fabry-Pérot Spectrometer (FPS) including a new etalon with improved spectral resolution.
} 
The positions of the sunspots corresponded to a low heliocentric angles, $\theta \approx 20^{\circ}$ $(\mu=\cos \theta=0.93)$ for NOAA 9919 and $\theta \approx 23^{\circ}(\mu=0.92)$ for NOAA 0346. Thus, the sunspots were not located far from the solar disc centre 2 . It is important to note that the viewing angle of the observations plays an important role when studying three-dimensional structures on the Sun. In the case of sunspots it is common to distinguish between centreside (CS) and limb-side (LS) penumbra, meaning with centre and limb side the part of the sunspot nearer to the disc centre and further away from the disc centre, respectively. The relevance of the projection effects will be explained in detail when discussing the results obtained from these data (Sec.4.1.
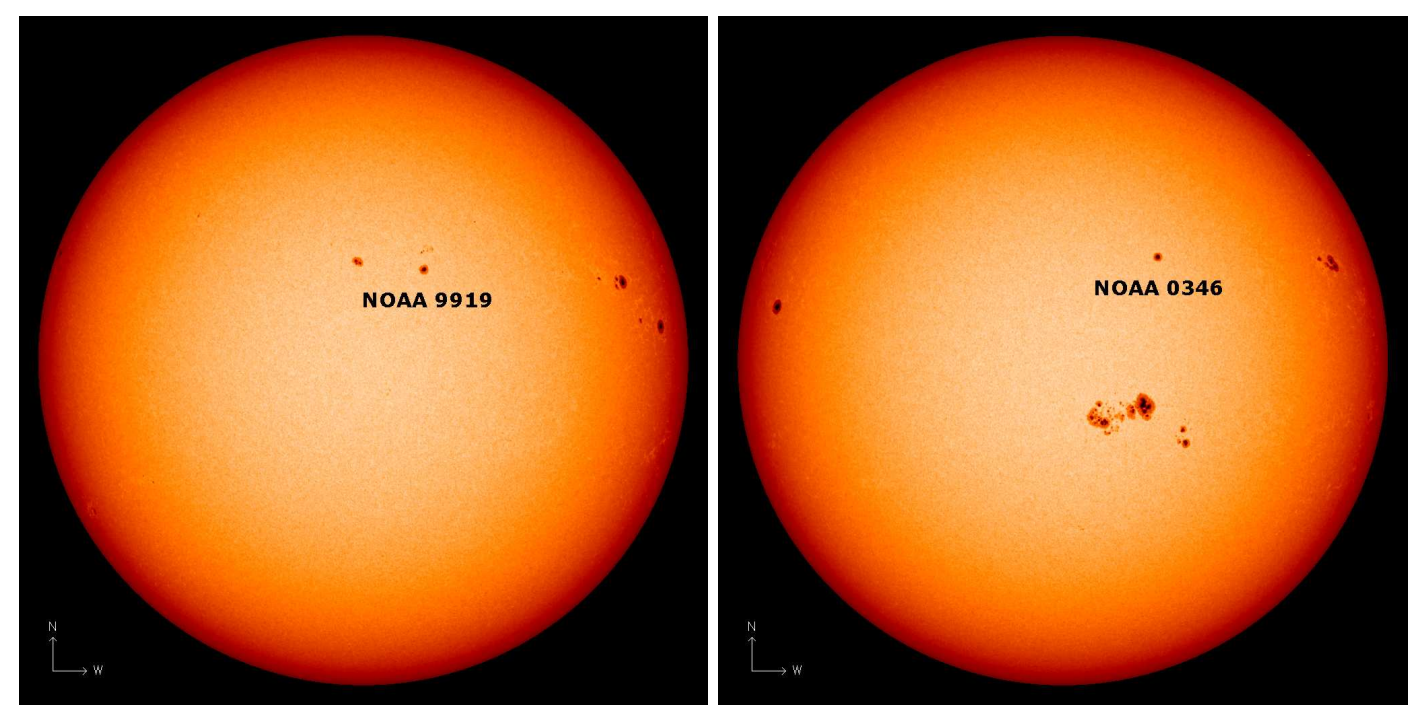

Figure 3.3: Continuum images with NOAA active region numbers showing the observed sunspots NOAA9919 (April 29, 2002), left, and NOAA0346 (May 2, 2003), right. From the Michelson Doppler Imager (MDI) on board of the Solar and Heliospheric Observatory (SoHO).

The size of the detectors $\left(384 \times 286\right.$ pixels) equivalent to $40^{\prime \prime} \times 30^{\prime \prime}$ on the solar surface limits the FOV. When using the polarimeter each $\frac{1}{2}(\mathrm{I}+\mathrm{V})$ and $\frac{1}{2}(\mathrm{I}-\mathrm{V})$ circularly polarised component takes half of the detector. Then the FOV, reduced by a factor of 2 , is not big enough for the observation of an entire sunspot of typical size at once. Therefore, we first observe one half of the sunspot and then the second one (Fig 3.4). Nowadays, these limitations have disappeared with the use of larger detectors installed in the described optical setup of the 'Göttingen' FPI.

\subsection{Data reduction and image restoration}

Next I describe the procedures to correct the images from the effects introduced by the instrumentation and by Earth's atmosphere. The treatment of the data has been performed

\footnotetext{
${ }^{2} \theta$ is used here, as is common in the literature, for the heliocentric angle or angle between LOS and vertical in the atmosphere. It must not be confused with the angle between LOS and the magnetic field, which is also commonly denoted by $\theta$.
} 

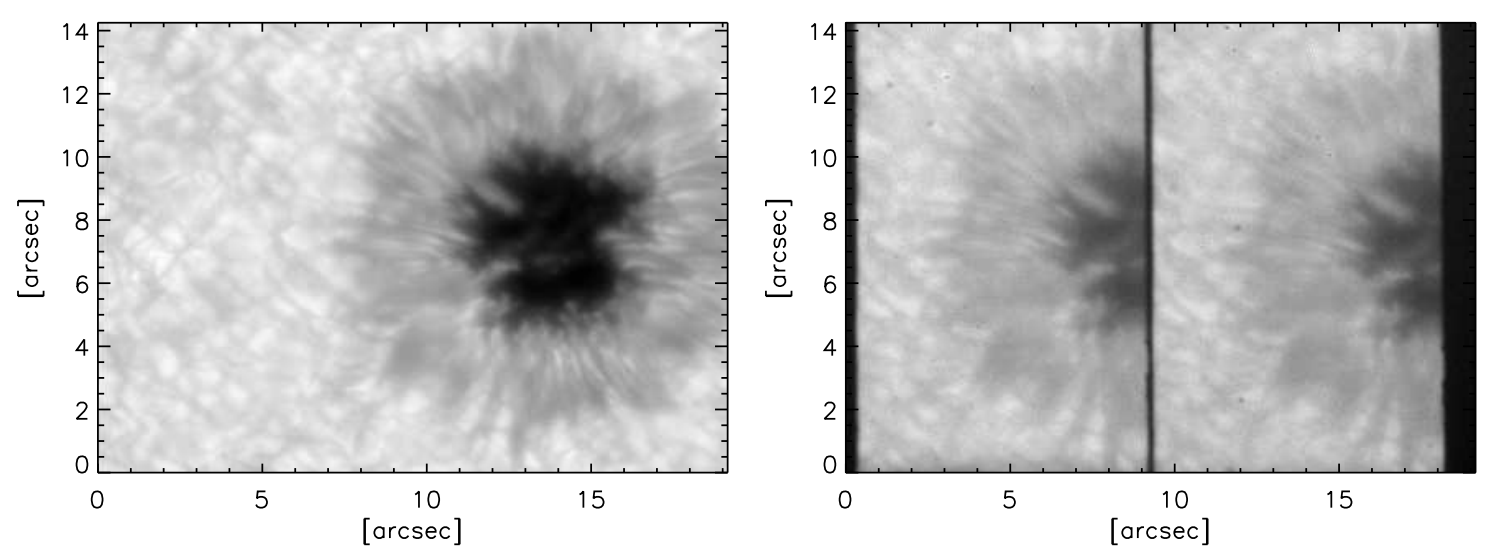

Figure 3.4: Examples of NOAA0346 raw data. Left: broad-band image. Right: narrow-band image in continuum showing both $1 / 2(I+V)$ and $1 / 2(I-V)$ polarimetric components.

using Interactive Data Language (IDL) programs and packages, many of them developed at the Universitäts-Sternwarte Göttingen.

\subsubsection{Broad-band channel}

The first step is to reduce and to restore the broad-band-channel data. We proceed as follows:

Dark correction. We subtract an offset of counts introduced by the read-out electronics and by thermal electrons in the CCD chip. For that we use an average 'dark' image obtained by taking a series of frames with the same exposure time as for the data frames but blocking the light path.

Flat fielding. It corrects for the different response of each pixel exposed to a homogeneous radiation. The 'flat field' images are exposures of solar light while moving the telescope pointing in 'random walk' manner near the Sun's disc centre region, trying to avoid solar structures such as sunspots. A large amount of these frames (always taken with the same characteristics in time and number of frames as the data images) will give a good average without any kind of solar structure such as the granulation pattern. This flat-fielding process will also correct for dust on the detectors and filters and other effects (interference fringes, ...) associated with the instrumentation. The common way to correct the data is then

$$
\text { corrected data }=\frac{\text { raw data }- \text { dark }}{\text { flatfield }- \text { dark }} .
$$

Speckle reconstruction. Ground-based observations are strongly affected by the turbulences in the terrestrial atmosphere, the seeing. The changes of the refractive index along the line of sight cause a variation of the light path through the atmosphere. As a result, a degraded object intensity is observed. With long exposure, the image is blurred. The information on structures at small scales is thus lost. To correct for such effects, many 
techniques have been developed in the last years. On the one hand, adaptive optics systems coupled to the telescopes can partly correct in real time the distorted wave fronts of the incoming light. On the other hand, image reconstruction techniques allow to correct for the degradation a posteriori as part of the data reduction. Both, the use of adaptive optics and reconstruction techniques are providing the high spatial resolution which is required for the research into the solar fine-structure.

The optical transfer function (OTF) is the Fourier transform of the point spread function which characterises the atmospheric seeing and the telescope diffraction and aberration effects. The solid angle on the sky in which the OTF does not vary is called isoplanatic patch. It has a diameter of $5^{\prime \prime}-8^{\prime \prime}$ in daytime seeing. Within an isoplanatic patch, the Fourier transform $I$ of the observed intensity distribution can be expressed

$$
I(\mathbf{q})=I_{0}(\mathbf{q}) S(\mathbf{q})
$$

$I, I_{0}$ and $S$ are functions of the spatial frequency vector in the Fourier domain, $\mathbf{q}=\mathbf{f} / f_{c}$, where $f_{c}=D / \lambda R$ is the cut-off frequency, calculated from the diameter of the (circular) entrance pupil $D$, the wavelength $\lambda$ of the light and the focal length of the telescope $R$.

The time scale $\tau_{a}$ of variation of the terrestrial atmosphere is short $(\leq 30 \mathrm{~ms})$, so that images with short exposure time, affected only by the instantaneous random OTF are taken. The average of a series of $N$ single exposures gives

$$
\sum_{i=1}^{N} I_{i}(\mathbf{q})=I_{0}(\mathbf{q}) \sum_{i=1}^{N} S_{i}(\mathbf{q}) .
$$

It would result in a long exposure image $\sum_{i} I_{i}(\mathbf{q})$ in the Fourier domain, where the information at high frequencies is lost by the cancellation in the complex Fourier components in $\sum_{i} S_{i}(\mathbf{q})$ with a cut-off at approximately $r_{0}^{2} / \lambda R$. Here, $r_{0}$ is the Fried parameter (Fried 1966). It may be interpreted as the diameter of a telescope with the same diffraction limit. For daytime seeing, its value is in the range of few $\mathrm{cm}$ to $50 \mathrm{~cm}$.

Labeyrie (1970) proposed to perform the average by summing the squared modulus of the Fourier components in order to avoid cancellations:

$$
\frac{1}{N} \sum_{i=1}^{N}\left|I_{i}(\mathbf{q})\right|^{2}=\left|I_{0}(\mathbf{q})\right|^{2} \frac{1}{N} \sum_{i=1}^{N}\left|S_{i}(\mathbf{q})\right|^{2} .
$$

Now, the speckle transfer function (STF), $\frac{1}{N} \sum_{i=1}^{N}\left|S_{i}(\mathbf{q})\right|^{2}$, preserves high-frequency components in the average and extends up to the telescope diffraction limit. The STF contains the Fried parameter $r_{0}$.

The basis of the speckle reconstruction technique used in this work and developed at the Universitäts-Sternwarte Göttingen is, working in the Fourier space, the separate restoration of the amplitudes and the phases of the image. The amplitudes are restored using the spectral ratio method proposed by von der Lühe (1984) and the phases through 
the speckle masking method initially introduced by Weigelt (1977) for astronomical observations.

Spectral ratio method. To avoid the use of the object transform $I_{0}$ which is the unknown, von der Lühe proposes to divide the squared modulus of the averaged observed Fourier transform by the average observed power spectrum,

$$
\epsilon(\mathbf{q})=\frac{|\langle I(\mathbf{q})\rangle|^{2}}{\left\langle|I(\mathbf{q})|^{2}\right\rangle}=\frac{\left|I_{0}(\mathbf{q})\right|^{2}}{\left|I_{0}(\mathbf{q})\right|^{2}} \frac{|\langle S(\mathbf{q})\rangle|^{2}}{\left\langle|S(\mathbf{q})|^{2}\right\rangle}
$$

which depends only on the telescope and seeing conditions. To evaluate $\epsilon$, theoretical expressions are used for the two averages, the STF (Korff 1973) and the average shortexposure transfer function $|S(\mathbf{q})|^{2}$ (Fried 1966, see also de Boer 1993, and Denker 1996). The behaviour expected for this ratio is a slow monotonic decrease for low frequencies and a steep decrease at $q \simeq r_{0} / D$. For given $\epsilon(q)$ the Fried parameter $r_{0}$ can be determined by comparison of the models of $\epsilon$, which contain $r_{0}$, and with the observed $\epsilon$. Then with $r_{0} / D$ known, the model STF can be evaluated and the amplitudes of $I_{0}$ can be retrieved according to Eq. 3.8. The number of images $\mathrm{N}$ taken should be large enough to ensure that arithmetic averages approximately equal ensemble averages. The larger $\mathrm{N}$ the better the approximation and the better will be the determination of $r_{0}$ from the quotient.

Speckle masking method. It consists in recovering the phases of the object transform $I_{0}$ from a quantity called bispectrum:

$$
B(\mathbf{q}, \mathbf{p}))=\langle I(\mathbf{q}) I(\mathbf{p}) I(-\mathbf{q}-\mathbf{p})\rangle .
$$

We may consider the image as an $L \times L$ matrix of pixel intensities. Then, the bispectrum can be written in discrete form

$$
B(i, j, k, l)=\langle I(i, j) I(k, l) I(-i-k \cdot-j-l)\rangle
$$

where averages are taken over all $\mathrm{N}$ speckle images. Equation 3.11 can be written

$$
B(i, j, k, l)=I_{0}(i, j) I_{0}(k, l) I_{0}(-i-k,-j-l) \times\langle S(i, j) S(k, l) S(-i-k .-j-l)\rangle .
$$

It can be shown that, upon sufficient averaging, the bispectrum of $S$ becomes a real function, i.e. its phases are zero. Thus, $B$ contains the phases of $I_{0}$ only. The essential speckle masking signal resides in a region where the spatial frequencies $\mathbf{q}, \mathbf{p}$ and $\mathbf{q}+\mathbf{p}$ exceed the seeing limit. Here, information on the object transform inaccessible to conventional techniques can be recovered. Using Euler's formulation for complex numbers we can equate the exponential factors in both terms through the closure equation

$$
e^{i \varphi(i+k, j+l)}=e^{i \varphi(i, k)} e^{i \varphi(j, l)} e^{-i \Phi(i, j, k, l)}
$$

where $\varphi$ is the object phase, i.e. the Fourier phase of the final reconstructed image and $\Phi$ is the bispectrum phase. The initial value to start the recursive calculation is $\varphi(0,0)=0$. Thus, step by step, the object phases are recovered. 
The reconstruction is applied to each subimage of the size of the isoplanatic patch, into which the images were divided. Afterwards, they are ressembled to recover the original size of the image. Fig 3.5 shows and example of the data before and after reconstruction. In the snap-shot or 'speckle' image from the original data, (a), the good quality of the data taken under good seeing conditions can be seen. In (b) the average over the whole series of 150 frames shows a smoothed image, analogue to a long exposure image. Finally, (c) shows the reconstructed image with a clear enhancement in contrast and good definition in the small details. The spatial resolution achieved comes close to the diffraction limit of the telescope, i.e. $0^{\prime \prime} .2$. Thus, the information of the small structures is recovered with high signal-to-noise ratio.

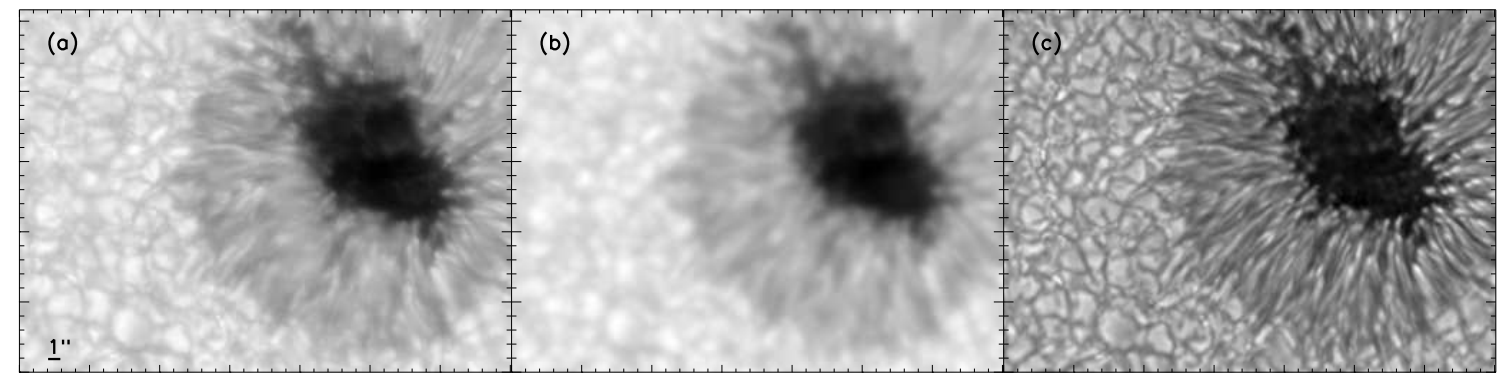

Figure 3.5: Broad-band reconstruction of NOAA 9919. Left: speckle image with short exposure time. Middle: average image from 150 speckle images. Right: speckle-reconstructed image.

Once the broad-band channel data have been reduced and reconstructed, we continue with the narrow-band ones. These images also need a careful treatment since the light path passes through several optical devices which introduce different effects to be taken into account.

\subsubsection{Narrow-band channel}

Continuum correction. Taking images from a homogeneous source of white light (halogen lamp) it is possible to measure and correct the effect that the transmission curve of the interference filter (IF2 in Fig. 3.1) introduces into the spectral line profiles under observation.

Narrow-band reconstruction. Since the narrow-band (NB) images contain spectral information, they possess highly valuable information about the dynamics of solar structures. The broad-band data set provides also interesting information about the structure of the deep layers in the solar photosphere. Yet, an additional important purpose why they are taken simultaneously while scanning with the FPI is to perform the reconstruction of the spectroscopic data as is explained next.

The speckle reconstruction method described above demands a large number $(\sim 100)$ of images for reasons of the statistical properties of Earth's atmosphere. The method cannot be applied to the NB images from each wavelength position where only 4-8 images 
can be taken. The reconstruction method, proposed by Keller \& von der Lühe (1992), and implemented, e.g. by Janssen (2003), is based on the fact that data taken simultaneously suffer from the same atmospheric effects. Thus, we can write in the Fourier domain

$$
\begin{aligned}
& I_{\mathrm{BB}, i}(\mathbf{q})=I_{0, \mathrm{BB}}(\mathbf{q}) S_{i}(\mathbf{q}) \\
& I_{\mathrm{NB}, i}(\mathbf{q})=I_{0, \mathrm{NB}}(\mathbf{q}) S_{i}(\mathbf{q})
\end{aligned}
$$

where every single exposure, $I_{\mathrm{BB}, i}$ and $I_{\mathrm{NB}, i}$, i.e. the Fourier transforms of every observed intensity distribution in $\mathrm{BB}$ and $\mathrm{NB}$ are the result of the same effect of the instantaneous optical transfer function $S_{i}$ on the object transforms, $I_{0, \mathrm{BB}}$ and $I_{0, \mathrm{NB}}$. The above speckle reconstruction gives an estimate of the true broad-band scenery $I_{0, B B}$ on the Sun.

We use the error metric for the narrow-band images

$$
E=\sum_{i}\left|I_{\mathrm{NB}, i}-I_{0, \mathrm{NB}} S_{i}\right|^{2}
$$

Replacing $S_{i}$ by means of Eq. 3.16 and minimising $E$ yields

$$
I_{0, \mathrm{NB}}(\mathbf{q})=H \frac{\sum_{i} I_{\mathrm{NB}, i} S_{i}^{*}}{\sum_{i}\left|I_{\mathrm{BB}, i}\right|^{2}} I_{0, \mathrm{BB}}(\mathbf{q})
$$

where an optimum filter $H$ is included to reduce noise. To construct $H$ the level of noise is estimated from defocused flat-field images which, taken under identical observational conditions as the data, do not show solar structures.

The procedure of restoration is applied to both circularly polarised components $\frac{1}{2}(\mathrm{I}+\mathrm{V})$ and $\frac{1}{2}(\mathrm{I}-\mathrm{V})$.

Blueshift correction. Our FPIs are mounted in the collimated parallel beam close to an image of the telescope pupil. This introduces changes in the wavelength of maximum of transmission of the FPI across the field of view. The wavelength shift depends on the angle of incidence $(\Theta)$ on the FPIs through (from a second order expansion of Eq. 3.2)

$$
\Delta \lambda \approx-\lambda_{0} \frac{\Theta^{2}}{2}
$$

where $\lambda_{0}$ is the wavelength of the maximum of transmission at normal incidence. The wavelength displacement visible in the line profiles as a shift relative to the scanning position (and apparent redshift to higher wavelength values) has been quantified across the whole field of view from the flat-field images which should not show any kind of shift related to solar structures. Then, it is only needed to apply the correction in displacement to the data set for each spatial position (pixel). More details of this effect and its correction can be found in the thesis works of Koschinsky (2001) and Janssen (2003).

Alignment of $\frac{1}{2}(\mathbf{I}+\mathbf{V})$ and $\frac{1}{2}(\mathbf{I}-\mathbf{V})$. The intensity and the magnetic information of the data will be provided by the analysis of the Stokes $I$ and Stokes $V$ parameters obtained from the addition and subtraction, respectively, of both circularly polarised light components: 


$$
\begin{aligned}
I & =1 / 2(I+V)+1 / 2(I-V) \\
V & =1 / 2(I+V)-1 / 2(I-V)
\end{aligned}
$$

Thus, a precise alignment (to sub-pixel accuracy) in spatial direction between the components has been done to make sure that no false signal was introduced when adding or subtracting.

Second order flat-fielding. We calculate the Stokes $V$ signal from the flat-field images in use. We expect it to be zero since these images are observed avoiding solar structures and a large amount of them has been used to get a proper average. However, a small signal of the order of $0.04 \%$ of the continuum intensity remains due to small errors in the flat fields (gain tables) and to a residual wavelength shift in one polarised light channel with respect to the other. Thus, a second order flat-fielding correction has been applied as explained in the thesis by Socas Navarro (1999), following the expression

$$
V^{\text {corr }}=V^{1}-\frac{V_{\text {flat }}}{I_{\text {flat }}} I^{1}
$$

where $V^{1}$ and $I^{1}$ are the Stokes data profiles after first order flat-fielding and the correction factor $\frac{V_{\text {flat }}}{I_{\text {flat }}}$ (Fig. 3.6) is calculated from the Stokes signals of the average flat field.

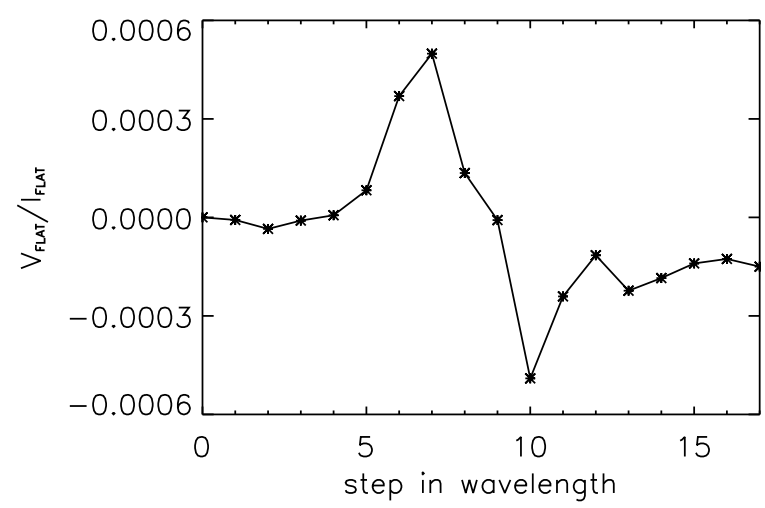

Figure 3.6: Example of correction factor used in the second order flat-fielding obtained from the Stokes $V$ and $I$ of the average flat field.

\subsubsection{Deconvolution with a PSF}

Tests have shown that the speckle-reconstructed broad-band images become nearly identical with the narrow-band continuum images when the former are convolved with a Gaussian of FWHM of $0{ }^{\prime \prime} 4$. The reason for the reduced spatial resolution of the narrow-band 
images comes, presumably, from additional imaging through the filtergraph (via lenses, folding mirrors, interference filters, polarimeter). The broad-band images possess a spatial resolution certainly better than $0^{\prime \prime} 3$. A spatial resolution of the narrow-band continuum images of better than $\left(0.3^{2}+0.4^{2}\right)^{1 / 2}$ arcsec $=0.5$ follows.

To further improve the resolution, we deconvolved the narrow-band images at $6302 \AA$ with a Gaussian point spread function of 0'.35 FWHM, applying a Tikhonov regularisation. The width of $0 ! 35$ was chosen conservatively to keep noise at a low level. This yields a spatial resolution of the narrow-band images better than 0'.35.

\subsubsection{Instrumental polarisation}

The study based on polarised light requires a detailed analysis of the instrumental polarisation or crosstalk introduced by retardation upon oblique reflections of the incoming light in the optical system. The observed Stokes vector $\mathbf{I}^{\prime}$ in terms of the incoming, solar I is given by

$$
\mathbf{I}^{\prime}=\mathrm{MI}
$$

where $\mathbf{M}$ is the Mueller matrix describing the polarisation properties of the optical system. In terms of the elements of the matrix

$$
\left(\begin{array}{l}
I^{\prime} \\
Q^{\prime} \\
U^{\prime} \\
V^{\prime}
\end{array}\right)=\left(\begin{array}{llll}
M_{11} & M_{12} & M_{13} & M_{14} \\
M_{21} & M_{22} & M_{23} & M_{24} \\
M_{31} & M_{32} & M_{33} & M_{34} \\
M_{41} & M_{42} & M_{34} & M_{44}
\end{array}\right)\left(\begin{array}{c}
I \\
Q \\
U \\
V
\end{array}\right) .
$$

The instrumental crosstalk of the VTT has been measured early in the nineties by Hofmann (2000) and later by Collados (1999). Also recently Beck et al. (2005) have presented a polarisation model for the VTT. With the values of the Mueller matrix of the telescope from these papers some estimates have been made. This work is focused on circularly-polarised-light data, therefore, only the analysis of the crosstalk introduced in the Stokes $I$ and $V$ signals is needed. For the $6302 \AA$ line pair we estimated the influence of the instrumental polarisation on the measurements in the following way: We calculated Stokes profiles emergent from the penumbra model by del Toro Iniesta et al. (1994) applying reasonable values of magnetic fields, of their inclination and gas velocities. From these profiles and the relevant elements of the instrumental Mueller matrix of the VTT the effects of the crosstalk on the measurements were calculated.

The small amplitudes of the polarisation signal compared to the intensity give approximately $1 \%$ of $Q, U, V \rightarrow I$ crosstalk which gives only negligible influence on the velocity measurements. For the measured Stokes $V$ ' signal we obtain

$$
V^{\prime}=M_{41} I+M_{42} Q+M_{43} U+M_{44} V .
$$

The contribution from $I \rightarrow V$ is small as can be seen from Fig. 3.6. Besides, this effect has been taken into account during second order flat fielding. The main concern comes from the $Q, U \rightarrow V$ crosstalk, i.e. from the elements $M_{42}$ and $M_{43}$, which are of the order of 0.3 , while the $Q$ and $U$ amplitudes, are of the same order of 0.1 (relative to 
the continuum intensity $I_{\mathrm{c}}$ ) as the $V$ amplitudes. In the upper panels of Fig 3.7 three examples of the amplitudes of synthetic Stokes profiles for the spectral lines at $\lambda 6302 \AA$ are shown. They have been calculated from the mean penumbral atmosphere model by del Toro Iniesta et al. (1994), for different angles (between the magnetic field direction and the observer). In the lower panels the Stokes $V$ signals unaffected $(V)$ and affected $\left(V^{\prime}\right)$ by crosstalk are represented. The $V$ amplitude of Fe I $6301.5 \AA$ is changed by crosstalk by approximately $10 \%$ in average. This will be the error of the effective magnetic field estimates when applying the Weak Field Approximation (Chapter 47). The values of the effective magnetic field calculated from the centre of gravity method (COG) of the Fe I $6302.5 \AA$ profiles, described in the next chapter, will be underestimated by $20 \%$, at most, because of crosstalk, while the distance of the $V$ extrema of this line is not affected.
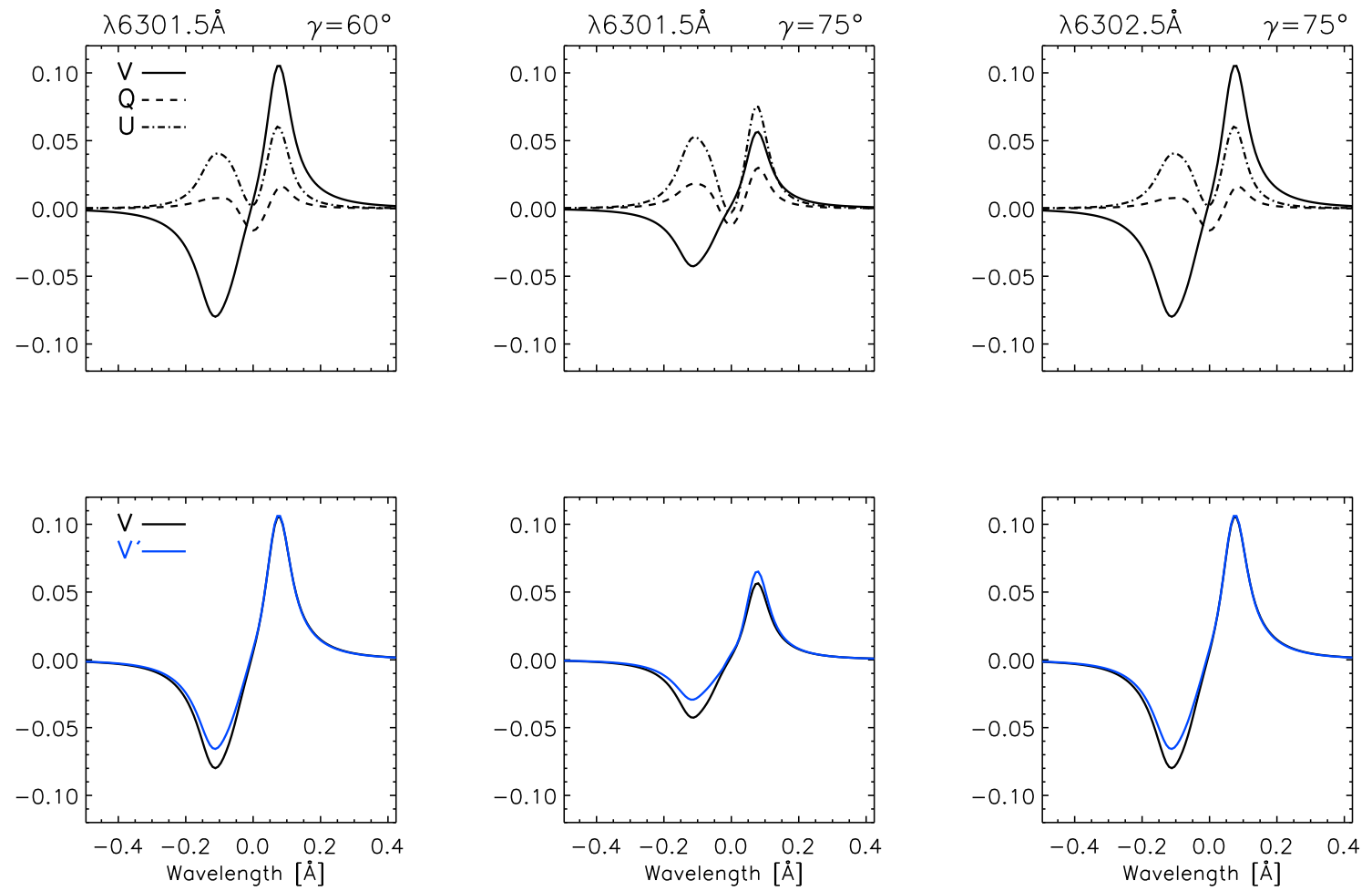

Figure 3.7: Examples of the effect of the instrumental crosstalk on Stokes profiles. Upper panels: Stokes $Q, U$ and $V$ signals at different inclination angles for the line pair at $6302 \AA$. Lower panels: Stokes $V$ signal pure $(V)$ and affected by crosstalk $\left(V^{\prime}\right)$.

\section{Fe II $6149.3 \AA$ : line with no instrumental polarisation}

The Fe II 6149.3 A line, among others (see Vela Villahoz et al. 1994), has a special behaviour that makes it interesting for the present study: It is insensitive to instrumental polarisation. The ionised iron line is formed through the transition ${ }^{4} D_{1 / 2} \rightarrow{ }^{4} P_{1 / 2}$. The total angular momentum of the lower and the upper levels of the transition are then $J_{l}=1 / 2$ and $J_{u}=1 / 2$ and the Landé factors are $g_{l}=0$ and $g_{u}=4 / 3$, respectively. Therefore, if 
we calculate the displacements of the $\pi$ and $\sigma$ components through Eq.2.7 we find that they coincide in wavelength. Moreover, the $\sigma$ components have twice the strength of the $\pi$ components and hence it follows for the absorption and dispersion profiles that

$$
\phi_{p}=\frac{\phi_{b}+\phi_{r}}{2}, \quad \psi_{p}=\frac{\psi_{b}+\psi_{r}}{2} .
$$

This means by Eqs.2.39 and 2.42 that $\eta_{Q}=\eta_{U}=\rho_{Q}=\rho_{U}=0$. Finally, it follows from the RTE that $Q=U=0$. The result is a spectral line which shows no linear polarisation of solar atmospheric origin. Thus, no instrumental crosstalk $Q, U \rightarrow V$ can occur.

The absence of information about the linear polarisation, i.e. of the orientation of the magnetic field, could be a disadvantage, but in this work the study has been concentrated in the observed circular polarisation signal.

\subsubsection{Scattered light}

The photons coming from the Sun suffer scattering by the dust particles in the terrestrial atmosphere and also in their path through the telescope. Mainly the scattered light from the quiet Sun photosphere introduces some false light in sunspots and may contaminate the calculations of the velocity field. For the Fe II $6149.3 \AA$ line, the contribution of the scattered light has been estimated from its umbral profile. In a 'cool' atmosphere like a sunspot umbra the formation of this ionised iron line is not expected. Therefore, the observed profile is assumed to be pure scattered light. It is then possible to account for the correction needed to remove this effect from the profiles in the penumbra. For the $6302 \AA$ lines this effect is estimated by assuming that the false light reaches a reasonable level of $10 \%$ of the continuum intensity in the penumbra and that it carries an un-shifted line profile from the quiet Sun. Simulations have shown that, from the influence of this spurious light, the line shift measurements, without accounting for the scattered light, turn out to be underestimated by $10 \%$. 


\section{Penumbral fine-structure: data analysis}

The dynamics and magnetism of sunspot penumbrae appear to be housed in the finestructure. Spectroscopic data with high spatial resolution as those described in the previous chapter are therefore needed to extract the information contained at such small scales. The present chapter is devoted to the analysis of the observational data. In the first section, the intensity fluctuations of the penumbral features will be treated. Then, the magnetic field calculations by means of polarimetric data will be described. Next, the Evershed flow will be studied applying different methods to estimate the velocity fields. The last section will treat the relation and possible correlation between the three different parameters - intensity, magnetic fields and velocities - characteristic of the penumbral fine-structure (cf. Bello González et al. 2005).

\subsection{Intensity maps}

From the data, two different kinds of intensity maps can be presented, maps from broadband images, which originate essentially from continuum forming atmospheric layers, and maps corresponding to different wavelength positions from the narrow-band images, i.e. from different heights in the sunspot atmosphere. From the broad-band images a first analysis of the penumbral structure can be done. Fig. 4.1 represents examples of such images for both NOAA 0346 and NOAA 9919 sunspots, both at a heliocentric angle of $\theta \sim 20^{\circ}$. One can notice the higher definition of the image for NOAA 9919 compared to the one for NOAA 0346. The difference stems from the different seeing conditions during observations.

The intensity maps from the narrow-band channel are obtained by adding the twodimensional circularly polarised components after the reduction and proper treatment of the data described in previous sections. These maps will be called below filtergrams, since they are obtained by observation with narrow filters.

\section{Differentiating centre- and limb-side penumbra}

Let us first emphasise the importance of the differentiation of the penumbra in centreand limb-side penumbra. From broad-band intensity maps, such as in Fig.4.1, one can notice that penumbral grains, i.e. penumbral bright points, appear brighter in one side of the sunspot than in the other. Also, the fact that the Evershed effect is characterised by 

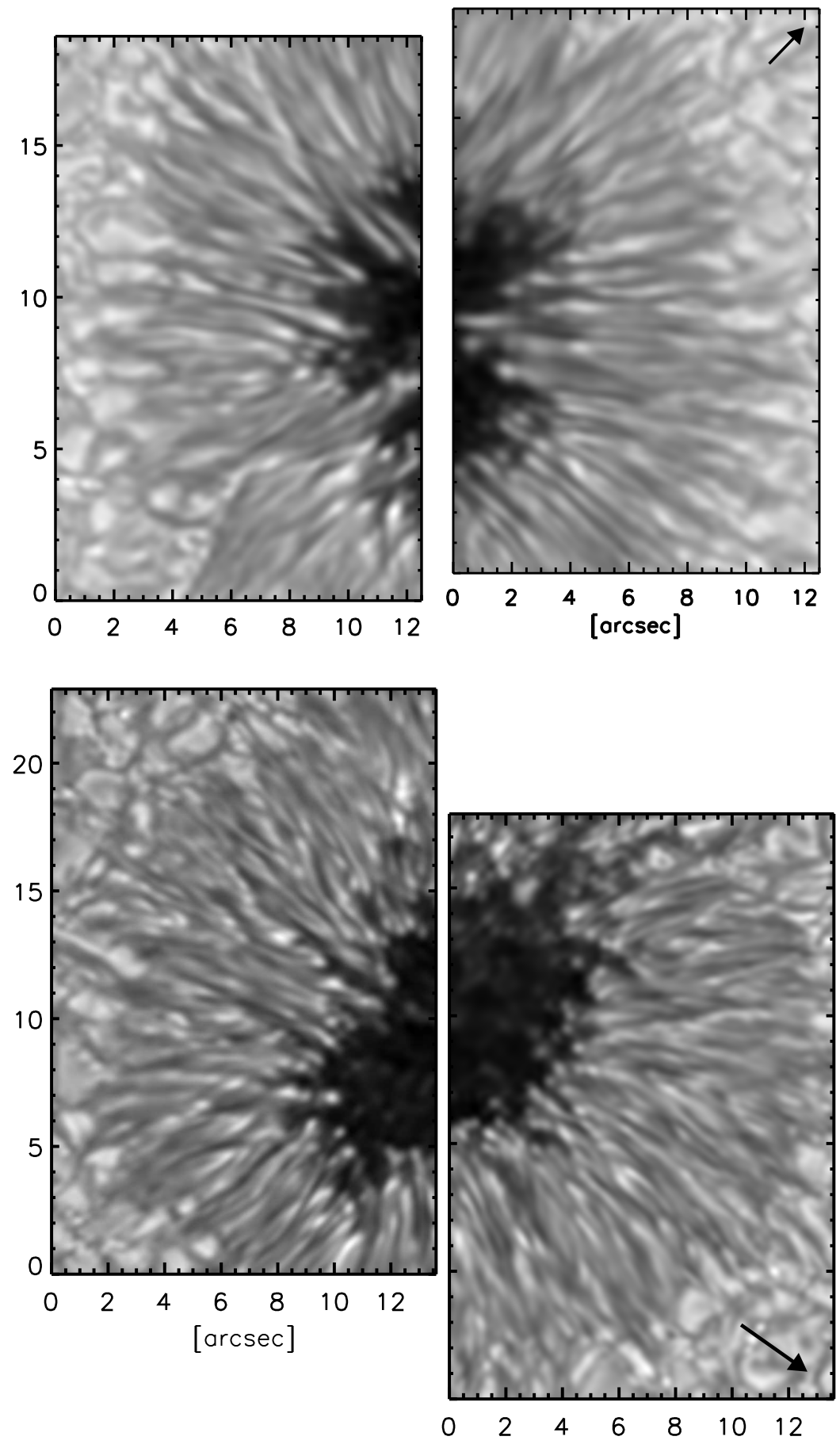

Figure 4.1: Examples of intensity maps of sunspots, NOAA 0346 (upper panels) and NOAA 9919 (lower panels), from broad-band images. Arrows point towards disc centre.

blue-shifted and red-shifted spectral lines, i.e. flow velocities towards and away from the observer, respectively, indicates the need for such a differentiation.

Since the Sun is a sphere, the imaging of the solar surface structures implies projection effects. The heliocentric angle accounts for the position of a solar structure with respect 
to the disc centre and therefore, it accounts also for the projection effects. For sunspots, the centre-side penumbra will refer to the region of the sunspot which is closer to the disc centre and the limb-side penumbra to the region closer to the solar limb.

\subsubsection{Photometric properties of penumbrae}

\section{Penumbra in broad-band images}

A penumbra, as seen in broad-band images, is mainly characterised by its bright and dark alternating filamentary structure laying along the radial direction of the sunspot. When, for a given radius, an azimuthal cut is considered, the intensity variations look like it is shown in Fig.4.2 It is difficult to say whether the bright filaments lie on a darker background or vice versa.
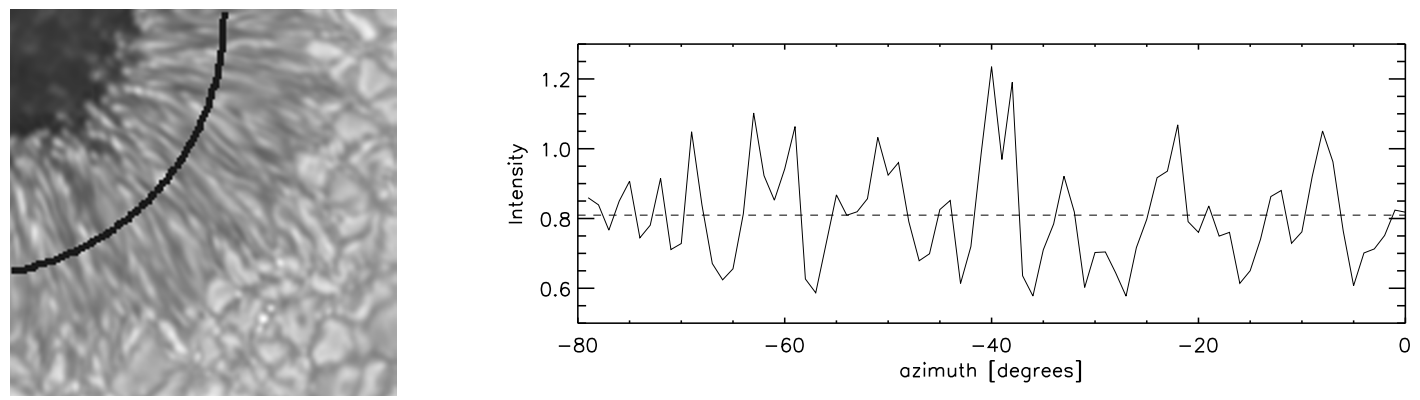

Figure 4.2: Azimuthal cut of NOAA 9919 center-side penumbra. Left: region of the spot where a ring of given radius crossing the mid penumbra is marked in black. Right: Intensity variation along the azimuthal cut. Intensity values refer to the mean intensity value of the surrounding quiet photosphere. The dashed line gives the mean intensity along the cut.

The heads of the bright filaments or penumbral grains show intensity values above the mean continuum intensity $I_{c}$ of the ambient photosphere and, therefore, comparable to the intensity of bright granules. These very bright structures appear more frequently in the limb-side than in the centre-side penumbra, which was already noticed by Tritschler et al. (2004) and Sütterlin et al. (2004). The tails of such filaments, apparently attached to the bright heads, have an average intensity of $0.7 I_{c}$ with some achieving values of $0.9 I_{c}$.

\section{Radial dependence of penumbral intensities}

Before discussing in detail the small-scale properties of penumbrae we point out, differentially for centre and limb side, some global properties that depend on the radial distance from spot centre. Such global variations should certainly also be explained by viable models of penumbra dynamics.

Taking advantage of the high symmetry of the sunspots NOAA 0346 and NOAA 9919, it is also interesting to study the intensity variation along the radial direction. Moreover, by using the various filtergrams, i.e. intensity maps taken at different wavelengths within the different spectral lines, such a study can be extended to compare the radial dependence of the intensity originating from different layers of the sunspot atmosphere. The procedure consists in measuring the average intensity along the azimuthal direction of the sunspot 
for a given radial position. We have performed the averaging at many radial positions by 'scanning' from the surrounding photosphere until the dark umbra within the field of view. This procedure is appplied separately for both, the centre-side and the limb-side filtergrams. For NOAA 9919, in order to avoid effects in the average intensity values from 'irregular' structures in the penumbra, only the penumbral area shown in Fig. 4.2 has been taken into account, for the centre-side averaging.

The result are curves of average intensity at various wavelengths within spectral lines along the radial distances. Some examples are shown in Fig.4.3. There, curves for the limb and the centre side are shown separately in the left and right panels, respectively.

In all cases the general behaviour is the expected increase of the intensity from the umbra to the photosphere. The minimum mean intensity value for the umbra of NOAA 9919 varies from 0.2 to 0.4 relative to the mean continuum intensity in the surrounding photosphere for the various filtergrams along the Fe I line pair. In the case of NOAA 0346 the umbra average minimum intensity value is of the order of 0.4 . No changes in this value for different wavelengths occur since the Fe II line is not expected to form in the 'cool' umbral atmosphere. The above relative intensity values are not corrected for spurious light originating from scattering in the Earth's atmosphere and in the telescope. Thus, they should not be taken cum grano salis.

From the curves presented in Fig.4.3, we notice in both sunspots a non-monotonic behaviour of the intensity within the penumbral boundaries:

- In NOAA 9919 this phenomenon is better seen in the limb-side intensities (left panels) showing similar behaviour in the two neutral iron lines. The minimum of intensity occurs at 0.85 normalised radial distance from the centre of the spot. The phenomenon is observed in intensity curves from filtergrams at all - line core, line wing and continuum - wavelengths. However, it is better seen in the line core of $\lambda 6302.5 \AA$ than in the line core of $\lambda 6301.5 \AA$. This may be due to the different heights of formation of the two lines. As will be seen in the next section, the line core of $\lambda 6301.5 \AA$ forms in higher layers than that of $\lambda 6302.5 \AA$. This would mean that this decrease in intensity occurs less pronounced in intensities from higher layers of the penumbral atmosphere. The stronger enhancement of the intensity in the core of $\lambda 6302.5 \AA$ in the limb side could also be interpreted by the Zeeman splitting and weak $\pi$-component of this line, due to a strong longitudinal component of the magnetic field. Very likely, this interpretation is inadequate since such a strong intensity enhancement is not observed in the centre side of the sunspot.

- In the centre side, the average intensity values oscillate. Such oscillations may result from the statistically low numbers of bright and dark structures along the azimuth at fixed radial distance. Yet we emphasise that they are present at all wavelengths. Therefore, the phenomenon occurs at atmospheric layers where the Fe I lines are formed, which indicates that this phenomenon covers a large range of atmospheric heights.

- In NOAA 0346 , observed in $\lambda 6149.3 \AA$, the brightness peak in the penumbra appears closer to the umbral boundary in the limb-side penumbra than in the centre- 

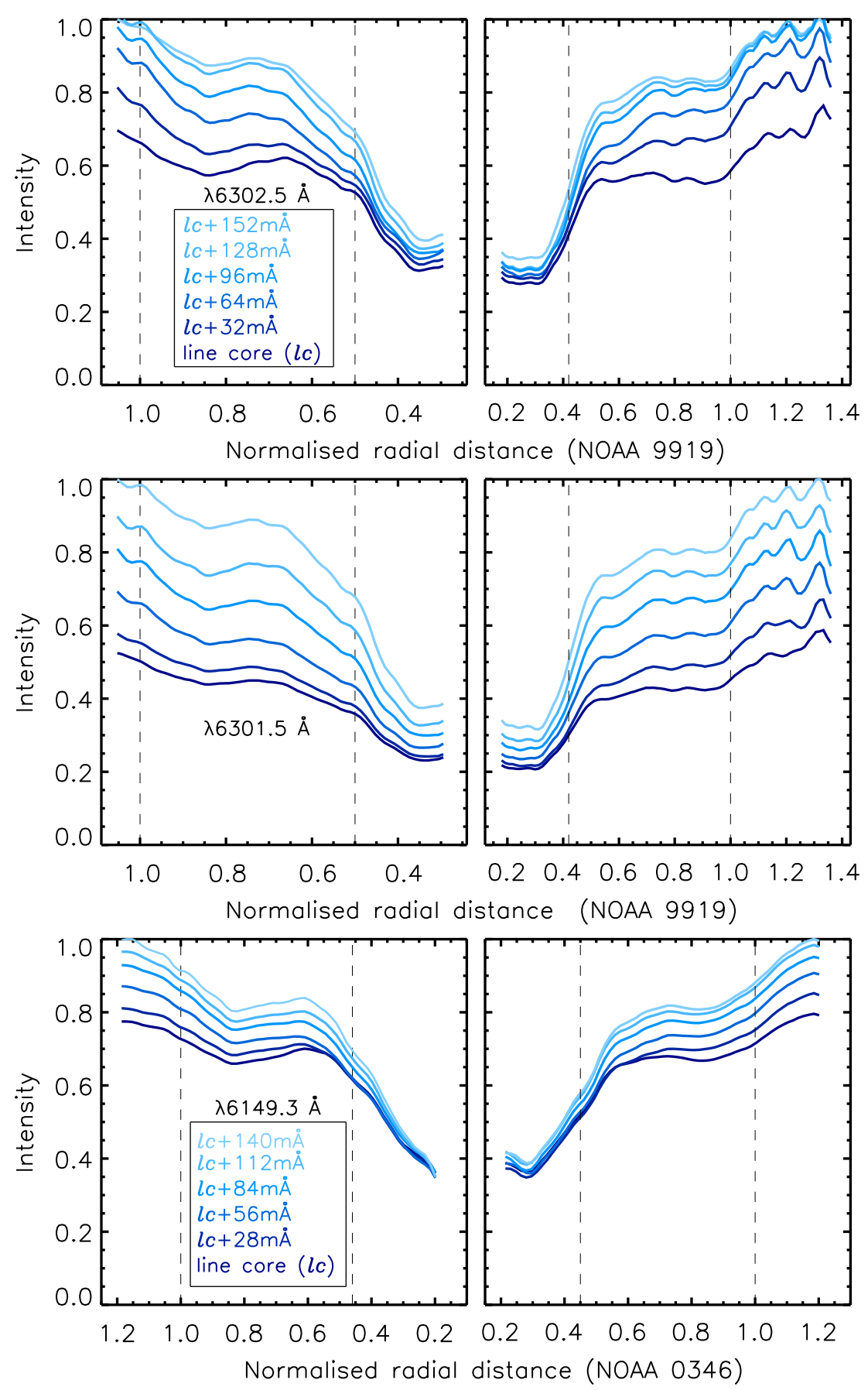

Figure 4.3: Curves of mean intensity along the radial direction. Top: mean intensity along radial distance from sunspot centre for various filtergrams at wavelengths close to the line centre of $\lambda 6302.5 \AA$ (given in the box) for limb side (left) and centre side (right) of the sunspot NOAA 9919. Middle: same as top panels but for wavelengths around $\lambda 6301.5 \AA$. Bottom: same as top panels but for indicated wavelengths around $\lambda 6149.3 \AA$ for NOAA 0346. Abscissas are in units of normalised radial distance to the centre of the spot and the vertical lines denote the penumbral boundaries. 
side penumbra. It is also seen that the effect of enhancement is more pronounced in the curves corresponding to line centre filtergrams.

This brightness enhancement, also observed by Tritschler et al. (2004) and Westendorp Plaza et al. (2001a), is still not well understood. Differences between limb side and centre side may be due to the presence of bright penumbral grains carrying hot material from deeper layers to the photosphere and which are better seen in the limb-side penumbra. Thus, along such an interpretation, thermal and opacity effects varying with distance from the spot centre would underlie the intensity variations.

If we compare intensity curves along the radial distance of filtergrams from the blue and the red wings of a given spectral line some additional differences are found. They are depicted in Fig.4.4. When both curves follow a similar behaviour but with different intensity values we can interpret this by 'global' blueshifting or redshifting motions. Blueshifts, as those characterising the centre-side penumbra, produce an enhancement of intensity in the red wing and a decrese of intensity in the blue wing. Likewise, redshifts (as those in the limb-side penumbra) produce an enhancement of intensity in the blue wing and a decrease in the red wing.
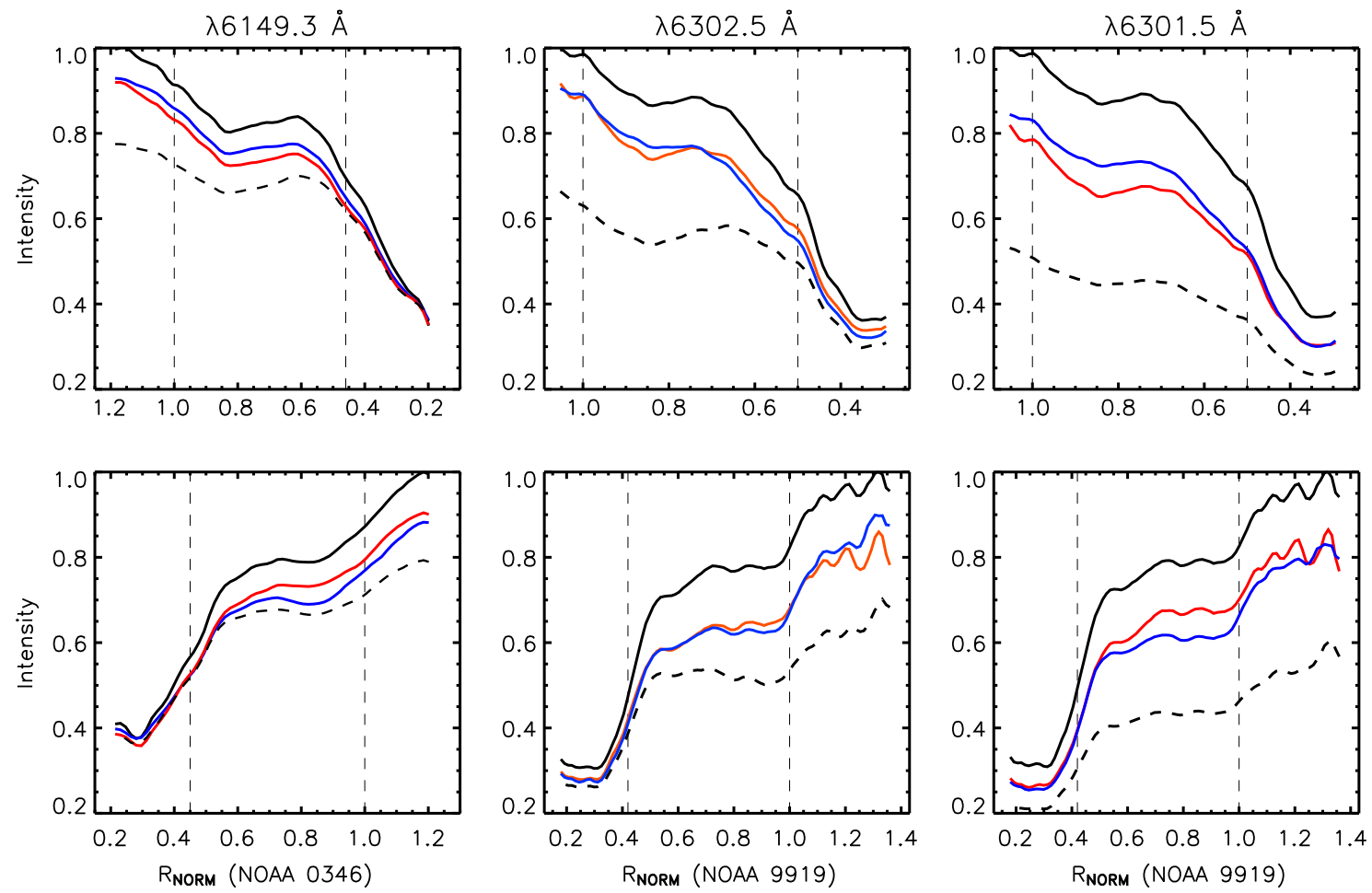

Figure 4.4: Radial dependence of average intensity in sunspots for different wavelenghts. Top: Curves of average intensity in the limb side of the sunspots. Bottom: Curves of average intensity in the centre side. Solid lines represent curves at wavelengths close to continuum. Dashed lines represent curves at wavelengths close to the line centre. Red and blue lines represent curves from the red and blue wings of the spectral lines, respectively. The abcissas give values of normalised radial distances from the centre of the sunspot. Vertical lines denote the inner and the outer penumbral boundaries. 


\subsubsection{Spectroscopic properties of penumbral intensity}

Let us now make use of the spectroscopic information from the observational data through the Stokes I (or intensity) profiles to infer some more general properties about the sunspot atmosphere and more specifically about the penumbral atmosphere. As we know, Stokes $I$ profiles give us information along the spectral direction and, therefore, along the different layers of the atmosphere where such lines are formed.
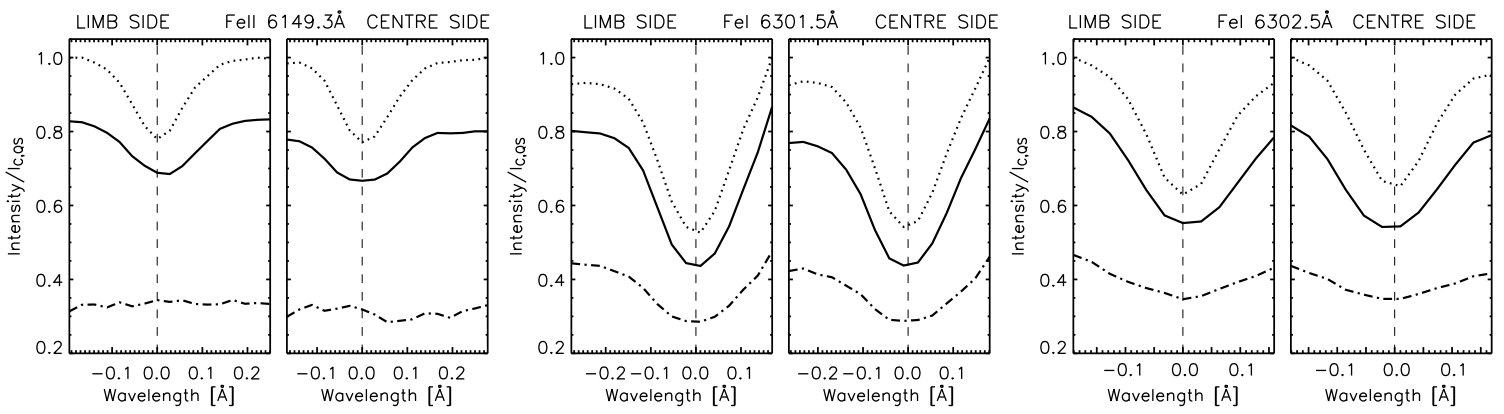

Figure 4.5: Intensity (Stokes I) profiles for the Fe I 6302 A pair (in sunspot NOAA 9919) and the Fe II 6149.3 $\AA$ (sunspot NOAA 0346) spectral lines. Solid lines show mean penumbral intensity profiles along the spectral lines. Dotted lines show the mean intensity of sourrounding quiet-Sun photosphere, $I_{\mathrm{QS}}$. Zero position on the wavelength axis, denoted by the vertical dashed line, is set to line centre of $I_{\mathrm{QS}}$. Intensities are normalised to $I_{\mathrm{QS}}$ continuum values.

In Fig.4.5 mean intensity profiles for umbra, penumbra and surrounding quiet-Sun photosphere are shown for NOAA 9919 observed in the line pair at $\lambda 6302 \AA$ and for NOAA 0346 observed in the ionised iron line $\lambda 6149.3 \AA$, in representations for the limb and the centre side. The intensity profiles are normalised to the continuum of the quietSun photosphere $I_{\mathrm{c}, \mathrm{QS}}$. Let us summarise the main properties of the intensity profiles:

- penumbral profiles (solid lines) exhibit in the continuum about $0.8 I_{\mathrm{c}, \mathrm{QS}}$, while umbral profiles (dashed-dotted lines) show $\sim 0.45 I_{\mathrm{c}, \mathrm{QS}}$. As mentioned before, these values are not corrected for scattered light;

- the Fe II $6149.3 \AA$ line is absent in the cool umbra;

- for all three lines, the mean intensity profiles from the limb-side penumbra are displaced towards higher wavelength values (redshifted) with respect to the mean 'reference' $I_{\mathrm{c}, \mathrm{QS}}$ profile;

- the mean intensity profiles from the centre-side penumbra are displaced towards lower wavelength values (blueshifted) with respect to the mean 'reference' $I_{\mathrm{c}, \mathrm{QS}}$ profile.

These wavelength displacements of the mean intensity profile across the whole penumbral area are a first indication of the strong dynamics characteristic of penumbrae.

Normalisation of the profiles to their local continuum leads us to other interesting properties shown in Fig.4.6. 
- penumbral intensity profiles (as well as umbral profiles) appear broader than the $I_{\mathrm{c}, \mathrm{QS}}$ profiles. The broadening may be caused by both, magnetic (Zeeman) and thermal motion (Doppler broadening) effects;

- in the Fe II 6149.3 $\AA$ line, intensity profiles show weaker line depression than $I_{\mathrm{c}, \mathrm{QS}}$. This is an indication of the lower degree of ionisation in the cooler penumbra compared to the surrounding hotter photosphere. For the same reason, Fe I $6301.5 \AA$ line profiles show stronger line depression in the penumbra;

- however, the line depression shown by Fe I $6302.5 \AA$, which stems from the same multiplet as $\lambda 6301.5$, is comparable to that of the surrounding photosphere profiles. This line undergoes a strong Zeeman broadening $(g=2.5)$ which reduces the line depression.
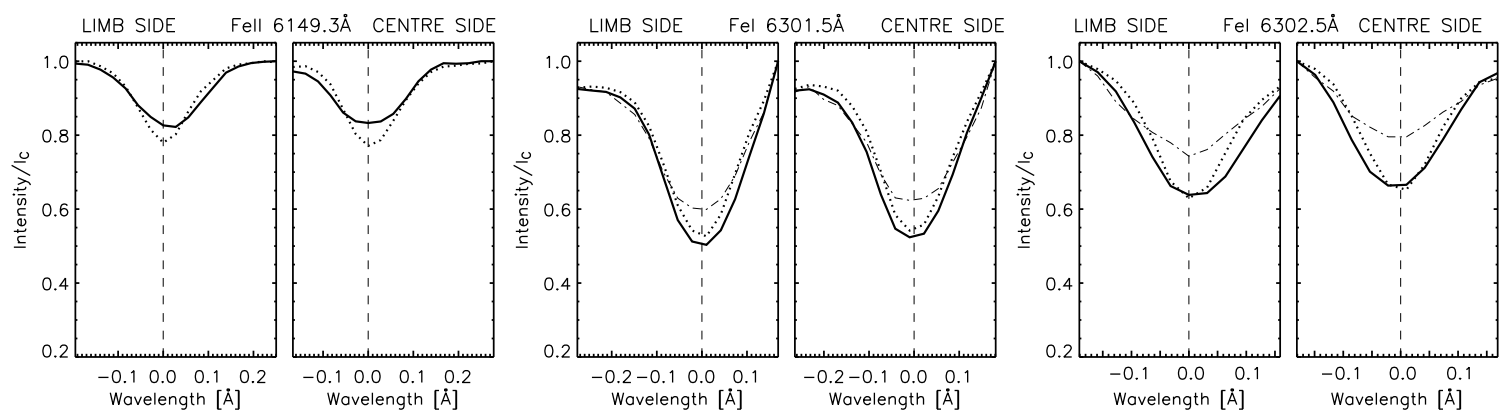

Figure 4.6: Same as in Fig.4.5 but intensity profiles normalized to the local continuum.

The umbral line profiles are not reliable. The observations were not meant to study sunspot umbrae. So, the counts in the umbral profiles were low, thus the noise is high, and the spectral scans were too short to reach true continuum near split lines.

\subsubsection{Temporal evolution}

Apart of the evolution with time of the surrounding granulation, the inward movement of the radial penumbral filaments, near the umbra-penumbra border, can be already observed within an image time sequence of about $8 \mathrm{~min}$ ( 10 consecutive scans with time cadence of $50 \mathrm{sec}$ ). The migration of the penumbral grains into the umbra are the most clear evidence of such dynamics in this time interval and it has been even possible to estimate an average motion speed of $0.6 \mathrm{~km} \mathrm{~s}^{-1}$ by tracing the distance covered within such time, in agreement with measurements by Sobotka et al. (1999) and Sobotka \& Sütterlin (2001). An example of radial motions is shown in Fig.4.7 where an enlarged subfield of the inner limb-side penumbra of NOAA 3046 can be seen. There, $(A)$ and $(B)$ represent the best images of the sequence with a time difference of 5 min. Every structure appears displaced in $(B)$ with respect to $(A)$ by an inward motion. 


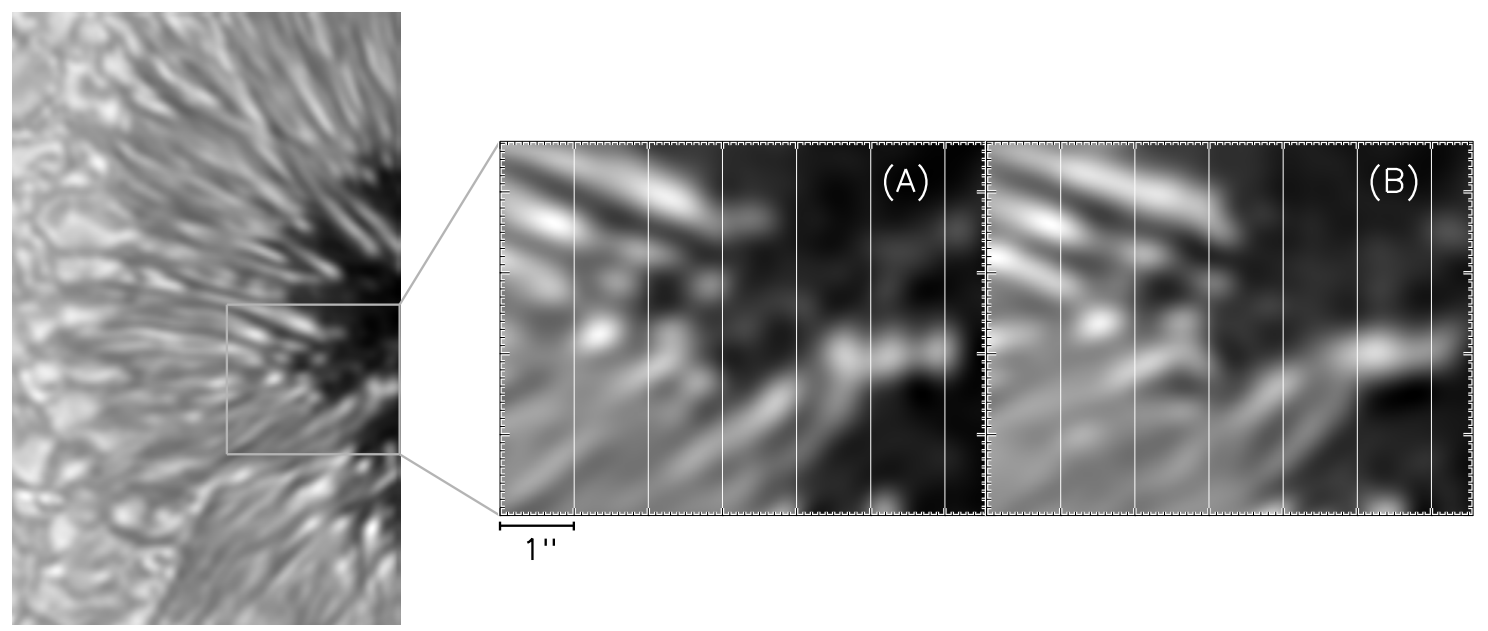

Figure 4.7: Example of radial motions of penumbral filaments close to the umbra border. Left: Continuum image of the limb side of NOAA 0346. Right: enlargement of the marked region. Time difference between $(A)$ and $(B)$ is 5 min. Distance between white lines is $1^{\prime \prime}$.

\subsubsection{Intensity fluctuations}

The intensity structure of the sunspot NOAA 9919 at a heliocentric angle of $\theta=20^{\circ}$ is shown in Fig.4.10 (upper panel). To suppress noise, for the image from the outer wings of the $6301.5 \AA$ line an average of frames at line centre $(l c)+256 \mathrm{~m} \AA$ and $l c-224 \mathrm{~m} \AA$ is taken. Images from pure continuum are not available in the data. Yet, for short, this latter image is denoted as 'continuum' image. Likewise, the line centre image is actually an average of the three images taken closest to $l c$.

\section{Temperature response functions}

To answer the question from which height in the atmosphere the measurements come, we have to know which layers of the atmosphere are contributing to the formation of the spectral lines in use or simply the height of formation of the given lines.

One possibility is to calculate response functions (Mein 1971; see also Krieg et al. 1999). Response functions ( $R F$ ) describe the effect that perturbations of a given physical parameter characteristic of the medium have on the emergent line intensity. Following Eibe et al. (2001), if $R F_{p}$ is the response function for a certain parameter $p$, the intensity fluctuation generated by a small perturbation $\Delta p$ can be written as

$$
\Delta I(\lambda)=\int_{0}^{+\infty} R F_{p}(\lambda, z) \Delta p(z) d z
$$

where $z$ represents the height variable within the atmosphere. Hence, $R F$ s are usually determined along a given spectral range (or spectral line) and they depend on the atmospheric height.

Here, intensity fluctuations are adopted as proxies for temperature fluctuations and, therefore, the interest is centred in temperature RFs. The average penumbral-atmosphere 
model by del Toro Iniesta et al. (1994) is used to obtain the average intensity profiles, $I_{a v}$. Then small temperature increases $\Delta T$, at specific heights $z_{i}$, are applied. Temperature response functions, $R F_{T}$ can then be calculated as

$$
R F_{T}\left(\lambda, z_{i}\right)=\frac{\delta I_{i}(\lambda)}{\frac{\Delta T}{T} I_{c} \Delta z},
$$

where $\delta I_{i}$ are the intensity fluctuations for a small perturbation $\Delta T$ affecting a depth interval $\left[\mathrm{z}_{i}, \mathrm{z}_{i}+\Delta z\right]$. In the actual calculations, $\delta I_{i}$ has been obtained from

$$
\delta I_{i}(\lambda)=\left(I_{i+1}(\lambda)-I_{a v}\right)-\left(I_{i}(\lambda)-I_{a v}\right)=I_{i+1}(\lambda)-I_{i}(\lambda) .
$$

$I_{i}$ and $I_{i+1}$ are obtained from $\Delta T$ perturbations at heights $z_{k} \geq z_{i}$ and $z_{k} \geq z_{i+1}$, respectively.

The response functions were convolved in wavelength with the transmission function of the spectrometer (see Sec.3.1) to account for its influence on the observed profiles. Calculations of the temperature $R F$ s have been performed for the three spectral lines of interest. Figure 4.8 shows examples of curves for five different wavelength positions and for three different magnetic field strengths $B$. The solid lines represent $R F_{T}$ at wavelengths in the continuum close to the spectral lines, the red coloured ones represent contribution functions from the line centre and the other three represent values at wavelengths within the wings: line centre $+40 \mathrm{~m} \AA$, line centre $+80 \mathrm{~m} \AA$ and line centre $+120 \mathrm{~m} \AA$. As expected, different spectral lines as well as different atmospheric conditions (variations in $B$ in this case) show different response to temperature perturbations within the atmosphere.

Intensity fluctuations in the line centres at $\lambda 6302.5 \AA$ and $\lambda 6149.3 \AA$ occur quite low in the photosphere, at about $100-200 \mathrm{~km}$ for the specific magnetic field configuration with constant field strength which was chosen. This tells us that the observed intensity fluctuations in the maps of the line centres correspond to layers not far above those layers from which the continuum originates $\left(\tau_{5}=1\right.$ or $z=0 \mathrm{~km}, \tau_{5}$ is the continuum optical depth at $5000 \AA$ ). However, $R F_{T}$ for the line centre at $\lambda 6301.5 \AA$ occurs heigher above the continuum forming layers.

Surprisingly, $R F_{\mathrm{T}}$ for $\lambda 6302.5 \AA$ at line centre has its maximum at lower heigths than $R F_{\mathrm{T}}$ in the wings of this line. The effect is also seen, but less pronounced, in the $\lambda 6149.3 \AA$ line. The solution of this paradox comes from the strong Zeeman splitting which lowers the height of formation at the line centre.

\section{Temperature response functions for the $\mathrm{Fe}$ I $6301.5 \AA$ line}

Figure 4.9 depicts temperature response functions $R F_{\mathrm{T}}$ for several wavelength in the Fe I $6301.5 \AA$ line. For typical penumbral field strengths within the range of $1000-2200 \mathrm{G}$ and field inclinations the response functions for wavelengths near the centre of this line are similar. Therefore only one set of $R F_{T}$ is shown.

The line core fluctuations are formed in the height range of $150-400 \mathrm{~km}$ (above $\tau_{5}=1$ ). There exists a small overlap with the response functions for the wings of the line ('continuum' image). The temperature response functions near the line centre look different 


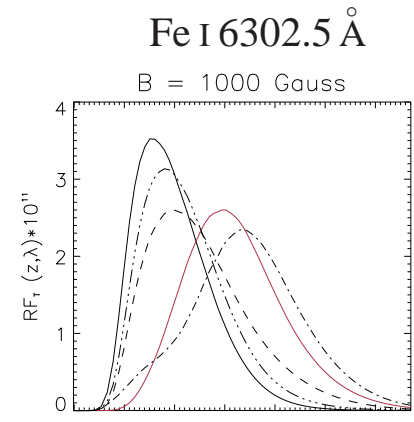

Fe I $6301.5 \AA$

$\mathrm{B}=1000$ Gauss
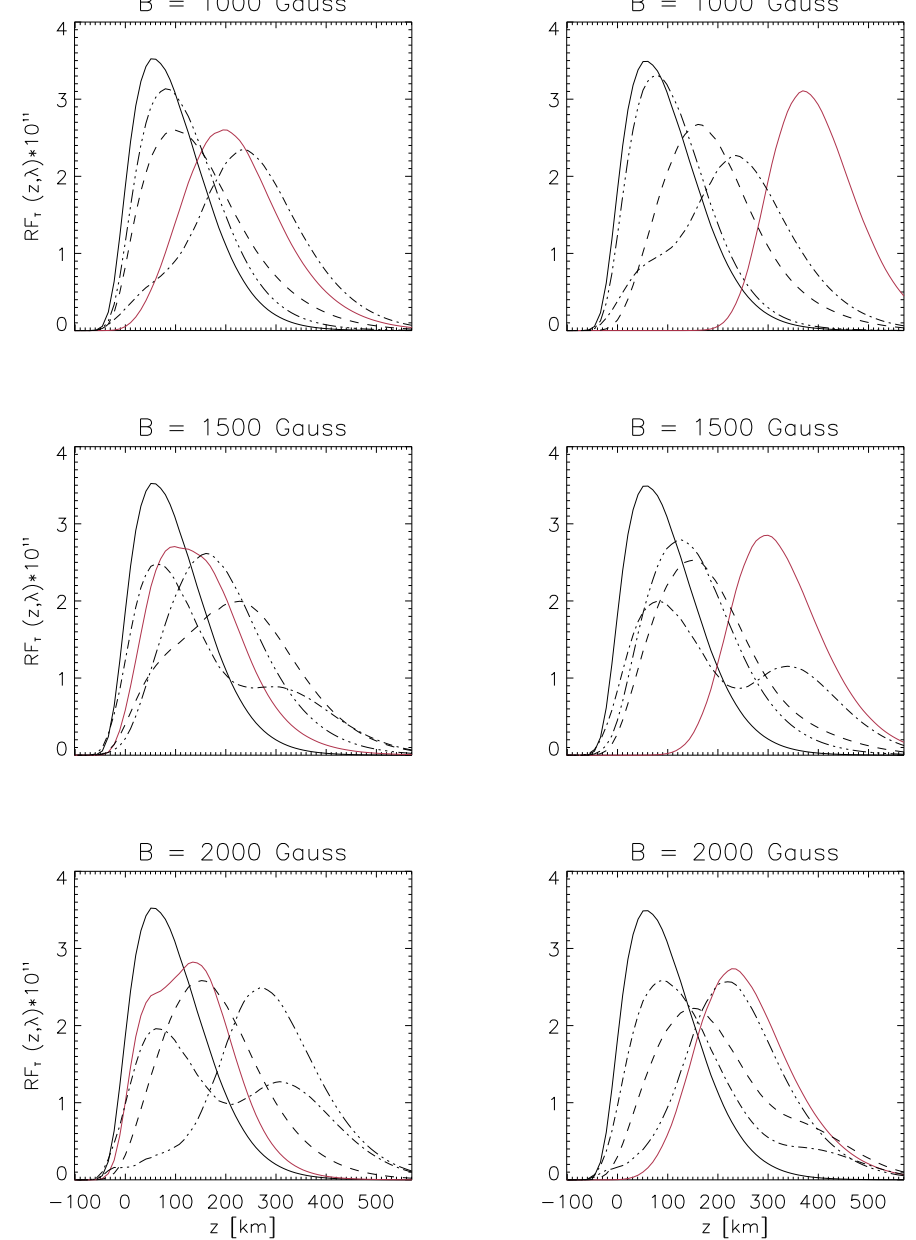

$B=1500$ Gauss
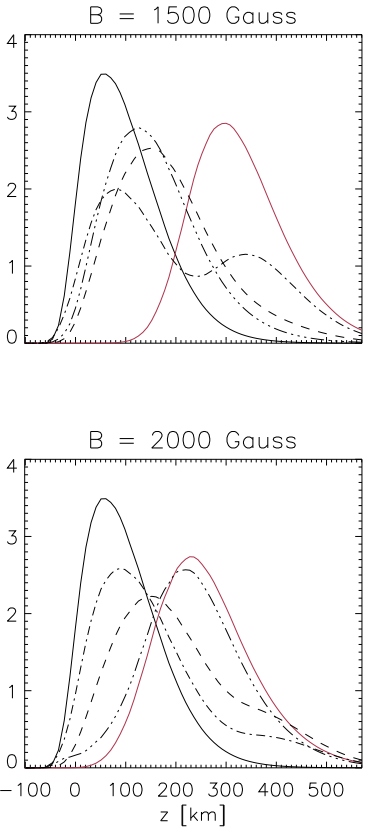

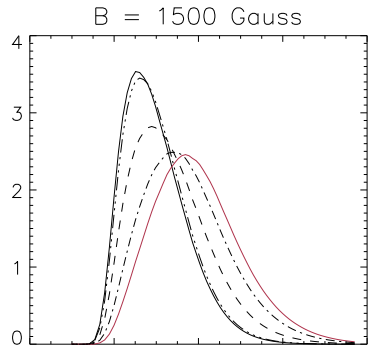

Fe II $6149.3 \AA$

$B=1000$ Gauss

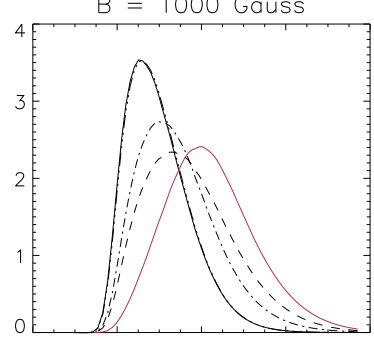

$B=1500$ Gauss

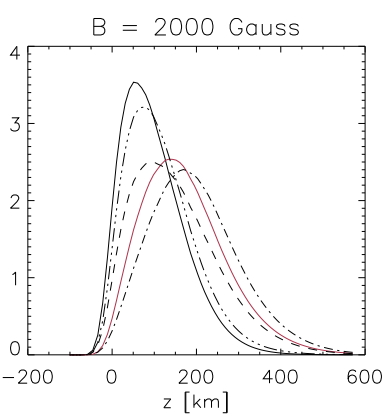

Figure 4.8: Examples of $R F_{T}$ for each of the three spectral lines. Solid curve represents $R F_{T}$ in the continuum near the line; red at the line centre wavelength; dashed at $40 \mathrm{~m} \AA$ from the line centre; dashed-dotted at $80 \mathrm{~m} \AA$ from the line centre and dashed-dot-dot at $120 \mathrm{~m} \AA$ from the line centre. All calculations were performed for $\gamma=45^{\circ}$.

for strong magnetic fields $(>2000 \mathrm{G}$ ) oriented closely along the LOS. Then the Zeeman splitting becomes strong and the light at wavelengths around the line centre comes from deep layers of the atmosphere. Thus, the response functions in Fig.4.9 are not designed for features in an umbra like, e.g., umbral dots, observed close to disc centre and with nearly vertical magnetic fields.

\section{Small-scale intensity fluctuations}

Since the origin of the intensity fluctuations, as proxies of temperature fluctuations, is known, one may study the variation of the penumbral structuring with height. Figure 4.10 shows intensity maps of the sunspot NOAA 9919 at continuum level and line centre of 


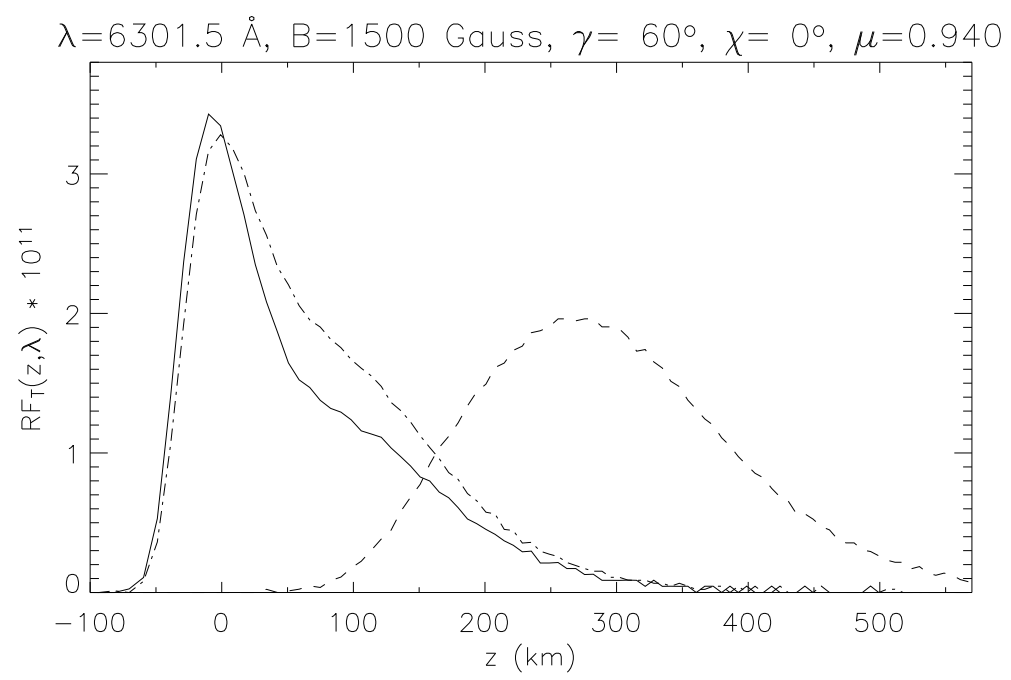

Figure 4.9: Temperature response functions for Fe I $6301.5 \AA$ in the penumbral model of del Toro Iniesta et al. (1994). The units of the response functions are $\mathrm{erg} \mathrm{cm}^{-2} \mathrm{~s}^{-1} \mathrm{~Hz}^{-1} \mathrm{ster}^{-1} \mathrm{~K}^{-1} \mathrm{~km}^{-1}$. Solid curve: continuum response function $R F_{T}$; dash-dotted: average from wavelengths in the outer wings; dashed: average from wavelengths around line center. $\gamma$ is the angle between the magnetic field and the LOS, $\chi$ the azimuth of the magnetic field and $\mu=\cos \theta$ ( $\theta$ is the heliocentric angle).

Fe I $6301.5 \AA$. The penumbral small-scale fluctuations in the continuum and line centre images are of the same order, 20-30\% peak to valley. Besides, they show a close correspondence, nearly one to one. At scales of $0.45-0$ '.5 and larger, the penumbral structures at deep photospheric levels are persistent up to heights of 300-400 km. This agrees with the results obtained from inversions of filtergram data in the Fe I $5576 \AA$ line (del Toro Iniesta et al. 1994) and of Ca II K spectrograms (Rouppe van der Voort 2002).

One notices that at scales larger than 0.5 the structures, or conglomerates of structures, retain their identity through the atmospheric part covered by the observations. Yet, very importantly, when moving from low to high layers, penumbral fibrils with diameters of 0.4 and less tend to change position, orientation, length and brightness relative to other ambient fine structures. Close inspection of Fig.4.10 shows that some of the very fine structures are visible only in one of the two images originating from two different atmospheric heights. At scales below 0!'4 the penumbral intensity fine structure changes with height in the atmosphere.

Examples of changes in intensity at small scales are shown in Fig.4.11 The upper two panels to the right represent the enlargement of the marked subfield of a region in the limbside penumbra of NOAA 9919 for both, continuum and line centre intensitites. The small faint structure close to the bright filament changes its position in one image with respect to the other. The lower panels show the same representation but for a subfield from the centre-side penumbra. The relative enhancemen 1 of the bright structure is clearly seen in the line core image. Similarly to these two examples, many other differences in the fine-structure can be found in the whole penumbral area.

\footnotetext{
${ }^{1}$ Note that each intensity subfield is scaled to its local mean intensity.
} 

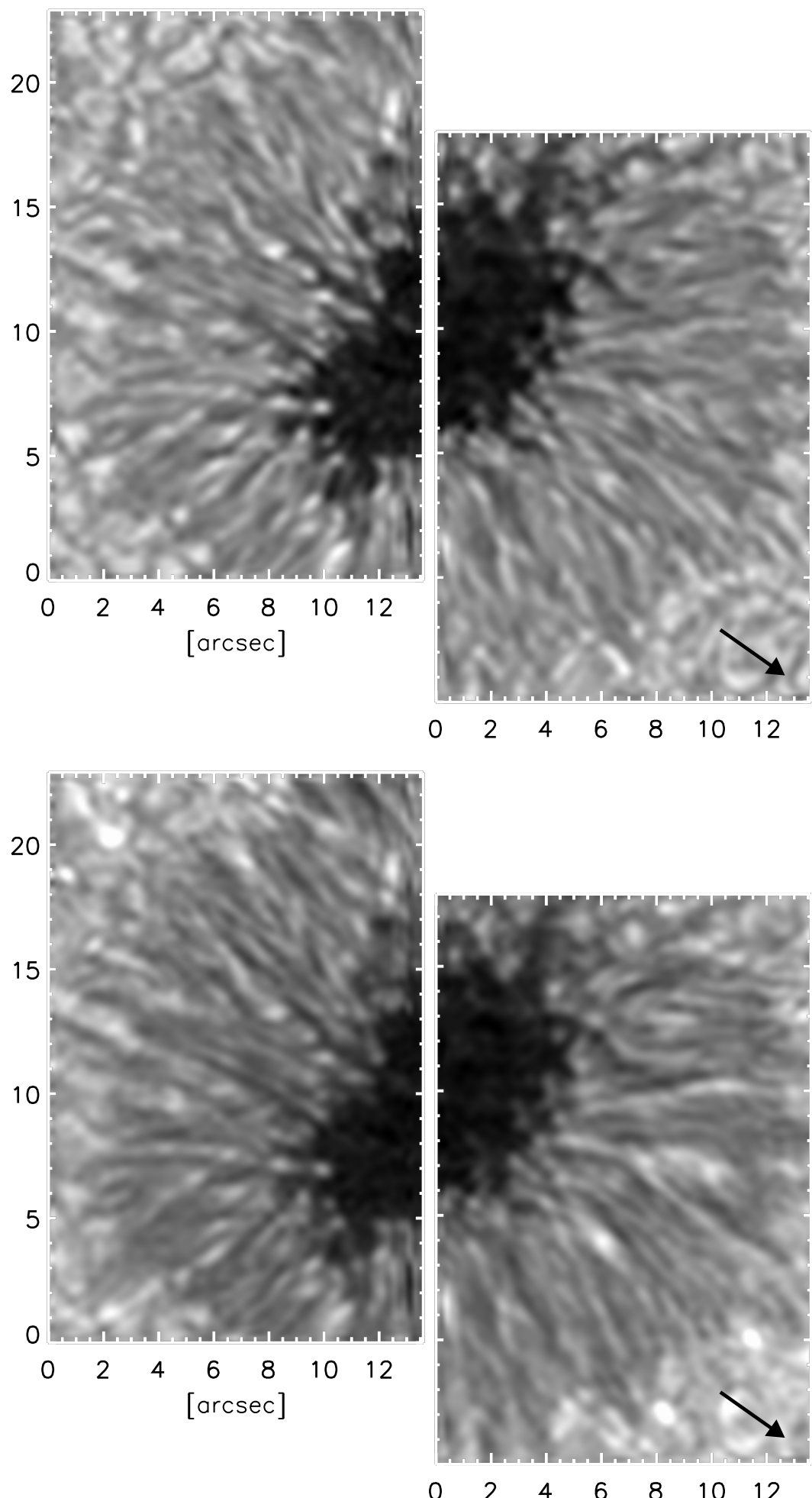

Figure 4.10: Examples of intensity maps of NOAA 9919. Upper panel: at continuum level. Lower panel: at line centre of $\lambda 6301.5 \AA$ (height of formation $300-400 \mathrm{~km}$ above $\tau_{5}=1$ in penumbral atmosphere). Arrows point towards disc centre. 

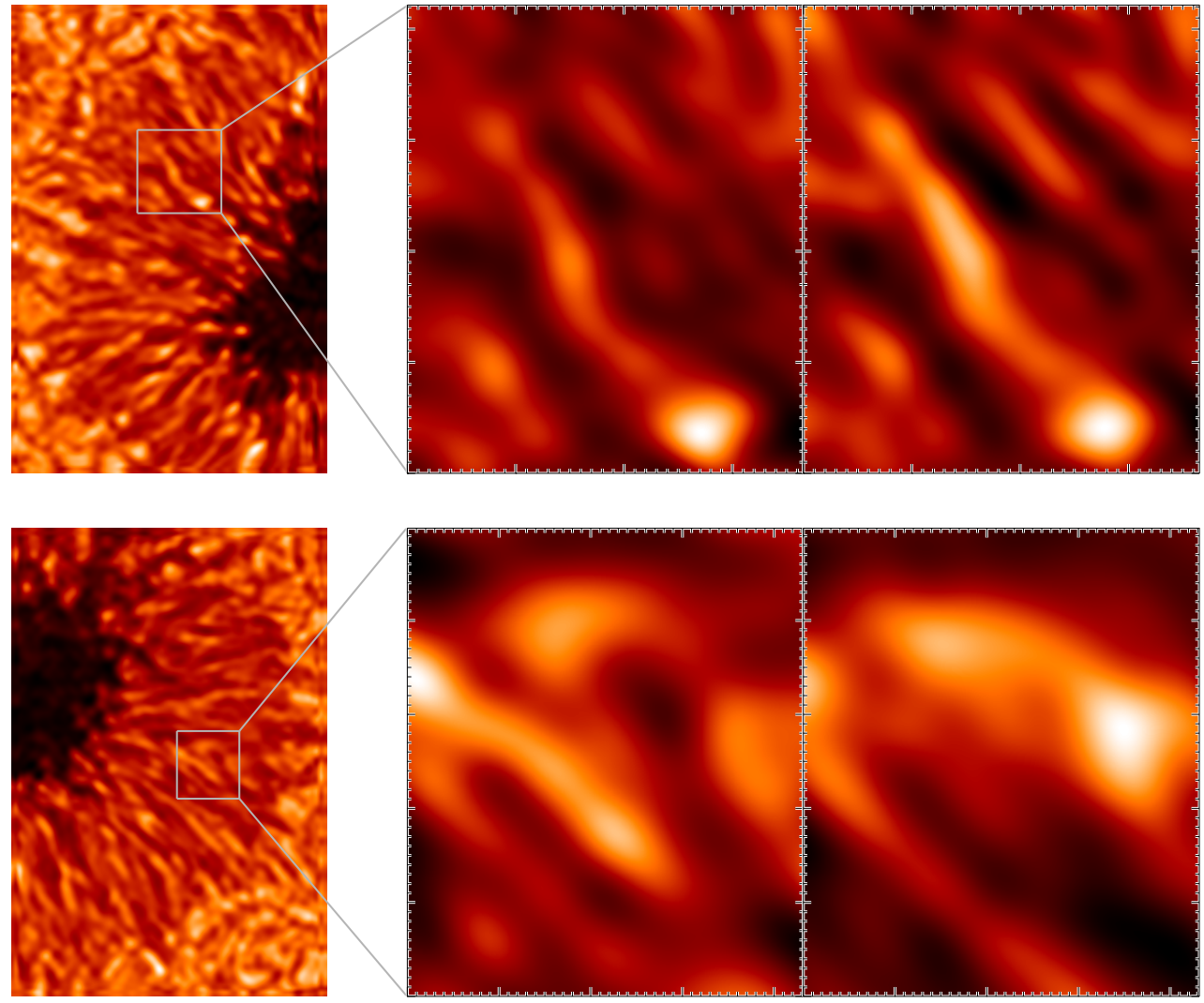

Figure 4.11: Details limb side (top) and centre side (bottom) of NOAA9919 in the $\lambda 6301.5 \AA$. Left: 'continuum' intensity map. Right: subimages of the same region at continuum and line centre wavelengths, respectively. Small tickmarks are at $0{ }^{\prime} 1$ distance.

\subsection{Magnetograms}

In this section the different procedures to calculate the magnetic field maps are described. All procedures are based on the physical fundament of the Zeeman effect and the analysis of the state of polarisation arising from the presence of magnetic fields. Such polarisation phenomena can be measured from observations to obtain the information about the orientation and strength of the magnetic fields.

\section{From Weak Field Approximation}

The calculation of the effective magnetic fields $B_{\text {eff }}$ from the WFA in Fe II $6149.3 \AA$ is based on the amplitudes of the extrema of the $V$ lobes as described in Sec.2.4.4. The algorithm conceived for that purpose fits the Stokes $V$ profiles to two Gaussian functions (one per lobe) and a second order polynomial according to

$$
Y(\lambda, \boldsymbol{a})=a_{0} \mathrm{e}^{-\left(\lambda-a_{1}\right)^{2} /\left(2 a_{2}^{2}\right)}+a_{3} \mathrm{e}^{-\left(\lambda-a_{4}\right)^{2} /\left(2 a_{5}^{2}\right)}+a_{6}+a_{7} \lambda+a_{8} \lambda^{2} .
$$

The parameters $\boldsymbol{a}=\left(a_{0}, \ldots, a_{8}\right)$ were initialised with characteristics of the observational profiles. Constraints were applied to avoid information from pixels, i.e. positions 
on the Sun, with signal below the noise level or which does not fit to the expected Stokes $V$ shape with two lobes. We thus excluded more complicated $V$ profiles, e.g. with three lobes which can arise under complex dynamic conditions. From the fitted analytic $V$ profiles we extract the separation of the lobes and their amplitudes. The lower limit of detectable Stokes $V$ signals in one pixel in the FOV, i.e. the noise level, is $3 \ldots 5 \times 10^{-3} I_{\mathrm{c}}$, where $I_{\mathrm{c}}$ is the average penumbral continuum intensity.

Examples of $B_{\text {eff }}$ as contours over-plotted to the corresponding continuum image are shown in Fig.4.12 The measurable signals are essentially concentrated in the inner and middle penumbra and absent in the outer penumbra except for the region (represented as black contours) in the limb-side penumbra, where a change of polarity has ocurred, i.e. negative values of $B_{\text {eff }}$ are seen.
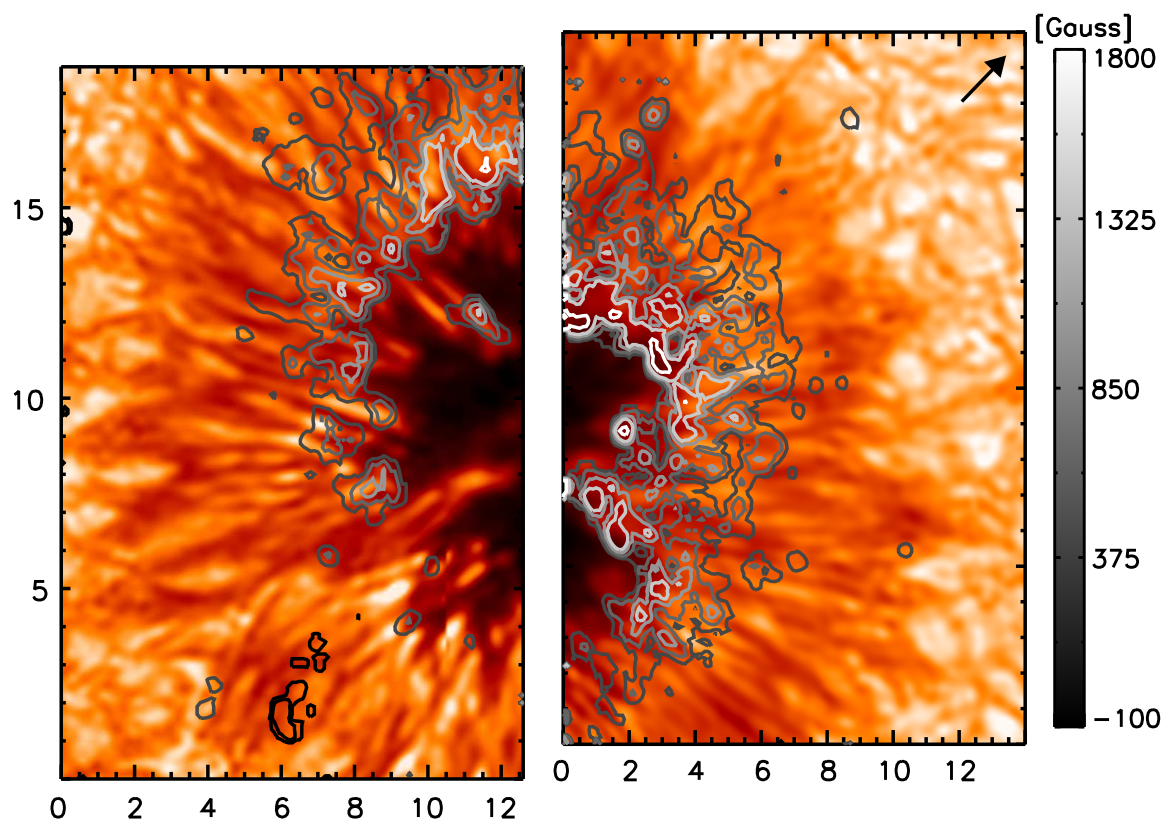

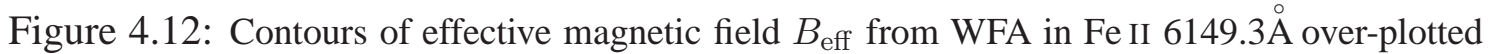
onto continuum image of NOAA 0346 . The arrow points towards disc centre.

\section{From COG method}

In the magnetograms from the COG method (Fig.4.13) the penumbral filamentary structures can be seen, despite the weakness of the Fe II 6149.3 line. At the outer penumbra the magnetic signals are often too low for a reliable measurement. The grainy appearance of the map at scales of $0{ }^{\prime \prime} 2-0^{\prime} .4$ arises from noise due to the weakness of the magnetic signals in this Fe II line.

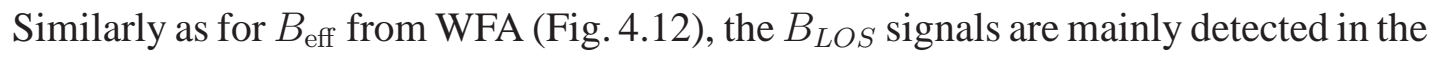
inner and middle penumbra. Also, magnetic fields oriented away from the observer in the limb-side penumbra, indicated by the negative field strengths in the magnetograms, can be interpreted as the horizontal fields of the uncombed magnetic structuring, i.e. changes 


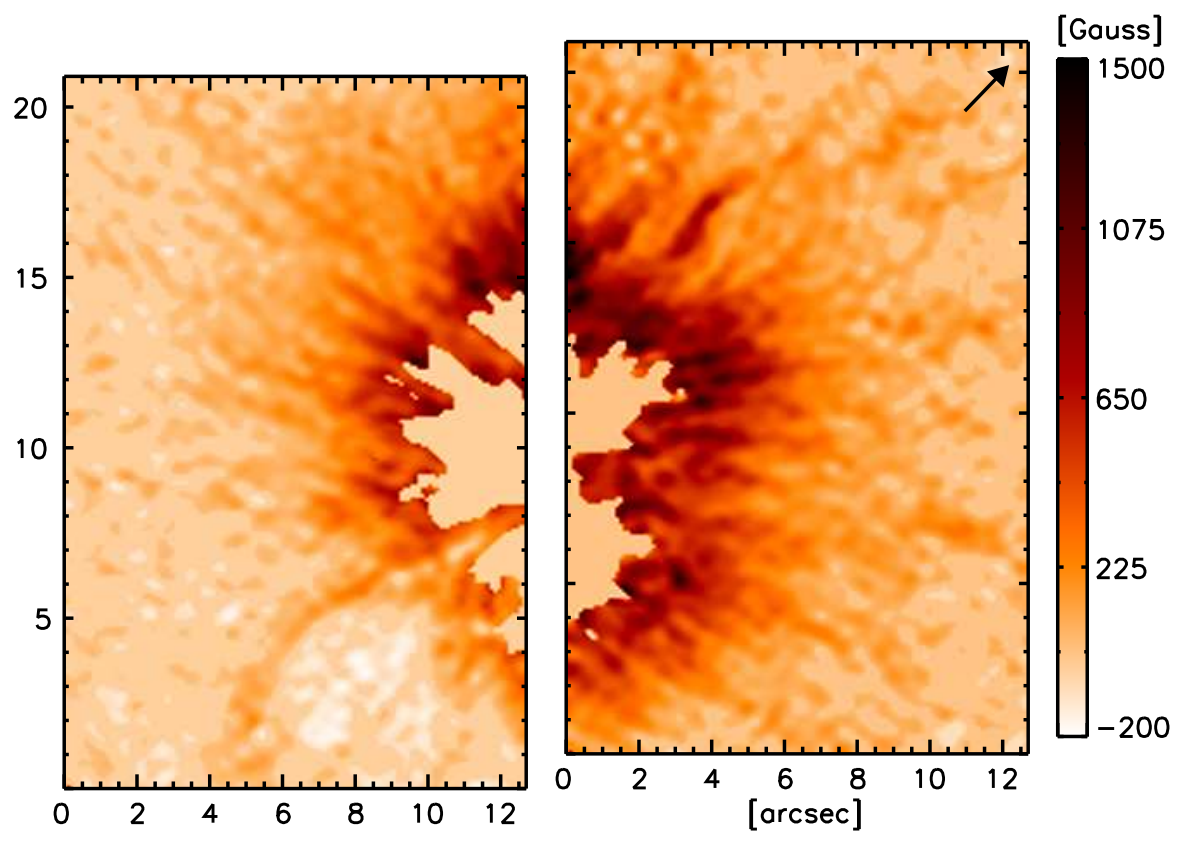

Figure 4.13: LOS magnetic field from COG method in Fe II 6149.3 of NOAA 0346. The arrow points towards disc centre.

of inclination with respect to the vertical (Solanki \& Montavon 1993, see also in Introduction). Close to this negative-polarity region, a long magnetic filamentary structure with $B$ oriented towards the observer and intruding into the umbra can be seen. It is interpreted as a structure with small magnetic field inclination (with respect to the vertical to the solar surface) in the picture of the uncombed fields.

\subsubsection{Magnetic field maps from Fe I 6302.5}

\section{From COG method}

Line-of-sight magnetic field $B_{\mathrm{LOS}}$ maps calculated from the COG method of NOAA 9919 are shown in Fig.4.14. Quantitative values from -500 Gauss up to +2200 Gauss are obtained using Eq.2.53. By close inspection of the $B_{L O S}$ map, we find the following properties:

- $B_{L O S}$ exhibits a filamentary structure, it originates from small scale penumbral features.

- The limb side (left panel) shows an inversion of polarity where one sees filaments in the middle and outer penumbra of negative magnetic field. It is also visible in small-scale negative polarities in the surrounding "quiet" photosphere. They are intersected by positive polarity filaments. The negative fields have LOS components with extremal values of -300 Gauss.

The magnetic characteristics of Fe I $6302.5 \AA$ make this line a powerful diagnostic of $B_{\mathrm{LOS}}$ in penumbrae. 


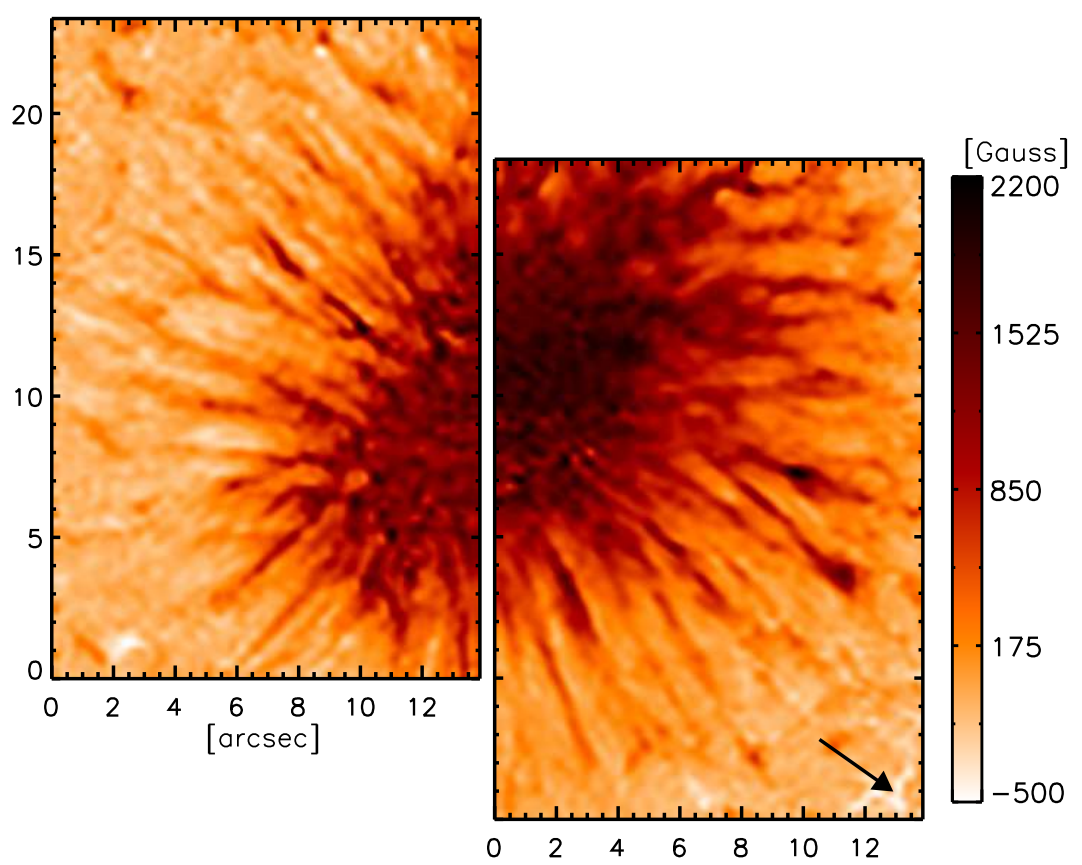

Figure 4.14: Magnetograms of NOAA 9919 obtained applying the COG method in Fe I $6302.5 \AA$. The arrow points towards disc centre.

\section{From the Strong Field Regime approximation}

The SFR approximation provides estimates of the total magnetic field strengths. Fig.4.15 shows an example of a magnetogram obtained in this way. Due to limitations of the algorithm used to fit the Stokes $V$ profiles through expression 4.4 the magnetic map shows areas set to zero where the signal is too low or where no reasonable fits to the measured $V$ profiles could be found.

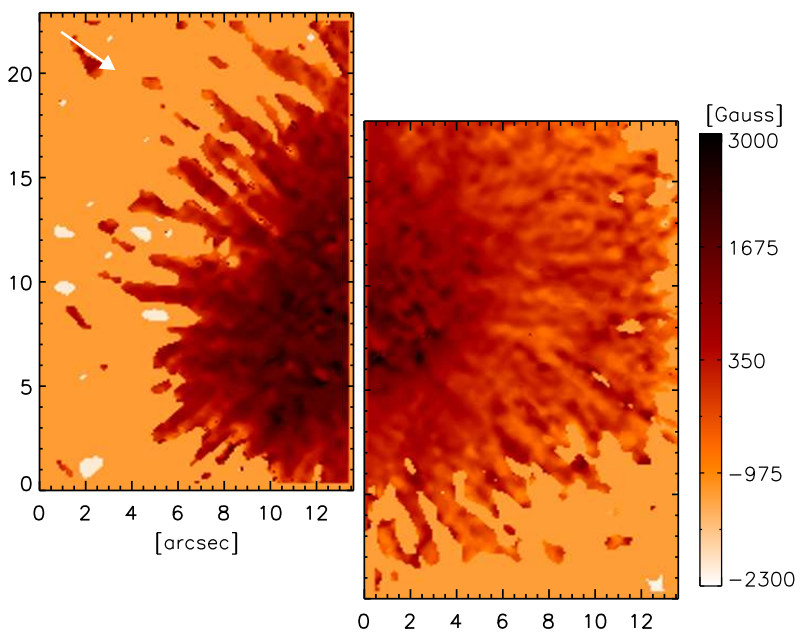

Figure 4.15: Magnetograms of NOAA 9919 obtained from the separation of the $V$ extrema in Fe I $6302.5 \AA$. It is padded with zero shade where the signals are too low for a reliable determination. The arrow points towards disc centre. 
In this magnetogram, the filamentary structure cannot be seen as obviously as found with the COG method above. The values of magnetic field strength run from -2300 Gauss up to +3000 Gauss, the latter field strengths are found in the umbra and very inner penumbra where they are expected. Here again negative polarities found on the limb side of the area are confirmed although they cannot be properly related to any specific penumbral structure.

\subsection{Velocity maps}

Calculations of LOS velocities have been also performed making use of the spectral information contained in the observed profiles of the three spectral lines in use, i.e. the iron pair at $\lambda 6302 \AA$ and the ionised iron line at $\lambda 6149.3 \AA$. Different methods have been applied providing us with various two-dimensional maps. The procedures and the results are described next.

\subsubsection{Bisectors and line minimum}

Bisectors and line minimum positions are used to measure LOS velocities from the Stokes $I$ profiles. At a certain intensity level, the line bisector gives the mean wavelength position of the two intersection points with the line profile and thus measures the Doppler shift. The minima of the measured $I$ profiles were determined with a parabolic fit. Their wavelength positions give also velocities. Figure 4.16 shows the representation of a bisector (in red) for a given intensity profile calculated from three different intensity levels and connected to the line minimum. From its definition, the non-vertical shape of a line bisector entails the existence of asymmetries in the line profile.

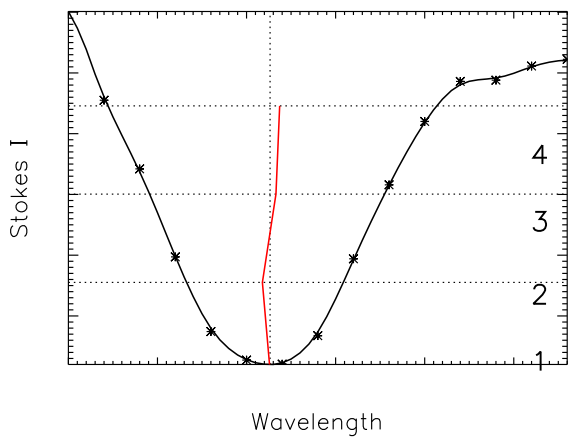

Figure 4.16: Representation of a bisector (in red) of a given Stokes I profile (solid black). The horizontal dotted lines represent the different intensity levels (equivalent to different positions in the atmosphere) where the bisector is calculated. The central vertical (dotted) line marks the wavelength reference position of the line minimum from which wavelength shifts are measured to estimate LOS velocities. The ordinate represents intensity and the abcissa the wavelength direction. 
Intensity profiles from the Fe I $6301.5 \AA$ are used for the calculation of two-dimensional LOS velocity maps. This line is used for two reasons. First, its lower sensitivity to the magnetic field effects (Landé factor $g_{\text {eff }}=1.67$ ) and second, the large range of formation (from 150-400 km, see Fig. 4.9). Thus velocity maps from different intensity levels will trace the velocity field at different heights in the atmosphere. In order to reduce noise, maps from two consecutive observational spectrum scans have been averaged. Results for all four intensity levels are shown in Fig.4.17 and are described below:

- Line-minimum maps (upper-left panels) show an unexpected (and yet unexplained) LOS velocity distribution different to the typical Evershed-flow pattern, i.e. outwards radial flows. At the limb side (left panel) where one would expect strong redshifts, mainly blueshifts are found. Only along the line perpendicular to the centre-to-limb line, strong redshits in the mid-outer penumbra are visible. In the right panel (centre side), blueshifts corresponding to velocities up to $-1 \mathrm{~km} \mathrm{~s}^{-1}$ are located at the outer penumbra 2 The redshifts present in the upper part of the right panel appear to be associated with the intruding dark umbral features seen in the intensity maps.

- LOS velocity maps from intensity level 2 on Fig.4.16, are shown in the upper-right panels. Velocities of the order of $0.5 \mathrm{~km} \mathrm{~s}^{-1}$ are now found in the outer limb-side penumbra. Those, together with the blueshifts in the centre-side penumbra are now recognised as the Evershed radial outflow.

- Continuing with the intensity level 3 on Fig.4.16 (lower-left panel in Fig.4.17), LOS velocity maps show a more extended region of redshifts in the limb-side penumbra with the highest values $\left(\sim 0.8 \mathrm{~km} \mathrm{~s}^{-1}\right)$ at the very outer border and stronger blueshifts corresponding to LOS velocities up to $-1.5 \mathrm{~km} \mathrm{~s}^{-1}$ in the outer, centreside penumbra.

- LOS velocities from the wings of the spectral line (level 4 on Fig.4.16) are shown in the lower-right panel of Fig.4.17. Here, one sees the homogeneous extension of redshifts in the outer limb-side penumbra. The blueshifts in the inner penumbral boundary are also present. In the centre-side penumbra blueshifts have become stronger achieving higher values corresponding to velocities up to $-2 \mathrm{~km} \mathrm{~s}^{-1}$.

From the behaviour shown by the LOS component of the velocity field when tracing its contribution from the different atmospheric layers, one obtains the general picture of a radial Evershed flow which is better seen in deep layers and decreases with height, as reported by Börner \& Kneer (1992, and references therein).

\footnotetext{
${ }^{2}$ The sign of velocity is used in this thesis such that positive velocities correspond to a redshift, i.e. $\Delta \lambda>0$. We thus follow the traditional astronomical use.
} 

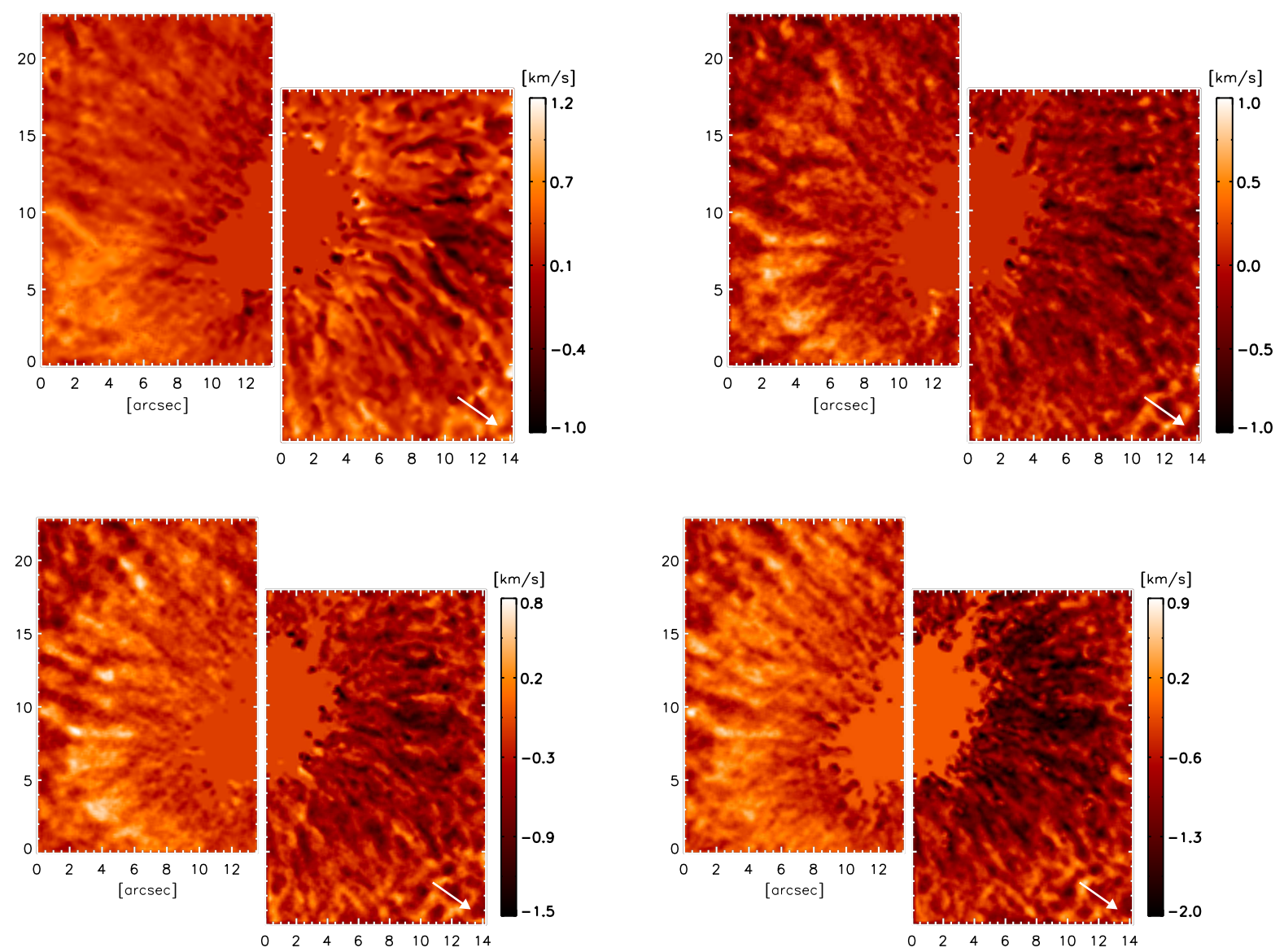

Figure 4.17: LOS velocity maps of NOAA 9919 obtained from bisectors and line minimum position of Fe I $6301.5 \AA$ for four different intensity levels, i.e four different heights in the atmosphere. Values in the umbra are set to zero. The arrows point towards disc centre.

\subsubsection{Stokes $V$ zero crossing}

Another possibility for the estimation of the LOS velocity is through the measurement of the displacement in wavelength of the zero-crossing point of the Stokes $V$ profiles with respect to a given reference wavelength, in this case, that from the mean Stokes $I$ profile from the surrounding photosphere. Results are shown in Fig.4.18.

The LOS velocity maps appear with some noise. Similarly to the magnetic map shown in Fig.4.15, the representation of the distribution of the $V$ zero-crossing shifts is quite sensitive to the strength of the magnetic signal component along the LOS as well as to the fit of the Stokes $V$. The applied fitting procedure is analogous to that explained in Sec.2.4.4, i.e. fitting the lobes to two Gaussian functions and by a second order polynomial.

For a higher reliability the various consecutive scans taken for this sunspot were compared. Thus, the final maps shown in Fig.4.18 are the result of the selection of the common regions of the different scans where the Stokes $V$ profiles could be measured and properly fitted. Therefore, although quantitative values may be affected by noise, the qualitative distribution of the LOS velocities of the magnetic structures is reliable. The zero-crossings appear blueshifted in both the centre-side and the limb-side penumbra and 

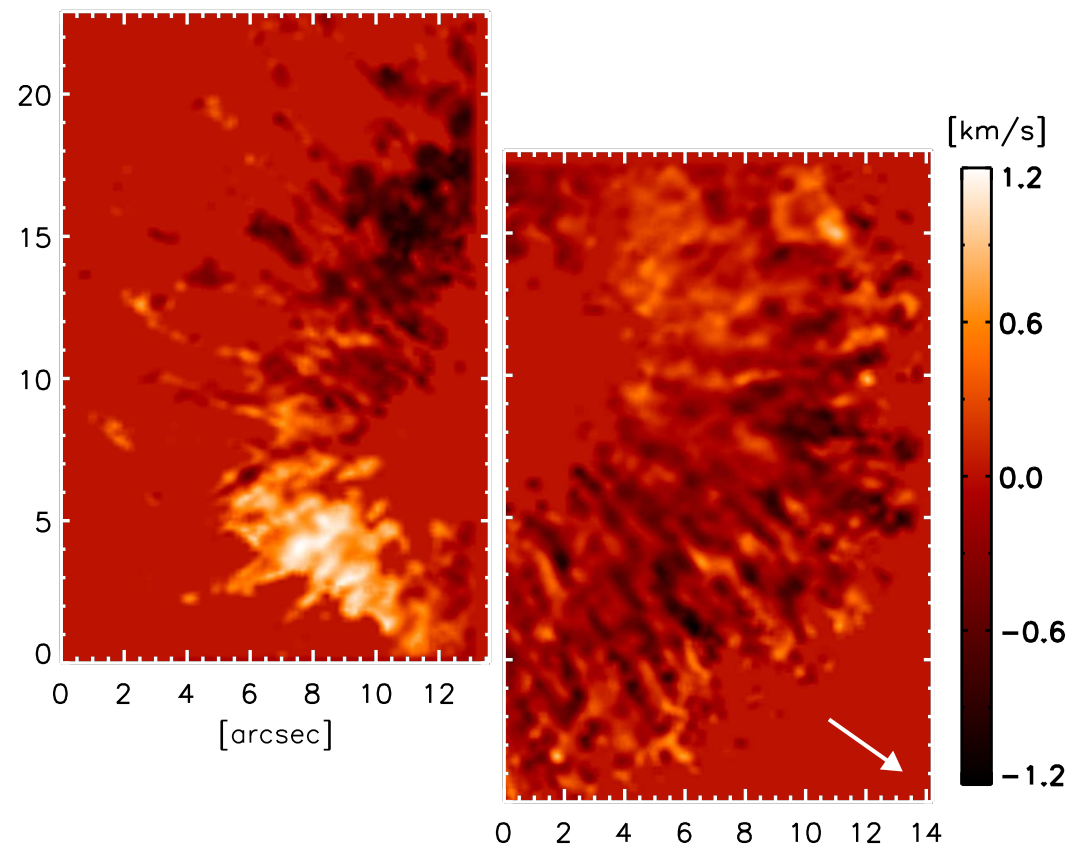

Figure 4.18: LOS velocity map of NOAA 9919 obtained from the Stokes $V$ zero-crossing of Fe I $6302.5 \AA$. Values in the umbra and surrounding photosphere are set to zero. The arrow points towards disc centre.

redshifted in the parts about a line parallel to the limb. This is contrary to the wavelength shifts which are known from the Evershed effect. The antisymmetric velocity maps from the $V$ zero crossing resemble those from the net circular polarisation calculated from infrared lines by Schlichemaier \& Collados (2002) and modelled by Müller et al. (2002). The latter consider a three-dimensional model based on the moving flux tube model (Schlichenmaier et al. 1998). They show that the antisymmetric variation is caused by anomalous dispersion (Faraday effect) and the discontinuity in the azimuthal angle of the magnetic field, which is due to the relative inclination between flux tube and background field. However, this antisymmetry has so far not been observed in visible lines. Therefore, no obvious explanation about this phenomenon can be here given.

\subsubsection{Centre of gravity method}

The velocities along the LOS were also determined with the COG method applied to determine the Doppler shifts from the circularly-polarised observed components, i.e. the average COG position of the $\frac{1}{2}(I+V)$ and $\frac{1}{2}(I-V)$ profiles. The average line position from the ambient non-spot area was taken as reference, i.e. $\bar{v}_{\text {gran }, L O S}=0$.

The results are two-dimensional maps of the line-of-sight components of the velocity field averaged over the atmospheric heights from which they originate. The maps show continuous low noise signal over the whole area of the field-of-view due to the clear $\frac{1}{2}(I+V)$ and $\frac{1}{2}(I-V)$ profiles associated with each pixel, in contrast to the previous maps (Fig.4.18, for example) based on an inhomogenously distributed Stokes $V$ signal. 
Measurements have been performed for all three lines, the Fe I line pair at $6302 \AA$ and the Fe II at $6149.3 \AA$ line. The resulting LOS velocity maps are shown in Fig.4.19 and Fig.4.20.
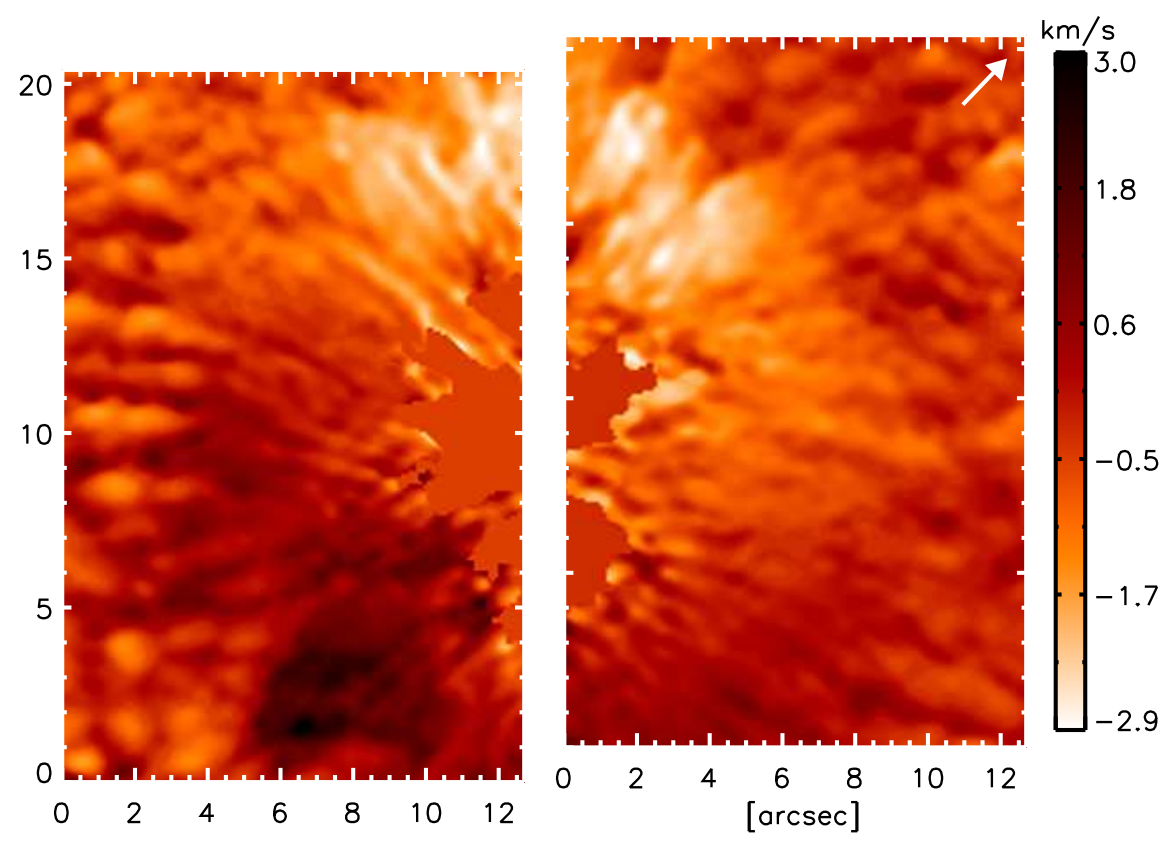

Figure 4.19: LOS velocity map of NOAA 0346 obtained from the COG method for Fe II $6149.3 \AA$. Values in the umbra are set to zero. The arrow points towards disc centre.

LOS velocity maps from the $\lambda 6149.3 \AA$ line on NOAA 0346 (Fig.4.19) show the typical Evershed flow pattern with blueshifts (brighter colors), i.e. flow of material towards the observer, in the centre-side and redshifts (darker colors), i.e. flows away from the observer, in the limb-side penumbra. These wavelength shifts are the result of the radial outflow of penumbral material from the inner to the outer penumbral boundary. Although with smoothed appearence over the whole area, the signal shows the filamentary distribution of the LOS velocity components.

The LOS velocity maps of NOAA 9919 (Fig.4.20) calculated from the neutral iron lines are scaled to the same values from -1.7 up to $1.8 \mathrm{~km} \mathrm{~s}^{-1}$. Some structures (which appear saturated in the representation, mainly in the photosphere) possess higher velocities. These maps show, together with the average Evershed flow pattern, the highly filamentary structure of the LOS velocity field. It is better seen than in the previous maps for NOAA 0346 due to the better seeing conditions during the observations. 

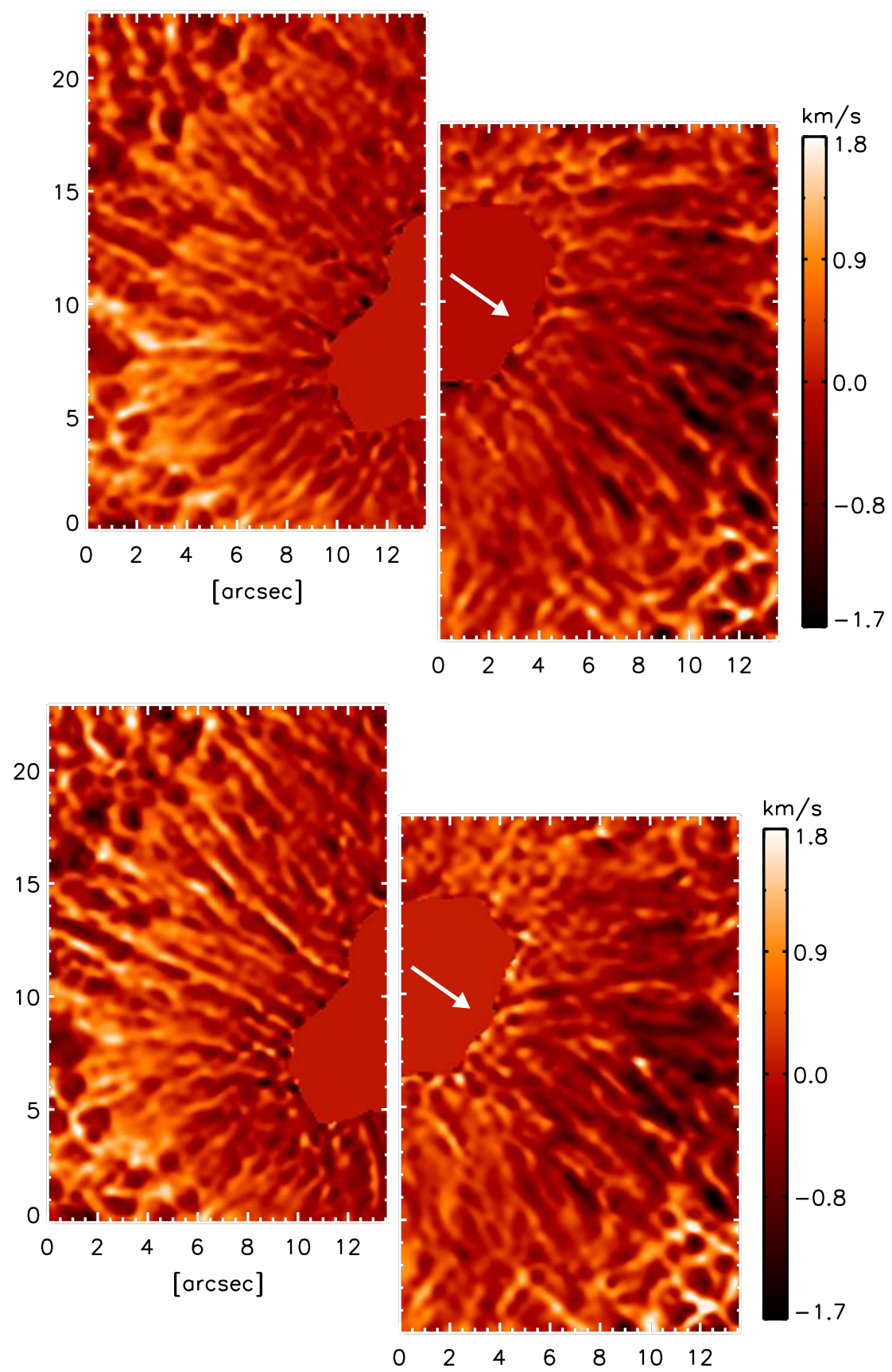

Figure 4.20: LOS velocity map of NOAA 9919 obtained from the COG method for the Fe I lines at $6301.5 \AA$ (upper) and $6302.5 \AA$ (lower). The umbra is padded with zero shade. The arrows point towards disc centre.

\subsection{Comparison of intensity, magnetic field and velocity maps}

Now that the analysis of the intensity maps, and the procedures for the estimation of the LOS magnetic and velocity components through different methods have been described, 
let us combine the information in order to build up a picture of the penumbral atmospheres from the observational point of view. Next, the relations between these physical parameters characteristic of the penumbral medium are treated in detail for the both observed sunspots.

\subsubsection{Fe II 6149.3 observations at $\theta=23^{\circ}$}

Let us now focus on the speckle-reconstructed images (Fig.4.1), the effective magnetic

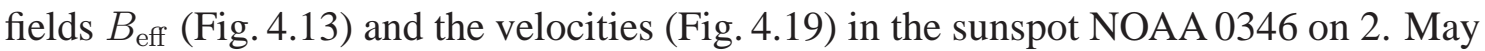
2003, obtained with the COG method in the Fe II 6149.3 $\AA$ line. The WFA for magnetic field determinations gave consistent results (Fig.4.12). Because of its origin from ionised iron, this Fe II line is very sensitive to the temperatures prevailing in the penumbra, where it is weakened compared with the ambient photosphere, and it should not occur in the umbra, where its signal was indeed very low. We find from spectral line modelling that its line centre in the penumbra is formed at heights between $z=-20 \mathrm{~km}$ and $z=+120 \mathrm{~km}$ (above $\tau_{5}=1$ ).
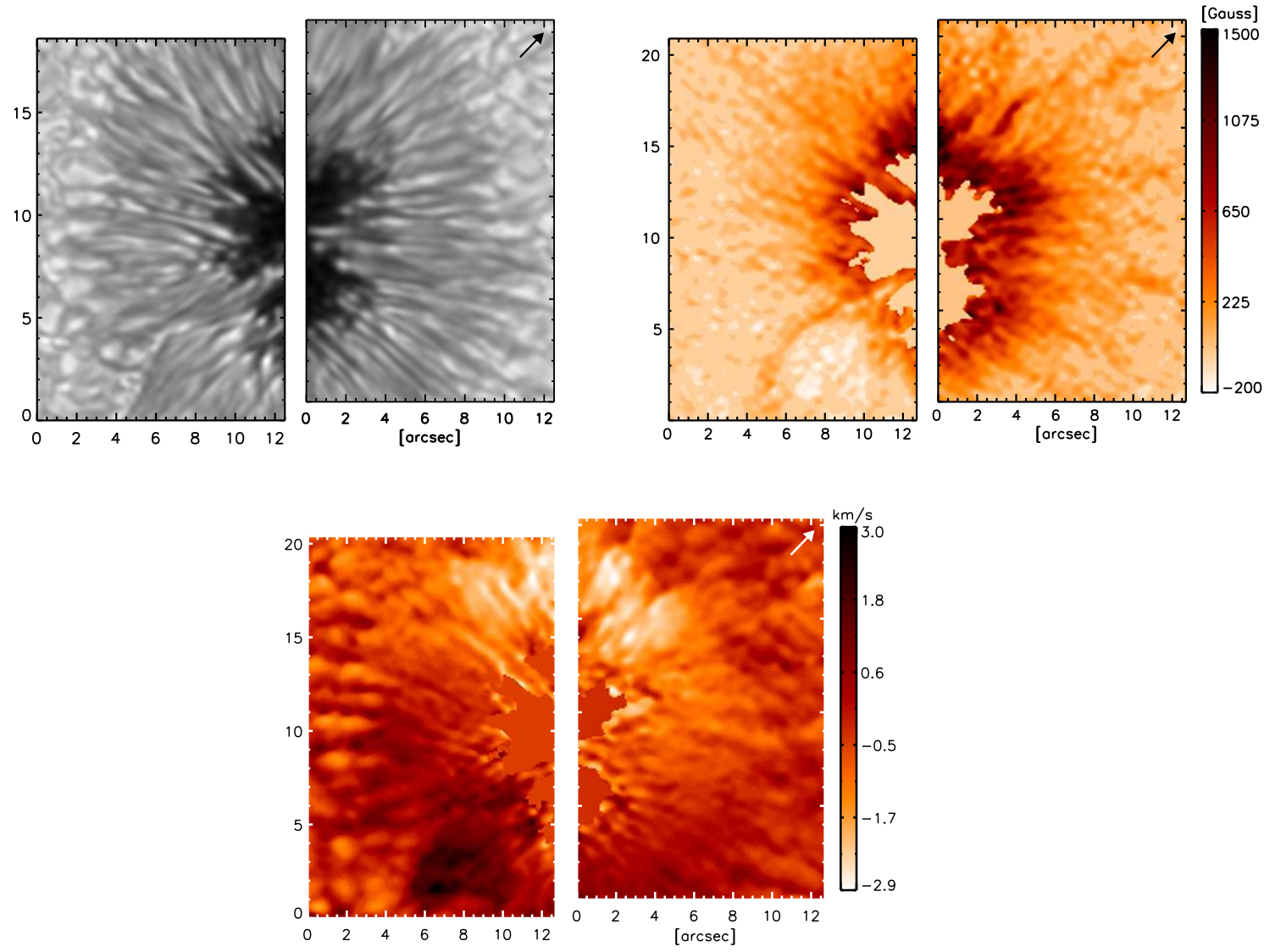

Figure 4.21: Speckle-reconstructed image, LOS magnetic field and LOS velocity maps (from COG method) of NOAA 0346 observed in Fe II 6149.3.

Before discussing details we notice the general properties of the maps: 
1. In both the magnetograms and the velocity maps, the penumbral filamentary structures can be seen, despite the weakness of the Fe II $6149 \AA$ line. At the outer penumbra, the magnetic signals are often too low for a reliable measurement. The dark, dot-like structures near the umbra in the magnetograms of Fig.4.13 are discussed in the list below under item 4. Beyond these, the grainy structure with scales of 0 '.2-0'.4 arises from noise due to the weakness of the magnetic signals in this Fe II line.

2. The magnetic field is oriented towards the observer at the disc-centre side of the penumbra and away from the observer in some parts of the limb-side penumbra.

3. Likewise, the Evershed flow is clearly seen, i.e. as velocities towards (blue shifts) and away from (red shifts) the observer in the centre and limb side penumbra, respectively.

A close inspection of the intensity, velocity and magnetic maps reveals the following special details:

1. The magnetic fields oriented towards the limb (indicated by negative field strengths in the magnetograms of Fig.4.13) at the limb-side penumbra can be interpreted as the more horizontal fields of the uncombed magnetic structuring. At positions where they intrude into the umbra, they are located between bright elongated structures. This behaviour continues outwards: more horizontal fields coincide with dark fibrils or with diffuse structures.

2. Let us now refer to the long magnetic, filamentary structure close to the negative polarity region extended from $(x, y)=\left(5^{\prime \prime}, 2^{\prime \prime}\right)$ in Fig.4.13 until the umbra-penumbra border and intruding into the umbra. From its positive polarity, it is interpreted as a structure with small magnetic field inclination (with respect to the vertical to the solar surface) in the picture of uncombed fields. The lower sharp border of the magnetic feature is located close to strong outflows and follows the border of a dark filament all along its path from the umbra to the outer penumbra. Concerning intensities, the magnetic feature houses bright and dark structures. Thus, we cannot tell conclusively whether fields with small inclination are located in bright or dark structures.

3. In the upper left part of the umbra one notices two bright filaments intruding into the umbra at $(x, y)=\left(11^{\prime \prime}, 13^{\prime \prime}\right)$ in Fig.4.13 The magnetic field component along the LOS is strongest at their ends and between them. This supports again the moving flux tube model with the most vertical magnetic fields at the emerging point bending outwards into a more horizontal direction. Also, the uncombed field picture is confirmed if one interprets the observation of strong LOS field components between the two bright filaments as a signature of more vertical magnetic fields. Generally, near the umbra, the magnetic filaments with strong LOS components are located in dark structures. This however contradicts in some sense item 1 above, where the "horizontal" magnetic field is also located between two bright filaments. 
4. Strong LOS magnetic field components near the border to the umbra are seen as dark dots in the magnetograms of Fig.4.13 They are located at the ends of diffuse bright features, not at the bright penumbral grains in the intensity maps. We may identify them with the umbral ends of emerging flux tubes in the model by Schlichenmaier et al. (1998). In this model, the magnetic field of the tube has the lowest inclination (with respect to the vertical) and the gas inside the tube is hottest at the umbral border.

5. On the disc-centre side, near the border between the penumbra and the quiet photosphere, the picture is less conclusive. The magnetic filaments with strong LOS components may coincide with dark filaments, or with more diffuse or bright structures.

6. The filamentary structuring of the velocity field is best seen near a dividing line across the sunspot perpendicular to the limb-to-centre direction. There, a rapid change of the LOS velocity occurs. On the disc-centre side, blue-shift occurs both in bright structures and in their outward tails. Yet not every bright structure contains flows, and the highest velocities do not occur in the brightest filaments. This behaviour is seen throughout the penumbra, from near the umbra to the border to the normal photosphere. In contrast to the centre side, the positions of the outward flows on the limb side exhibit a systematic difference: There, the LOS velocities are generally largest in dark structures. The interpretation may be that the strongest outflows are concentrated in more "horizontal" magnetic fields. Compared with the ambient, more vertical background field, they occur at positions with strong LOS magnetic components at the limb side and at positions with weak LOS magnetic field components on the centre side. The more vertical background field on the limb side has only a small LOS component. But, on the centre side, this LOS component is larger than the LOS component of the horizontal field. The LOS velocities can attain values of $3 \mathrm{~km} \mathrm{~s}^{-1}$ and thus, accounting for the projection, the velocities may be in the range of $5-7 \mathrm{~km} \mathrm{~s}^{-1}$.

7. The pattern of high LOS velocities consists of more short, only a few arcsec long features with a knotted appearance rather than of elongated fibrils, which is especially well seen in the limb side penumbra.

The inconclusiveness of earlier observational results on the relation between intensity and Evershed flow (item 6 above) thus appears to be an outcome of the viewing angle, i.e. from which side, centre or limb side, the analysed data were taken. The explanation of the systematic difference, that the higher LOS velocities occur at bright features on the centre side and at dark features on the limb side, can again be found with the picture of the moving flux tube in mind: The upflows of hot gas that are seen on the centre side are more vertical. Once the gas has cooled and the magnetic tube has bent into the horizontal direction, the flow will be seen best in dark structures on the limb side. An extension of this model can also explain the shortness of the high-speed LOS flow features (item 7 above). Following Schlichenmaier (2003) it appears reasonable to assume downward bending of the flow channels towards subphotospheric layers within short distances, as had been found by Schlichenmaier \& Schmidt (2000). The magnetic flux tubes carrying the flows resemble then "sea serpents" (Schmidt 2003). 


\subsubsection{Fe I 6302 line pair observations at $\theta=20^{\circ}$}

Let us now turn to the relations between (speckle-reconstructed) broadband intensities, magnetic fields and velocities measured in the Fe I $6302 \AA$ line pair. These observations possess a still better spatial resolution than the ones of FeII $6149.3 \AA$ described above. Also, Fe I 6302.5 is more sensitive to magnetic fields and makes it possible to determine (within the approximations discussed in Sec.2.4.5) the amplitude and polarity of the magnetic fields from the separation of the $V$ extrema as well as the LOS components from COG. It is formed higher in the atmosphere than Fe II 6149.3 and thus reflects different atmospheric properties. Again, for convenient discussion, we combined the relevant images of both halves of NOAA 9919 into Fig.4.22.
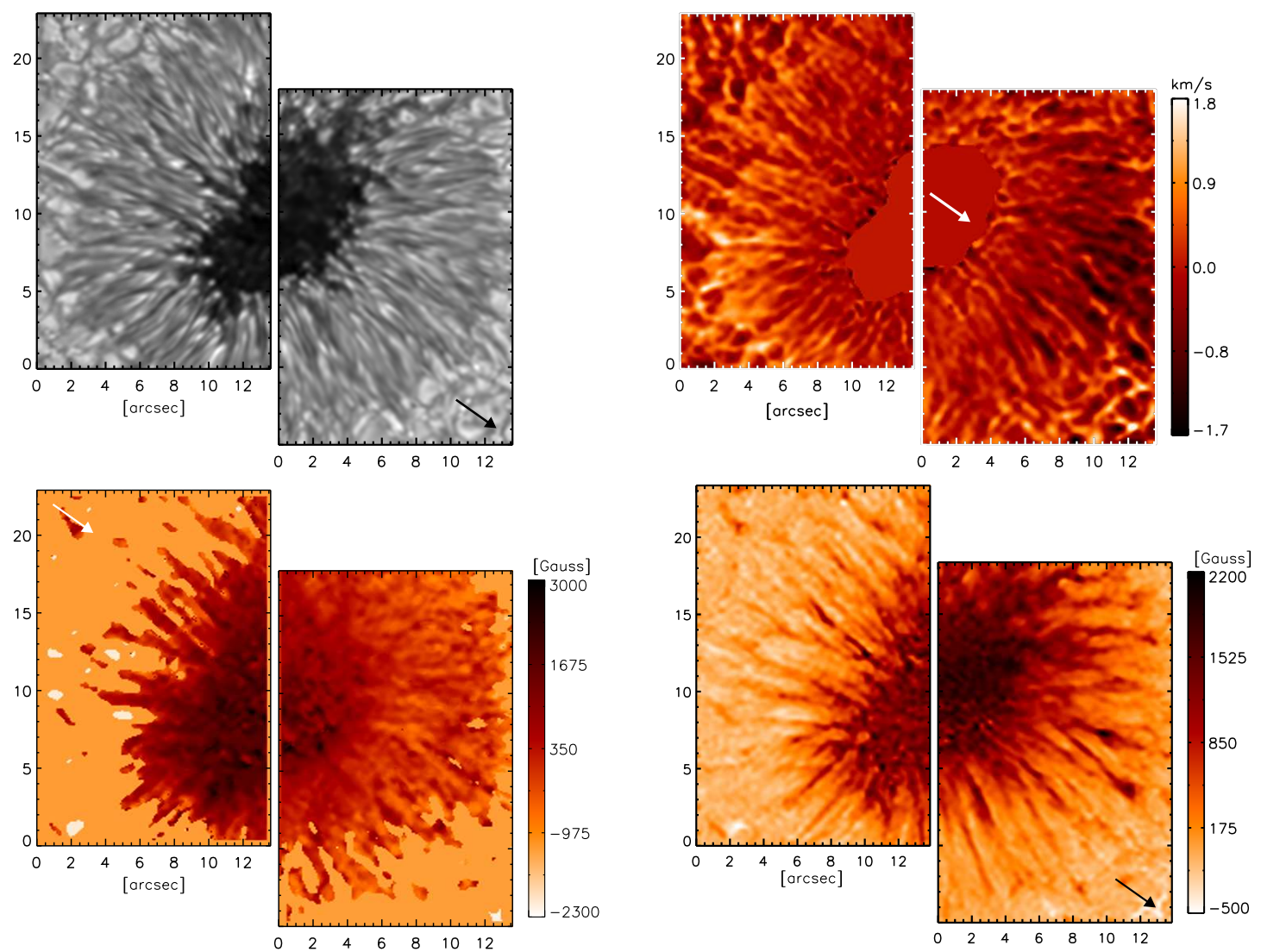

Figure 4.22: Speckle-reconstructed image (upper left), LOS velocity (from COG method in Fe I $6301.5 \AA$, upper right), magnetic field strength from the separation of $V$ extrema (lower left) and LOS magnetic field (from COG method, lower right) of NOAA 9919 observed in the Fe I 6302 line pair. 
By close inspection, the following properties are found:

1. The LOS magnetic field calculated from COG (lower right panels of Fig.4.20) shows an inversion of polarity at the limb side where one sees negative magnetic field filaments in the middle and outer penumbra (apart from small-scale negative polarity fields in the ambient "quiet" photosphere). They are intersected by positive polarity filaments. The negative fields have LOS components with extremal values of -300 Gauss, and coincide with dark intensity structures in the continuum (or broadband) images. The positions of negative polarity fields, i.e. of their LOS components, at the limb side in dark intensity structures coincide with the outward velocity described in item 5 below.

2. Again on the limb side, strong LOS magnetic fields of 1500-2500 Gauss are found in dark intensity filaments, yet not in their darkest parts but rather displaced to the borders to bright structures. We recall the similar results from the $\lambda 6149.3$ line observations (item 3 in Sect.4.4.1). In any case, on the limb side, strong LOS magnetic field components are not found in bright structures. These certainly contain also magnetic fields, but with weaker LOS components.

3. In the centre-side penumbra, the strong LOS magnetic field components are located in dark intensity structures, or in diffuse intensity patterns.

4. Negative polarities are also found from the $V$ amplitude separations, but not in such fine detail as from the COG method. When comparing the LOS components with the field strengths determined from the separation of the $V$ extrema one notices that close to the line perpendicular to the limb-to-centre direction and crossing the centre of the umbra the fluctuations of the field amplitude and of the LOS component coincide spatially. At locations towards the limb, this coincidence is mostly preserved, but not always. However, surprisingly, at locations towards the disc centre there exists an anti-correlation between fluctuations of the field amplitude and of the LOS component. A possible explanation lies in (anti-)correlated fluctuations of the field strength and the field inclination (with respect to the vertical) which have no influence at positions of the line perpendicular to the limb-to-centre direction and are less perceptible on the limb side of the penumbra. The anti-correlated fluctuations of field strength and field inclination were already found, from lower spatial resolution data, by Lites et al. (1993) and Westendorp Plaza et al. (2001a) and were discussed by Martínez Pillet (1997).

5. Consistently with the finding for the Fe II 6149.3 observations, the outward Evershed flow on the disc-centre side occurs at positions of bright filaments in the continuum images or at their somewhat weakened tails, while on the limb side the outward flow is definitely located in dark structures. The reverse is not true: Not all bright structures (on the centre side) exhibit strong outflows.

6. The outward velocities, i.e. their LOS components, are smaller than those found in Fe II 6149.3, they rarely attain values larger than $1.5 \mathrm{~km} \mathrm{~s}^{-1}$. Only a small part of this difference can be ascribed to observation at a smaller heliocentric angle, i.e. at $20^{\circ}$ compared to $23^{\circ}$ for the $\lambda 6149.3$ observations, or because the data are from 
different sunspots. The difference reflects the decrease of the Evershed flow with height which is observed in the bisector velocity maps (Fig.4.17) and also reported by Börner \& Kneer (1992, and references therein).

7. The relation between velocities and intensities in the line core of $\lambda 6301.5$ (Fig. 4.10 lower panels) is less clear. On the centre side, large velocities can occur where the line core intensity is less bright than the neighbourhood. This likely reflects the fact that the information about the velocities, measured from COG, stems from deeper layers than the 6301.5 line core intensities.

8. The positions of the Evershed flow and the strong LOS magnetic field components, close to each other, but not fully coinciding, in dark intensity filaments at the limb side, discussed in items 2 and 5 above, are not inconsistent. The flow channels are likely smaller than the spatial resolution of the observations and strong, more vertical magnetic background fields need not necessarily coincide with them. On the limb side, the velocities are perceptible only when the flow channels are bent into a more horizontal direction ore even downward, when the gas, originally hot and rising, has cooled down.

In general, the high-spatial-resolution data analysed here confirm the uncombed magnetic field structure of penumbrae. Title et al. (1993) and Bellot Rubio et al. (2003), among others, found that the more horizontal flux tubes carry the Evershed flow. The latter authors applied inversion techniques to full Stokes profiles of the magnetically sensitive infrared Fe I lines at $1.565 \mu \mathrm{m}$. Their data had lower spatial resolution than the ones presented here and the authors concluded indirectly concerning the magnetic field and velocity structure by adopting two-component models for the inversions. Our highresolution data are compatible with this picture.

\subsection{Intensity, LOS velocity and LOS magnetic field cor- relations}

One open and strongly debated question concerning penumbrae is the correlation between the kinematics and magnetism with the visible penumbral structures, i.e. bright and dark filaments. Different works have been carried out trying to find out possible relations. About the correlations between intensities and the Evershed flow, Wiehr \& Degenhardt (1992), Lites et al. (1993), Rimmele (1995), Stanchfield et al. (1997) and Tritschler et al. (2004) conclude that the Evershed flow is mainly located along the dark structures. However, Wiehr \& Stellmacher (1989), Lites et al. (1990), Schlichenmaier and Schmidt (2000), Hirzberger \& Kneer (2001) find only small correlation or no correlation at all. In most of the cases observations achieved rarely resolutions better than $1^{\prime \prime}$.

Taking advantage of the high spatial resolution characteristic of the velocity, magnetic and intensity maps described above it makes sense to perform again a study of possible correlations between the different parameters associated to the penumbral structure at small scales. The procedure consists in the comparison of profiles obtained from azimuthal cuts on the various maps and the estimation of a correlation coefficient which 
expresses the degree of agreement between the curves. For two sequences of time, or space, $X_{i}, Y_{i}, i=1, \ldots, N$, the correlation coefficient is defined by

$$
C_{X Y}=\left(\sum_{i=1}^{N}\left(X_{i}-\bar{X}\right)\left(Y_{i}-\bar{Y}\right)\right)\left(\sum_{i=1}^{N}\left(X_{i}-\bar{X}\right)^{2} \sum_{i=1}^{N}\left(Y_{i}-\bar{Y}\right)^{2}\right)^{-\frac{1}{2}}
$$

\subsubsection{Intensity vs. velocity field}

Figures 4.23 and 4.24 show examples of intensity and LOS velocity ( $v_{\text {LOS }}$ ) profiles along the azimuthal direction for three different azimuthal cuts, i.e. inner, mid and outer penumbra (indicated by the black curves on the reconstructed broad-band images on the left side). The $v_{\text {LOS }}$ profiles (curves in blue) are extracted from the velocity maps calculated with the COG method (Fig.4.20, upper panel) and the intensity profiles (curves in black) from the narrow-band images at continuum level (Fig.4.10, upper panel). The latter are represented as $\Delta \mathrm{I} / \mathrm{I}_{\text {mean }}$, i.e. fluctuations of intensity around the mean intensity value along the azimuthal cut. Thus, structures with intensity value above zero will be referred as bright structures and below zero as dark structures. The correlation coefficient is esti-
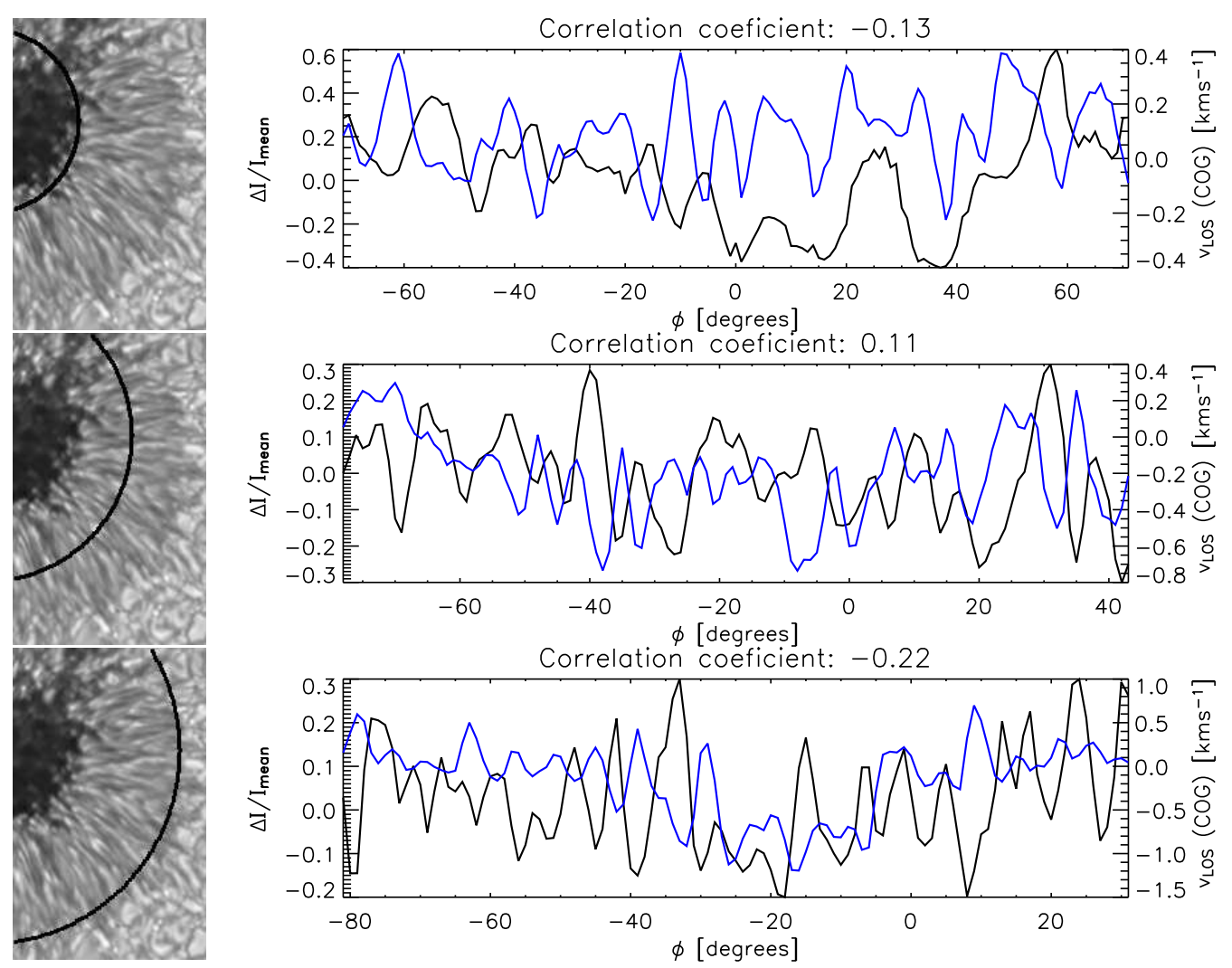

Figure 4.23: Representation of azimuthal cuts of intensity at continuum level (in black) and LOS velocities (in blue), from COG method measurements in Fe I $6301.5 \AA$ for three different radial positions in the centre side: inner (upper panel), mid (middle panel) and outer (lower panel), as denoted by the black curves on the broad-band reconstructed images on the left side. The data are taken from NOAA 9919. 
mated from the comparison of the intensity curves with LOS velocities in absolute value, $\left|v_{\mathrm{LOS}}\right|$. Thus, the factor will refer to the degree of correlation between bright/dark structures with the strength of the LOS velocities, independently of flows towards or away from the observer (note that curves are represented with signed values, positive values indicating flows away from the observer). Then, positive correlation coefficients tell about relations between $\left|v_{\mathrm{LOS}}\right|$ with bright structures, and negative coefficients about relations between $\left|v_{\mathrm{LOS}}\right|$ with dark structures.
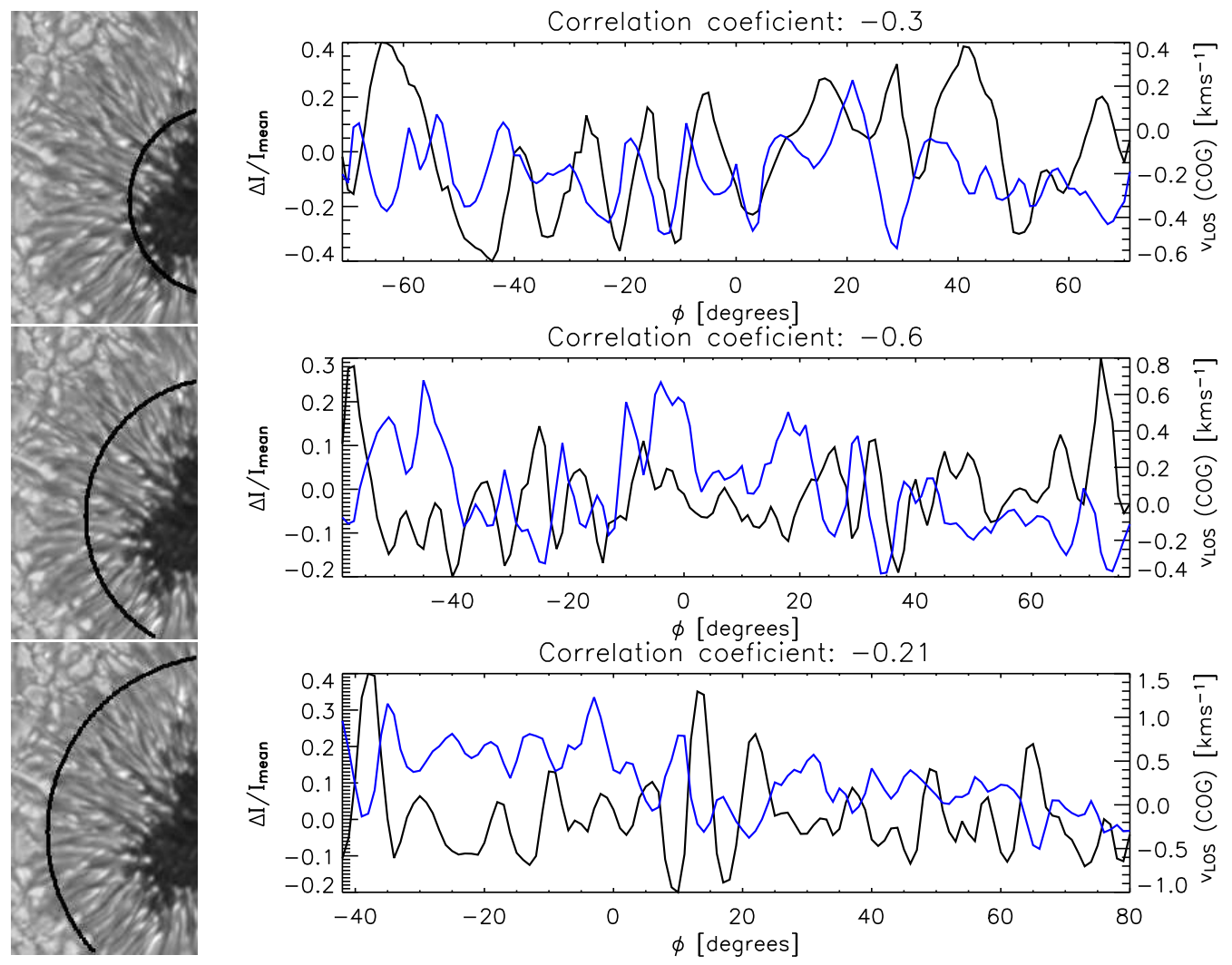

Figure 4.24: The same as for Fig.4.23 but for the limb-side penumbra.

For the centre-side penumbra (Fig.4.23) the correlation coefficient shows variable, but low, values from the inner to the outer penumbra. However, when one refers to the curves there represented, what one indeed sees is that the highest LOS velocities towards the observer (thus negative) are associated with bright structures, better seen in the mid and lower panels (for middle and outer penumbra). Thus, the correlation coefficient seems to give a poor average value which cannot account for such a variety of distributions in intensities and velocities. For the limb-side penumbra (Fig.4.24) one finds the opposite behaviour. The highest LOS velocity (positive) values appear associated with dark structures, which was already pointed out in Sec.4.4.2.

\subsubsection{Intensity vs. magnetic field}

Figures 4.25 and 4.26 show examples of intensity and LOS magnetic field profiles along the azimuthal direction for three different azimuthal cuts. The LOS magnetic field $\left(\mathrm{B}_{\mathrm{LOS}}\right)$ 
profiles are extracted from magnetograms calculated from the COG method (Fig.4.14) and, again, the intensity profiles from the narrow-band images at continuum level (Fig.4.10, upper panel).
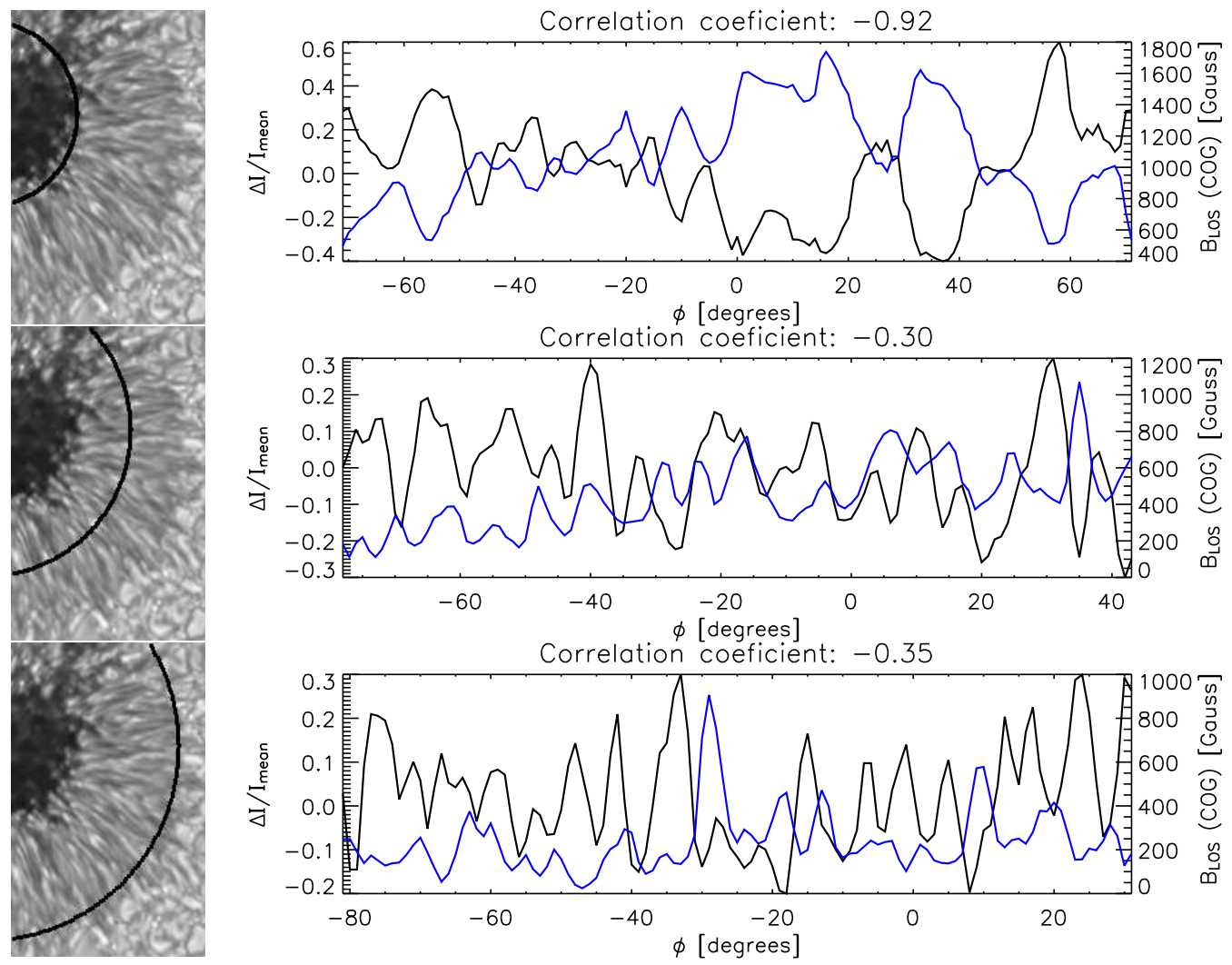

Figure 4.25: Representation of azimuthal cuts of intensity at continuum level (in black) and LOS magnetic field (in blue), from COG method measurements in Fe I 6302.5, for three different radial positions in the centre side: inner (upper panel), mid (middle panel) and outer (lower panel), as denoted by the black curves on the broad-band reconstructed images on the left side.

The strong anti-correlation shown by the intensity and $\mathrm{B}_{\mathrm{LOS}}$ in the very inner penumbra in both, the centre and the limb side, is expected due to the proximity to the umbral border. In this case, the negative correlation coefficient accounts for the anti-correlation of intensity and field strength, and it is a general behaviour all along the azimuthal cut. For mid penumbra, also on both sides, the degree of correlation also takes negative values (correlation of $\mathrm{B}_{\mathrm{LOS}}$ with dark structures). But it is only when one compares in detail the curves that one finds the interesting fact that the strongest $\mathrm{B}_{\mathrm{LOS}}$ do not coincide with the darkest structures but somehow slightly displaced, as it was already pointed out in Sec.4.4.2. At the outer centre-side penumbra the strongest magnetic fields appear to coincide with structures of intermediate brightness (close to zero in the $\Delta \mathrm{I} / \mathrm{I}_{\text {mean }}$ representation). In the outer limb-side penumbra a great variety of brighter structures with some ( 200-400 Gauss) $\mathrm{B}_{\mathrm{LOS}}$ signal, dark structures with $\mathrm{B}_{\mathrm{LOS}} \sim 300$ Gauss and dark structures with $\mathrm{B}_{\mathrm{LOS}}$ of negative polarity, i.e. magnetic fields with large inclination, can be found. 

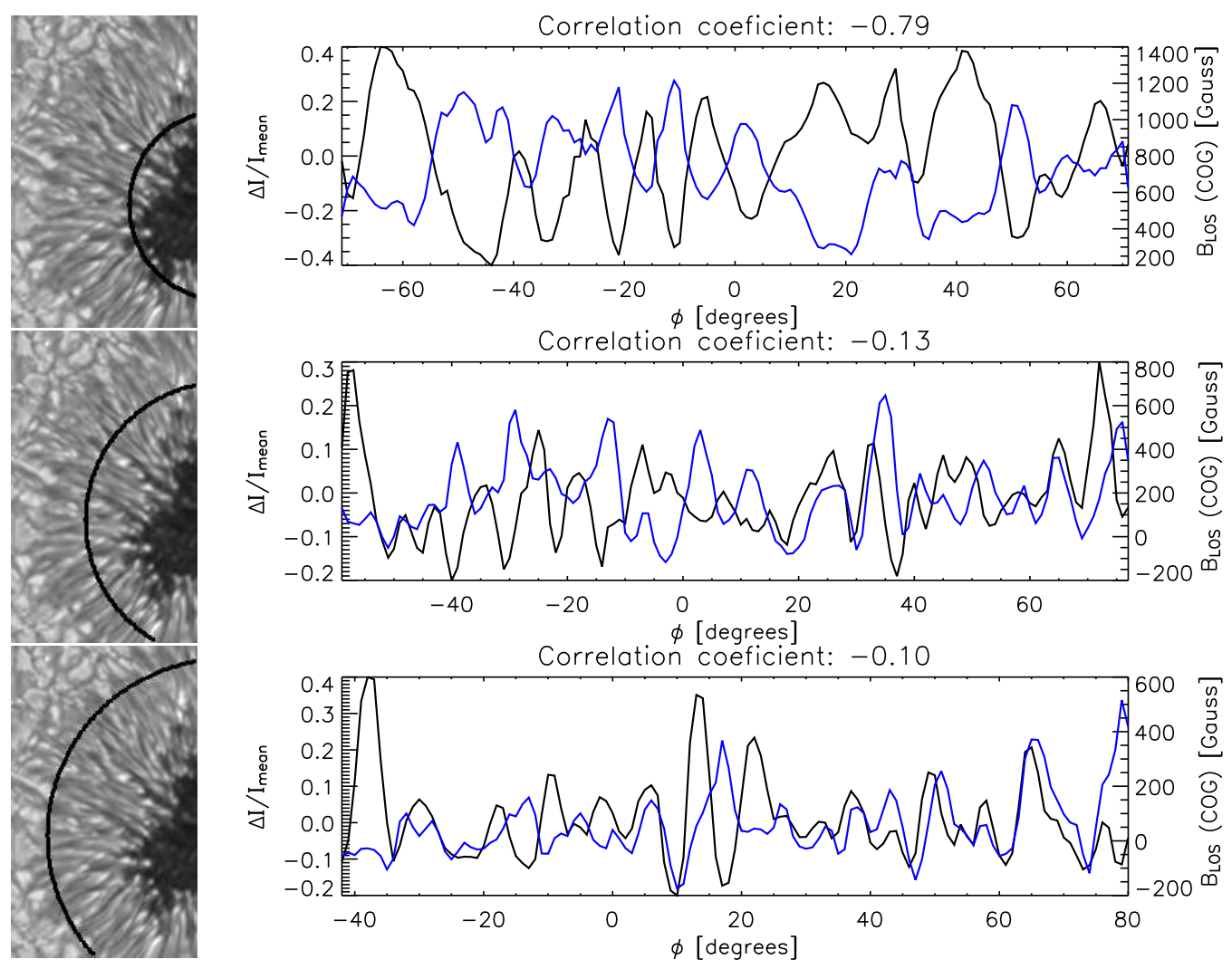

Figure 4.26: The same as for Fig.4.25 but for the limb-side penumbra.

\subsubsection{Magnetic field vs. velocity field}

Finally, the comparison between $\mathrm{B}_{\mathrm{LOS}}$ and $v_{\mathrm{LOS}}$ can be performed in the same manner, i.e. comparing profiles obtained from various azimuthal cuts on magnetograms and velocity maps. Examples for inner, mid and outer penumbra are shown in Fig.4.27 for the centre side and Fig.4.28 for the limb side. Here it is demonstrated explicitly once more how the estimation of a correlation coefficient (represented for all azimuthal cuts in Fig. 4.29) cannot account for the variety of associations that can be found within the penumbra. Only after a detailed study one can see that in the centre side, the strongest (negative) $v_{\mathrm{LOS}}$ values (towards the observer) correspond to low $\mathrm{B}_{\mathrm{LOS}}$ signal, i.e small LOS component of the magnetic fields, i.e. magnetic fields with low strength or very inclined. On the limb side, the strongest positive $v_{\text {LOS }}$ values (away from the observer) coincide with the $\mathrm{B}_{\mathrm{LOS}}$ signal of inverted polarity, i.e. very much horizontal fields. These can be interpreted then as evidences of the spatial coincidence of the Evershed flow with the strongly inclined magnetic field visible in the mid and outer penumbra, which was already proposed by Kinman (1952), Maltby (1964), Schröter (1965), Title (1993), Rimmele (1995), Schlichenmaier \& Schmidt (2000) and recently demonstrated by Bellot Rubio et al. (2003) from data taken in the infrared. Also the displacements should be emphasized here which often occur between the $\mathrm{B}_{\mathrm{LOS}}$ signal and the $v_{\mathrm{LOS}}$ signal. 

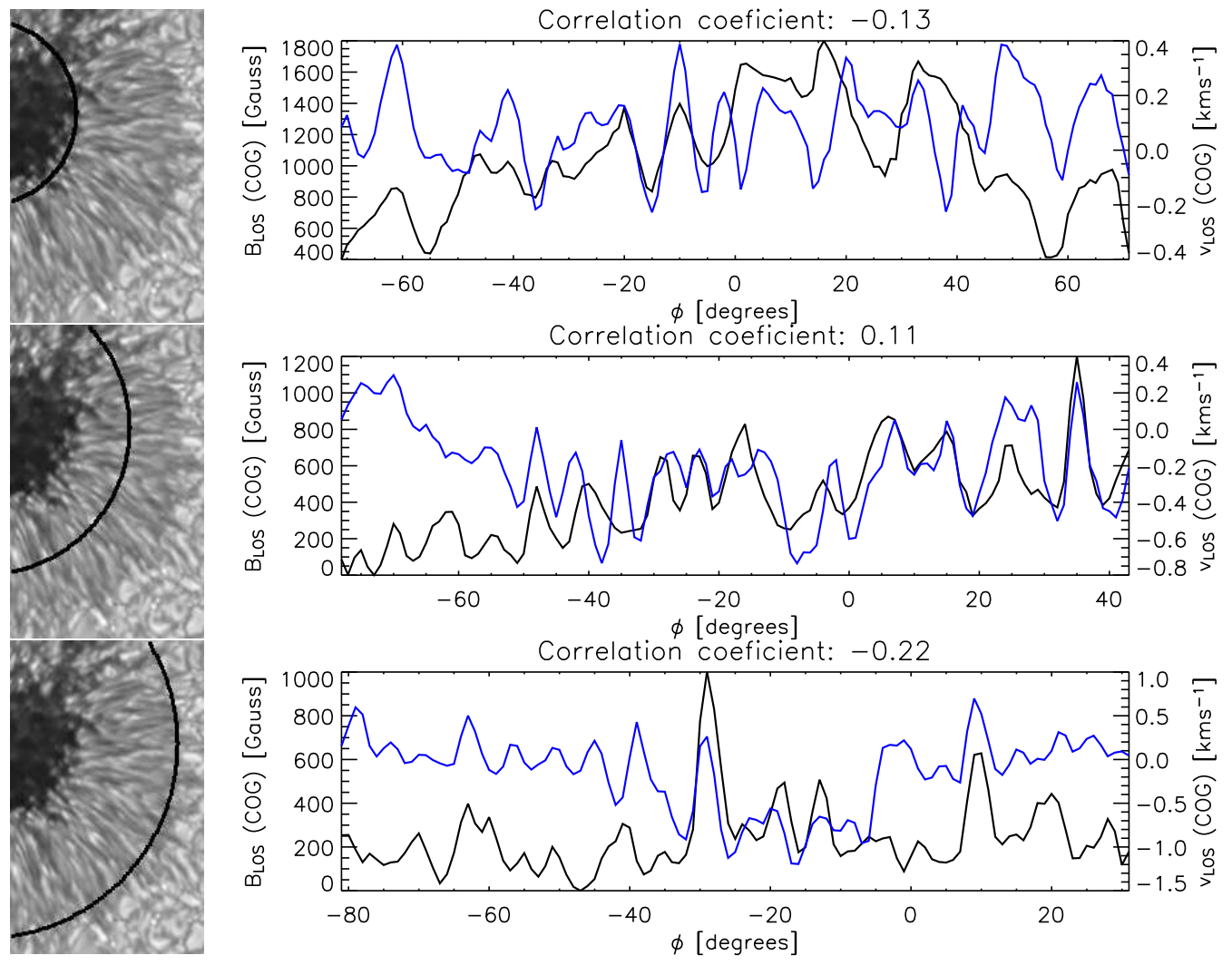

Figure 4.27: Representation of azimuthal cuts of LOS magnetic field (in black, from $\lambda$ 6302.5) and LOS velocities (in blue, from $\lambda$ 6301.5), both from COG method measurements, for three different radial positions in the centre side: inner (upper panel), mid (middle panel) and outer (lower panel), as denoted by the black curves on the broad-band reconstructed images on the left side. 

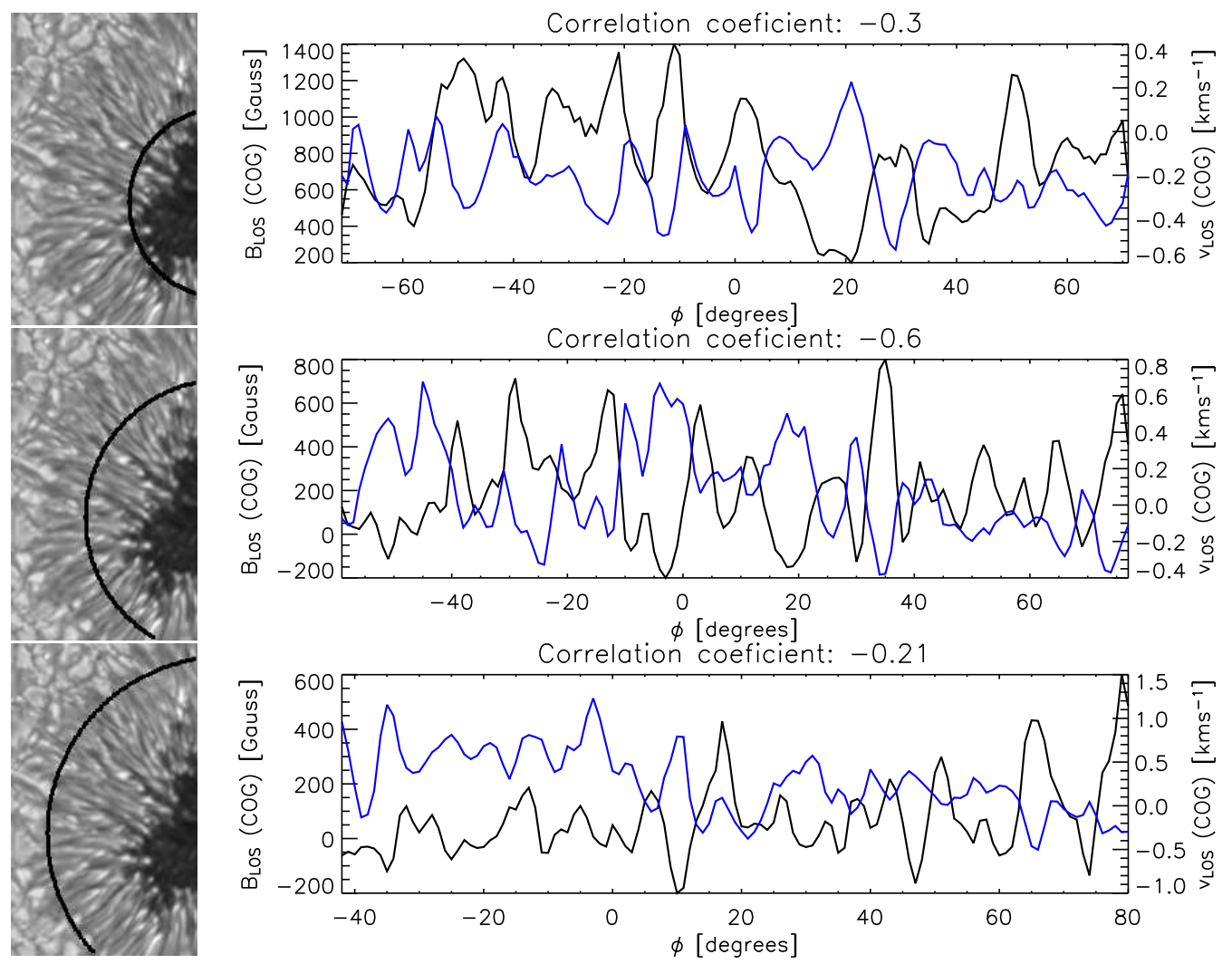

Figure 4.28: The same as for Fig.4.27but for the limb-side penumbra.
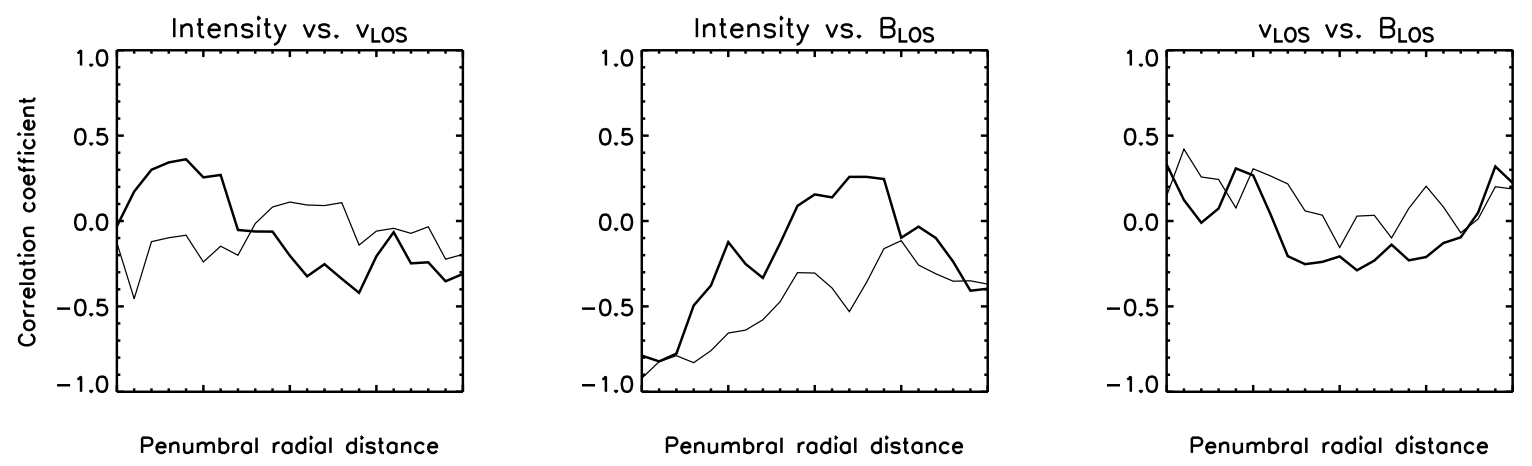

Figure 4.29: Variation of the estimated correlaction factor with radial distance within the penumbra from the comparison of intensity vs. LOS velocity, intensity vs. LOS magnetic field and LOS velocity vs. LOS magnetic field, respectively. Thick curves denote estimations for the centre-side penumbra and thin curves for the limb side. For more detailed information see the text. 



\section{Numerical modelling}

As already pointed out above, the shape of the observed Stokes profiles carries much information about the magnetism and dynamics of the structures where they are formed. To extract this information a synthesis of profiles simulating the observed ones is required. In this chapter, a numerical modelling of the penumbral structures by means of radiative transfer calculations in penumbral atmospheres is presented. To synthesise Stokes profiles a numerical code developed at the Universitäts-Sternwarte Göttingen is used. The one-dimensional radiative transfer problem along a given ray is solved assuming local thermodynamic equilibrium (LTE) (see also Bello González et al. 2005). As will be seen, any attempt to reproduce the asymmetries shown by the observed penumbral Stokes profiles requires strong changes in velocity and in the magnetic field inclination across the atmosphere, i.e. it requires a scenario where at least two different atmospheres coexist within the observational resolution element.

\subsection{Motivation}

\subsubsection{Asymmetries in the observed Stokes profiles}

Intensities, magnetic fields and velocity fields of the penumbral fine-structure have so far been analysed in Chapter 4 from the two-dimensional maps of high spatial resolution. The latter were obtained from the application of different methods of analysis to the observed sunspot area, using the spectropolarimetric information contained in the data. In the following such valuable information is used more explicitly through the study of the individual Stokes $I$ and $V$ profiles in penumbrae.

Stokes profiles in penumbrae are mainly characterised by their asymmetries, actually caused, as will be seen next, by gradients and variations of the magnetic and velocity fields in the penumbral atmosphere. These asymmetries have been recognised since long by many researchers (e.g. Beckers \& Schröter, 1969) and much work has been devoted to this topic, mainly based on spectrographic observations of data with high spectral resolution, but only moderate spatial resolution. Higher spatial resolution data provide Stokes profiles formed in structures that, although they may not be fully resolved, exhibit more detailed information on physical processes in small-scale structures. Some examples of observed penumbral Stokes profiles in the Fe I line pair are given in Figs.5.15.2 and 5.3 in the Fe II line in Figs. 5.4 and 5.5 . 

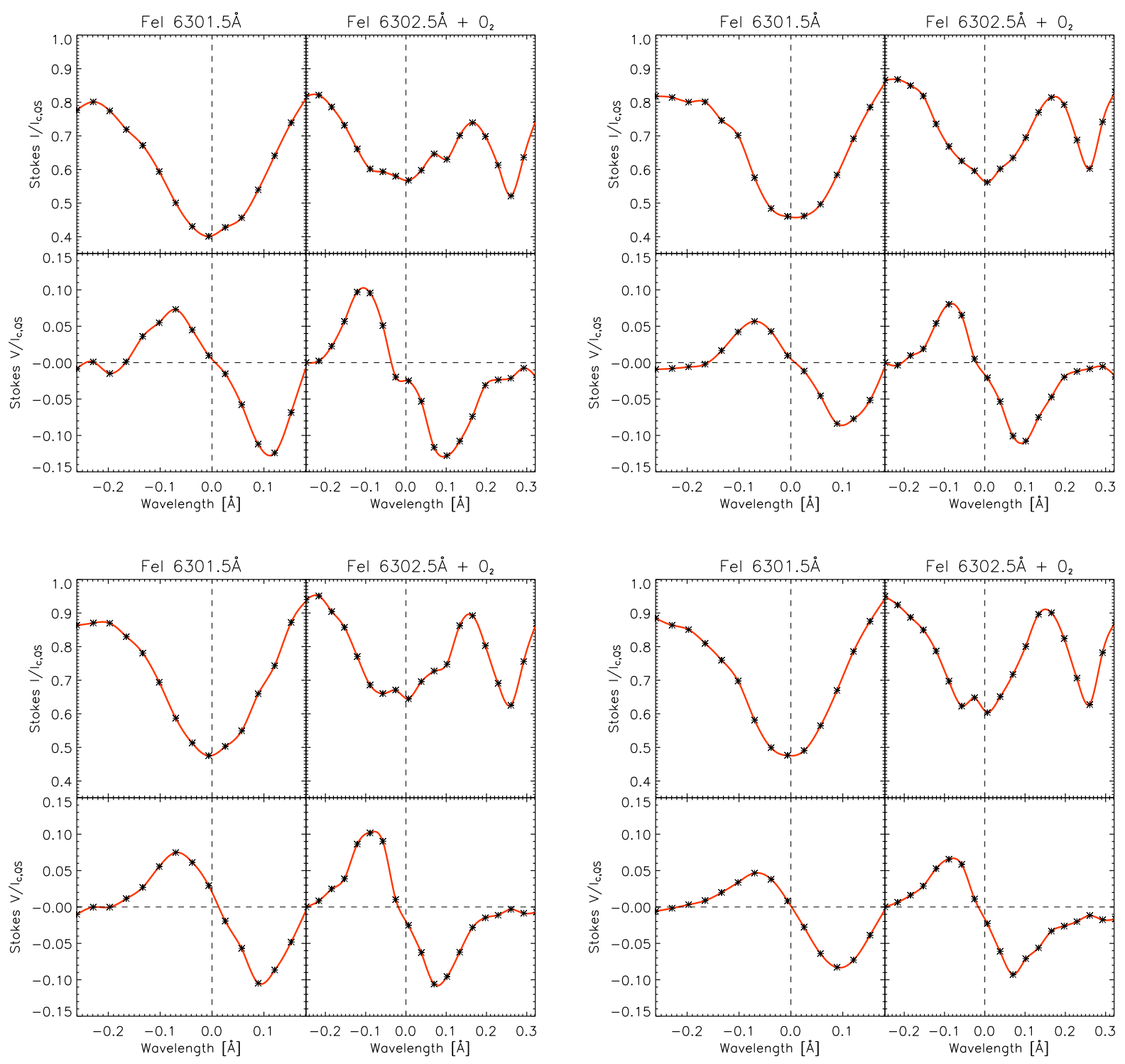

Figure 5.1: Examples of asymmetries in Stokes $I$ and $V$ profiles of the $\mathrm{Fe} I$ lines at $6302 \AA$ in the centre-side penumbra. Upper left panels represent profiles for a bright penumbral grain in the umbra-penumbra border, coordinates $(x, y) \sim\left(1^{\prime \prime} .5,10^{\prime \prime} .5\right)$ in the broad-band reconstructed images (Fig.4.1 lower panel, right). Upper right panels correspond to a bright (locally) penumbral filament in the mid penumbra, coordinates $(x, y) \sim\left(3^{\prime \prime} .5,8^{\prime \prime}\right)$. Lower left panels show profiles for a bright penumbral grain in the inner penumbra, coordinates $(x, y) \sim\left(4{ }^{\prime \prime} .5,11^{\prime \prime} .5\right)$. Lower right panels show profiles of another (locally) bright filament in the mid penumbra, coordinates $(x, y) \sim\left(5^{\prime \prime} .5,10^{\prime \prime}\right)$. Red curves are interpolation to the observed scanning-positions (stars) along the spectral lines. Vertical lines represent the wavelength reference, i.e. line centre of the averaged Stokes $I$ profiles in the surrounding photosphere. The narrow spectral feature on the red side of $\lambda 6302.5 \AA$ (at $\Delta \lambda \approx 0.25 \AA$ from the line centre) is a telluric $\mathrm{O}_{2}$ line. 

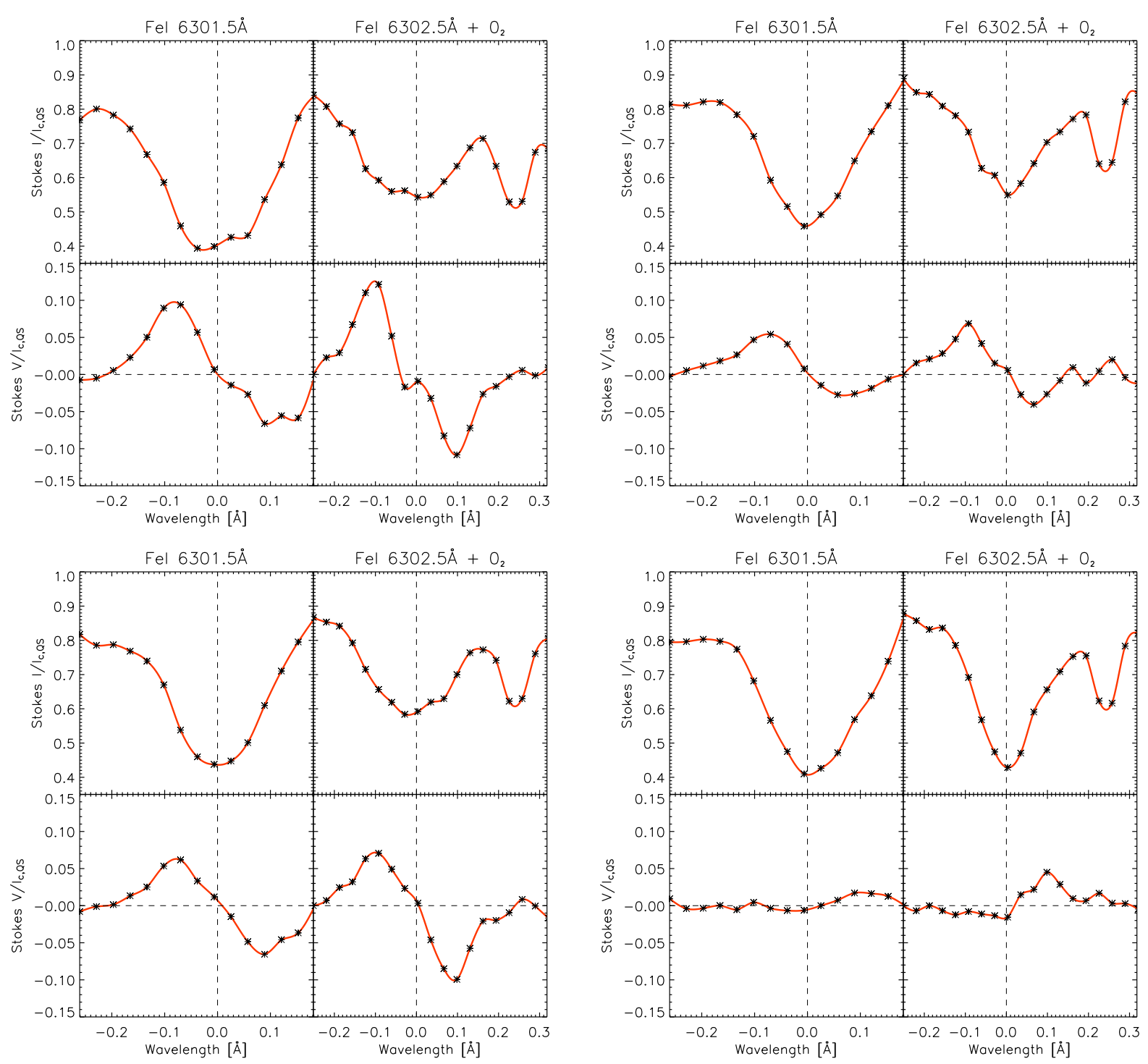

Figure 5.2: Examples of asymmetries in Stokes $I$ and $V$ profiles of the Fe I lines at $6302 \AA$ from the limb-side penumbra.Upper left panels represent profiles for a penumbral grain close to the umbra-penumbra border, coordinates $(x, y) \sim\left(11^{\prime \prime}, 10^{\prime \prime} 5\right)$ in the broad-band reconstructed images (Fig.4.1 lower panel, left). Upper right panels correspond to a dark region in the very outer penumbral border, coordinates $(x, y) \sim\left(5^{\prime \prime}, 14^{\prime \prime}\right)$. Lower left panels show profiles from a dark filament in between to bright filaments the mid penumbra, coordinates $(x, y) \sim\left(7^{\prime \prime}, 5^{\prime \prime}\right)$. Lower right panels show profiles from a intermediate region between a bright and a dark filament in the mid-outer penumbra, coordinates $(x, y) \sim\left(7 ! 5,12^{\prime \prime}\right)$. Red curves are interpolation to the observed scanning-positions (stars) along the spectral lines. Vertical lines represent the wavelength reference, i.e. line centre of the averaged Stokes $I$ profiles in the surrounding photosphere. The narrow spectral feature on the red side of $\lambda 6302.5 \AA$ (at $\Delta \lambda \approx 0.25 \AA$ from the line centre) is a telluric $\mathrm{O}_{2}$ line.

One notices the big amount of asymmetries which can be found in the penumbral Stokes profiles. The examples exposed here are only a small sample. One notices especially the last two examples shown in Fig.5.3. They both correspond to a region in 

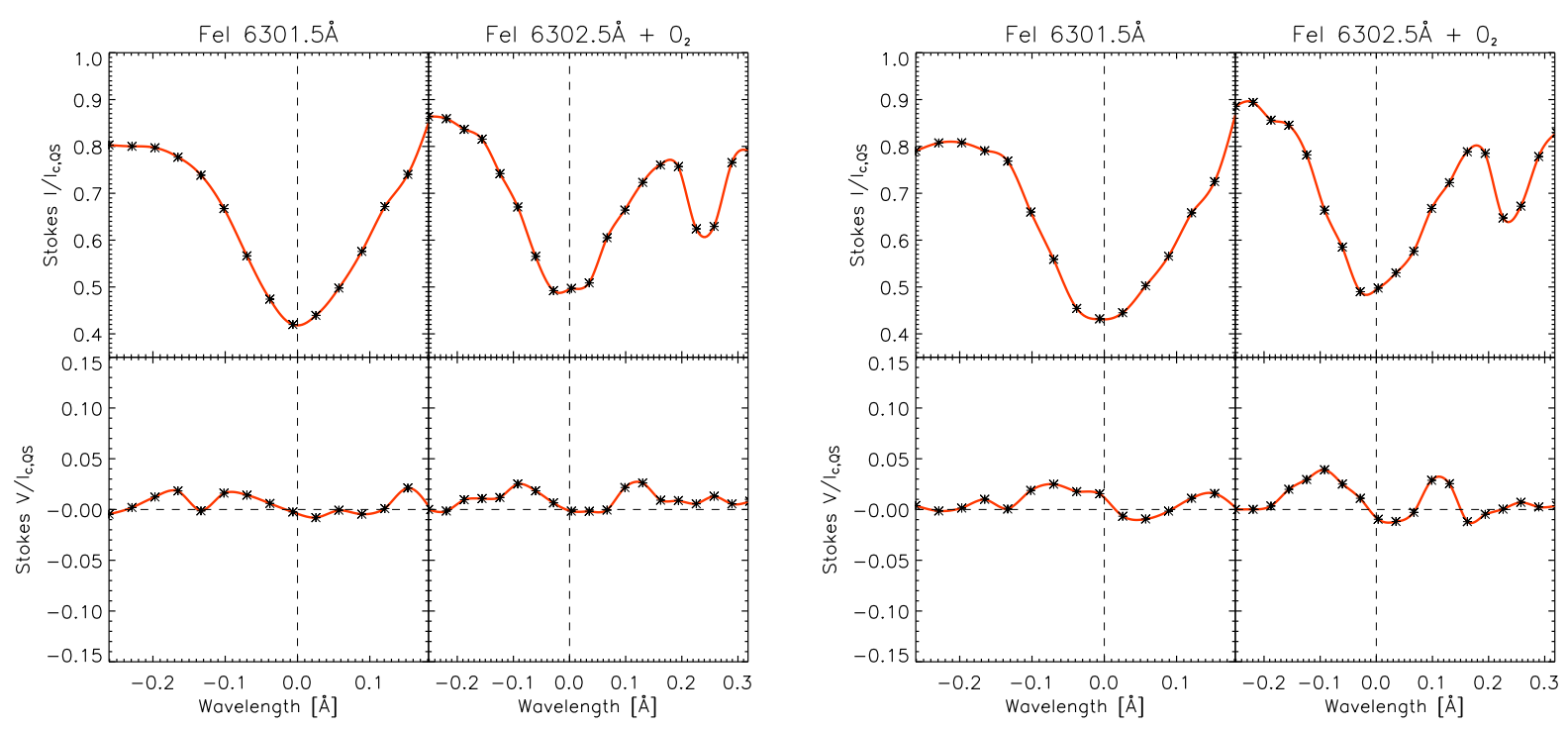

Figure 5.3: Examples of asymmetries in Stokes $I$ and $V$ profiles of the Fe I lines at $6302 \AA$ from the limb-side penumbra. Panels show profiles from intermediate positions between dark and bright filaments in the mid penumbra, coordinates $(x, y) \sim\left(77^{\prime \prime} 5,12^{\prime \prime}\right)$ and $(x, y) \sim\left(6{ }^{\prime \prime} .5,11^{\prime \prime}, 5\right)$, respectively. Red curves are interpolation to the observed scanning-positions (stars) along the spectral lines. Vertical lines represent the wavelength reference, i.e. line centre of the averaged Stokes $I$ profiles in the surrounding photosphere. The narrow spectral feature on the red side of $\lambda 6302.5 \AA$ (at $\Delta \lambda \approx 0.25 \AA$ from the line centre) is a telluric $\mathrm{O}_{2}$ line.

the limb-side mid penumbra of coordinates $(x, y) \sim\left(7^{\prime \prime} .5,12^{\prime \prime}\right)$ and $(x, y) \sim\left(6^{\prime \prime} .5,11^{\prime \prime} .5\right)$ in the broad-band reconstructed images (Fig.4.1 lower panel, left), respectively. The Stokes $V$ profiles, although weak, show clear uncommon shapes. Such Stokes $V$ profiles may be formed close to the magnetic neutral line, i.e. the virtual line along the limb-side penumbra where more vertical magnetic fields, bending into more horizontal, reach the perpendicular direction to the LOS. The anomalously shaped profiles observed there, as those reported by Sánchez Almeida \& Lites (1992), among others, may be the composition of Stokes $V$ with different polarity, a phenomenon originally called as crossover effect (Grigorjev \& Katz 1972).

Understanding the origin of the asymmetries in the Stokes profiles coming from a given structure means to understand how magnetic and velocity fields are stratified in the atmosphere along the line-of-sight in such structure. Radiative transfer calculations by assuming adequate atmosphere models are a useful tool to analyse these asymmetries. Different numerical methods can be used. Many authors apply the popular inversion techniques (e.g. Bellot Rubio et al. 2004, Borrero et al. 2004, Sánchez Almeida 2005) consisting in the retrieve of the atmospheric parameters which best fit the synthetic profiles to the observed ones, starting from an initial input atmosphere model. Another possibility is to apply forward modelling, i.e. to develop a semi-empirical model controlled by various parameters (temperature, magnetic field, geometry of the problem, ...). By varying the input parameters, one retrieves different synthetic Stokes profiles that can be compared with the observed ones. 

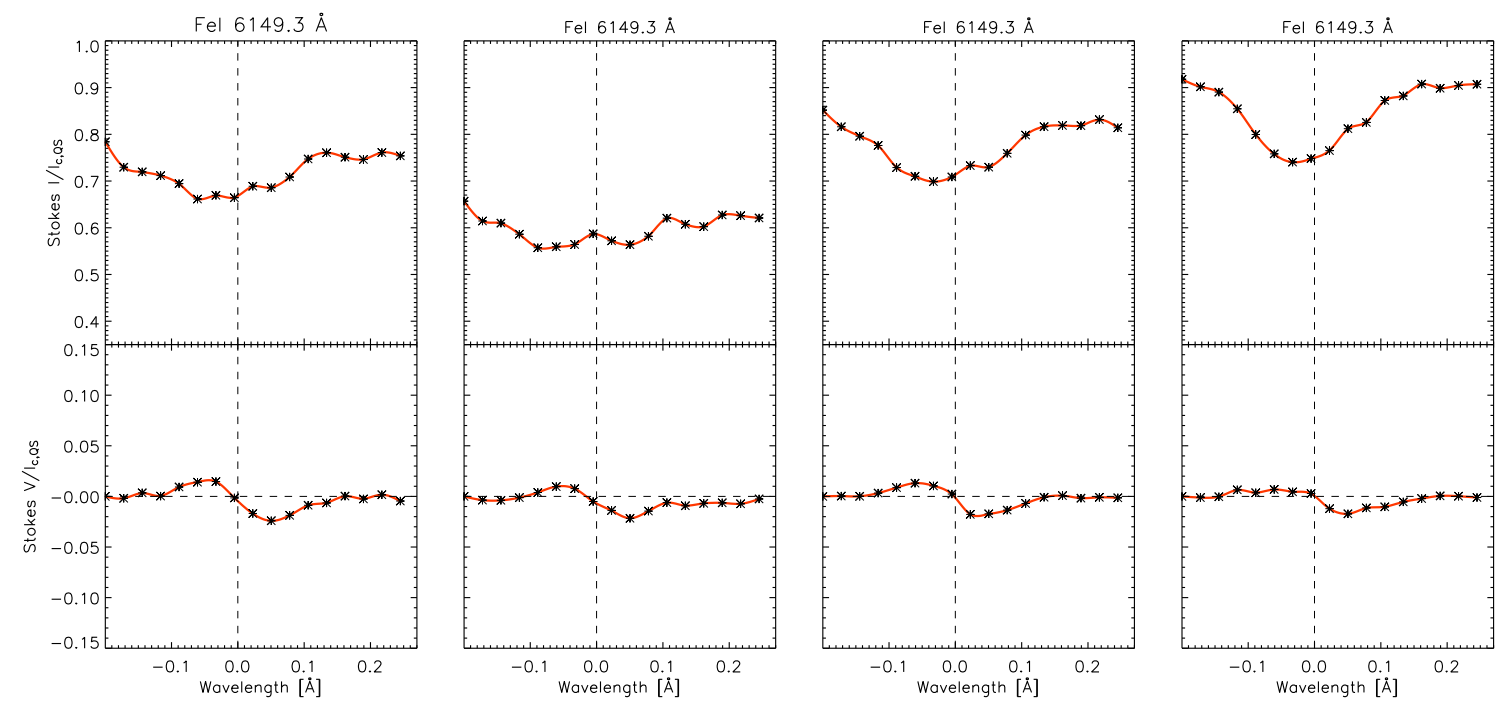

Figure 5.4: Examples of asymmetries in Stokes $I$ and $V$ profiles of the Fe II $6149.3 \AA$ line, from the centre-side penumbra. To reduce noise, profiles averaged over some area of specific penumbral structures are presented. From left to right: first two panels are related to two penumbral grains near the umbra-penumbra border with coordinates in the broad-band images from Fig.4.1 (upper, right) of, $(x, y) \sim\left(2^{\prime \prime}, 13^{\prime \prime}\right)$ and $(x, y) \sim\left(3^{\prime \prime}, 10^{\prime \prime}\right)$, respectively; last two panels from a bright head in the mid penumbra at $(x, y) \sim\left(4^{\prime \prime}, 7^{\prime \prime}\right.$.5) and a bright head closer to the outer penumbral border at $(x, y) \sim\left(6^{\prime \prime}, 4^{\prime \prime}\right)$. Red curves are the interpolation to the observed scanning-positions (stars) along the spectral line. Vertical lines represent the wavelength reference, i.e. line centre of the averaged Stokes $I$ profiles in the surrounding photosphere.
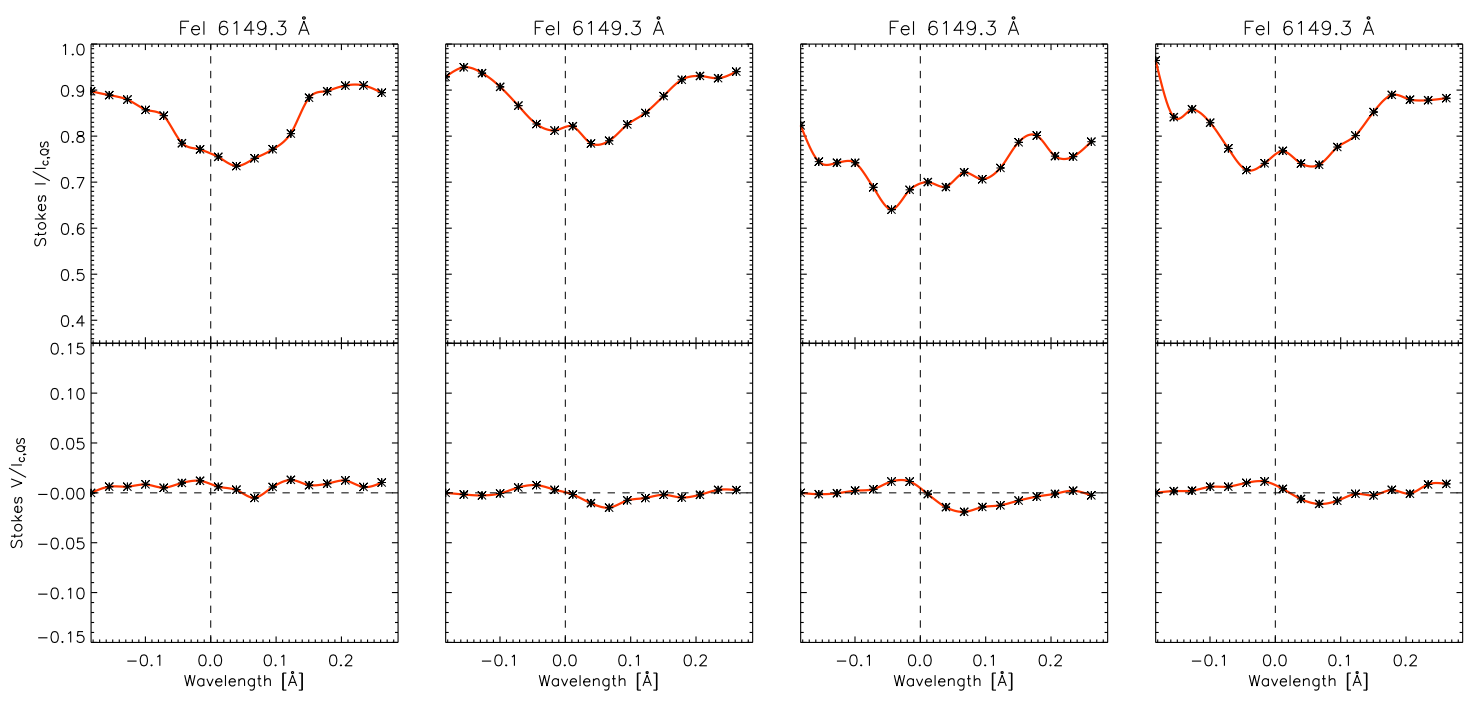

Figure 5.5: Examples of asymmetries in Stokes $I$ and $V$ profiles of the Fe II $6149.3 \AA$ line, from the limb-side penumbra. Averaged profiles are represented, as in Fig.5.4 From left to right: a dark structure in between two bright filaments in the mid penumbra, with coordinates in the broad-band images from Fig. 4.1 (upper, left), $(x, y) \sim\left(9^{\prime \prime} .5,4^{\prime \prime}\right)$; the connection between two visible bright heads at $(x, y) \sim\left(10^{\prime \prime}, 6^{\prime \prime}\right)$; last to panels correspond to the heads of two different filaments intruding into the umbra at $(x, y) \sim\left(11^{\prime \prime} .5,7^{\prime \prime} \cdot 5\right)$ and $(x, y) \sim\left(10^{\prime} \cdot 5,11^{\prime \prime}\right)$, respectively. 


\section{Effect of crosstalk on the observed asymmetries}

One may think that the asymmetries shown by the observed Stokes profiles are the result of crosstalk effects. It has been already dicussed in Sec.3.2.4 that the contribution of crosstalk from $Q, U, V \rightarrow I$ is negligible. About the effect of crosstalk from $Q$, $U \rightarrow V$, an underestimation of $20 \%$, at most, on the LOS magnetic field using the COG method was found. Now, the effect of the crosstalk on the asymmetries shown by the Stokes $V$ profiles is quantified by comparing synthetic profiles unaffected and affected by crosstalk. The profiles are calculated for a two-component dynamical atmosphere aiming to have more asymmetric profiles. The profiles are presented in Fig.5.6 in a sequence calculated for different inclination angles (of the velocity and the magnetic field) for the background component. The blue curves represent $V$ profiles affected by crosstalk. The crosstalk contribution increases with the inclination angle (with respect to the vertical) as expected from the increasing stronger contribution from the transverse Zeeman effect. The difference between affected and unaffected crosstalk is estimated to a maximum value of $0.021 I_{c}$ (continuum intensity).
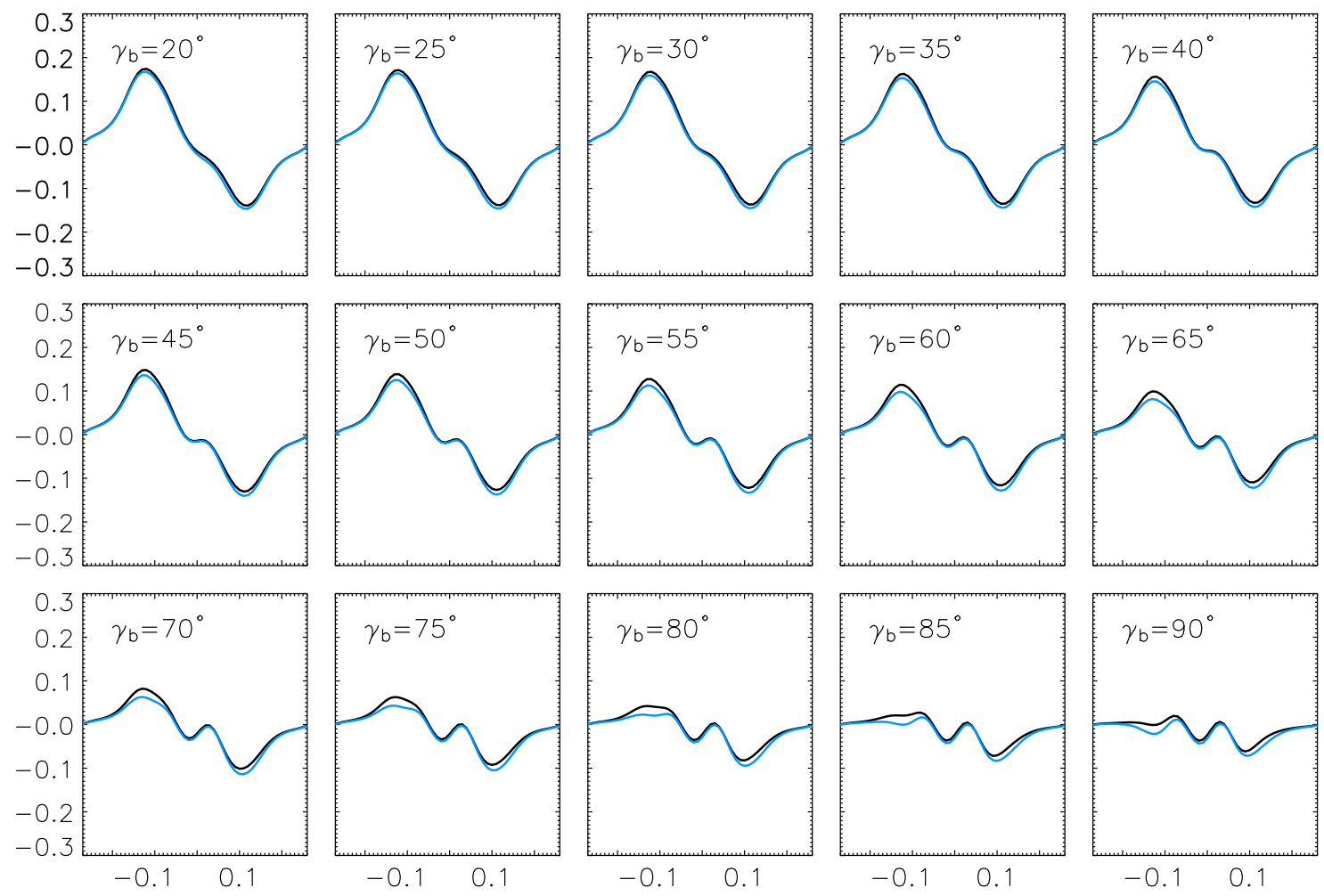
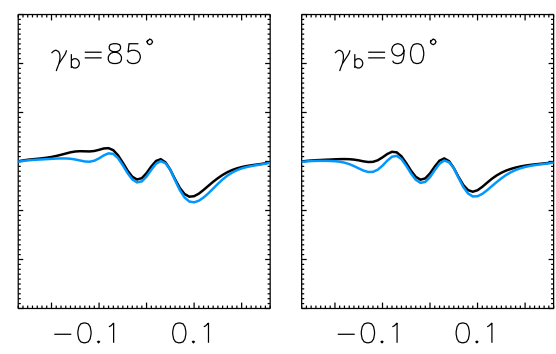

Figure 5.6: Effect of the crosstalk on the Stokes $V$ profiles with variation of the inclination angle $\gamma_{b}$. Curves in blue show synthetic Stokes $V$ profiles 'affected' by crosstalk effects. They can be compared with synthetic $V$ profiles without crosstalk (in black). The $\gamma_{b}$ angle is the inclination of the background atmosphere component. The profiles were calculated for a dynamic two-component atmosphere as described in Sec.5.2.2

The worst case of crosstalk effect has been taken into account in the above mentioned estimations. The real contamination can be smaller. Thus, the main contribution to the observed asymmetries are in any case of solar origin. 


\subsection{Two-component penumbral model}

The area asymmetry is a parameter used by many authors to account for the asymmetries observed in the Stokes $V$ profiles and therefore to account for variations in the magnetic and the velocity field along the line-of-sight (LOS). It is defined as

$$
\delta A=\frac{\int V(\lambda) \mathrm{d} \lambda}{\int|V(\lambda)| \mathrm{d} \lambda} .
$$

Sánchez Almeida \& Lites (1992) found that in order to reproduce the large area asymmetry observed in penumbrae in the Fe I lines at $6302 \AA$, gradients for LOS velocities as large as $\left|\frac{\mathrm{d} v_{L O S}}{\mathrm{~d} \tau}\right| \sim 1.5 \mathrm{~km} \mathrm{~s}^{-1}$ and $\left|\frac{\mathrm{d} \gamma}{\mathrm{d} \tau}\right| \sim 45^{\circ}$ for the magnetic field inclination with respect to the LOS were needed. Solanki \& Montavon (1993) proposed the uncombed penumbral model, i.e. a horizontal magnetic flux tube embedded in a more vertical background field, in order to introduce the strong gradients needed to reproduce the observed area asymmetry. Later, Schlichenmaier et al. (2002) and Müller et al. (2002) pointed out that the area asymmetry observed in the visible lines could be dominated by jumps in the magnetic field inclination.

Following those previous works, the atmospheric model considered in this Thesis is based on the assumption that our resolution element contains two different components corresponding to different atmospheres along the LOS. The scenario may be described as follows:

- One component is considered as a static background atmosphere characterised by a mean penumbral model by del Toro Iniesta et al. (1994), a typical magnetic field inclination of $45^{\circ}$ and magnetic field strength $B_{\text {back }}$ as a function of the optical depth at $\lambda 5000 \AA$ as proposed by Martínez Pillet (2000), and

- a second component given by a 'flux tube' embedded in the background atmosphere with magnetic field strength $B_{\text {tube }}<B_{\text {back }}$. Velocities and magnetic field inclination follow the geometry of the tube which is in lateral pressure balance with the background:

$$
P_{\text {tube }}+\frac{B_{\text {tube }}^{2}}{8 \pi}=P_{b a c k}+\frac{B_{b a c k}^{2}}{8 \pi}
$$

where $P_{\text {tube }}$ and $P_{\text {back }}$ are the pressure composed of gas pressure and 'microturbulent' pressure (cf. Eq.5.6, below) inside the flux tube and in the background medium, respectively.

\subsubsection{Geometry of the model}

The geometry of the flux tube as shown in Fig.5.7 represents a first approximation of the widely used picture of the penumbral fine-structure as consisting of small flux tubes embedded in a magnetic background atmosphere. The flux tube carries the Evershed flow. Hot gas emerges almost vertically from deep layers. Then, the flux tube and the velocity turn into a more horizontal direction. In this horizontal flow the gas cools down. 
And finally the flow carrying cooler material submerges into sub-photospheric layers. Emergence and submerging can repeat as proposed in the picture of the sea serpent by Schlichenmaier et al. (2002). Thus, the geometry of the flux tube, with typical diameter of $\sim 100 \mathrm{~km}$, is divided into three scenarios (cf. Fig. 5.7).

- upflow with temperature $T_{\text {tube }}>T_{b a c k}$,

- horizontal flow with temperature $T_{\text {tube }}<T_{\text {back }}$,

- downflow with temperature $T_{\text {tube }}<T_{\text {back }}$.

Our study is focused on the case of a heliocentric angle of $\theta=20^{\circ}$ which corresponds approximately to the position on the solar disc of the observed sunspots. This angle is taken into account to calculate the LOS components of the vector fields and in such a way that one can differentiate between 'observing' a centre-side and a limb-side modelled penumbral atmosphere.

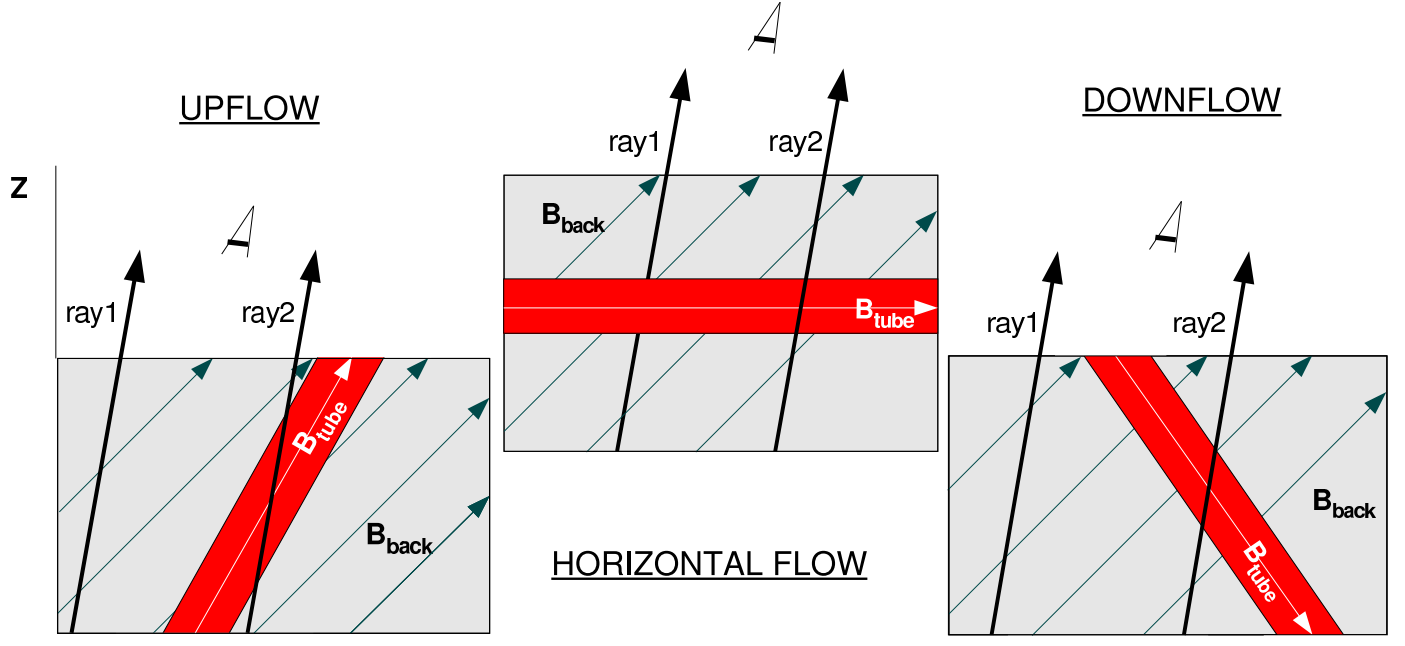

Figure 5.7: Scheme of the geometry of the model as seen from the 'centre side'.

\subsubsection{Atmospheric parameters for the one-component model}

In order to solve the RTE for the main model based on only one component ('background'), the physical quantities as functions of the optical depth - temperature $T(\tau)$, magnetic field strength $B(\tau)$, magnetic field inclination with respect to the vertical $\gamma(\tau)$, magnetic field azimuth $\phi(\tau)$, line-of-sight velocities $v_{L O S}$ and microturbulent velocity $\xi_{\text {mic }}(\tau)$ - are needed. The atmosphere code for the computation of the atmospheric absorption properties, originally developed at the Universitäts-Sternwarte Göttingen, has as initial input parameters the temperature $\left(T=T_{e}\right)$, the column mass density $(m)$ and the microturbulence velocity $\left(\xi_{m i c}\right)$. The computational procedure can be briefly described as follows: 
- The gas is mainly composed of the elements $\mathrm{H}, \mathrm{He}, \mathrm{C}, \mathrm{O}, \mathrm{Na}, \mathrm{Mg}, \mathrm{Al}, \mathrm{Si}, \mathrm{S}$ and $\mathrm{Fe}$, with standard solar abundances, of the ions of these elements and of electrons. The list contains also $\mathrm{Na}$ since it contributes, due to its low ionisation potential (see Table 5.17, also non-negligibly to the electron density.

\begin{tabular}{lccc}
\hline $\mathrm{El}$ & $\mathrm{A}_{E}$ & $\mathrm{E}_{i}$ & $\mathrm{E}_{e}$ \\
\hline $\mathrm{H}$ & 1.000 & 13.59 & - \\
$\mathrm{He}$ & 0.080 & 24.58 & 54.40 \\
$\mathrm{C}$ & $3.55 \times 10^{-4}$ & 11.26 & 24.38 \\
$\mathrm{O}$ & $5.90 \times 10^{-4}$ & 13.61 & 35.11 \\
$\mathrm{Na}$ & $1.51 \times 10^{-6}$ & 5.14 & 47.29 \\
$\mathrm{Mg}$ & $3.02 \times 10^{-5}$ & 7.64 & 15.03 \\
$\mathrm{Al}$ & $2.51 \times 10^{-6}$ & 5.98 & 18.82 \\
$\mathrm{Si}$ & $3.55 \times 10^{-5}$ & 8.15 & 16.34 \\
$\mathrm{~S}$ & $1.62 \times 10^{-5}$ & 10.36 & 23.40 \\
$\mathrm{Fe}$ & $3.16 \times 10^{-5}$ & 7.83 & 16.18 \\
\hline
\end{tabular}

Table 5.1: Abundances and ionisation energies for major elements. The abundances $A_{E} \equiv$ $N_{e l} / N_{H}$ have been taken from HSRA (Gingerich et al. 1971) with minor modifications according to more recent determinations. The energies $\mathrm{E}_{i}$ and $\mathrm{E}_{e}$ are in $e V$ and measure the ionisation for the neutral and singly-ionised stage, respectively.

- To compute the particle number densities for all species (atoms and ions of all considered elements), the electron pressure $P_{e}=N_{e} k T$ (where $N_{e}$ is the electron number density) is needed. Since $P_{e}$ is itself a function of the particle number density, the problem must be solved iteratively. For this we assume hydrostatic equilibrium

$$
\mathrm{d} P_{t o t}=g \mathrm{~d} m
$$

which can be integrated to yield

$$
P_{\text {tot }}=g m+P_{0}
$$

or, with the implicit function of $P_{e}$

$$
F\left(P_{e}, T\right)=P_{t o t}\left(P_{e}, T\right)-g m-P_{0}=0 .
$$

Here, $g$ is the surface gravity, $P_{0}$ is the pressure at the top of the atmosphere, i.e. in the corona $\left(P_{0} \approx 0\right)$. The total pressure is given by

$$
P_{\text {tot }}=P_{\text {gas }}+\frac{1}{2} \rho \xi_{m i c}^{2}
$$

where $P_{\text {gas }}$ is the gas pressure, $\frac{1}{2} \rho \xi_{m i c}^{2}$ the pressure term associated with the microturbulence, $\xi_{m i c}$, and $\rho$ the mass density. The gas pressure and the mass density are calculated from an initial guess of $P_{e}$. Next, the electron pressure is iterated using Newton's secant method until the hydrostatic equilibrium is fulfilled to a required precision of $10^{-5}$. In other words, one seeks, from an initial guess of $P_{e}$, a zero of the implicit function $F\left(P_{e}, T\right)$, for a specific temperature $T$. 
- Once the mass density is known, the geometric height, $z$, is obtained from the relation

$$
\mathrm{d} m=-\rho \mathrm{d} z .
$$

- The estimate of the continuum opacity or continuum absorption coefficient, $\kappa_{c}$, takes into account the contributions due to $\mathrm{H}^{-}$bound-free and free-free transitions as well as the small contributions from the $H$ free-free transitions and from the Paschen-, Bracket- and Pfund bound-free transitions of hydrogen, as explained in Pérez Rodríguez \& Kneer (2002).

- The number density of absorbers is calculated for each spectral line under consideration, combining the Saha and Boltzmann equations (Eq.2.25] and Eq.2.26). They are calculated using the solar abundance of the elements of each spectral line, the lower excitation energy, the partition functions of the relevant ionisation states, the collisional damping and the oscillator strengths. The relevant values for the iron line pair at $6302 \AA$ and for the ionised iron line at $6149.3 \AA$ are given in Table 5.2

- The components of the Zeeman pattern and their splitting are calculated for each layer from the electronic configuration of the transition for each spectral line and the magnetic field strength, $B$, which are input parameters given to the code.

- The absorption and dispersion profiles are evaluated at each layer for each wavelength in the spectral line, taking into account the appropriate Zeeman splitting, Doppler shifts, Doppler broadening and damping parameters.

- Thus, the absorption or propagation matrix can be calculated for each optical depth $\tau$, i.e. for each atmospheric layer. Finally, the RTE is integrated using the DELO method described in Sec.2.4.1.

\section{Initial one-component model}

As initial input parameters used for the 'background' component of the modelled atmosphere, the mean penumbral model by del Toro Iniesta et al. (1994) has been used. Other input values needed to characterise the model atmosphere are:

- the atomic data 1 of the spectral lines to synthesise (Table 5.2 );

- the wavelength grid;

- the heliocentric angle. It has been fixed to $20^{\circ}$ corresponding approximatly to the heliocentric angle of NOOA $9919\left(\theta=20^{\circ}\right)$ and NOAA $0346\left(\theta=23^{\circ}\right)$ during observations;

- the macroscopic broadening velocity, whose effect is introduced by convolving the profiles with its associated Gaussian function;

\footnotetext{
${ }^{1}$ The data (Table5.2 have been taken from the compilation of the values available in the online databases: Kurucz Atomic Line Database, NIST Atomic Spectra Database and the Vienna Atomic Line Data-Base.
} 
- LOS velocities and magnetic field strength, inclination and azimuth. Their values can be adjusted according to the asymmetries one wants to reproduce.

\begin{tabular}{llllllll}
\hline \hline$\lambda[\AA]$ & $Z$ & $m_{A}$ & $\chi_{i}[\mathrm{eV}]$ & $\chi_{e}[\mathrm{eV}]$ & $\log \left(g_{w} f\right)$ & $\Gamma$ & $J, L, S-J^{\prime}, L^{\prime}, S^{\prime}$ \\
\hline \hline & & & & & & & \\
6149.258 & 26 & 55.85 & 7.87 & 3.65 & -2.904 & -7.94 & $0.5|2| 1.5-0.5|1| 1.5$ \\
6301.498 & 26 & 55.85 & 7.87 & 3.65 & -0.745 & -7.62 & $2|1| 2-2|2| 2$ \\
6302.494 & 26 & 55.85 & 7.87 & 3.69 & -1.203 & -7.62 & $1|1| 2-0|2| 2$ \\
\hline \hline
\end{tabular}

Table 5.2: Input atomic values in the synthesis of the given spectral lines: wavelength, atomic number $(Z)$, atomic mass $\left(m_{A}\right)$, the ionisation potential $\left(\chi_{i}\right)$, the excitation potential $\left(\chi_{e}\right)$, the oscillator strength contribution (with $g_{w}$ the statistical weight of the lower level and $f$ the oscillator strength), the van der Waals damping constant $(\Gamma)$ and the quantum numbers characteristic of the transition (lower and the upper energy levels, respectively).

\section{Atomic parameters}

Often the atomic parameters found in the literature are not very accurate. Therefore the oscillator strengths and damping parameters of the transitions were tuned by test calculations which served also as parts of tests of the code. For that purpose, Stokes I profiles synthesised for the VALC quiet-Sun atmospheric model (Vernazza et al. 1981) are compared with those from the Fourier Transform Spectrometer Atlas by Brault \& Neckel (cited by Neckel, 1999). Similar to Pérez Rodríguez \& Kneer (2002), in order to obtain a better fit in the wings of the spectral line, the values of the damping constants had to be enhanced by a factor of approximately 5, compared to the prescription by Unsöld (1955). Results are shown in Fig.5.8
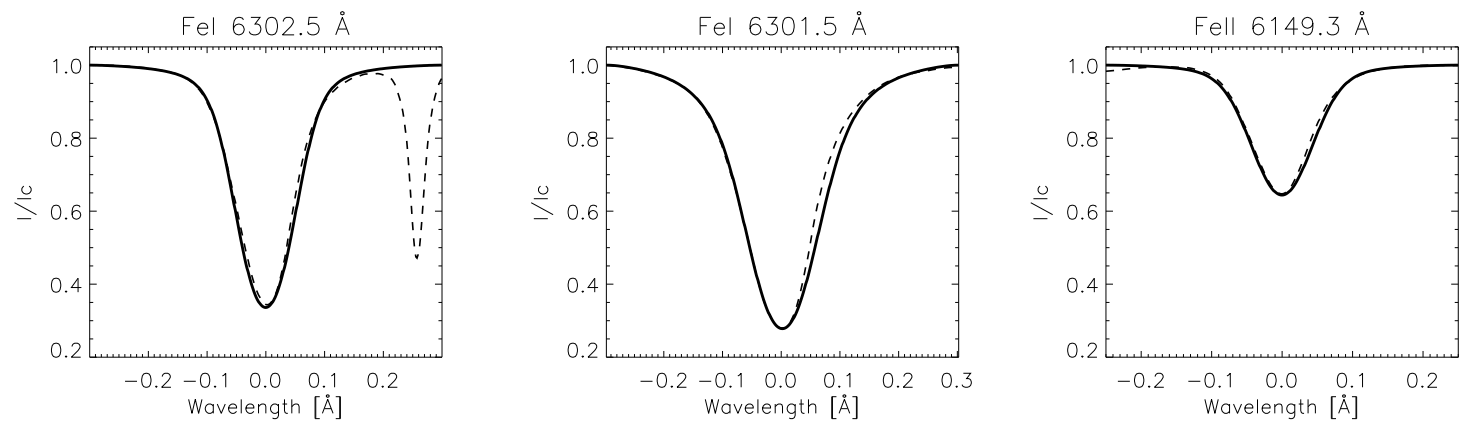

Figure 5.8: Comparison between synthetic intensity profiles (solid), calculated for the quiet Sun VALC model (Vernazza et al. 1981), and observed lines from the Fourier Transform Spectrometer Atlas (dashed). 


\section{Two-component model}

When the second component or flux tube is introduced in the model, the procedure consists in integrating the RTE for two independent rays: one 'crossing' exclusively the background atmosphere, ray 1 , and a second ray crossing both the background and the tube atmospheres, ray 2 (see Fig.5.7). For the second ray, the atmospheric parameters are calculated in a similar way as for those of the background component, but taking into account its different temperature stratification, velocity and magnetic field as well as the geometrical specifications of the tube (radius, height in the atmosphere, ...). The lateral pressure balance (Eq.5.2) is required for the calculation of the gas pressure, for which the iterative method described above is also used. The final synthetic Stokes vector $\left(I_{\text {syn }}\right)$ is obtained from the linear combination of the synthetic profiles calculated separately for ray 1 and ray 2 and weighted by a filling factor $(f)$, a further input parameter in the code. Thus, it follows

$$
I_{\text {syn }}=(1-f) I_{\text {ray } 1}+f I_{\text {ray } 2} .
$$

It is important to remark that the geometry of the two-component atmosphere so far described, i.e. the magnetic flux component embedded in a magnetic less inclined component in three different scenarios, corresponds to the initial idea the code was developed for. Thus, all input parameters, e.g. inclination of the background and the flux tube component, magnetic field strengths, velocities, filling factor, radius of the tube, height of the tube in the atmosphere, etc., are all free parameters which can be, and indeed they are, modified by the user aiming to simulate the observed profiles. Obviously, typical values found in the literature (e.g. Martínez Pillet 2000, Borrero 2004) for inclination angles, radius of the tubes, etc., are taken into account to reduce the big amount of output models.

\section{Reference wavelength}

For comparison of the observed and simulated profiles, the reference wavelength is important. We compared the sunspot line positions with those of the average profiles observed in the ambient quiet photosphere. Then the convective blueshif 2 of the latter has been taken into account. It amounts, according to Schröter (1956), to $\delta \lambda \sim 8 \mathrm{~m} \AA$ at these wavelengths and for a heliocentric angle of $20^{\circ}$.

\footnotetext{
${ }^{2}$ This blueshift of average quiet Sun profiles is caused by different intensity contributions from bright upflowing granules and dark downflowing intergranules. Thus, only close to the limb of the Sun, the gravitational redshift required by General Relativity is measured.
} 


\subsection{Synthesis of Stokes profiles}

\subsubsection{Reproduction of the observed asymmetries}

\section{Non-expected scenario: Cool upflows}

It is found from the observed filtergrams that the darkest penumbral fibrils show a local continuum intensity of about 0.75 of the quiet-Sun continuum intensity $I_{c, Q S}$, about 0.1 below the mean penumbral intensity $\left(0.85\right.$ of $\left.I_{c, Q S}\right)$. When the synthesis of profiles is performed considering the scenario of an 'upflow' ( $30^{\circ}$ of inclination with respect to the vertical) with temperatures $T_{\text {tube }} \leq T_{\text {back }}$, and 'observed' from a viewing angle that will be referenced as 'centre-side', the continuum intensity of the spectral lines always achieve values below 0.7 of $I_{c, Q S}$, independently of the values of the magnetic field strength, velocity, inclination of the tube, thickness of the tube (if larger than $100 \mathrm{~km}$ ), .. Only when the contribution of the tube component (upflow) is neglected assuming a low filling factor, e.g. 0.1 , the continuum intensity of the synthetic profiles will increase above 0.75 of $I_{c, Q S}$. This scenario has to be ruled out since an almost vertical upflow, observed at a heliocentric angle of $\theta=20^{\circ}$ must have a strong contribution along the line-of-sight. Moreover, synthetic profiles achieve a mean penumbral intensity value for the given geometry of the model and with a filling factor of $f=0.5$ (also found from inversions for the inner penumbra by Borrero, 2004) only when $\Delta T=T_{\text {tube }}-T_{\text {back }} \geq 1000^{\circ} \mathrm{K}$ (from inversions, Borrero (2004), finds $\Delta T \sim 500-900 K$ in upflows in the inner penumbra).
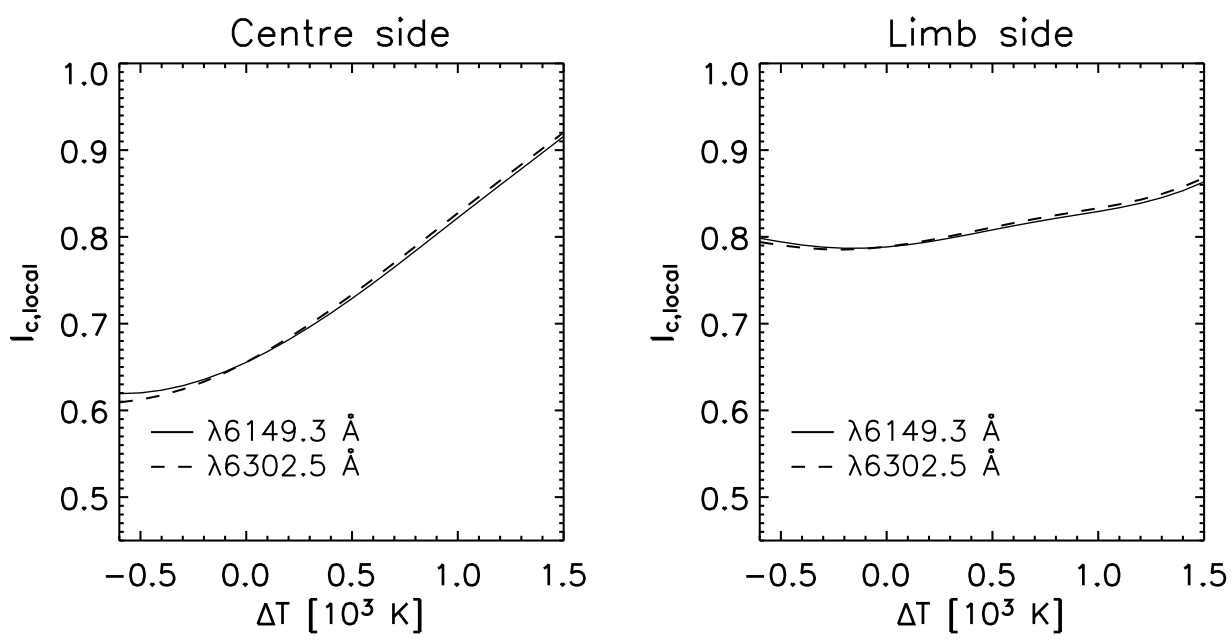

Figure 5.9: Representation of the variation of local continuum intensities $I_{c, l o c a l}$ of the indicated spectral lines with variations in the temperature of the tube, indicated by $T_{\text {tube }}=T_{\text {back }}+\Delta T$ where $T_{\text {back }}$ or temperature structure of the background is a fixed input. Left panel corresponds to values for the scenario of 'upflow' located at $z \sim 50-150 \mathrm{~km}$ and filling factor of 0.5 , observed at the centre-side penumbra and right for the same scenario at the limb-side penumbra,

However, when the viewing angle is such that one would refer to 'limb-side observations' (Fig.5.9 right), intensities from the local continuum of the spectral lines achieve 
characteristic penumbral values already at low temperature values in the tube. This must be due to projection effects. When the up-flowing tube is 'observed' on the centre side, the contribution along the line-of-sight is much larger than when the same tube is observed from an opposite angle. At low temperatures, the continuum opacity in the tube is low due to decreased electron density, thus decreased $H^{-}$density, and therefore more transparent. At the 'limb side' where the contribution of the tube is less, we might be observing mainly the hotter surrounding 'background' atmosphere, and therefore the intensity values are higher in this case. For the same reasons, when higher temperatures are considered, the tube becomes less transparent and therefore, in the centre side where the contribution of the tube is higher, larger intensities will be achieved. Therefore, it is plausible to conclude that upflows with temperatures $T_{\text {tube }} \leq T_{\text {back }}$ are not characteristic of the penumbral fine-structure.

\section{Penumbral grains}

Average Stokes profiles of a penumbral grain close to the umbra, structure with coordinates $(x, y) \sim\left(4^{\prime \prime}, 13^{\prime \prime}\right)$ in the intensity maps (e.g. Fig.4.1 lower panel, right), are shown in the lower four panels of Fig.5.10.
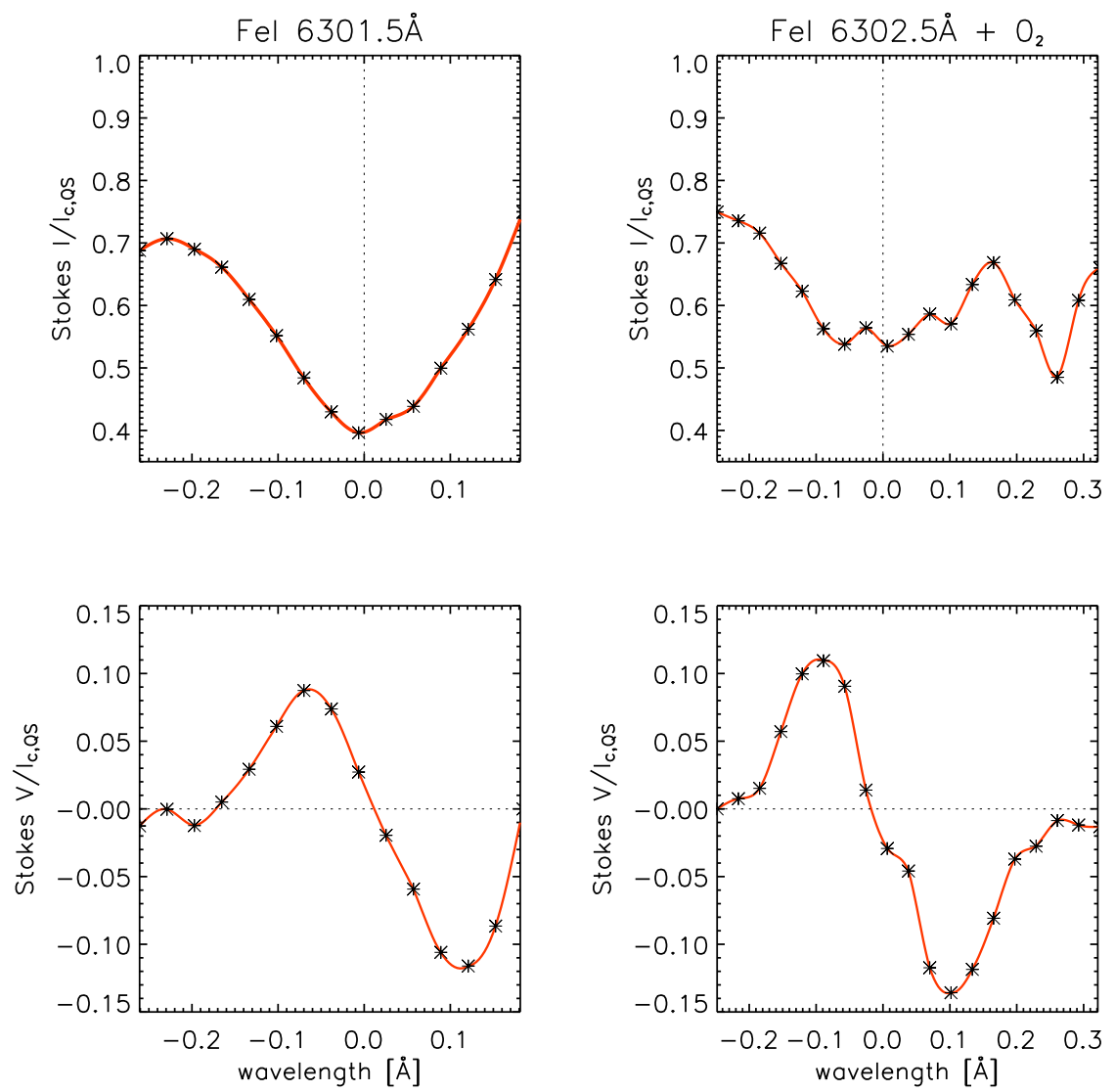

Figure 5.10: Stokes $I$ and $V$ profiles for $\lambda 6302.5 \AA$ and $\lambda 6301.5 \AA$, averaged over 0 "' $3 \times 0^{\prime \prime} .3$, from a penumbral grain in the umbra-penumbra border. 
The Stokes $I$ at $\lambda 6302.5 \AA$ shows clearly the two $\sigma$ and the $\pi$ components of the Zeeman pattern of this iron line. Moreover, observed profiles from other penumbral grains near the umbra all around the umbral-penumbral border show the triplet pattern in Stokes $I$ and a large signal in Stokes $V$ (cf. Fig.5.1 upper left and lower left panels). These are indications of a strong magnetic field and a strong component along the LOS in the structure and, since our sunspot is not far from the disc centre $\left(\theta \sim 20^{\circ}\right)$, these must correspond to a strong vertical component of the magnetic field.

It is important to note that the strong observed asymmetries cannot be produced by instrumental crosstalk, as it was explained above. They are inherent properties of the emergent penumbral profiles and are produced by penumbral dynamic processes.

The forward modelling procedure allows to fix some parameters like the geometry of the model, to vary other parameters like magnetic field strengths and velocities and to sample the atmosphere with as many grid points as needed. The latter possibility is important when one assumes a discontinuity along the ray as in the present modelling.
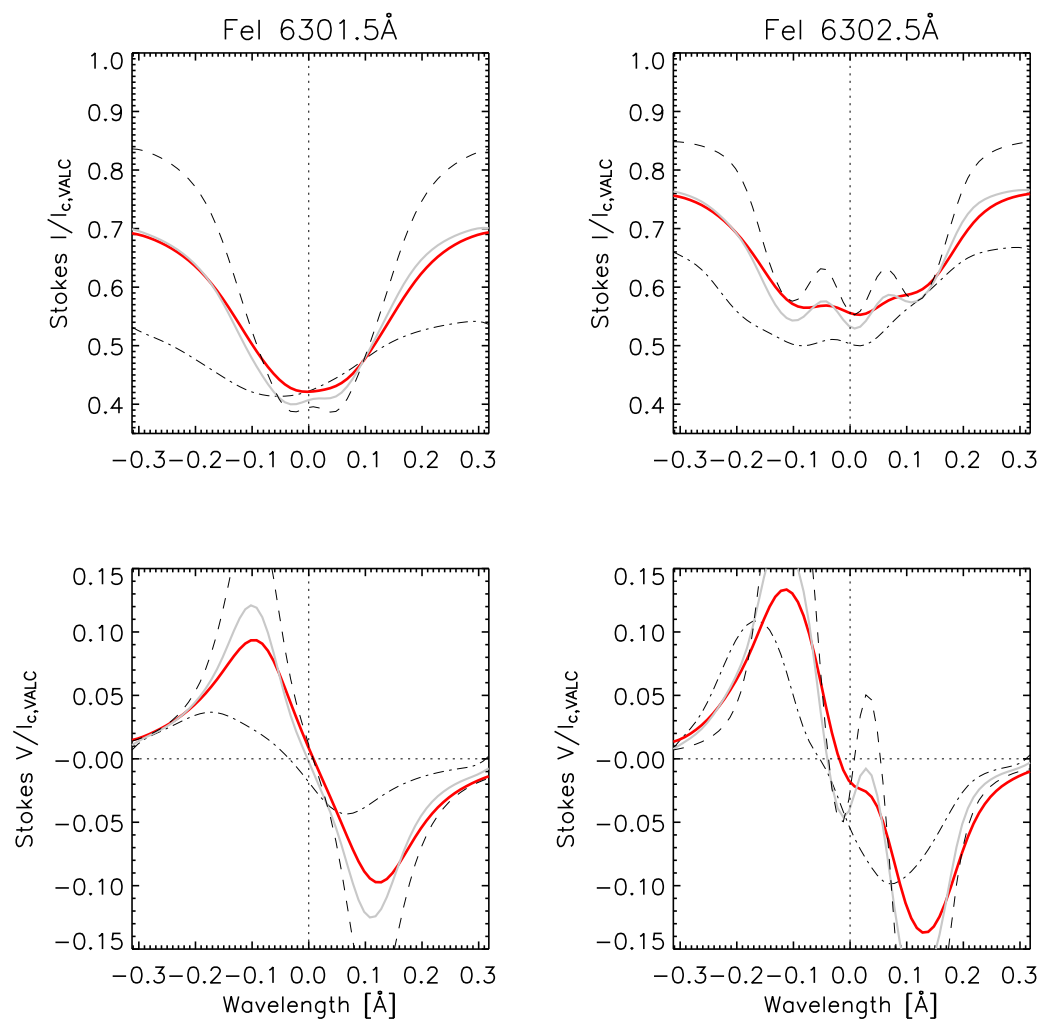

Figure 5.11: Examples of synthetic Stokes $I$ and $V$ profiles for $\lambda 6302.5 \AA$ and $\lambda 6301.5 \AA$. The dashed curves represent profiles synthesised from the ray crossing the 'background' component, $I_{\text {ray } 1}$, and the dashed-dotted curves the profiles from the ray crossing both components, $I_{\text {ray } 2}$. The grey solid curves represent the combination of the previous ones weighted with the filling factor. The red solid profiles are the result of the convolution of the latter profiles with the Airy function characteristic of the spectrometer used in the observations. For comparison with the observed profiles in Fig. 5.10 note the different wavelength range here.

Fig.5.11 shows two examples of synthetic profiles aiming at simulating the observed 
line asymmetries shown by the penumbral grain profiles from Fig.5.10 Asymmetries can be simulated by considering vertical upflows of material. The temperature in the tube was assumed higher than in the background as we expect from the picture of an upflow carrying hot material. The magnetic field strength, as a function of the optical depth, has values of $\sim 2500$ Gauss in deep layers for the background. The magnetic field strength for the tube is introduced by a step in the given background field of $\Delta B \sim-500$ Gauss. The absolute value of the velocity is as high as $10 \mathrm{~km} \mathrm{~s}^{-1}$ which gives a component along the LOS of 3-4 $\mathrm{km} \mathrm{s}^{-1}$ (see Fig. 5.12). The filling factor is about 0.5 and the heliocentric angle $\theta=+20^{\circ}$, meaning observations from the centre-side penumbra.
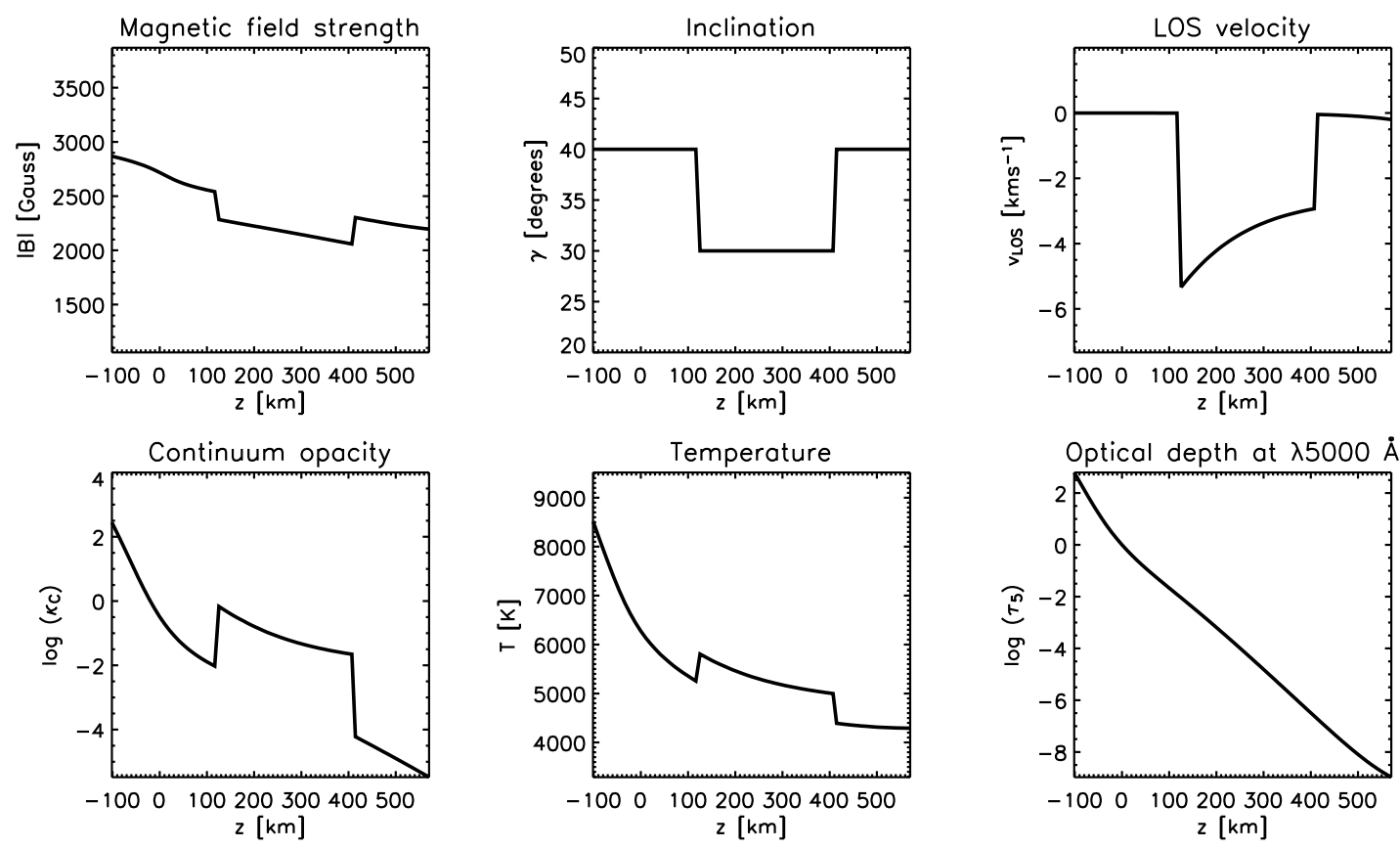

Figure 5.12: Atmosphere parameters along the integration path for the synthesis of the Stokes profiles shown in Fig.5.11

\section{Example of dark area in centre-side penumbra}

Another interesting example of the huge variety of phenomena occurring in the penumbral fine-structure is characterised by the profiles shown in Fig.5.13. These profiles also are the averages over an area of $0.3 \times 00^{\prime \prime} .3$ of the dark (relative to the local intensity) structure in the mid centre-side penumbra. The Stokes I profile of $\lambda 6302.5 \AA$ shows a quite distorted shape and therefore it has been selected aiming to reproduce comparable synthetic profiles.

The results are shown in Fig. ??. Although detailed comparison between observed and synthesised profiles reveals obvious differences, to arrive at simulating, after many simulating trials, the two components shown by the Stokes I profile of $\lambda 6302.5 \AA$ has only been possible by assuming a model with the following characteristics (see Fig. 5.15):

- heliocentric angle of $\theta=20^{\circ}$ from the centre-side viewing angle;

- initial velocity of the background component, $v_{b}=-0.2 \mathrm{~km} \mathrm{~s}^{-1}$, i.e. almost at rest; 

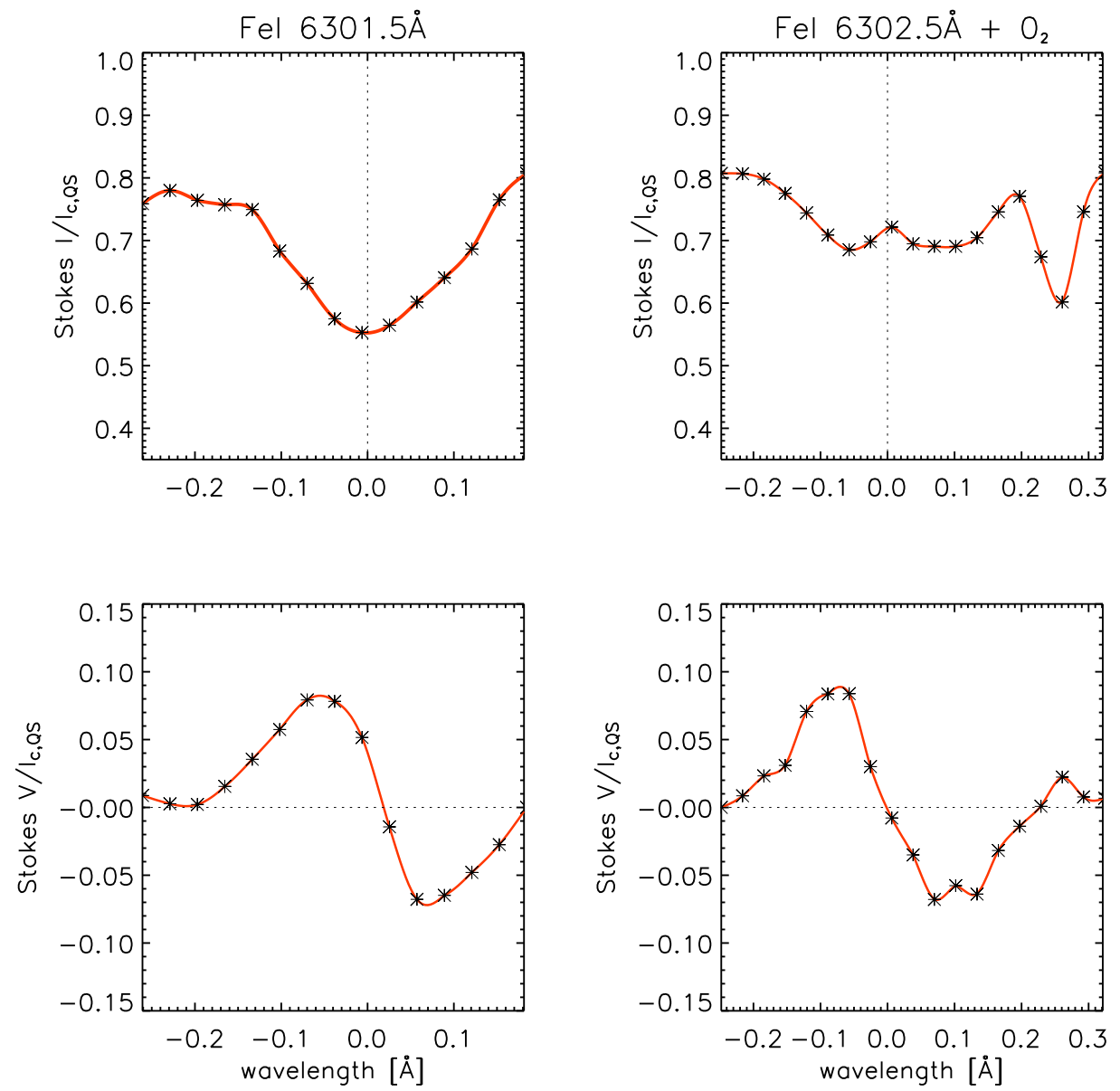

Figure 5.13: Example of temperature, magnetic field strength and LOS velocities along integration path for the two component model.

- inclination of the background component, i.e. inclination of the background magnetic and velocity fields, $\gamma_{b}=70^{\circ}$, i.e. nerarly horizontal;

- inclination of the flux tube component of $\gamma_{t}=-10^{\circ}$ with respect to the vertical, and therefore with the geometry, as shown in the third panel of Fig.5.7, $\gamma_{t}=-10^{\circ}$ indicates an in clination of $10^{\circ}$ with respect to the vertical but away from the LOS;

- LOS velocity of the flux component varying along the atmosphere with negative values, i.e. upflowing velocities;

- a diameter of the flux tube component of $d$ equivalent to $\sim 0$ '. 4 , therefore it should be considered as an upflow component rather than a 'tube' proper;

- the flux component, as it can be seen from the representation of the various parameters, starts at $\sim 100 \mathrm{~km}$ height in the atmosphere until higher layers which are out of the range of formation of the spectral lines used in the observations;

- moreover, a filling factor for the latter component of $f=0.8$ must be considered, which represents most of the contribution to the final profiles. 

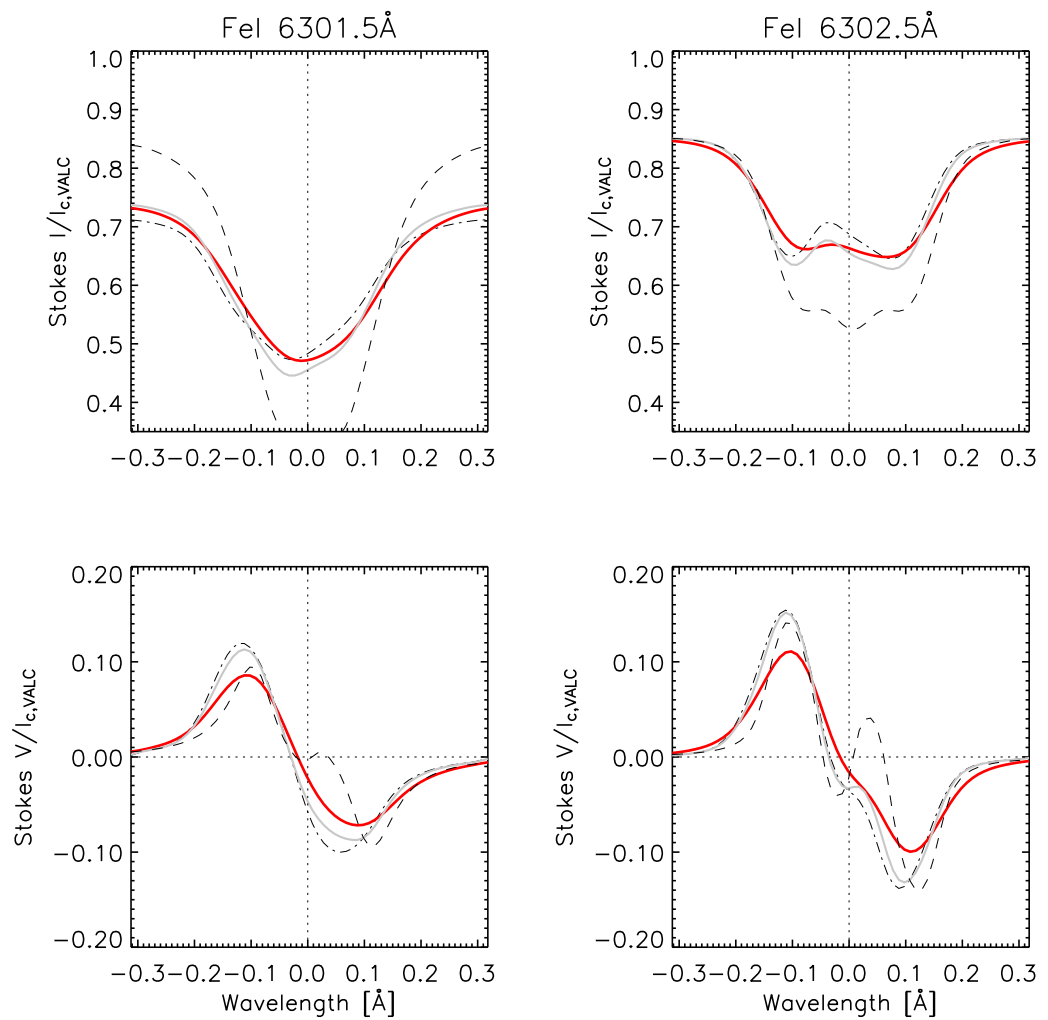

Figure 5.14: Synthetic Stokes $I$ and $V$ profiles for the Fe I line pair at $6302 \AA$. Curves are represented as in Fig 5.11
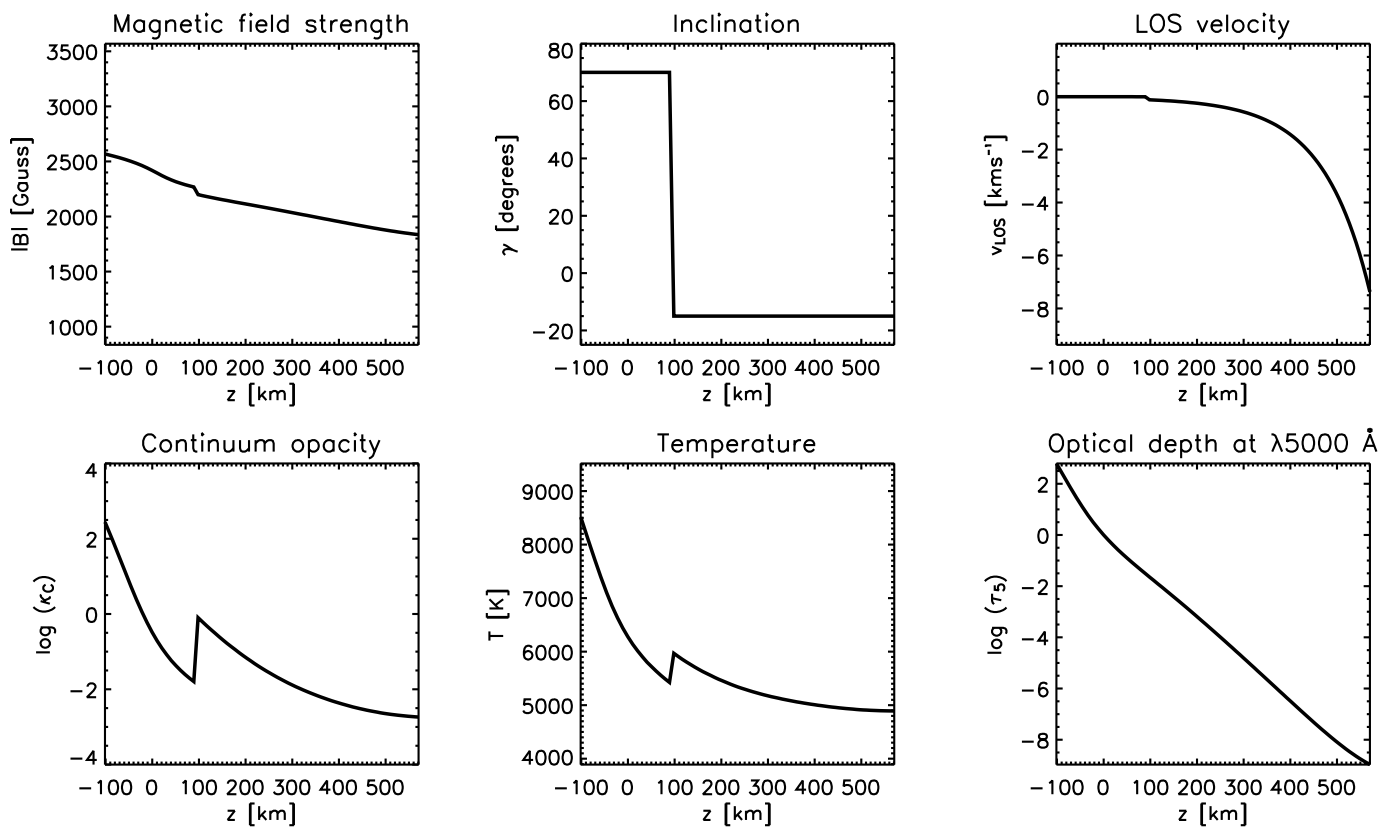

Figure 5.15: Parameter values associated to the synthesis of profiles shown in Fig. ?? as functions of the atmospheric height $z$. 


\subsubsection{Summary on the synthesis of Stokes profiles}

In the last sections, numerical modellings in the context of penumbral atmospheres have been presented. First, the procedure of calculating the various atmospheric parameters from the initial mean penumbral values of temperature, column mass and microturbulence velocity to solve the one-dimensional radiative transfer problem has been described. Next, the assumption of a more complex scenario to deal with the asymmetries shown by the observed Stokes profiles has been introduced. It was originally based on the idea of the uncombed penumbral model proposed by Solanki \& Montavon (1993), i.e. an atmosphere characterised by two different components. One component consists of a mean background penumbral atmosphere, originally considered to be at rest and with a magnetic field inclination of about $45^{\circ}$. The second consists of a dynamic component interpreted as a flux tube with a lower magnetic field strength and more inclined (with respect to the vertical) than that of the background component. Moreover, the numerical code was developed to account for the different scenarios that arise from the picture extracted from the analysis of the observations. Apparently, we see the emergence of hot material and the subsequent submergence of the cooled off material, where the flows follow their own magnetic fields within the penumbra. For that purpose, the geometry of the problem was implemented for three scenarios: magnetic upflows, magnetic horizontal flows and magnetic downflows characterised by different inclination angles, tunable as input variables. Thus, the selection of observed single Stokes $I$ and $V$ profiles of the various spectral lines showing clear asymmetries was the next step. The main aim of the forward modelling procedure consisted then in the attempt of simulating such observed asymmetries. For that, a proper selection of the input parameters (magnetic field strength and inclination of both components, velocity for both components, temperature of the tube component in reference to the input background temperature, diameter of the tube, height of the tube in the atmosphere, filling factor, ...) following the existing models in the literature was introduced to the code to generate different models. The comparison of both, observed and synthetic profiles was performed by direct visualization. Thus, from systematic variations of the input parameters and comparison one acquires the capability of 'guessing' the atmospheric fine-structure and its dynamics.

We note in passing that the use of inversion techniques (del Toro Iniesta \& Ruiz Cobo 1996, Borrero 2004) is not advisable here. Firstly, inversion techniques require data with high signal-to-noise ratio and with high spectral resolution. Albeit of high spatial resolution, the data analysed here do not fulfill these needs. Low-noise, two-dimensional spectropolarimetric data with both high spectral and high spatial resolution will hopefully become available in the near future with new solar observing facilities. Secondly, inversion techniques cannot return more than the input picture (Borrero 2004). If one prescribes a one-component atmosphere one obtains results within the framework of a one-component atmosphere. If one asks for two components, the result will be given in terms of a two-component atmospehre, etc.

Some examples of our attempts of simulating the observed asymmetries by forward modelling are shown in Sec.5.3.1. From them, some general properties as the absence of cool upflows in penumbrae are inferred. After finding a large variety of asymmetries in 
the observed Stokes profiles and the difficulty to simulate all their characteristics at once, the main conclusions one arrives at are the following: On the one hand, with a spatial resolution better than 0.5 and moderate spectral resolution, one starts to resolve from the Stokes profiles, the effects of two or more components in the penumbral fine-structure, e.g. strong 'kinks' at different intensity levels in the Stokes $I$ profiles, which, so far, have not been presented elsewhere. On the other hand, two atmospheric component models are sufficient to explain some general properties of penumbrae, e.g. penumbral grains, but appear no to be sufficient (as it has been also claimed by other authors) to explain many of those asymmetries. A more complex and developed geometry, possibly from numerical magnetohydrodynamic simulations, should then be considered for numerical modelling. 


\section{Conclusions and outlook}

Spectropolarimetric observations from penumbrae in the Fe II $6149.3 \AA$ line and in the Fe I $6302 \AA$ line pair were analysed. By means of image reconstruction we achieved a spatial resolution of the spectroscopic data of 0.5 and better. The data in the two spectral regions were obtained from two different sunspots. Yet we may assume that the typical penumbral properties and dynamics are the same in more or less symmetric sunspots. The Fe II line is formed deeper in the atmosphere than the Fe I line pair and thus gives information about deep layers. The main results from the observations may be summarised as follows:

1. On scales larger than $00^{\prime \prime} 5$ the intensity pattern of penumbrae does not change when seen in radiation originating from either deep photospheric or higher layers $(300 \mathrm{~km})$. Yet at scales of $0^{\prime} .5$ and smaller the patterns in the two atmospheric ranges are clearly different. The structuring is height-dependent with vertical extents of the same scale as the horizontal thickness of the fibrils.

2. Interestingly, the small-scale channels of the Evershed flow coincide with different features on the limb and the disc-centre side of the sunspots. This can be understood in the picture of rising flux tubes of Schlichenmaier et al. (1998). It appears that at the limb side the flow is best seen in dark structures when the originally hot, rising gas has cooled down and the motion has turned horizontal or even downward. On the centre side, the flow is best seen in bright, rising structures. The asymmetry of the visibility of flows between centre and limb side was also seen in line bisectors by Schlichenmaier et al. (2004). This result from high-spatial-resolution observations confirms and specifies in more detail findings by Westendorp Plaza et al. (2001b). These authors also find, from lower resolution data, a visibility difference of velocities between limb and centre side and interprete this as an opacity effect.

3. Strong line-of-sight components of the magnetic fields are not found in bright filaments but in dark structures, somewhat displaced from the darkest parts, in both the limb and the centre side penumbrae. On the limb side, their positions do not coincide with those of the strongest velocity fields.

Although observations in Fe II $6149.3 \AA$ and in Fe I $6302 \AA$ were obtained from two different sunspots, the analysis of the two data sets gave a consistent picture. Generally, these findings confirm the uncombed magnetic field structure, i.e. the mixture of a magnetic more inclined and dynamical component embedded in a magnetic more vertical and almost static background atmosphere. They are also compatible with the picture of low lying magnetic flux tubes, which possibly even submerge back within the penumbra. 
This model was derived from low spatial resolution data, but with full polarimetric information by Bellot Rubio et al. (2003) assuming two-component atmospheres within the spatial resolution element.

The success of our analysis relies on both the two-dimensionality and the high spatial resolution of the spectropolarimetric information. Without two-dimensionality one would not see the differences between limb and centre side behaviour of intensity, velocity and magnetic fields and their relations. Also, it was possible only with sufficient spatial resolution to see in detail these relations and to detect the differences between limb and centre side.

Many aspects of penumbrae have remained inconclusive. One cannot define strict rules for relations between intensities from various atmospheric levels, velocities and magnetic fields as derived from the study on possible correlations between these magnitudes. There are special cases which apparently contradict the clear picture of Evershed flow channels with low lying magnetic fields. Also, high intensity in continuum or spectral line cores is not a matter of velocity or magnetic field, but primarily of temperature which itself also depends on the state of the ambient gas and on the history of the feature. Thus, bright does not necessarily mean weak (or strong) magnetic field.

An analysis of individual Stokes $I$ and $V$ profiles was performed by means of the synthesis of spectral lines by solving the one-dimensional radiative transfer problem. The modelling included small-scale magnetic flux tubes containing the Evershed flow embedded in a background atmosphere with little inclined magnetic fields. From this analysis, the following main conclusions can be drawn:

1. Cool upflows are definitely incompatible with the observations. On the contrary, the bright penumbral grains and their dynamics can be explained by hot upflows.

2. Few examples were shown for which the observed asymmetric spectral profiles could be reproduced, at least in part, by a two-component model.

3. The asymmetries shown by a large amount of the profiles are not fully reproducible. Thus, forward modelling of a two-component atmosphere means a first step to explain the observational results found on the penumbral fine-structure through numerical simulations.

The next attempt would be the development of a more complex geometry, possibly from numerical magnetohydrodynamic simulations.

Spectropolarimetric data on sunspot penumbrae with higher signal-to-noise ratio and with better spectral and spatial resolution than those presented here are already available thanks to the upgraded 'Göttingen' two-dimensional Fabry-Pérot spectrometer (Puschmann et al. 2006) in combination with the Kiepenheuer Adaptive Optics system (KAOS, von der Lühe et al. 2003) at the VTT. These data and the hopefully soon available, including measurements of the full Stokes vector, data from the new solar observing facilities 
are the next promising step for a more detailed study on the penumbral fine-structure, its magnetism and dynamics.

The picture of sunspot penumbrae that arise from our observations and the interpretation is partly compatible with the model of moving flux tubes by Schlichenmaier et al. (1998): In the penumbra, and possibly with the umbra near the penumbral border, magnetic flux tubes rise from sub-photospheric layers carrying hot material which moves outwards within the flux tubes at high speed, the Evershed flow. These are the bright penumbral grains, or bright heads of the penumbral filaments. Further outward, the flux tubes and the flow bend into a more horizontal direction while the material cools down. Within the penumbra, or at the outer penumbral border, the tubes and the flows submerge. the moving tube model also predicts that the tubes rise again, and submerge again, like a "sea serpent". This is still compatible with observation, yet certainly difficult to prove.

However, the structure and the dynamics of penumbrae appears substantially more complex. Our high-resolution polarimetric observations show that the penumbral filamentary structure depends on height. Filaments do exist at heights of $300 \mathrm{~km}$ and above, yet they have different locations and orientation than the low-lying filaments. And many spectral profiles from penumbral fine-structures require a more involved dynamical structure than the above two-component model which contains one flux tube in an unperturbed background.

The very recent penumbra model by Spruit \& Scharmer (2006) has not been considered in the simulation of the observed spectral profiles. This model, the "gappy penumbra", possesses field-free convective areas in sub-atmospheric layers along the radial direction with their cusps intruding into visible layers. The model is capable to account for the energy supply needed to balance the radiative losses from penumbrae. And possibly, the model explains the dark lanes ("cores") within long, bright filaments which were detected by Scharmer et al. (2002, and also seen in Fig.4.1). The dark cores would be produced by high opacity on top of the cusps and the bright filaments would be the hot walls of the convective layers. The model is static and, thus, does not deal with the Evershed flow. Besides, there is observational evidence that penumbral dynamical processes involve all atmospheric layers. Yet it will certainly be worthwhile to study the effects of such model structures on simulated broad-band intensities and Stokes profiles. 



\section{Bibliography}

Beck, C., Schlichenmaier, R., Collados, M., Bellot Rubio, L. R. \& Kentischer, T.: 2005, A\&A, 443, 1047

Beckers, J. M.: 1968, Sol. Phys., 3, 258

Beckers, J. M. \& Schröter, E. H.: 1969, Sol. Phys., 10, 384

Bello González, N., Okunev, O. V., Domínguez Cerdeña, I., Kneer., F. \& Puschmann, K. G.: 2005, A\&A, 434, 317

Bello González, N., Okunev, O. V. \& Kneer, F.: 2005 in 11th European Solar Physics Meeting "The Dynamic Sun: Challenges for Theory and Observations", eds. D. Danesy, S. Poedts, A. De Groof \& J. Andries, ESA SP-600, 56

Bellot Rubio, L. R.: 2004, in Reviews in Modern Astronomy: The Sun and Planetary Systems - Paradigms for the Universe, Wiley-VCH, 17, 21

Bellot Rubio, L. R., Balthasar, H., Collados, M. \& Schlichenmaier, R.: 2003, A\&A, 403, 47

Bellot Rubio, L. R., Balthasar, H. \& Collados, M.: 2004, A\&A, 427, 319

Bendlin, C. \& Volkmer, R.: 1995, A\&AS, 112, 371

Bendlin, C., Volkmer, R. \& Kneer, F.: 1992, A\&A, 257, 817

Biermann, L.: 1941, Vierteljahresschr. Astr. Ges., 76, 194

Börner, P. \& Kneer, F.: 1992, A\&A, 259, 307

Born, M. \& Wolf, E.: Principles of Optics, Cambridge University Press, 7th edition, 1999

Borrero, J. M.: 2004, PhD thesis, Göttingen University

Borrero, J. M., Solanki, S. K., Bellot Rubio, L. R., Lagg, A. \& Mathew, S. K.: 2004, A\&A, 422, 1093

Bray R.J. \& Loughhead R.E.: 1964, Sunspots, Chapman and Hall

Charbonneau, P.: 2005, Living Reviews in Solar Physics, Max Planck Institute for Solar System Research, 2, 2

Collados, M.: 1999, in Third Advances in Solar Physics Euroconference: Magnetic Fields and Oscillations, eds. B. Schmieder, A. Hofmann \& J. Staude, ASP Conf. Ser. 184, 3 
de Boer, C. R.: 1993, PhD thesis, Göttingen University

Degenhardt, D. \& Wiehr, E.: 1994, A\&A, 287, 620

del Toro Iniesta, J. C.: 2003, Introduction to Spectropolarimetry, Cambridge University Press

del Toro Iniesta, J. C. \& Ruiz Cobo, B.: 1996, Sol. Phys., 164, 169

del Toro Iniesta, J. C., Tarbell, T. D. \& Ruiz Cobo, B.: 1994, ApJ, 436, 400

Denker, C.: 1996, PhD thesis, Göttingen University

Denker, C.: 1998, Sol. Phys., 180, 81

Eibe, M. T., Mein, P., Roudier, Th. \& Faurobert M.: 2001, A\&A, 371, 1128

Evershed, J.: 1909, MNRAS, 69, 454

Fried, D. L.: 1966, J. Opt. Soc. America, 56, 1372

Gingerich, O., Noyes, R. W., Kalkofen, W. \& Cuny, Y.: 1971, Sol. Phys., 18, 347

Gokhale, M. H. \& Zwaan, C.: 1972, Sol. Phys., 26, 52

Giovanelli, R. G. \& Jones, H. P.: 1982, Sol. Phys., 79, 267

Grigorjev, V. M. \& Katz, J. M.: 1972, Sol. Phys., 22, 199

Hale, G. E.: 1908, ApJ, 28, 315

Hirzberger, J. \& Kneer, F.: 2001, A\&A, 378, 1078

Hofmann, A.: 2000, private communication

Jahn, K.: 1989, A\&A, 222, 264

Jahn, K.: 1997, in First Advances in Solar Physics Euroconference: Advances in Physics of Sunspots, eds. B. Schmieder, J. C. del Toro Iniesta \& M. Vázquez, ASP Conf. Ser. 118,122

Janssen, K.: 2003, PhD thesis, Göttingen University

Keller, C. U. \& von der Lühe, O.: 1992, A\&A, 261, 321

Kinman, T. D.: 1952, MNRAS, 112, 425

Korff, D.: 1973, J. Opt. Soc. Am., 63, 971

Koschinsky, M.: 2001, PhD thesis, Göttingen University

Koschinsky, M., Kneer, F. \& Hirzberger, J.: 2001, A\&A, 365, 588

Krieg, J., Wunnenberg, M., Kneer, F., Koschinsky, M. \& Ritter, C.: 1999, A\&A, 343, 983 Landi Degl'Innocenti, E.: 1992, in Solar Observations: Techniques and interpretations, 
eds. F. Sánchez, M. Collados \& M. Vázquez, Cambridge University Press, 71

Labeyrie, A.: 1970, A\&A, 6, 85

Lindsey, C. \& Braun, D. C.: 1998, ApJ, 509, 129

Lites, B. W., Scharmer, G. B. \& Skumanich, A.: 1990, ApJ, 355, 329

Lites, B. W., Elmore, D. F., Seagraves, P. \& Skumanich, A.: 1993, ApJ, 418, 928

Livingston, W.: 2002, Sol. Phys., 207, 41

Maltby, P.: 1964, Astrophys. Norvegica, 8, 205

Martínez Pillet, V.: 1997, in First Advances in Solar Physics Euroconference: Advances in Physics of Sunspots, eds. B. Schmieder, J. C. del Toro Iniesta \& M. Vázquez, ASP Conf. Ser. 118, 212

Martínez Pillet, V.: 2000, A\&A, 361, 734

Mein, P.: 1971, Sol. Phys., 20, 3

Meyer, F. \& Schmidt, H. U.: 1968, Z. Ang. Math. Mech., 48, 218

Montesinos, B. \& Thomas, J. H.: 1993, ApJ, 402, 314

Montesinos, B. \& Thomas, J. H.: 1997, Nature, 390, 485

Moore R. L. \& Rabin, D.: 1985, Annual Reviews Astron. Astroph., 23, 239

Müller, D. A. N., Schlichenmaier, R., Steiner, O. \& Stix, M.: 2002, A\&A, 393, 305

Neckel, H.: 1999, Sol. Phys., 184, 421

Okunev, O.: 2004, PhD thesis, Göttingen University

Pérez Rodríguez, E. \& Kneer, F.: 2002, A\&A, 395, 279

Puschmann, K. G., Kneer, F., Seelemann, T. \& Wittmann, A. D.: 2006, A\&A, 451, 1151

Rees, D. E. \& Semel, M. D.: 1979, A\&A, 74, 1

Rees, D. E., Murphy, G. A. \& Durrant, C. J.: 1989, ApJ, 339, 1093

Rimmele, T. R.: 1995, A\&A, 298, 260

Rouppe van der Voort, L. H. M.: 2002, A\&A, 389, 1020

Rouppe van der Voort, L. H. M., Lödfahl, M. G., Kiselman, D. \& Scharmer, G. B.: 2004, A\&A, 414, 717

Sánchez Almeida, J.: 2005, ApJ, 622, 1292

Sánchez Almeida, J. \& Lites, B. W.: 1992, ApJ, 398, 359

Scharmer, G. B., Gudiksen, B. V., Kiselman, D., Löfdahl, M. G. \& Rouppe van der Voort, 
L. H. M.: 2002, Nature, 420, 151

Schlichenmaier, R.: 2003, in Current Theoretical Models and Future High Resolution Observations, eds. A. A. Pevtsov, \& H. Uitenbroek, ASP Conf. Ser. 286, 211

Schlichenmaier, R. \& Collados, M.: 2002, A\&A, 381, 668

Schlichenmaier, R. \& Schmidt, W.: 2000, A\&A, 358, 1122

Schlichenmaier, R., Bellot Rubio, L. R. \& Tritschler, A.: 2004, A\&A , 415, 731

Schlichenmaier, R., Jahn, K. \& Schmidt, H. U.: 1998, A\&A, 337, 897

Schlichenmaier, R., Müller, D. A. N., Steiner, O. \& Stix, M.: 2002, A\&A, 381, 77

Schmidt, W.: 2003, Astron. Nachr., 324, 374

Schröter, E. H.: 1965, ZAp, 41, 141

Schüssler, M. \& Rempel, M.: 2005, A\&A, 441, 337

Semel, M.: 1967, Annales d'Astrophysique, 30, 513

Sobotka, M. \& Sütterlin, P.: 2001, A\&A, 380, 714

Sobotka, M., Brandt, P. N. \& Simon, G. W.: 1999, A\&A, 348, 621

Socas Navarro, H.: 1999, PhD thesis, La Laguna University

Solanki, S. K.: 2003, A\&A Review, 11, 153

Solanki, S. K., \& Montavon, C. A. P.: 1993, A\&A, 275, 283

Solanki, S. K., Rüedi, I. \& Livingston, W.: 1992, A\&A, 263, 339

Spruit, H. C. \& Scharmer, G. B.: 2006, A\&A, 447, 343

Stanchfield, D. C. H., Thomas, J. H. \& Lites, B. W.: 1997, ApJ, 477, 485

Stellmacher, G. \& Wiehr, E.: 1971, Sol. Phys., 17, 21

Sütterlin P.: 2001, A\&A, 374, L21

Sütterlin, P., Bellot Rubio, L. R. \& Schlichenmaier, R.: 2004, A\&A, 424, 1049

Title, A. M., Frank, Z. A., Shine, R. A., Tarbell, T. D., Topka, K. P., Scharmer, G. B. \& Schmidt W.: 1993, ApJ, 403, 780

Tritschler, A., Schlichenmaier, R., Bellot Rubio, L. R. and the KAOS Team (Soltau, D., Berkefeld, T., Schelenz., T.): 2004, A\&A, 415, 717

Unsöld, A: 1955, Physik der Sternatmosphären, 2nd edition, Springer-Verlag, 333

Vela Villahoz, E., Sánchez Almeida, J. \& Wittmann, A. D.: 1994, A\&A, 103, 293

Vernazza, J. E., Avrett, E. H. \& Loeser, R.: 1981, A\&A, 45, 635 
von der Lühe, O.: 1984, J. Opt. Soc. Am., 1, 510

von der Lühe, O., Soltau, D., Berkefeld, T. \& Schelenz, T.: 2003, SPIE, 4853, 187

Weigelt, G. P.: 1977, Optics Comm., 21, 55

Weiss, N. O.: 1997, in Advances in the Physics of Sunspots, eds. B. Schmieder, J. C. del Toro Iniesta \& M. Vázquez, ASP Conf. Ser. 118, 21

Weiss, N. O.: 2002, Astron. Nachr., 323, 371

Westendorp Plaza, C., del Toro Iniesta, J. C., Ruiz Cobo, B., Martínez Pillet, V., Lites, B. \& Skumanich, A.: 2001, ApJ, 547, 1130

Westendorp Plaza, C., del Toro Iniesta, J. C., Ruiz Cobo, B. \& Martínez Pillet, V.: 2001, ApJ, 547, 1148

Wiehr, E.: 1999, in Third Advances in Solar Physics Euroconference: Magnetic Fields and Oscillations, eds. B. Schmieder, A. Hofmann, \& J. Staude, ASP Conf. Ser. 184, 86

Wiehr, E. \& Degenhardt, D.: 1992, A\&A, 259, 313

Wiehr, E. \& Stellmacher, G.: 1989, A\&A, 225, 528

Wiehr, E., Koch, A., Knölker, M., Küveler, G. \& Stellmacher, G.: 1984, A\&A, 140, 352

Wilson, A.: 1774, Philosophical Transactions, 64, 6

Wittmann, A. D.: 1971, Sol. Phys., 20, 365

Wittman, A. D. \& Xu, Z. T.: 1987, A\&AS, 70, 83

Zwaan, C.: 1978, Sol. Phys., 60, 213 



\section{Acknowledgements}

These years of Thesis work in Göttingen have been a crucial part of my postgraduate formation and personal life. I want to thank Franz Kneer who contributed not only with his vast knowledge and interest but also with his constant encouragement and motivation.

This work has been carried out in the Georg-August-Univarsität Göttingen, firstly at the Universitäts-Sternwarte Göttingen and latter at the Astrophysikalisches Institut Göttingen (AIG). In both the nice environment and the hospitality of all my colleagues formed an ideal working atmosphere and has made me feel at home. I want to thank my friends and colleagues with who I have shared intense hours of common work and laughs: thanks Oleg, Klaus, Markus, Ita, Julián, Bruno, Miguel, Julio, Arabela, Moritz,...

I gratefully acknowledge a fellowship of the Graduiertenkolleg GRK 140 Strömungsinstabilitäten und Turbulenz by the Deutsche Forschungsgemeinschaft (DFG) as well as the financial support by the International Max Planck Research School (IMPRS) On Physical Processes in the Solar System and Beyond. An important part of the project were the observations with the German Vacuum Tower Telescope operated by the KiepenheuerInstitut für Sonnenphysik, Freiburg, at the Observatorio del Teide. I want to thank Itahiza Domínguez for providing me with part of the data I worked with and to David Orozco for useful comments on the radiative transfer code. I would like to especially thank Dr. D. Schmitt, for its support and continuous concern with us, the students of the IMPRS. Thanks also to Dr. R. Schlichenmaier, Dr. H. Balthasar and Dr. W. Wiehr for letting me participate in their observational campaign on 2004 and to Dr. A. Wittman for interesting talks and complete references.

I want to make a especial mention to Niina, Dana \& Josh, Jan, Stano, Etienne, Mohsen, Vasily and Juan, Vanina \& Lucía, all nice friends I have met in this period et "bouchou trouz" à tous mes amis bretons!.

Por último quiero agradecer a papi, a mami y a Tavo el apoyo infinito que he recibido de ellos en esta mi aventura internacional. Con ellos, mi mayor tesoro, su dedicación y su ánimo constante las distancias se han acortado y mi trabajo ha sido una meta común. Este agradecimiento es extensible a toda mi familia, incluyendo a los que se fueron y a los que han llegado con alegría nueva. Y, por supuesto, a mis incondicionales, Ré, Antonia, Ada, Carmen, Sarah, Ila, Siby, Alex, чико у todos aquellos con quienes compartí esta andadura. Sólo me quedan unas últimas palabras para un amigo: igracias Franz por tu simpatía!, gracias Don Quijote por mostrarme un lugar de "la mancha" de cuyo nombre no quiero acordarme... 



\section{Publications}

Bello González, N., Okunev, O. V., \& Kneer., F.: Synthesis of Stokes profiles from a two component penumbral model. Proceedings of the 11th European Solar Physics Meeting "The Dynamic Sun: Challenges for Theory and Observations" (ESA SP-600). 11-16 September 2005, Leuven, Belgium. Editors: D. Danesy, S. Poedts, A. De Groof and J. Andries. Published on CDROM., p.56.1

Valdivielso Casas, L., Bello González, N., Puschmann, K. G., Sánchez-Andrade Nuño, B. \& Kneer, F.: Analysis of polarimetric sunspot data from TESOS, Proceedings of the International Scientific Conference on Chromospheric and Coronal Magnetic Fields (ESA SP-596). 30 August - 2 September 2005, Katlenburg-Lindau, Germany. Editors: D.E. Innes, A. Lagg \& S.K. Solanki, Published on CDROM, p.67.1

Bello González, N., Okunev, O. V. \& Kneer., F.: Study of Asymmetries of Stokes profiles from high spatial resolution spectropolarimetry, Proceedings of the International Scientific Conference on Chromospheric and Coronal Magnetic Fields (ESA SP-596). 30 August - 2 September 2005, Katlenburg-Lindau, Germany. Editors: D.E. Innes, A. Lagg \& S.K. Solanki, Published on CDROM, p.50.1

Bello González, N., Okunev, O. V., Domínguez Cerdeña, I., Kneer., F. \& Puschmann, K. G., Polarimetry of sunspot penumbrae with high spatial resolution 2005, A\&A, vol. 434,317

Bello González, N., Okunev, O. V., \& Kneer., F.: Spectropolarimetry in a sunspot penumbra at high spatial resolution, "Solar magnetic phenomena", (Kärnten, Austria 2003), 3rd Summerschool and Workshop held at the Solar Observatory Kanzelhöhe. Eds.: A. Hanslmeier, A. Veronig, and M. Messerotti. Astronomy and Astrophysics Space Science Library, vol. 320, Springer, Dordrecht, The Netherlands, 2005., p.183-186

Bello González, N., Okunev, O. V., Domínguez Cerdeña, I. \& Kneer., F.: Polarimetry in sunspot penumbrae at high spatial resolution, Astronomische Nachtrichten, Vol. 325, Supplement 1, Short Contributions Presented at the Annual Scientific Meeting of the Astronomische Gesellschaft and the Czech Astronomical Society in Prague, September 20-25, 2004., p.79

Bello González, N. \& Kneer., F.: Velocity and Magnetic Fields in Sunspot Penumbrae at Hight Spatial and Spectral Resolution Astronomische Nachrichten, Supplementary Issue 3, Vol. 324, Short Contributions of the Annual Scientific Meeting of the Astronomische Gesellschaft in Freiburg, September 15-20, 2003, p.28 



\title{
Lebenslauf
}

\author{
Name: $\quad$ Nazaret Bello González \\ Geburtsdatum: $\quad 05.07 .1977$ \\ Geburtsort: Granadilla de Abona (Santa Cruz de Tenerife), Spanien \\ Familienstand: $\quad$ ledig \\ Eltern: Antonio Bello Rodríguez \\ María Dolores González del Pino \\ Staatsangehörigkeit: Spanisch
}

Schulbildung: Oktober 1983 - Juni 1991 Grundschule am Colegio

"Almirante Carrero Blanco"

(Granadilla de Abona, Sta. Cruz de Tenerife)

Oktober 1991 - Juni 1995 Weiter führende Schule am "I. B.

Adelantado Fernández de Lugo"

(Granadilla de Abona, Sta. Cruz de Tenerife)

Studium: $\quad$ September 1995 - Juni 2002 Physikalische Fakultät

in der Universität La Laguna

$2001-2002$

Studiums-Auslandsjahr im Rahmen

des internationalen Austauschprogramms

"ERASMUS" an der Universitäts-Sternwarte

Göttingen mit Abschlussarbeit:

Estudio del Stokes $V$ en Fe II 6149.3 :

Campo Magnético en Penumbra

Promotion: $\quad$ Februar 2003 - May 2006

Promotion an der Universitäts-Sternwarte

Göttingen (seit Juni 2005 Institut für Astrophysik Göttingen, IAG)

Stipendium des Graduiertenkolleg GRK 140, Stipendium der International Max Planck

Research School (IMPRS) 